-

W.1.

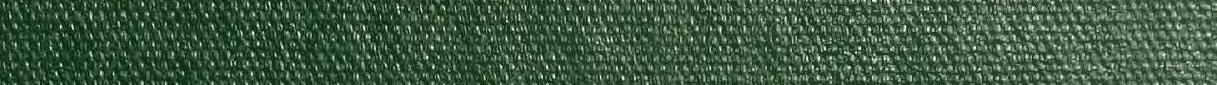

Q

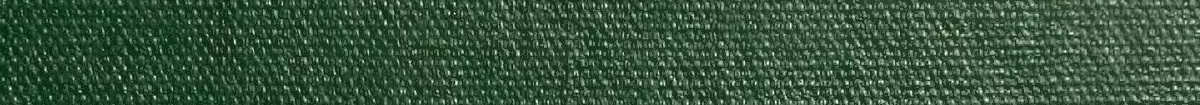

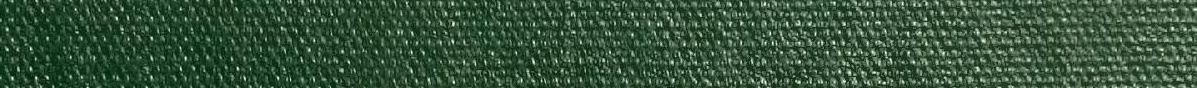

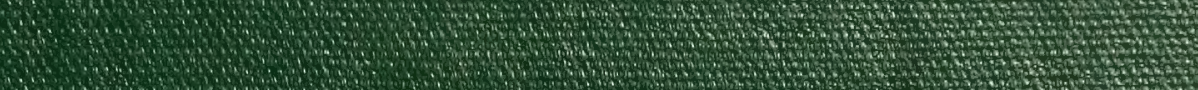

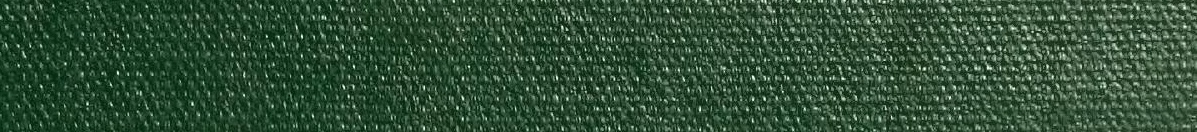

(1) \begin{tabular}{|c|c|c|c|}
\hline \\
4
\end{tabular} (3) (1)

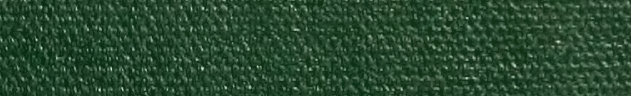

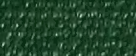
(5)

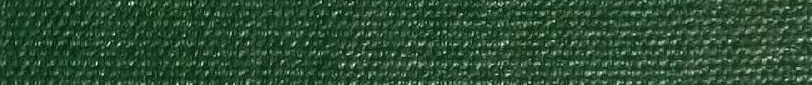

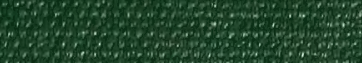

W.1. \begin{tabular}{|c|c|c|}
\hline \\
3
\end{tabular} H.

C.7.

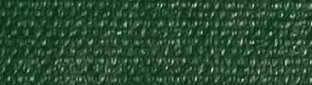

1. $7 x^{2}$

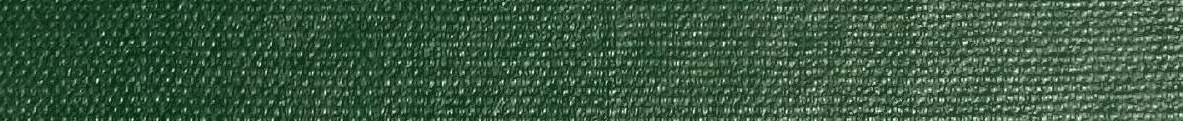

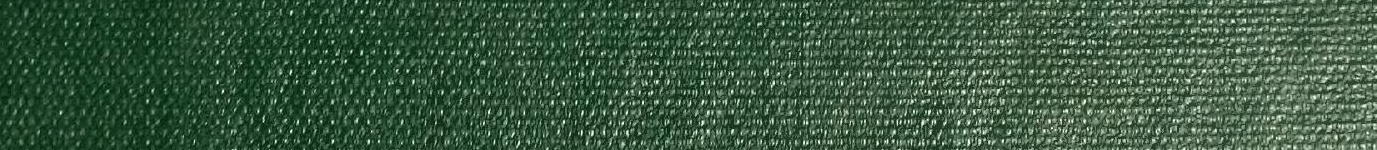
13.

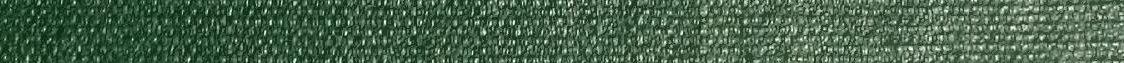

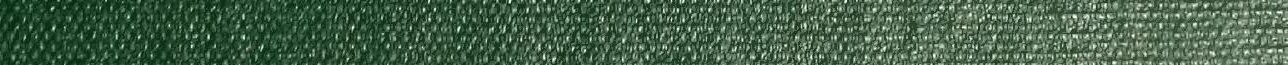

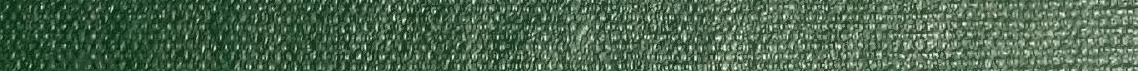




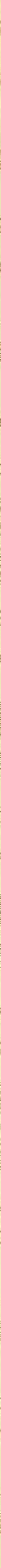

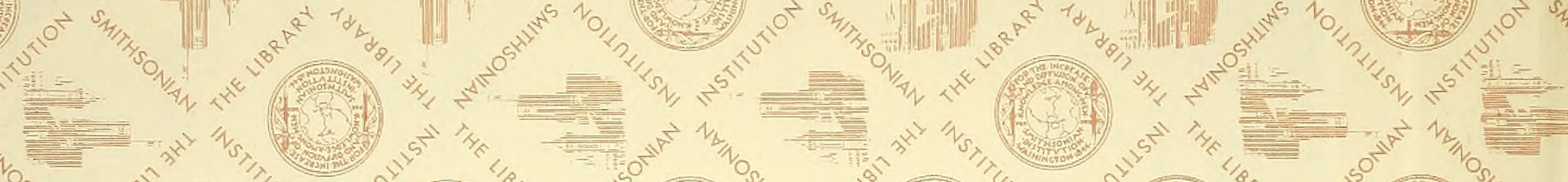
2)

韾 tos

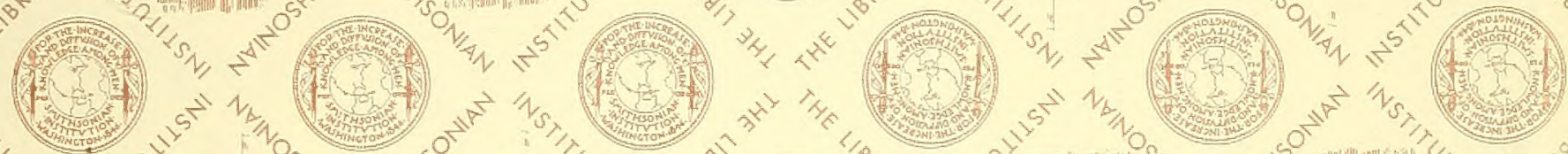

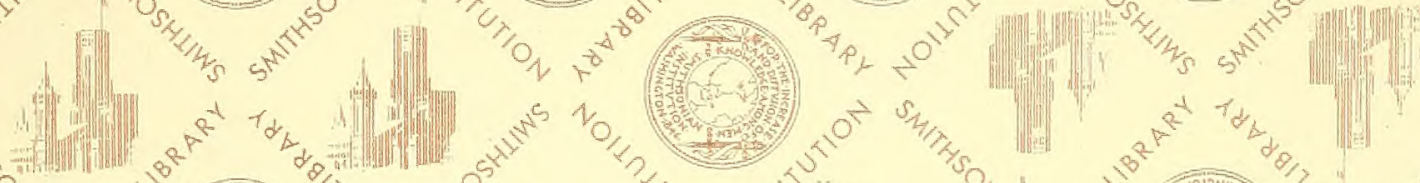
(19i)
(3) 

. 



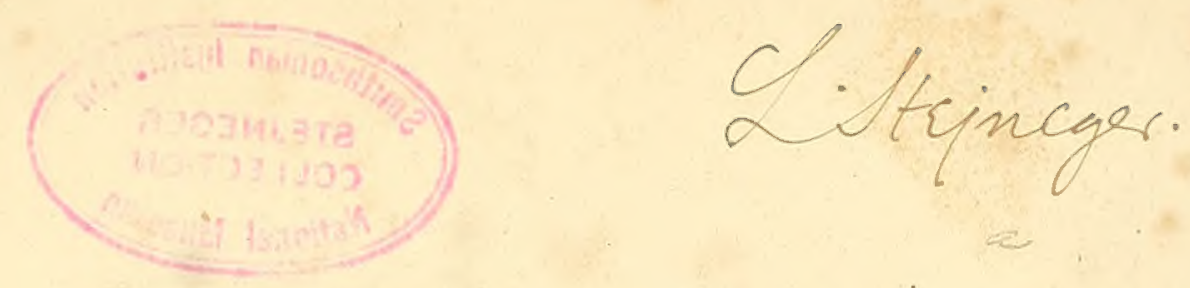

\title{
DESCRIPTION
}

\section{DES REPTILES}

NOUVEAUX OU IMPARFAITEMENT CONNUS

DE LA COLLECTION DU MUSÉUM D'HISTOIBE NATURELLE

ET

\section{REMARQUES SUR LA CLASSIFICATION}

ET LES GARAGTÈRES DES REPTILES.

\author{
PREMIER MÉMOIRE \\ ORDRE DES CHÉLONIENS ET PREMIÈres FAMILLes DE L'ORDRE DES SAURIENS \\ (CROCODILIENS ET CAMÉLEONIENS) \\ PAR LE DOCTEUR AUGUSTE DUMÉRIL \\ AIDE-NATURALISTE AU MUSÉUM,
PROFESSEUR-AGRÉGE A LA PAGULTÉ DE MÉDECINE, \\ MEMBRE DE LA SOCIÉTÉ PHILOMATHIRUE.
}

Le travail dont je commence aujourd'hui la publication a pour but de faire connaitre les acquisitions nouvelles qui ont enrichi la Collection des Reptiles, depuis la publication des six premiers volumes et du huitième de l'Erpétologie générale.

Le bilan exact de la science avait été dressé dans cet ouvrage, pour la rédaction duquel mon père, aidé par l'habile et savante collaboration de G. Bibron, avait dù passer en revue les matériaux imArchives du Múséusi. Tome VI. 
menses, et si précieux, rassemblés de longue date, et à grands frais, au Muséum d'histoire naturelle. De cette étude en commun où l'intelligence si droite et le tact naturel et si sûr de Bibron s'appuyaient sur la vaste expérience et sur les habitudes d'analyse méthodique de mon père, il est sorti un Traité didactique de l'histoire naturelle des Reptiles, dont la valeur et l'utilité sont si universellement admises, qu'il est devenu le guide désormais indispensable à quiconque veut étudier cette branche si étendue de la Zoologie. En m'exprimant ainsi sur une ceuvre due en partie à mon père, je n'ai pas la crainte de voir contredit le jugement que je me permets d'émettre avec une entière liberté d'esprit, et en ne me faisant que l'écho des appréciations dont chacun des volumes a été l'objet, au moment où il a paru. Je saisis, d'ailleurs, avec empressement, cette occasion qui m'est offerte, de payer au naturaliste si prématurément emporté au milieu de ses utiles travaux, un faible tribut de reconnaissance pour les précieux services qu'il a rendus à la science, et de mêler ma voix amie aux voix éloquentes et plus dignes que la mienne, mais non moins sincères, qui ont, à diverses reprises, rendü justice à sa mémoire.

Investi, par l'honorable confiance de MM. les professeurs, des fonctions que Bibron remplissait avec tant de succès, j’ai été appelé à soumettre à un nouvel examen toute la Collection erpétologique, et c'est pendant la durée de ce travail, que j’ai pu apprécier, mieux que je ne l'avais encore fait, l'utile secours que l'Erpélologie générale fournit pour la détermination exacte des genres et des espèces. Un défaut cependant peut y être reconnu, mais ce défaut est l'cuvre du temps et non des auteurs. Il réside dans les lacunes qu'y ont fait naître forcément, et en un petit nombre d'années, les accroissements continuels des Collections zoologiques du Muséum.

Douze ans, en effet, ont suffi pour rendre incomplet ce vaste Catalogue méthodique et descriptif.

Un complément lui était donc devenu indispensable, et ce sont ces vides que je me suis efforcé de combler dans le Catalogue beau- 
coup plus abrégé qui se publie, en ce moment, par mes soins, et sous la direction de mon père.

Des espèces et mème des genres ont été indiqués aux naturalistes, postérieurement à la publication des volumes de l'Erpétologie générale, et l'on a fait entrer dans le classement général adopté dans cet ouvrage des animaux inconnus jusqu'alors et qui, par leurs caractères, n’appartenaient à aucune des coupes établies par la science. Il fallait donc faire connaitre ces espèces et ces genres quand ils sont représentés au Muséum par un ou plusieurs échantillons.

Un autre motif d'intérèt s'attachait à la révision générale des Collections, car un assez grand nombre de Reptiles que le Musée de Paris possède seul, sans doute, puisqu'ils n'avaient encore été décrits nulle part, restaient inconnus. Leur détermination et leur description devaient nécessairement prendre place dans ce nouveau travail. En raison de sa destination, d'ailleurs, puisqu'il constitue un véritable inventaire raisonné des Collections du Musée de Paris, il ne mentionne point certaines espèces, indiquées comme nouvelles par les auteurs, quand ce Musée ne les possède pas.

La forme concise qu'il est convenable de donner à un Catalogue m'a tenu dans certaines limites qu'il n'aurait peut-ètre pas été sans quelque intérêt de pouvoị franchir, afin de faire ressortir les particularités les plus saillantes de l'histoire de ces animaux nouveaux, et surtout afin de présenter quelques considérations générales sur les familles et sur les genres ainsi augmentés par l'addition d'espèces inédites.

C'est pour obvier à ces inconvénients, et pour compléter de cette facon, ainsi que par l'adjonction d'un certain nombre de planches, le travail primitif, que j'ai obtenu de la bienveillance de MM. les Professeurs l'autorisation de faire insérer dans les Archives du Muséum ce Complément, ou plutôt cette sorte de Commentaire du Catalogue méthodique de la Collection des Reptiles. 


\section{ORDRE DES TORTUES OU CHÉLONIENS.}

Cet ordre si naturel est parfaitement caractérisé par cette courte diagnose: Corps protégé par une carapace et par un plastron; quatre pattes; pas de dents. En raison des différences remarquables dans le genre de vie des animaux qu'il comprend, il a été facile de le subdiviser en quatre groupes dont les dénominations sont tirées du milieu dans lequel vivent les espèces, selon qu'elles sont terrestres, ou qu'elles recherchent les eaux peu courantes des marais ou des petites rivières, ou qu'elles habitent soit les eaux vives des grands fleuves, soit la mer.

On a rapporté à cet ordre un assez grand nombre d'espèces nouvelles depuis l'année 1835, où parut le deuxième volume de l'Histoire naturelle générale des Reptiles, consacré, presque tout entier, à son étude. On en a la preuve par le tableau suivant:

Nombre d'espèces de Chéloniens.

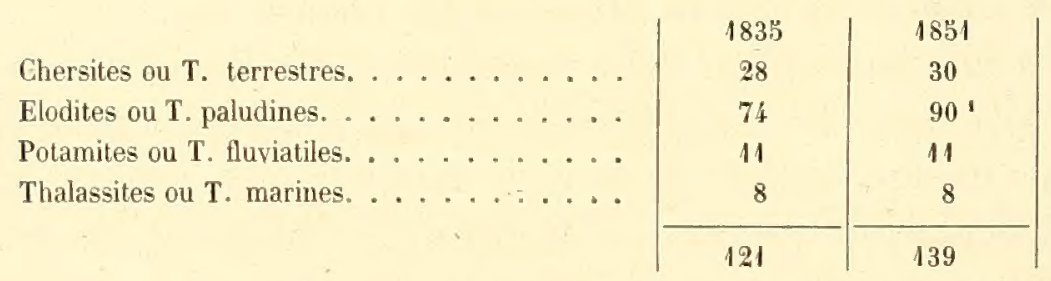

\section{TORTUES TERRESTRES OU CHERSITES.}

Deux Chersites nouvelles dont le genre de vie terrestre est parfaitement indiqué par leurs pattes courtes, informes, tronquées, à doigts réunis en moignon arrondi, et par leur carupace très-bombée (caractères essentiels de cette famille), doivent être décrites : elles appartiennent au genre тоRтuE. Elles en présentent, en effet, les caractères distinctifs, importants, tirés de la présence de cinq ongles en avant, et de quatre en arrière, puis de l'immobilité des pièces qui composent la carapace; celles du plastron sont soudées

1. Le Catalogue en indique 88 seulement, mais il faut ajouter à ce nombre une Podocnémide de l'Amérique du Sud (P. DE LÉwT) tout récemment reçue par le Muséum, et l'ÉM. DE L'OnÉGon, Holbr. 
entre elles. C'est dans ce grand genre, au reste, qu'il faut ranger la plupart des Tortues de terre, puisqu'il en comprend vingt-quatre sur trente.

Dans le genre Howopode, au contraire, ou la carapace, ainsi que le plastron, sont d'une seule pièce, comme dans le précédent, mais où les ongles sont au nombre de quatre seulement aux membres antérieurs, de même qu'aux postérieurs, il n'y a que deux espèces.

Quant au petit genre Prxıs, à plastron mobile en avant, il a été établi pour une espèce unique, et l'on n'en compte pas plus de trois dans le genre Cinixys, si remarquable par la mobilité de l'extrémité postérieure de la boîte osseuse.

La première des deux Tortues nouvelles est très-probablement originaire de l'Afrique australe, car elle est entièrement conforme à celle que M. Smith a décrite et figurée dans la $6^{\circ}$ planche $(R e p t$.) de son bel ouvrage (Zool. of the South Afr.), et qu'il nomme T. M-Dentelée, T. semi-serrata. Elle peut être très-facilement distinguée de ses congénères par la diagnose suivante :

GENHE TOHTUE. TESTUEO. BRONGNIART,

\section{I. - 4 bis ${ }^{\bar{T}}$ Tortue Mi-dentelée, $T$. semi-serrala, A Sinith.}

A. Smith (Illustr. of the zoul. of South. Afr. pl.6, texte sans pagination.)

The toothed Cap Tortoise, T. semi-serrata, Gray, Cat. of Tort., p. 8.

Tortue M-Dextelée, $T$. semi-serrata, Dum. et A. Dum. Catal. des Rept., p. $3, n^{\circ} 4$ bis.

Plaques antérieures et postérieures du limbe à pointe aigué, relevée et tournée en dehors; plaque nuchale en forme de triangle à sommel antérieur; sus-caudale simple, verticalement dirigée en bas; toutes les pièces de la carapace fortement striées, à aréole médiane un peu enfoncée, et couverte de fines granulations; pattes de devant armées à leurs faces antérieure et externe de grandes écailles tuberculeuses, épaisses et pointues; un fort tubercule conique à la face interne du talon.

A ces caractères essentiels, il faut en joindre d'autres, propres à mieux faire connaître cette jolie espèce qui, par la petitesse de sa taille, par ses

I. Dans le Catal., pour la numération des espèces dont la description ne se trouve pas dans l'Erpét. génér., j'indique par les mots bis, ter et même quater, placés à la suite du numéro que porte, dans ce dernier ouvrage, l'espèce auprès de laquelle la nouvelle vient se ranger, la place naturelle que celle-ci doit occuper dans la classification. J'évite ainsi. de troubler la série des numéros primitivernent adoptés. Je les répète ici, mais chacun d'eux est précédé d'un chiffre romain qui devient un numéro d'ordre spécial, joint à chacune des descriptions contenues dans ce premier Mémoire. 
couleurs, et par tout l'ensemble de sa conformation, offre une ressemblance assez marquée avec la Tontue actinode, et surtout avec la Tortue géoméTRıUe, dont il est cependant facile de l'éloigner.

La carapace est ovale, un peu allongée, légèrement convexe en dessus, mais bien moins relevée en dos d'âne que chez la seconde de ces deux Tortues à laquelle nous avons à la comparer. Toutes les plaques, d'ailleurs, sont moins bombées que chez cette dernière, et les dentelures profondes et acérées des bords antérieur et postérieur de la carapace ne permettent aucune confusion avec les espèces les plus voisines, dont le limbe est à peine échancré dans les mêmes régions.

Par la forme en triangle de sa plaque nuchale, elle s'éloigne de la T. GÉoMétrique où cette plaque est linéaire; et chez les T. actinode et Panthìre, il y a, dans le point correspondant, une échancrure, par suite de l'absence de cette pièce du limbe. Plus fortement protégée que ses congénères par l'armure écailleuse de ses pattes antérieures, elle porte, en outre, un tubercule conique, de chaque còté de la quene.

Par les stries nombreuses et profondes de ses écailles, et par la disposition radiée du jaune-brunâtre, dont elles sont couvertes dans les points où manque la teinte noire du fond, elle présente une analogie remarquable avec les $\mathrm{T}$. actinode et Géométrigde, très-semblables entre elles par leur système de coloration. La nuance plus foncée des lignes jaunes ou plutôt presque brunes, et leur largeur beaucoup plus considérable, sont cependant ici d'excellents moyens de distinction. En s'en tenant à ce caractère, que du centre aréolaire de chaque écaille, il part de larges lignes d'un jaune-brun qui, coupant, sous forme de bandes, la couleur du fond, vont rejoindre les lignes des plaques voisines, on sait ce qu'il importe le plus de connaitre relativement à ce système de coloration que M. Smith a décrit en détail, et que le dessin qu'il en a donné représente avec exactitude.

Ce naturaliste en a examiné un grand nombre d'exemplaires, et il n'en a jamais vu qui dépassât en longueur 5 pouces anglais : c'est à peu près la taille de l'échantillon unique du Musée de Paris, puisque sa carapace porte en longneur $o^{m}$ og 8 senlement; le plastron a $o^{m}$ o84. La largeur du disque est, au niveau de la région brachiale, de $\mathrm{o}^{\mathrm{m}} \mathrm{o} 6 \mathrm{o}$, et au niveau de la région fémorale, de $0^{\mathrm{m}} 067$. La tète est longue de $\mathrm{o}^{\mathrm{m}} 025$ et large de $0^{\mathrm{m}}$ o 18 ; la longueur de la queue est de $0^{\mathrm{m}}$ o: 5 . 
La T. GÉor., comme celle-ci, reste petite, car on ne la voit pas dépasser en longueur $\mathrm{o}^{\mathrm{W}} 20$, ce qui est cependant déjà beaucoup plus.

La dernière analogie, enfin, se tire de la communauté d'origine; mais la T. Mi-DENTELÉE se trouve exclusivement dans les régions les plus méridionales du continent Africain, tandis que l'autre vit, en outre, à Madagascar.

M. Smith signale encore une autre Tortue : c'est la T'est. Verreauxii, assez semblable aux deux espèces qui viennent déjà de servir de termes de comparaison, comme le montrent la pl. 8 de (Zool. of the S. Afr.), et la description annexée.

Les caractères principaux de cette espèce que M. Gray (Cat. of 'Tort., p. 9) suppose n'ètre qu'une variété de la $T$. géométrique, consistent dans la dìrection du limbe qui, par sa projection en dehors, forme, à son bord interne, un angle rentrant, assez profond, dans le point où ses plaques s'articulent avec les costales, et dans la forme de la nuchale qui porte un sillon médian dans le sens de sa longueur, et des stries transversales.

Le Muséum ne possède pas cette espèce.

La seconde espèce du genre Tortue mentionnée sur le Catalogue, et dont la description originale ne remonte pas au delà de l'année 1844 , a été nommée Tesiudo Emys par M. Salomon Müller, dans le beau et précieux Traité d'Histoire naturelle des possessions Néerlandaises d'outre-mer, dû aux membres de la Commission des sciences naturelles aux Indes-Orientales ${ }^{\mathrm{I}}$.

Cette dénomination, destinée à rappeler qu'il existe quelque analogie entre l'animal auquel elle a été donnée, et les représentants du genre Émyde, ne pouvait être conservée. Les règles de la nomenclature exigent qu'un nom de genre ne serve pas pour une simple désignation spécifique; aussi est-il remplacé dans le Catal. par le mot émycloíde qui, tout en représentant la même idée, est plus conforme aux usages généralement adoptés. Elle peut être caractérisée ainsi :

1. Verhandling over de natuurlijke geschiedenis der Nederl. overzeesche bezitingen, etc. fo Leyde, 18 \&. 
II. - 7 bis. Tortue Émydö̈de, T. emydoüdes, Sal. Müller.

T. emys, Sal. Müller. loc. cit.

Tortue Émyoïde, T. emydoïdes, Dum. et A. Dum. Cat. des Rept. p. 4, no 7 bis.

Carapace d'un brun fauve, large, déprimée, aplatie sur la ligne médiane; centre de chaque plaque du disque finement granulé, et entouré de plusieurs stries concentriques, polygonales, coupées par des lignes peu saillantes, et dirigées vers chacun des angles de la plaque; nuchale plus large en arrière qu'en avant; sus-caudale double; membres antérieurs recouverts, en avant et en dessous, d'écailles volumineuses et épaisses; sur la face postérieure des membres pelviens, et surtout en bas, et à la plante des pieds, les écailles prennent la forme de grandes épines triangulaires et pyramidales; près de l'origine de la queue, des épines semblables, du milieu desquelles il en sort une beaucoup plus saillante que les autres.

Cette espèce vient, dans le Catalogue, immédiatement après la T. SILLon NÉE, à cause de certaines analogies de forme, d'apparence et de coloration. Comme chez cette dernière, qui est d'un brun jaunâtre plus clair, les plaques sont striées et les membres sont couverts d'une écaillure dont les pièces sont robustes. J'insisterai peu cependant sur les différences qui les distinguent, car en rappelant que cette T. à laquelle je compare la nouvelle espéce n'a pas de gros tubercules aux cuisses, ni de nuchale, et que sa plaque sus-caudale est simple, la confusion est impossible.

De toutes les autres Chersites, c'est la T. Polyphìme qui, par le notable aplatissement de sa carapace, se rapproche le plus de la T. ÉmYDö̈DE, dont elle se sépare, d'ailleurs, nettement par ces deux caractères, qu'elle a une écaillure lisse et une plaque sus-caudale simple.

La Tortue décrite par M. S. Müller n'atteint pas une très-grand e taille. Il indique, comme dimensions moyennes, $\mathrm{o}^{\mathrm{m}} 25$ en longueur, $\mathrm{o}^{\mathrm{m}} \mathrm{o} / 45$ pour la queue et $\mathrm{o}^{\mathrm{m}}$ ig en largeur. L'exemplaire que le Muséum doit à la générosité du Musée de Leyde est long de $o^{\mathrm{m}} 24$, et large de $o^{\mathrm{m}}$ r 9 . La longueur du plastron est de $\mathrm{o}^{\mathrm{m}} 23$. Il provient, comme les autres échantillons de ce Musée, de l'île de Sumatra, et en particulier, de la grande vallée que traverse la rivière Aneh, sur les bords mèmey de ce vaste cours d'eau. 


\section{TORTUES PALUDINES OU ELLDITES.}

Ces Tortues de marais qui tiennent le milieu, pour la structure, entre les Chersites d'une part, et d'autre part, les Potamites ou Trionyx, et les Tortues marines ou Thalassites, peuvent ètre caractérisées ainsi :

Carnpace déprimée; pattes égales en longueur, à doigts distincts, mobiles, palmés, surtout aux membres postérieurs; ongles pointus, souvent courbés, au nombre de plus de trois; mâchoires nues.

Elles constituent une grande famille très-riche en genres et en espèces, et qui s'est accrue, depuis quelques années, beaucoup plus que celle des Tortues terrestres. Aux soixante-quatorze espèces décrites dans l'Erpét. génér., il faut en joindre seize nouvelles bien distinctes, ce qui donne le total considérable de quatre-vingt.dix.

PREMIERE SOUS-FAMLLE DES ÉLODITES : IES CRYPTODĖRES.

Le plus grand nombre de ces espèces récentes appartient à la $1^{\text {re }}$ SousFamille, celle des Cry pтодѐnes, caractérisée par la rétractilité de la tète, qui se cache directement en arrière, entre les pattes.

\section{GENTE CISTUIE. CISTUDO. FLEMING.}

Ce genre, le premier de la division des Cryptodères, comprend une espèce nouvelle que le Musée de Paris ne possède pas. Elle appartient au Sousgenre des Cist. bäillantes dont le plastron, tronqué en avant, échancré en arrière, est à battants entr'ouverts, et ne peut jamais fermer complétement les ouvertures antérieure et postérieure de la boîte osseuse.

Cette espèce, qui est propre à l'Amérique septentrionale, a reçu, de M. Holbrook, le nom de C. DE Blanding, en l'honneur du naturaliste de ce nom qui l'a observée le premier. Elle est, dans le Nouveau Continent, le représentant de la Cistude d'Europe.

Elle se distingue de la CisT. de la Caroline par les particularités suivantes: $\mathrm{I}^{\circ}$ La mâchoire supérieure, au lieu de présenter, en avant, un petit cro- 
chet, est profondément échancrée. $-2^{\circ}$ Le bord antérieur de la carapace est à peine relevé. - $3^{\circ}$ Le sternum n'est jamais entier : il est toujours échancré en arrière. $-4^{\circ}$ Les dimensions relatives des deux portions dont le sternum se compose, ne sont pas les mêmes dans les deux espèces. Dans la C. de la Caroline, la postérieure est beaucoup plus grande que l'antérieure, car elle est à cette dernière dans le rapport de 3 I à 22 , tandis que dans la C. DE Blanding, cette disproportion est moins marquée, puisque ce rapport n'est que de 43 à $39 .-5^{\circ}$ Chez cette dernière enfin, le sternium ne rejoint pas le pourtour de la carapace, et l'animal ne peut 'pas s'enfermer complétement dans son enveloppe osseuse, comme dans une boîte.

Quant au système de coloration, il rappelle un peu celui de la Cistude d'Europe par les nombreuses taches jaunes, ponctuées ou linéaires, dont la carapace est parsemée.

Sa zone d'habitation est fort circonscrite : la seule localité où elle se rencontre est l'État d'Illinois et le territoire du Wisconsin où elle est, dit-on, abondante. C'est cependant d'après un seul individu que M. Holbrook a fondé cette espece.

GENIRE ENI IDE. LMTS. DUMERIL.

Ce genre, qui était déjà le plus nombreux, s'est bien plus enrichi que les autres : au lieu de 35 espèces qu'il comprenait, il en renferme maintenant 45. C'est surtout le groupe si considérable des Émydes Américaines qui s'est augmenté par la découverte de neuf espèces propres au Continent septentrional du Nouveau-Monde.

Deux de ces dernières (É. ARÉotéf. et É. DE BÉRARD) ne sont encore décrites que dans notre Calalogue, et les six àutres le sont dans le bel ouvrage de M. Holbrook sur l'Erpétologie de l'Amérique du Nord.

\section{A. Émydes Européennes.}

Avant ces Émydes Américaines, celles d'Europe doivent noüs occuper un moment. Leur étude, d'ailleurs, se rattache à un point très-intéressant de géographie zoologique. Je venx parler de la similitude parfaite de notre Émyde vulgaire ou E. Caspienne avec des individus trouvés au Japon.

Le Musée de T_eyde ayant donné à celui dé Paris, dans ces dernières années, 
des échantillons Japonais de cette espèce et de plusieurs autres, il a été permis de reconnaitre l'exactitude des assertions de M. Schlegel contenues dans le passage suivant de l'ingénieux et habile Essai sur la physionomie des Serpents, et cité par M. de Siebold, dans son intéressante Introduction à la Faune du Japon : "Les reptiles de cette contrée donnent lieu à une observation assez remarquable, en ce que les Sauriens et les Ophidiens appartiennent, sans exception, à des espèces qui ne se trouvent point en Europe; tandis qu'on observe, parmi les deux antres ordres de Reptiles, des races analogues de la mème espèce dans ces deux contrées : telles sont nos deux grenouilles et la rainette (Rana esculenta, temporaria et Hyla arborea) qui sont exactement les mèmes au Japon, puis notre Tortue vulgaire (Emys caspica) qui forme, au Japon, une variété locale constante. ”

La comparaison attentive de l'échantillon reçu du Musée de Leyde et des planches 8 et 9 (Chelon.) de la Faune Japonaise, ainsi que du dessin ultérieurement publié par MI. Schlegel (Abbildungen neuer Amphib., pl. 46), ne laisse aucun doute sur la similitude extrème des individus rapportés du Japon, et de l'É. caspierre qui vit non-seulement dans les pays voisins de la mer dont elle porte le nom, mais aussi dans la Dalmatie et dans la Morée.

Cette Élodite a donc pris rang, dans le Catal., à la suite de l'espèce à laquelle elle appartient véritablement, et elle y est nommée :

III.-Émye caspienne, Var. Japonaise. E. caspica, Var. Japonica Schlegel.

La longueur proportionnelle plus considérable de la queue est la seule différence à noter; celle du système de coloration a peu d'importance.

Si l'opinion de M. Schlegel relative à cette variété doit être adoptée sans restriction, il ne semble pas possible d'admettre avec lui qu'une autre Émyde, dont un exemplaire a été donné au Musée de Paris par celui de Leyde, ne soit, comme le pense ce savant naturaliste, qu'une troisième variété, également Japonaise, de l'É. Caspienve.

Les caractères tirés de la conformation et des couleurs sont trop nets, pour que cette Tortue puisse ètre nommée, ainsi qu'il le propose, $E$. vulgaris (caspica), Var. Japonica picta. Aussi est-elle décrite dans le Catal. sous le nom suivant: 
1V. - 1 bis. Émyde Japonaise, E. japonica, Dum. et A. Dum.

Émye japonaise, E. japonica, Dum., et A. Dum., Catal. méth. des Rept., p. 8, no 1 bis.

En raison de la ressemblance entre cette espèce et celle dont, selon M. Schlegel, elle ne serait qu'une variété constante de climat, il est préférable, pour la bien faire connaitre, d'énoncer les caractères sous une forme comparative.

Ainsi on peut en donner cette diagnose :

Carapace à trois carènes assez saillantes, et dont la médiane l'est plus que les latérales; tête plus volumineuse, un peu plus épaisse; museau plus court; disque plus effile et plus tectiforme, à bords latéraux à peine relevés; plaques antérieures du plastron moins petites.

Ces particularités suffisent déjà comme moyens de distinction, mais ceux que fournit le système de coloration doivent aussi entrer en ligne de compte. Au lieu d'avoir une teinte olivâtre, la carapace est d'un brun noir assez foncé La gorge, les joues et le cou, si ce n'est à sa partie supérieure, sont ornés d'une quantité de lignes jaunâtres qui sont disposées, sur le cou, en longues bandes; à la gorge et sur les joues, elles se courbent et s'enroulent pour former des dessins isolés et détachés.

On trouve également, sous la queue et sous les pattes, de semblables lignes de couleur claire, mais qui souvent s'effacent.

Le plastron est nuancé de brun jaunâtre sur les côtés, ou à son extrémité antérieure.

La taille de cette Émyde est semblable à celle de l'Émyde CaSPIEnne. 


\section{B. Émydes Américaines.}

Je reviens maintenant aux espèces d'Amérique :

Elles sont au nombre de vingt-neuf, dont trois seulement appartiennent au continent méridional ${ }^{\mathrm{I}}$ : ce sont les E. Ponctulaire, E. punctularia, Schweigger, E. Marbrér, E. marmorea, Spix, que le Muséum ne possède pas, et sur l'identité de laquelle on peut conserver des doutes, et l'É. DE D'Orbigny, E. Dorbignii, Dum. Bib.

Une quatrième espèce, l'É. croıséE, E. decussata, Bell, doit, sans doute, être jointe aux précédentes, car elle n'est peut-être pas spéciale aux Antilles, quoique ses représentants dans les. Musées de Paris et de Iondres șoient tous originaires de ces îles et, en particulier, de Haiti, de Cuba et de la Guadeloupe.

Parmi les vingt-cinq Émydes de l'Amérique du Nord, j’en comprends une qui provient de l'Amérique centrale, c'est l'É. ARÉoléE, E. areolata, A. Dum., la faune de cette région du Nouveau-Monde offrant la plus grande analogie avec celle du Mexique, comme l'ont montré les intéressantes récoltes faites par M. Arthur Morelet dans le Guatemala, et spécialement dans la province de Peten.

Pour mettre en évidence les analogies et les différences qui existent entre ces vingt-cinq espèces, j’ai dressé le tableau analytique ci-joint, qui est un démembrement de celui que mon père et Bibron ont consigné dans l'Erpét. génér., tom. II, p. 234, dans le but de faciliter la détermination de toutes les espéces rapportées au genre Émyde. J'ai distrait de ce tableau celles dont il s'agit ici, et'j'y ai joint les neuf espèces non mentionnées dans cet ouvrage.

1. Parmi les seize autres espèces qui complètent le nombre de quarante-cinq que comprend le genre, il y en a douze Indiennes, une Africaine, et trois Européennes, l'E. JAPoNaise étant laissée dans ce demier groupe, à cause de ses rapports avec l'É. Caspienne, afin de montrer le lien qui l'unit à celle-ci, et pour le cas où, par l'examen ultérieur d'autres individus, on viendrait à ne la considérer que comme une variété. - Le Catalogue indique seulement vingt-huit espèces Américaines, et non pas vingt-neuf, puis, par suite, quarante-quatre Emydes en tout, au lieu de quarante-cinq, parce que l'Émyde DE L’OnÉGoy que le Muséum ne possede pas encore n’y est point enregistrée. 
DESCRIPTION DES REPTILES DU MUSÉUM.

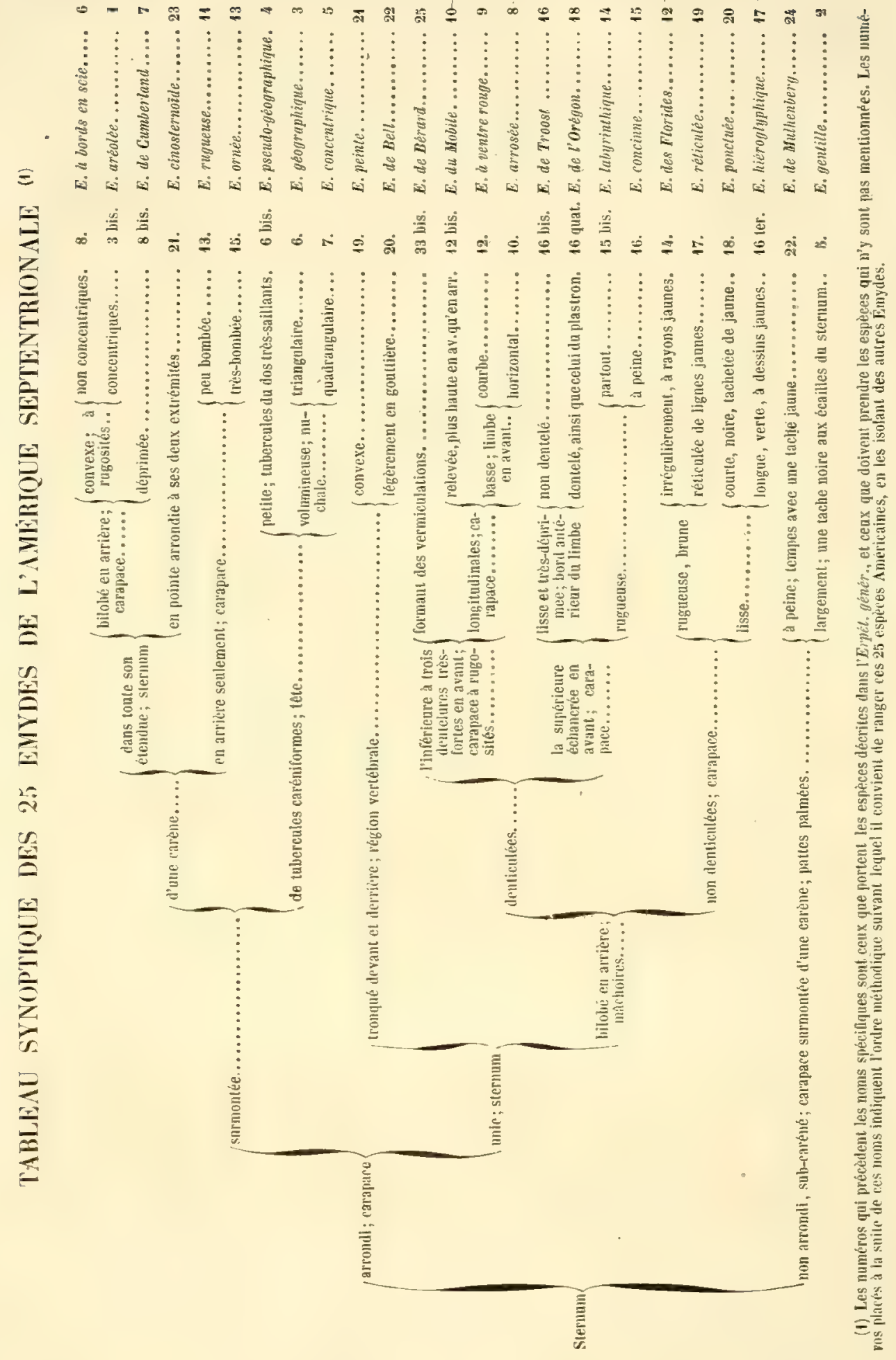


Aucune particularité nouvelle n'est à indiquer pour la plupart des. Émydes mentionnées sur ce tableat. Je ne décrirai que les neuf espèces qui portent des $\mathrm{n}^{\mathrm{os}}$ hors série, et quant aux autres, je parlerai seulement de celles dont l'histoire peut réclamer quelques nouveaux détails.

V. -3 bis. É. ARÉnLté, $E$. areolata, A. Duméril. ESPÈCE NOUVElle.

E. ARÉolée, E. areolata, A. Dum., Catal. méth. des Rept, p. $10^{4}$.

Carapace d'un vert brunâtre uniforme, très-peu déprimée, faiblement carénée, à extrémité antérieure plus étroite que lá postérieure, où le limbe, à peine relevé, est un peu dentelé et échancré au-dessus de la queue; plaques du disgue et du limbe creusées de stries concentriques; plastron à bords arrondis à sa jonction avec la carapace, très-long, entier en avant. faiblement échancré en arrière; tête petite.

Cette ĖMrDe, qui parait adulte, en raison de certaines irrégularités de son enveloppe osseuse où l'on voit comme des traces d'anciennes blessures, offre une conformation assez remarquable. Par son peu de dépression d'abord, elle a quelque analogie avecles Chersites ou avec celles des Paludines:qui diffèrent le moins des précédentes. Elle leur ressemble aussi par le peu de développement des membres et surtout des doigts, particulièrement de ceux des membres antérieurs, qui sont courts, fort peu palmés et, jusqu'à un certain point, disposés en moignon, comme dans les Tortues terrestres. Cependant l'arrangement même de ces doigts et la palmure des postérieurs ne ląissent aucun doute sur le genre de vie aquatique de ce Chélonien qui doit prendre rang au commencement du groupe des Élodites.

11 est facilement reconnaissable, entre toutes les Émydes, par l'aspect régulier des rugosités de sa carapace. Sur chaque plaque costale, près de sa jonction avec les vertébrales, on voit.une aréole entourée de stries concentriques, paralleles entre elles et aux bords de la plaque, et qui, par leur jonction angulaire à leurs extrémités, reproduisent la forme de cette plaque. Chaque

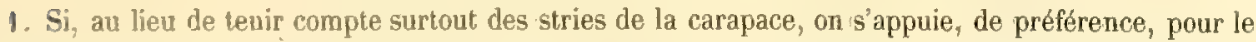
classement, sur la conformation des pattes, dont les doigts, peu développés et faiblement palmés, ressemblent à ceux des Cistudes et des premières espèces du genre Émyde, on voit que l'espèce nonvelle, dont il s'agit, a des rapports naturels avec l'É. ponctulaire. Il est donc préférable peut-être, de la placer á la suite de cette dernière, avec le $\mathrm{n}^{0} 3$ bis, plutôt que de la laisser, comme dans le Catalogive, aprés l'E. A lignes concentrioues, sous le $n^{0} 7$ bis. 
pièce du limbe porte une aréole semblable, située à son angle postérieur et inférieur, et entourée, en dessus et à sa partie antérieure, de lignes rugueuses qui forment, sur chacune de ces plaques marginales, une série d'angles rec. tangulaires, emboîtés les uns dans les autres, et dont le sommet est dirigé en haut et en avant. Les lignes rugueuses des plaques vertébrales ont moins de régularité.

$\mathrm{Si}$, par les particularités qui précèdent, cette ÉMxDE ressemble surtont à l'É. A Lignes concentrigues, elle s'en distingue, d'ailleurs, très-facilement par la dépression bien moindre de la carapace, et par le petit volume proportionnel de la tête.

C'est, en définitive, avec l'Ém. Ponctulaire qu'elle a le plus de rapports : par la convexité de la carapace, par la carène médiane qui la surmonte, par les petites dimensions de la tête, par les deux lignes jaunes, et peut-être rougeâtres pendant la vie, de la région supérieure du crâne, par la teinte jaune, ponctuée de noir, du cou et des membres, et enfin, par l'analogie de taille. Les caractères distinctifs, d'un autre côté, sont très-apparents : ils se tirent de l'étroitesse de la région antérieure du disque comparée à la postérieure; des rugosités concentriques de l'écaillure, et dont on ne trouve point de traces dans l'É. ponctularre; de la saillie moins prononcée de la carène médiane; de la longueur remarquable du plastron, qui est presque égale à celle du disque, et enfin du système de coloration de la boîte osseuse. La nouvelle espèce, en effet, est d'un brun verdâtre, et non tirant sur le rouge, comme cela se remarque dans l'autre Émyde ; le plastron, en outre, est moins uniformément brun.

Longueur de la carapace $0^{\text {m }} 155$; largeur à la région brachiale $0^{\boxplus} 085$, à la région fémorale $o^{m}$ ogo; long. du plastron $o^{m} 148$, de la tète $0^{m} 03_{2}$, larg. à la rég. temporale $\mathrm{o}^{\mathrm{m}} \mathrm{u}_{2} \mathrm{I}$; long. de la queue, $\mathrm{o}^{\mathrm{m}} 027$.

Ce Chélonien était compris parmi les beaux présents que M. Arthur Morelet a faits au Muséum, à son retour de l'A mérique centrale. Elle a été prise dans la province de Péten.

Quoique unique, elle a des caractères assez tranchés, pour qu'il ait été facile de reconnaître qu'elle n'appartient à aucune des espèces déjà décrites, et qu'elle devait devenir le TrPE d'une espèce nouvelle. 
VI. - 6 bis. Émide PSEUdo-géographiQue, E. pseudo-geographica, Lesueur.

Emys, sans nom spécifique, Lesueur, Mém. du Muséum, t. XV, p. 267 (1827).

Testudo geograph. Variety A. Leconte Ann. Lyc. nat. hist., t. III, p. 110.

E. pseudo-geogr., Lesueur, Mss. cités par Dum. et Bib. Erpét. génér., t. II, p. 256.

E. pseudo-geogr., Holbrook, N. Amer, herpet, t. I, p. 103, pl. xv.

E. pseudo-yeogr., Dekay, Zool. of N. York, Rept., p. 49, pl. II, fig. 3.

Émide Pзetdo-GÉogr., E. pseudo-geogr., Dum. et A. Dum., Catal. méth. des Rept., p. 9, nº 6 bis.

Téle petite, ovale; máchoire supérieure légèrement échancrée en avant, pour recevoir un petit crochet médian de linférieure; carapacé faiblement déprimée, dentelée postérieurement, échancrée à son bord antêrieur, assez lisse, si ce n'est sur les plaques costales qui portent de fines stries longitudinales; plaques vertébraies surmontées chacune d'un tubercule caréniforme trèssaillant.

Malgré la fort grande analogie qui existe entre cette espèce, que Lesueur avait presque admise, et l'E. GÉograpHIQUe, il est impossible de les prendre l'une pour l'autre. Le grand nombre d'échantillons réunis au Muséum démontre, de la manière la plus évidente, que cette nouvelle espèce diffère beaucoup de l'autre, dont elle n'est ni une variété, ni le jeune âge. On en a la preuve par la saillie des tubercules des plaques vertébrales, par la forme des mâchoires et par les petites dimensions de la tête, qui n'a que la moitié de la largeur qu'elle présente dans l'É. GÉOGR., dont la carapace est proportionnellement moins longue et moins élevée.

Sa distribution géographique n'est pas non plus tout à fait la même : sa zone d'habitation est moins étendue; on ne la trouve, en effet, mais en grande abondance à la vérité, que dans les affluents du Mississipi, et jamais sur le versant oriental des monts Alleghany.

Quant au système de coloration, il diffère trop peu de celui de l'Émyde à laquelle celle-ci est la plus comparable, pour qu'il soit nécessaire de le décrire. La carapace de quelques individus de cette nouvelle espèce cependant, au lieu d'ètre d'un vert jaunâtre, a une teinte brune, tirant un peu sur le jaune: c'est un spécimen ainsi coloré dont M. Holbrook a donné la représentation sur la $\mathrm{pl} .15$.

La taille de cette espèce est très-analogue à celle de l'espèce avec laquelle elle avait été d'abord confondue.

Le Muséum en possède une série d'âges divers duè à Lesueur, qui avait ArChives du Muséum. T. VI. 
recueilli ces individus aux États-Unis, dans le voisinage de la rivière Wabash, l'un des affluents de l'Ohio.

Parmi les Émydes américaines, il en est plusieurs dont le limbe porte, en arrière, des dentelures plus ou moins profondes. Aussi le nom d'Ém. à bords en scie, que Schweigger a imposé à l'une d'elles, conviendrait-il à d'autres, si cette espèce, en particulier, n’offrait certains caractères établis par ce naturaliste, et rappelés dans l'Erpét. génér., lesquels sont propres à la distinguer de ses congénères. Au nombre de celles-ci, le Muséum en renferme une : l'Ém. du Cumbercand, qui y était inconnue à l'époque de la publication du tome II de cet ouvrage, et qui ressemble plus que toutes les autres à l'ÉM. A BOROS EN SCIE. Elle a été nommée et décrite par M. Holbrook, et il résulte de cette description et de la figure qui l'accompagne, mais surtout de l'examen des échantillons maintenant rassemblés dans la Collection de Paris, que c'est une espèce distincte. Elle doit, dans la Méthode, prendre rang auprès de la précédente, et sous le $\mathrm{n}^{\circ} 8$ bis.

VII. - 8 bis. Émyde du Cumberland, Emys Cumberlandensis. Holbrook.

Holbrook, $N$. Americ. herpet., t. I, p. 115, pl. 18.

E. du Cumberland, Cumberlandensis, Dum. et A. Dum., Catal. method. des Rept, p. 13, $\mathrm{n}^{\circ} 8$ bis.

Carapace plus ou moins rugueuse, déprimée, d'une forme oblongue, souvent subquadrangulaire, mais à angles arrondis, munie, dans toute sa longueur, d'une carène peu apparente, léyèrement entrallée à son bord antérieur, et élégamment dentelée en arrière; plastron arrondi en avant, échancré à son extrémité caudale; une tache couleur de citron, et souvent d'un beau ronge, derriere l'orbite.

La forme de la carapace n'est pas absolument la même chez tous les individus : elle est plus souvent ovalaire qu'elle n'est subquadrangulaire, mais cette dernière conformation, jointe à la dépression qui l'accompagne, établit une différence assez tranchée avec la plupart des autres Tortues de ce groupe. C'est cependant bien à l'espèce actuelle que ces échantillons subquadrangulaires appartiennent. Par suite de ces dissemblances et de ce défaut d'uniformité, l'Éx. ou Comberland est, parmi les Émydes Américaines, si semblables entre elles sous tant de rapports, une des plus difficiles à déterminer.

Il faut ajouter, selon la remarque de M. Holbrook, que les rugosités de la carapace disparaissent avec l'âge. 
La tète est de volume médiocre; la màchoire supérieure porte, au milieu, une légère échancrure destinée à recevoir une saillie médiane de l'inférieure; le museau est court et légèrement pointu.

Le système de coloration de la carapace consiste en une teinte brun-verdâtre; il y a, sur chacune des plaques, une tache sinueuse ou des lignes rayonnantes jaunes ou fauves. Tout le disque est quelquefois d'une nuance foncée uniforme, où ces dessins se distinguent à peine. Le plastron est jaune et porte, le plus habituellement, vers le bord externe de chacune de ses plaques, une tache brune, ou, çà et là, des maculatures de la même couleur. La tête, brune en dessus, est ornée de petites lignes jaunes s'étendant jusqu'au cou; derrière l'orbite, et de chaque còté, l'on voit une tache couleur de citron, et souvent d'un beau rouge, longue de $0^{\mathrm{m}} \mathrm{o}_{2}$ à $\mathrm{o}^{\mathrm{m}} \mathrm{o} 3$, plus large en arrière qu'en avant.

Malgré une certaine ressemblance entre l'ÉM. A BoRDS EN SCIE et celle qui vient d'ètre décrite, cette dermère se distingue facilement par la dépression beancoup plus considérable de sa carapace que sa forme souvent subquadrangulaire rend d'ailleurs assez remarquable, et par sa tache temporale dont l'éclat est surtout très-vif avant l'état adulte, comme on s'en est assuré à la ménagerie où de jeunes sujets ont été plusieurs fois observés vivants.

L'état de Tenessee, aux États-Unis, est le seul, d'après M. Holbrook, où elle ait été observée jusqu'ici, quoiqu'il soit probable qu'elle habite d'autres Etats occidentaux de I'Union.

Le nom spécifique de cette Élodite est celui de l'affluent de l'Ohio, sur les rives duquel on l'a trouvée.

Le Muséum en contient une belle suite d'âges divers.

Sous les $\mathrm{n}^{\circ 3} \mathrm{I}_{2}$ et 13 , les auteurs de l'Erpét. génér. ont décrit, d'après Leconte, l'É. a ventre rouge, et, d'après Shaw, l'É. Rugueuse.

M. le Prince de Canino n'avait pas admis de distinction entre ces deux espèces, et, depuis lors, M. Holbrook a fait de même, et cependant il est impossible de ne pas reconnaître, sous ces deux noms, deux espèces différentes.

Ainsi que le naturaliste Américain le fait remarquer, les dissemblances dans le systeme de coloration ne fournissent pas un moyen suffisant de distinction, car elles sont peu tranchées. 
Celles qui se voient dans la conformation de la carapace sont plus importantes et manifestes. Elle est plus allongée, et son bord antérieur est plus. arrondi chez l'É. a ventre nouge que chez l'autre, dont le limbe, dans cette région, est presque horizontal; il en résulte que, chez cette dernière, la plaque nuchale a moins de longueur.

En continuant à intercaler parmi les Émydes décrites dans l'Erpét. génér. les espèces Américaines nouvelles, et en suivant un ordre méthodique, afin de les inscrire ici, comme l'indiquent d'ailleurs les $n^{\circ s}$ qu'elles portent, à la place qui leur est assignée par suite de l'examen comparatif auquel je les ai soumises, je range à la suite de l'É. a Ventre ROUGE, c'est-à-dire sous le $\mathrm{n}^{\circ}$ r 2 bis, l'É. DU Mobile.

VIII. - 12 bis. EMrYye DU MOBILE, Em)s Mobilensis, Holbrook.

Holbrook, N. Americ herpet. tome I, p. 71, pl. Ix.

Euyde Dо мовіLe, Emys Mobilensis, Dum. et A. Dum., Catal. méth. des Rept., p. 11, no 12 bis.

Carapace ovalaire, rugueuse, sans carène, convexe, relevée en avant, et déprimée en arrière où elle est échançrće et un peu dentelée; plastron oblong, plein et entier à son extrémité antérieure, échancré à son bord caudal; tète assezvolumineuse; museau pointu; máchoire supérieure dentelée dans toute son éfendue, ainsi que l'inférieure qui porte, à sa région moyenne, une sorte de croc."

Les plaques vertébrales et costales sont d'un brun verdâtre et réticulées de jaune; les marginales sont de la mème teinte. Chacune de ces dernières porte une ligne jaune qui, partant du milieu de son bord inférieur, monte jusque vers la région médiane de la plaque et, là, se bifurque : l'une de ses divisions se dirige en avant et l'autre en arrière, et comme l'une et l'autre rejoignent les divisions semblables de la plaque précédente et de la plaque suivante, il en résulte sur tout le limbe un dessin en forme de feston.

Le sternum est jaune, ainsi que la face inférieure des plaques limbaires où se voit, sur chacune, au niveau de sa jonction avec la plaque voisine, une grande tache noire dont le centre est marqué de jaune. Il y a, sur la tète et sur le cou, des lignes jaunes.

M. Holbrook fait observer, avec raison, que de toutes les É. de l'A mérique du Nurd, J'É. a ventre rodge est l'espèce qui ressemble le plus à celle-ci dont les différences spécifiques sont cependant bien manifestes. Ainsi l'É. ou 
Mовгце a les deux mâchoires dentelées et non pas seulement l'inférieure; la carapace n'est pas comprimée sur les còtés, elle est plus élevée en avant, plus aplatie et plus large en arrière; ses couleurs ne sont pas relevées par des lignes et par des taches rougeâtres; elle peut devenir une fois plus grande, et, enfin, elle n'a été rencontrée, jusqu'ici, quie dans l'État d'Alabama, sur les rives du Mobile, d'où le nom par lequel M. Leconte d'abord, puis M. Holbrook, l'ont désignée, et qui est celui qu'on lui donne sur les marchés de la Nouvelle-Orléans.

Les échantillons du Muséum sont dus à M. Harlan, et la Ménagerie a possédé vivant un bel exemplaire adressé de la Nouvelle-Orléans.

Nous plaçons près de l'É. ORvéE, sous le $\mathrm{n}^{0}$ i5 bis, une Émyde non encore décrite, mais dont la distinction avait été établie par Lesueur. Il lui avait donné le nom d'É. LAbyrinthique, sous lequel elle est inscrite dans le Catalogue où elle occupe, au $n^{\circ} 22$ ter, un rang qui lui avait été assigné provisoirement et qui n'a pas été maintenu.

IX. - I5 bis. Limpe Labyrinthrede, Emys labyrinthica, Lesueur.

- Emys labyrinthica, Lesueur, uss.

Idem, Dum. et A. Dum., Catal. méth. des Rept., p. 13.

Carapace ruyueuse, assez régulièrement ovalaire, bombée, surtout sur la ligne média ne, mais sans carène, à bord postérieur un peu relevé et sinueux; plastron entier en avant, échancré en arrière; tele assez columineuse; máchoire inférieure munie d'un crochet correspondant à un enfoncement mérian de la mâchoire supérieure.

Quoiqu'il y ait un certain rapport entre cette espèce et l'ÉM. onnéE, elle peut cependant en être assez facilement distinguée, surtout par le systẹme de coloration. Il consiste, ici, en une teinte verte, plus ou moins brunâtre, ornée d'un grand nombre de lignes jaunes, dont la direction sinueuse et l'enroulement sur le limbe, en particulier, a valu à cette espèce le nom de labyrinthique.

Le Muséum en a reçu plusieurs exemplaires recueillis par Lesueur sur les bords de la rivière Wabash, anx États-Unis. 


\section{X. - I6 ter. Émxde hí́roglyphiQ̨e, Emys hieroglyphica, Holbrook.}

Holbrook, $N$. Amer. herpet., tomẻ I, p. 111, pl. xvii.

Émyde hiéroglypheue, Emys hieroglyphica, Dum. et A. Dum., Catal. méth. des Rept., p. 12 '.

Carapace en forme dovale fort allongé, se terminant presque en pointe à son extrémité postérieure où elle est incomplétement dentelée, très-déprimée, sans carène, lisse; plastron oblong, échancré en arrière; tête remarquablement petite et étroite, à museau un peu pointu.

Cette diagnose où sont relevés les caractères tirés de la forme allongée et de l'aplatissement de la carapace, ne permet la confusion de cette espéce avec aucune de ses congénères. Le petit volume de la tête est aussi une particularité digne de remarque. Il n'y a pas de dentelures aux mâchoires, dont la supérieure porte, à la région médiane, une petite échancrure pour recevoir la saillie correspondante de l'inférieure. Les trois ongles du milieu, aux pattes de devant, l'emportent de beaucoup sur les autres par leurs dimensions et par leur force.

La teinte générale du disque est un vert-olive assez clair où de larges lignes jaunes circonscrivent des espaces de formes et de dimensions variées, et dans chacun desquels se voient des lignes plus étroites, égálement jaunes. Des taches et des lignes de nuance semblable forment, sur le limbe, des dessins analogues, par leur aspect, aux caractères hiéroglyphiques.

Le plastron, d'un jaune verdâtre, est plus ou moins garni de taches sombres. La tête est d'un brun foncé, relevé par de nombreuses lignes jaunes prolongées sur le cou.

Cette Émyde est d'une assez grande taille, qui produit un contraste frappant avec son peu d'élévation.

Elle vit, aux Ėtats-Unis, dans les eaux des États de l'Ouest. Le mâle et la femelle que le Muséum possède ont été recueillis dans la Caroline du Sud et sont dus à M. Harlan.

1. Le $\mathrm{n}^{\circ}$ 22 bis qui a été donné à cette espèce, dans le Catal, doit être remplacé par celui qu'elle porte ici; ce dernier la rapproche des E. Américaines les plus déprimées, c'est-à-dire des E. oE TroosT ( 16 bis) et DE L'ORÉGON (16 quater). 
XI. - 33 bis. Émyde de Bérard, Emys Berardii, A. Dum. espèce nodvelle.

ÉuYde de Bérard, Emys Berardii, Catal. méth. des Rept., p. 11, nº 33 bis.

Carapace d'un brun jaunatre, couverte de fines rugosités irrégulières, formant une multitude de petites rermiculations, allongée, sub-ovalaire, mais resserrée et étroite au-dessus des membres antérieurs où le limbe s'incline en bas, tandis qu'il est relevé et un peu sinueux en arrière; première et dernière plaques vertébrales surmontées d'un tubercule caréniforme peu saillant; mâchoires dentelées.

De toutes les Émydes, il n'en est aucune à laquelle elle ressemble plus par sa conformation générale et par sa grande taille qu'à l'ÉM. A Trors Bandes décrite dans l'Erpét. génér. sous le $\mathrm{n}^{0} 33^{\mathrm{I}}$. Par les nombreuses vermiculations de sa carapace, par l'absence des trois bandes noires longitudinales, et surtout par la différence d'origine, puisque la précédente est Indienne, tandis que l'É. DE BÉRARD est Américaine, la confusion est impossible. Ces mêmes caractères établissent également une différence fort tranchée entre cette nouvelle Émyde et toutes les espèces déjà connues ${ }^{2}$.

Les plaques du disque, à l'exception de la première et de la cinquième, qui sont surmontées d'un tubercule caréniforme, n'offrent rien de particulier à noter. Celles du limbe sont très-inégales en hauteur : elles vont en augmentant, depuis les collaires, qui sont les plus basses, jusqu'à la région fémorale où leur élévation est une fois plus considérable; puis, au delà, elles diminuent d'une façon presque insensible. La nuchale est petite et presque carrée.

Par suite d'usure, le limbe est un peu dentelé à son bord postérieur, mais il est probable qu'à l'état normal, il est uni. Il est, d'ailleurs, plus relevé

I. Des deux individus de l'espèce nouvelle que le Muséum possède, l'un est indiqué comme provenant de l'Amér. mérid., mais le fait est douteux : il est dủ au lieutenant Maw. Par une erreur d'étiquette, la même origine a été attribuée, dans le Catalogue, à l'autre spécimen, tandis qu'il est positif que M. le Capitaine Bérard l'a rapporté des eaux douces de Vera-Cruz (Mexique).

De ces faits, il résulte que l'espece dont il s'agit ne doit pas conserver le $n^{n} 9$ bis qu'elle porte dans le Catalogue, mais qu'elle doit prendre le no 33 bis.

2. Une faute d'impression dans le Catalogue, p. 11, lig. 17, est importante à corriger : ce n'est pas quatre ongles en avant et cinq en arrière, qu'il faut lire, mais cinq en avant et quatre en arrière caractëre générique des Émydes). 
dans cette région chez le plus grand des deux individus, qui paraît être un mâle. L'autre, dont le Muséum ne possède malheureusement que la boîte osseuse, est de taille un pen moindré, et par suite, sans doute, de la différence de sexe, il est moins allongé. En outre, la région brachiale est moins resserrée; du reste, cet individu est très-semblable au précédent. Toute la carapace cependant est proportionnellement plus relevée, parce que le sternum est moins plat, et il n'offre pas, comme chez le mâle, vers son extrémité postérieure, l'excavation caractéristique de ce sexe.

Le système de coloration consiste en une teinte générale et uniforme d'un brun fauve, plus jaunâtre sur la carapace, que nous supposons avoir appartenu à un individu femelle.

Le plastron, sur les deux échantillons, est jaunâtre et sans taches.

Les dimensions du plus grand sont les suivantes:

Longueur de la tête, $o^{\mathrm{m}} \mathrm{o} 8$; du cou, $\mathrm{o}^{\mathrm{m}} 11$; de la carapace, $\mathrm{o}^{\mathrm{m}} 47$; de la queue $\mathrm{o}^{\mathrm{m}} \mathrm{I}_{\mathrm{I}}$; largeur de la carapace, à la région brachiale, $\mathrm{o}^{\mathrm{m}} 25$, à la région fémorale, $o^{\mathrm{m}} 34$; longueur du plastron, $o^{\mathrm{m}} 33$.

La carapace de l'autre spécimen a $\mathrm{o}^{\mathrm{m}} 43$ en longueur; sa largeur, à la région brachiale, est de $\mathrm{o}^{\mathrm{m}} 27$, et à la région fémorale, de $o^{\mathrm{m}} 36$; le plastron est long de $0^{\text {m }} 33$.

Au nombre des É. de l'Amérique du Nord qui manquent au Musée de Paris, il faut comprendre l'Émyde Des Florıdes, E. Floridana, Leconte, décrite sous le $\mathbf{n}^{\circ} 14$ dans l'Erpélologie générale. Dans l'ouvrage de M. Holbrook, la description a été faite d'après nature, et elle est accompagnée d'une fig. (t. I, p. 65, pl. 8). Sans m'arrêter à rappeler ses caractères spécifiques, je dirai seulement que l'habile Erpétologiste dont je viens de citer le nom qui se rattache d'une manière si remarquable à l'bistoire des Reptiles des ÉtatsUnis, l'a trouvée parfaitement distincte, d'après les nombreux individus de sa collection.

Une autre espèce, inscrite dans le Catal. comme se trouvant dans les Collect. du Muséum, y manque cependant : c'est l'É. DE Troost, $E$. Troostii Holbrook.

Trompé par une étiquette apposée par Bibron, avant l'arrivée en France de l'ouvrage du naturaliste Américain, sur deux Émydes qui, suivant lui, appartenaient à cette espèce, je les ai admises comme ses véritables représentants, malgré certains défauts de coïncidence avec la description contenue dans le texte de N. Amer. herpetology. Une étude nouvelle de cette description et de 
ces prétendues É. DE Troost démontre qu'elles ne sont autre chose que des échantillons de l'É. a BORDS EN SCIE.

Les détails donnés dans le Catalogue, p. 1o, $\mathrm{n}^{\circ} 8$ bis, comme se rapportant à l'É. DE Troost, doivent donc ètre laissés de còté, et cette Émyde doit prendre rang sous le $\mathrm{n}^{\circ}, 6$ bis, après l'É. concrune qui la précède dans l'ouvrage de M. Holbrook.

Lne troisième espèce enfin, l'É. DE L'OrÉgon, E. Oregoniensis, Harlan, décrite par M. Holbrook (t. I, p. Io7, pl. 16), et primitivement par M. Harlan, qui l'a nommée (Amer. journ. of Aits and Sciences, t. XXXI, p. 382, pl. 3i), est inconnue à Paris.

Elle parait parfaitement distincte de toutes ses congénères par la dépression remarquable de sa carapace, par les dentelures du bord antérieur nonseulement du limbe, mais du plastron, et par les deux prolongements en forme de dents que porte sa mâchoire supérieure.

La figure donnée par M. Holbrook montre qu'elle a quelque analogie par l'allongement et Ja dépression de sa carapace avec l'E. HIÉRogLypHIQUE aupres de laquelle i] la place. Or, cette dernière portant, sur le tableau le $\mathrm{n}^{\circ}$ i 6 ter, l'É. DE L'OréGoN a dû prendre le no 16 quater.

Le Musénm espère que M. Holbrook pourra lui procurer ces curieuses espèces, dont l'absence, dans ses Collections, y laisse des vides si regrettables.

GEVRE TETIONIX. TETRAONYT. LESSON.

Ce genre diffère de celui des Émydes en ce que les espèces qu'on y rapporte ont seulement quatre ongles aux pattes antérieures. Le cinquième doigt ne manque pas, mais au lieu d'être apparent, comme dans le genre précédent, où l'un des caractères essentiels se tire de l'inégalité dans le nombre des ongles aux pattes de devant et à celles de derrière, il est entièrement caché sous les téguments.

Des deux espèces inscrites dans l'Erpét. génér., une seule, le T. DE LEsson, est connue au Muséum, et le T. Baska, établi d'après une des planches des Ind zool. illustrat. du général Hardwick, y manque.

Je parle ici de ces espèces, seulement afin de dire que M. Grax (Cat. of ARCAIVEs DE MCsérM. T. VI. 
Tortoises, p. 29) persiste dans l'opinion, anciennement émise par lui, que la seconde n'est qu'une variété de la première.

GENTE PUATISTENE. PLATEATERAON. GRAY.

Ce genre, dont le nom signifie à large plastron, est reconnaissable surtout au volume de la tête chez l'unique espèce qu'il renferme (P. MégacéPbaLE), dont le Muséum ne possède encore qu'un échantillon.

Aucun nouveau détail n'est à ajouter.

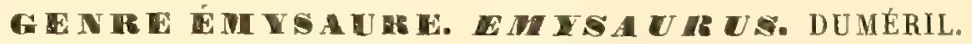

Ce genre est l'un des plus nettement caractérisés parmi les Élodites, par la forme en croix du plastron, dont la partie moyenne est excessivement étroite, par le volume considérable de la tète, par la courbure en crochet des mâchoires, par la longueur de la queue, et enfin, par un ensemble tout à fait spécial.

Depuis l'époque, déjà ancienne, où Schweigger a établi une coupe générique spéciale, sous le nom de Chelydra, pour la Tortue serpentine de Linné, cette espèce, jusqu'à ces dernières années, était unique.

Un bel individu, remarquable par ses instincts cruels et par l'avidité avec laquelle il se jetait sur sa proie, et en particulier sur les oiseaux qu'il commençait par noyer avant de les manger, a vécu à la Ménagerie pendant un grand nombre d'années.

On doit maintenant la connaissance d'une seconde espèce au Professeur Troost. Ce naturaliste, en adoptant, comme nom générique, la dénomination de Chélonure imposée, sans utilité, à ce genre par Fleming, l'a nommée Ch. Temminckii. Elle était également connue au Musée de Paris, à qui Lesueur en avait procuré un exemplaire après la publication du t. II de l'Erpét. génér. Cette belle et curieuse Élodite avait reçu de mon père et de Bibron le nom d'ÉnYsAUne LACERTINE qui n'a pu être conservé, puisque, avant toute publication en France, elle a été décrite et figurée par M. Holbrook, sous les noms donnés par M. Troost.

Elle est brièvement indiquée dans le Catalogue, mais elle mérite une description détaillée, en raison des particularités intéressantes qui la distinguent. 
XII. - 1 bis. Énysaure DE TEMmirck, Emys, Temminckii, Dum. et A. Dum.

Emisaure lacertive Dum, et Bib. Mss.

Chelonura Temm. Troost uss.

Chelonura Temm. Holbrook, $N$. Amer. herpet., t. I, p. 147, pl. 24.

Eursaure de Teumrack, Em. Temminckit, Dum. et A. Dum., Cat. des Rept., p. $15, n^{\circ} 1$ bis.

Tête énormément volumineuse, triangulaire, très-large en arrière, pointue en avant, couverte de plaques en dessus et sur les cótés; mâchoire supérieure robuste, en forme de bec de vautour, dont le crochet descend à angle droit; carapace très-fortement tri-carénée, concave en avant, profondément échancrée et dentelée en arrière; 31 plaques marginales disposées en deux rangs superposés sur les flancs; queue sans créte.

Cette diagnose, où sont exprimés les caractères remarquables de ce grand Chélonien, suffirait pour le distinguer, non-seulement dẹ toutes les espèces connues, mais aussi de l'autre Émysaure avec laquelle elle a des rapports assez marqués.

Les dissemblances sont cependant très-évidentes, comme le montrent les détails comparatifs suivants :

Dans cette espèce nouvelle, la carapace est moins déprimée, le limbe a plus de largeur, surtout sur les parties latérales où les plaques marginales sont sur deux rangs superposés, ce qui est une exception très-remarquable à la disposition habituelle. Ces plaques sont assez fortement convexes, d'où résulte, autour de la carapace, si ce n'est tout à fait en avant et en arrière, l'apparence d'une sorte de gros bourrelet.

La plaque nuchale, qui est fort large et fort basse, est en arc de cercle; aussi voit-on, dans ce point, un cintre dont les limites sont indiquées par la saillie que porte, à droite comme à gauche, la première plaque margino-collaire : cette saillie commence la carène latérale du disque. Le limbe, échancré au-dessus de la queue, a, de chaque côté de cette échancrure, quatre grandes dentelures. Les premières plaques vertébrales sont placées sur un plan horizontal auquel la cinquième est un peu inférieure. Leur ligne médiane forme une saillie très-élevée que commence un renflement de la nuchale. Les plaques costales, près de leur jonction avec les vertébrales, sont très-saillantes, et il résulte de cette conformation une haute carène; de sorte que la médiane est située entre deux autres carènes dont elle est séparée, d'un côté, 
comme de l'autre, par une profonde gouttière. En dehors de cette arète, les plaques costales sont brusquement inclinées en bas et en dehors.

Quand on compare ce test si accidenté par ces élévations et par ces enfoncements, à celui de l'É. SERPENTINE, on est frappé des différences notables qui existent entre ces deux espèces. En outre, dans l'É. DE TEM., la tète est beaucoup plus volumineuse; elle est couverte de plaques plus grandes, et en particulier, par une très-large occipitale; le crochet de la mâchoire supérieure est plus long et plus acéré; il en est de mème de celui qui termine, en avant et au milieu, la mâchoire inférieure, dont les bords sont tranchants et concaves. Le cou, dont la peau est granulée, avec des excroissances verruqueuses, cornées à leur pointe, a un volume plus considérable. La quene enfin est moins bien armée, car, au lieu d'une carène, elle n'est revètue, en dessus, que de plaques à peine saillantes. La forte palmure des pattes est semblable à ce qui s'observe chez sa congénère.

La teinte générale est un brun plus ou moins jaunàtre dans certains points.

L'unique échantillon par lequel cette précieuse Élodite est représentée au Muséum provient de Lesueur, qui l'avait rapportée des États-Unis. M. Holbrook dit qu'elle vit dans le Mississipi, et dans tous les cours d'eau que ce fleuve reçoit, ainsi que dans les eaux tributaires du golfe du Mexique.

Ce naturaliste dit, en outre, que les habitudes de cet animal, autant qu'on peut en juger, en raison de sa rareté, paraissent ètre les mèmes que celles de l'É. serpentine. On la rencontre dans les mêmes localités, et elle recherche la même nourriture. $M$. Troost a trouvé dans les intestins d'une de ces Tortues, des fragments d'os de poissons et des débris d'une grande espèce de mollusque du genre Unio.

Dimensions : Longueur de la carapace, $o^{m} 38$, de la tête, $o^{\text {m }}$ r 5 , du cou, $u^{m}$ i 3 , de la queve, $o^{m} 30$, du plastron, $0^{m} 29$; largeur de la carapace d'un flanc à l'autre, $\mathrm{o}^{\mathrm{m}} 34$, de la tète, $\mathrm{o}^{\mathrm{m}} 125$.

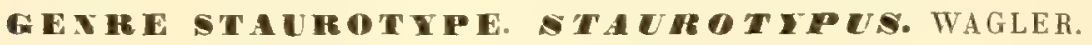

Parmi les Élodites Cryptodères, il y a trois genres dont les espèces ont le plastron plus ou moins mobile sur la carapace. Il a été question déjà du genre Cistude où cette mobilité est très-considérable, car la portion antérieure et la postérieure peuvent, à la volonté de l'animal, être rapprochées du limbe. 
Une disposition analogue, avec quelques différences cependant, comme il est dit plus loin, se voit dans le genre Cinosterne. Dans les Staurotypes, dont il s'agit ici, le sternum, ainsi que l'exprime la dénomination créée par Wagler, est cruciforme, et la portion antérieure du plastron peut, seule, être élevée ou abaissée; il est rare, du moins, que l'autre portion jouisse d'une légère mobilité. Entre les Cinosternes et les Staurotypes, qui se suivent dans la méthodé, il y a donc cette différence, que jamais, chez ces derners, il n'existe, à la région postérieure du sternum, la mobilité très-manifeste qui est constante chez les autres. La forme de ce plastron est, d'ailleurs, bien caractéristique, sa largeur, chez les Cinosternes, contrastant avec ce qui s'observe dans les Staurotypes.

Les deux Staurotypes décrits dans l'Erpét. génér. sont rangés par M. Gray (Cal. of Tort., p. 34) dans le genre Cinosterne, et M. le Prince de Canino y fait rentrer seulement le second de ces Staurotypes, c'est-à-dire, la T. ovorante de Daudin. M. Holbrook, à l'exemple de M. Th. Bell, laisse cette dernière dans le genre Sternothère, et il la décrit et la figure sous les noms de Stern. odoratus (N. Amer. herpet., t. I, p. I33, pl. 22).

Les observations qui précèdent, relativement aux caractères génériques des Staurotypes, montrent que les animaix qui portent ce nom, dans l'Erpét. génér., ne sont positivement pas des Cinosternes, et leur classement parmi les Cryptodères dit assez qu'ils doivent être éloignés des Sternothères, rangés arec raison dans la sous-famille des Élodites Pleurodères.

Quant aux espèces elles-mèmes, aucune particularité nouvelle n'est à mentionner, si ce n'est la remarque faite par M. Holbrook relativement à l'étendue de la zone d'habitation du St'Aur. nusqué: on le trouve, dans une circonscription géographique bien plus vaste que la plupart des autres Chéloniens de l'Amérique septentrionale, puisqu'au nord, il remonte jusqu'à l'État du Maine, qu'il est très-abondant dans les États du Sud, tels que la Floride et l'Alabama, et qu'enfin, à l'ouest des monts Alleghany, il habite les rivières du Cumberland et du Tenessee.

\section{GENTE CINOSTENUE. CINOSTERTON. WAGLER.}

Les Élodites de ce groupe sont bien nettement caractérisées par la mobilité de la portion antérieure du plastron et de la postérieure, qui se meuvent sur une pièce médiane fixe. 
Outre plusieurs différences notables, inutiles à rappeler ici, ce genre se distingue facilement de celui des Cistodes, nommées aussi Tortues à boîte, en raison des mouvements que peuvent exécuter les deux portions du plastron. Chez ces dernières, il n'y a pas de portion moyenne immobile comme dans les Cinosternes, et les deux battants du plastron, qui n'est fixé à la carapace que par un cartilage, se meuvent sur une mème charnière transversale et moyenne.

Des trois espèces décrites dans l'Erpét. génér., la troisième (C. HIRTIPÈDE, Wagl.) manque au Musée de Paris; dans celui de Londres, elle porte le nom de Oblong Swanka, Kinost. oblongum, Gray, (Cat. of Tort, p. 33). Son principal caractère consiste en l'absence de la plaque nuchale.

Les deux autrès espèces sont parfaitement distinctes l'une de l'autre, ainsi que des deux nouvelles espèces qu'on a observées vivantes à la Ménagerie, et dont mon père a fait faire à cette époque les dessins qui sont reproduits sur deux des planches ci-jointes.

\section{TABLEAU SYNOPTIQUE DES ESPĖCES DU GENRE CINOSTERNE 4.}

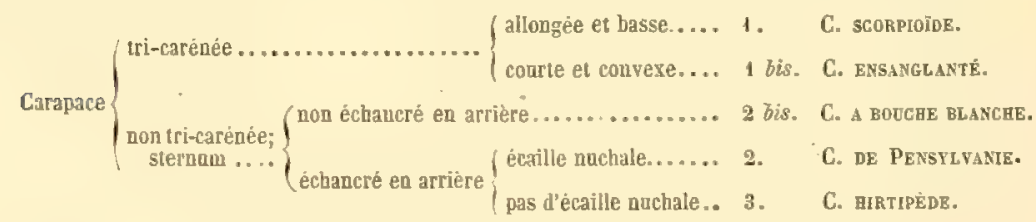

Ce tableau montre le rang que chacune des espèces doit prendre et les caractères les plus saillants propres à les distinguer de leurs congénères.

XIII. - I bis. C. ensanglanté, C. cruentatum, Dum., Bib. nouvelle espè Ce. Criosterve evsavglanté, C. cruentatum. Cat. des Rept. p. 16, no 1 bïs.

Carapace d'un brun rougeatre, tri-carénée, courte, fortement inclinée en bas, à son extrémité postérieure; sternum non échancré en arrière; téguments ornés de nombreuses maculatures rouges.

La présence de trois carènes longitudinales sur la carapace est une particularité qui, au premier abord, établit une similitude assez frappante entre

1. Ce tableau est destiné à remplacer celui de l'Erpét. génér., qui ne comprend que trois espèces. 


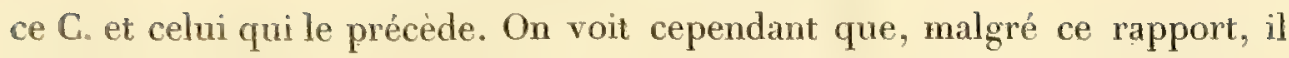
y. a des différences très-manifestes. La plus importante consiste dans la forme générale du disque : au lieu de représenter, comme celui du C. sconpioïı, une ellipse allongée et très-étroite, il est court, ramassé, et il offre une inclinaison brusque en arrière où il s'infléchit en bas presque perpendiculairement; en outre, il a une hauteur plus considérable. Le sternum enfin n'est pas échancré en arrière, tandis que chez le Cinost. qui sert ici de terme de comparaison, il existe toujours, dans cette région, une petite échancrure.

Il n'y a rien de spécial à noter relativement au système de coloration du test, dont la teinte est brune en dessus, et brun-jaunâtre en dessous, comme chez les autres espèces de ce genre, mais les pattes, le cou et la tète portent de nombreuses petites taches d'un rouge de sang, nettement détachées sur la couleur jaune-verdâtre de ces parties.

L'espèce est connue au Musée de Paris par l'échantillon unique observé vivant. Il avait été acquis, de sorte qu'il reste un peu d'incertitude sur sa patrie. On sait seulemènt qu'il est Américain, et très-probablement, il avait été recueilli dans le Continent septentrional, d'où l'on reçoit bien plus souvent des Reptiles pour la Ménagerie, que de l'Amérique du Sud.

Peut-ètre est-ce un représentant de cette espèce que M. Gray désignè dans son Cat. of '́ort., p. 33, en parlant d'un spécimen du C. scorpioïde reçu de l'Amérique centrale, et dont il dit: Dos très-convexe, arrondi.

L'autre espèce qui n'a pas trois carènes dorsales, offre surtout des rapports avec le C. Pensyluaneur. Il est facile cependant de l'en séparer, comme le prouve la diagnose suivante:

XIV. - 2 bis. C. bodche blanche, C. leucostomum, Dum., Bib. NOUVELLIE ESPÈCE.

Cinosterne botche blanche, C. leucostomum. Cat. des Rept., p. 17, 2 bis.

Carapace d'un brun rougeâtre, assez allongée, faiblenent uni-carénée; sternum non échancré en arriere.

Ce qui caractérise surtout cette espèce nouvelle, c'est la forme de la carapace, dont le limbe, en arrière, au lieu de se diriger presque verticalement en bas, est un peu relevé, de sorte que sa direction est presque semblable à sa région postérieure et à l'antérieure, ce qui n'a pas lieu dans le C. DE. PEN- 
SYLVANiE, où il est beaucoup moins horizontal au-dessus de la queue et des membres postérieurs qu'il ne l'est en avant. On ne remarque pas non plus, contrairement à ce qui s'observe le plus souvent, chez ce dernier, une dépression médiane du disque, et enfin, jamais le sternum n'est échancré à son extrémité caudale.

Le système de coloration a beancoup d'analogie avec celui de l'espèce voisine; mais chez presque tous les sujets, les mâchoires sont d'un blanc jaunâtre, sans taches, ce qui a valu à cette espèce le nom par lequel mon père et Bibron l'avaient désignée dans leurs manuscrits, et que nous avons conservé.

Les mêmes incertitudes que pour le C. ENSANGLAíté se présentent ici, relativement à l'origine de quelques-uns des échantillons de cette espèce. Elles portent sur ceux qui oǹt été acquis, et qu'on a observés vivants à la Ménagerie. Si, en effet, on a lieu de les croire recueillis dans l'Amérique du Nord, on sait, d'un autre côté, et d'une façon positive, qu'il en a été trouvé dans la Nouvelle-Grenade. L'un a été rapporté de la Vallée de la Madeleine, par M. J. Goudot, et un autre des environs de Santa-Fé de Bogota, par M. Lewy. L'espèce se rencontre aussi dans l'Amérique centrale, puisque M. Arthur Morelet en a donné un spécimen qu'il a pris sur les bords du Rio Sumasinta.

II SOUS-FAMILLE DES ÉLODITHS. LES PLEURODÉRES.

La seconde sous-famille des Tortues Élodites ou Paludines comprend toutes celles que Wagler a réunies par ce singulier caractère, que la tête et le long cou qui la supporte, au lieu d'être rétractiles, viennent horizontalement se plier et se coucher de côté dans l'espace plus ou moins étroit compris entre le bouclier et le plastron. Chez toutes, en outre, le bassin est soudé tout à la fois au plastron et à la carapace.

Les auteurs de l'Erpét. génér. ont décrit dans cette sous-famille sept genres dont le premier (genre peltocéphale, Peltocephalus Dum. et Bib.), où l'on ne compte encore qu'une espèce ( $P$. TRACAXA) remarquable par sa grosse tête et ses mâchoires crochues, n'a subi aucune modification, non plus que le genre Chécodine, Chelodina Fitzinger. Celui-ci est facilement reconnais- 
sable, dans les trois espèces qu'il comprend, par l'allongement et par l'aplatissement de la tête ainsi que par la très-grande longueur du cou.

Aucun changement, enfin, n'a été apporté au dernier genre de ce groupe, celui si remarquable des Chécrdes, Chelys. Dum. L'espèce unique qu'il comprend, nommée Ch. Matamata, est un des Chéloniens les plus curieux que l'on connaisse. Cette tortue, en effet, qui offre quelque analogie éloignée avec le singulier Batracien anoure connu sous le nom de Pipa, est caractérisée, de la façon la plus nette, par sa tète très-déprimée et triangulaire, par ses narines prolongées en trompe, par son énorme bouche garnie de replis cutanés simulant des lèvres, et par la triple carène longitudinale de sa carapace.

Les quatre antres genres de la sous-famille des Pleurodères doivent nous arrèter quelques moments.

GEMTE PODOCNEMIDE. PODOCNOMIA. WAGLER.

Le caractère principal de ce groupe, ainsi que le nom par lequel on le désigne, sont tirés de la présence des grandes écailles du talon. Les tortues qui y sont rapportées peuvent, d'ailleurs, être facilement reconnues par le large sillon longitudinal dont leur front est creusé. Il ne renferme; dans l'Erpét. génér. que deux espèces, les seules qui fussent décrites jusqu'alors. Quoique fort voisines l'une de l'autre, elles se distinguent aisément par la forme de la carapace, qui est beaucoup plus large et plus déprimée dans l'espéce dite P. Élargie ( $P$. expansa), que dans la P. de Duméril ( $P$. Dumeriliana.)

Une troisième doit maintenant être ajoutée à ces dernières, et nous l'avons nommée, en la dédiant au voyageur qui l'a recueillie dans les environs de Santa-Fé de Bogota, Podocnémide de Lewy (P. Lewyana). A. Dum.

Les caractères propres au genre, et dont nous venons d'indiquer les plus saillants, sont très-évidents chez cette Tortue et chez une autre, plus jeune, qui appartient, depuis un certain nombre d'années, à la collection du Musée de Paris, mais que nous détachons de la P. DE DuM., à laquelle elle avait été rapportée, pour la rapprocher de la nouvelle $\mathrm{P}$., car elle lui est semblable. Quant aux caractères spécifiques, ils sont assez nets pour motiver l'établissement d'une troisième division dans le groupe générique. 
Cette nouvelle P. n'est pas mentionnée dans le Calalogue, dont la première livraison était déjà imprimée à l'époque du retour en France de M. Lewy.

\section{XV. - 5 bis. Podocnémide de Lewy, Podocnemis Lewyana, A. Dum. espèce nouvelte. (Planches xviII et xIX).}

Carapace ovale, entière, très-fortement déprimée, sans aucune saillie sur la ligne médiane; régions fémorales et postérieure du limbe élargies, à peine relevées, et non creusées en gouttière; dervière la plaque frontale, une petite plaque triangulaire, interposée aux pariétales; pas d'occipilale; trois grandes écailles aux talons; queue longue.

Des deux espèces déjà décrites dans ce genre par Schweigger, qui les a d'abord établies sous les noms d'Emys expansa et d'E. Dumeriliana, c'est à la première que cette nouvelle Podocnémide ressemble le plus, et cependant, malgré l'analogie qu'elles offrent entre elles, relativement à leur conformation générale, il est facile de les distinguer.

La carapace est, en effet, plus déprimée dans l'espèce inédite, surtout chez l'individu adulte rapporté par M. Lewy; elle l'est un peu moins chez le jeune sujet, précédemment indiqué, originaire de la République de Venezuella et envoyé par M. Bauperthuis.

Cette différence, due, selon toute probabilité, à celle de l'âge, est pourtant moins marquée que chez la Podocnémide éla rgie.

Une dissemblance moins évidente dans la largeur des régions brachiale et fémorale de la carapace de la P. DE LEwY; l'absence d'une dépression en gonttière sur la moitié postérieure du limbe; la présence,-derrière la frontale, d'une petite plaque très-allongée dans le jeune âge; et séparant l'une de l'autre, dans une partie de leur étendue, les deux pariétales; une longueur proportionnelle un peu plus considérable du museau; une grande écaille de plus au talon; une queue plus allongée : telles sont les principales particularités qui éloignent forcément l'espèce nouvelle de la P. ÉLARgiE.

Il faut y joindre celles du système de coloration, qui consiste en une teinte, non pas brune mêlée de roussâtre, mais d'un vert olive, plus ou moins vermiculé de noir. Toutes les parties molles ont une nuance verte peu foncée, 
et le sternum n'est pas jaune: il est uniformément verdâtre chez les jeunes sujets, et assez abondamment maculé de noir chez l'adulte.

Les plaques du disque n'ont pas une forme tout à fait semblable, à ce qui se voit, chez la P. ÉLARGIE. La dissemblance la plus considérable résulte d'une moindre hanteur proportionnelle des costales, et toutes les vertébrales, par cela même, sont plus larges, si ce n'est la dernière qui, au contraire, est plus étroite. Le plastron, par suite de la dépression de la carapace, a les ailés moins relevées; ce earactère n'est pas aussi apparent chez le sujet de petite taille, où l'échancrure postérieure de cette pièce osseuse est moins profonde que sur l'autre échantillon : ce sont peut-être des différences de sexe.

Une comparaison avec la P.DE DuMÉrlL serait inutile, car il y a, en quelque sorte, dans l'espèce inédite, exagération des caractères de conformation qui forcent à éloigner l'une de l'autre les P. DE Dumérul et ÉLARgie, seuls représentants, jusqu'alors connus, du genre dont il s'agit.

Le plus grand des deux sujets offre les dimensions suivantes : long. de la carap., $\mathrm{O}^{\mathrm{m}} 26$; larg. au nivenu des bras, $\mathrm{O}^{\mathrm{m}} \mathrm{s} 6$, au niveau des membres post., $0^{\mathrm{m}} 24$; long. de la téte, $\mathrm{o}^{\mathrm{m}} \mathrm{0} 55$, du col, $\mathrm{o}^{\mathrm{m}} \mathrm{0} 5 \mathrm{o}$, de la queue, depuis son origine, jusqu'uu clonque, $0^{\mathrm{m}} 09$, au delà du cloaque, $0^{\mathrm{m}} \mathrm{o} 3$.

Santa Fé de Bogota: M. Lewy, le plus grand individu; et le plus petit, de la République de Venezuella, par M. Bauperthuis.

GEXTE PENTONYX,PMTNY. DUM., BIB.

Ce petit groupe d'Élodites Pleurodères se distingue très-facilement de tous les autres Chéloniens de la mème sous-famille, par la présence de cinq ongles à chaque patte. Il ne pourrait être confondu, à cause de ce caractère, qu'avec le genre Stmrothère où la mobilité antérieure du plastron établit une différence tout à fait tranchée.

Les auteurs de l'Erpét. génér. ont décrit deux Pentony :

Le premier, le P. DU CAP, constitue une espèce parfaitement caractérisée à laquelle ils ont rapporté IA Roussatre de Lacépède et l'Einys olivacea de Schweigger, fondées par ces deux naturalistes sur l'examen de deux carapaces, l'une due à Sonnerat et l'autre à Adanson, et que le Muséum conserve comme TYPES. 
Le second PENT. (P. D'A DÁsson) était représenté dans les Collections par une seule carapace sans plastron, recueillie par Adanson au cap Vert, et qui, après avoir successivement servi de TYPE à l'Emys Adansonii de Schweigger et à l'Hydraspis Adansonii de Gray, avait été considérée, avec beaucoup plus de justesse, par les auteurs de l'Erpét. générale, comme ayant appartenu à une Tortue du genre Pentonyx.

Ces derniers avaient été guidés dans cette détermination presque complétement exacte, malgré l'absence des membres, par l'analogie de conformation de cette boîte osseuse et de celle du P. Do CAP. Il fallait attendre, pour en vérifier l'exactitude, que de nouveaux animaux plus complets pussent être comparés à cette carapace. - Or, cette comparaison a pu être faite dans ces derniers temps, après le retour de la grande expédition au Nil blanc, entreprise par ordre du Pacha d'Égypte, et dont l'habile commandant, M. d'Arnaud, a fait présent au Muséum d'un assez grand nombre d'animaux très-intéressants, recueillis pendant ce voyage.

La similitude parfaite d'Élodites de différents âges, provenant de ce don, et du Type, jusqu'alors unique, du P. D'Adasson de Dum. et Bib., tant pour la conformation générale, que pour le systeme de coloration, ont démontré que ces Élodites appartiennent à la même espèce que ce Type. Seulement une particularité très-intéressante se trouva ainsi révélée: c’est que le plastron, au lieu d'ètre immobile, est composé de deux pièces, dont l'antérieure se meut sur l'autre.

Ca n'est donc plus dans le genre Pentonyx que pouvaient être laissés et la carapace sans plastron, provenant du cap Vert, et les individus, parfaitement identiques, rapportés par M. d'Arnaud. Il est devenu nécessaire de les placer dans le genre suivant, c'est-à-dire dans celui des Sтеrnotнìres, qui joint à une assez grande analogie de formes avec le précédent, et à la présence de 5 ongles à chaque patte, la mobilité de la partie antérieure du plastron.

Le Pent. D'Adanson est donc, par cela même, rayé du cadre des Élodites, et se trouve remplacé par le Sternothére b'Adanson qui, dans le Calal., est décrit dans ce dernier genre sous le $n^{\circ} 3$ ter.

Le genre Pentonyx n'aurait plus renfermé, dans le Catal., qu'une espèce, (Pent. du Cap), si le Muséum n'avait reçu, dans ces derniers temps, un spé- 
cimen d'une autre espèce recueillie en Abyssinie par M. Rüppel qui l'a nommée P. Gehafie ${ }^{1}$.

\section{XVI. - 2 bis. Pfatonyx Gehafie, P. Gehnfie, Rüppel.}

Rüppel, Neue Wirbelth. su der Faun. von Abyssin. Amphib., p. 2, pl. 1.

Pelomedusa Gehafiæ, Gray, Cat. of. Tortoises, p. 38.

Pentonix Gehafie, Dum. et A. Dum., Catal. méth. des Rept., p. 18, no 2 bis.

Carapace dun brun verdátre, régulièrement ovalaire, assez déprimée, sans gouttière au milieu, et munie d'une très-légère carène; plaques sternales de la troisième paire ne se touchant pas par leur sommet, sur la ligne médiane.

La tète et le cou sont larges et déprimés, relativement aux espèces voisines. La queue est courte et assez grosse. L'échantillon du Muséum est trèsprobablement une femelle, la queue dépassant à peine la carapace, ce qui, au rapport de M. Rüppel, est un signe distinctif de ce sexe, ainsi que l'absence de stries circulaires, coupées par des lignes saillantes qui ne se voient que sur le mâle, et dont aucune trace ne se remarque sur cet échantillon.

Chez l'un et chez l'autre sexe, dit cet observateur, la couleur de la carapace, de la partie supérieure de la tête, du cou et des pattes, est un brun vert; ces dernières, ainsi que le milieu de la tête, portent des taches de mème couleur, mais d'une nuance plus foncée. Toutes les parties inférieures sont d'un jaune d'ocre clair. L'iris est brun, entremêlé de petites lignes fines et sinueuses d'un jaune d'or.

Il parait que cette espèce ne dépasse jamais $o^{\mathrm{m}} 30$. La carapace du spécimen du Muséum a une longueur de $0^{\text {m }} 138$.

"Ces animaux sont très-nombreux dans toutes les eaux courantes et stagnantes, sur les escarpements des Côtes orientales de l'Abyssinie. Ils sont, le plus souvent, cachés dans les trous des rochers, et paraissent craintifs; ils se nourrissent de mollusques, d'insectes aquatiques et de petits poissons dont ces eaux fourmillent. $)$

" A la fin d'octobre, j’ai trouvé dans une femelle dix-sept oufs parfaitement formés; ils étaient longs chacun de 9 lignes, de forme elliptique, et

1. Ce naturaliste s'est servi, comme dénomination spécifique, du mot arabe que les habitants des environs de Massouah (Côte maritime de l'Abyssinie) emploient pour exprimer l'odeur repoussanté que cette Tortue répand autour d'elle. 
d'une couleur jaunâtre sale; leur coque était mince, le vitellus avait une teinte jaune-orangé vif ; l'albumine en était transparente comme de l'eau. » (Rüppel, loc. cit.)

\section{GENAE STERTOTHETE. STERNOTHERUS. BELL.}

Aux trois espèces de ce genre, dont les différences avec le précédent sont bien nettes, comme je l'ai rappelé plus haut, il faut en joindre deux nouvelles.

Les trois qui sont déerites dans l'Erpét. génér. (S. NolR, S. NOlRátre et S. marrox ) ont entre elles une assez grande analogie.

Les caractères énoncés dans la description de chacune d'elles permettent cependant de les distinguer facilement. 11 est également aisé de ne pas les confondre avec les deux espèces inscrites à leur suite sur le Catal., et qui sont les S. sinueux et D'A Daxsox. Le premier est originaire de l'Afrique australe.

\section{XVIT. - 3 bis. Sternothère sinueux, St. sinuatus Smith.}

Smith, Illustr. of the zool. of South. Afr.,pl. I, page sans numéro".

Stehnothère sinueux, $S t$. sinuatus, Dum. et A. Dum., Catal. des Rept., p. 19, $\mathbf{n}^{\circ} 3$ bis.

Carapace d'un brun verdâtve foncé; les trois plaques vertébrales moyennes portant une saillie d'où résulte une carène, et bordées, de chaque côté, au niveau de leur jonction avec les plaques costales, par un sillon assez profond; sternum rectiligne en arrière des ailes; museau court.

La carapace est oblongue, convexe, assez relevée; le bord postérieur du limbe est presque horizontal et dentelé. Le plastron est entier en avant où il est mobile (caractère générique), et profondément échancré à son extrémité caudale. La tête est large et déprimée, le musean court et arrondi, la mâchoire supérieure échancrée à sa région moyenne pour recevoir un petit prolongement médian de l'inférieure. Il y a deux petits barbillons sous le menton. Les doigts sont courts et armés d'ongles robustes.

1. M. Gray (Cat. of Tort., p. 37) regarde ce St. "comme identique au St. marron, car il range la nouvelle dénomination donnée par M. Smith, parmi les synonymes de ce dernier dont cependant il diffère spécifiquement.

Ce naturaliste décrit une autre espèce, qu'il nomme St. Derbianus (Lord Derby's St.), originaire du Port-Natal. Il en donne une diagnose qui ne peut pas se rapporter à l'espèce de M. Smith. Le Musée de Paris ne possède pas cette espèce. 
La carapace est, en dessus, d'un brun verdâtre foncé, plus sombre encore vers le limbe; le plastron a une teinte orangée pàle, nuancée d'un brun rougeâtre profond, surtout abondant à son pourtour. La tète est, en dessus, d'un jaune clair, finement marbré de lignes brunes, et brunâtre dans le reste de son étendue.

Celte Élodite atteint une assez grande taille. Le plus grand spécimen de la Collection offre les dimensions suivantes : longueur de la carapace $o^{m} 33$, du plastron $o^{m} 3 o$; largeur di disque en avant $o^{m} 2 x$, en arrière $o^{m} 24$; longueur de la tête $0^{\mathrm{m}} 06$, du cou $\mathrm{o}^{\mathrm{m}} \mathrm{o} 8$, de la queue $\mathrm{o}^{\mathrm{m}} \mathrm{o5}$.

La conformation remarquable du test distingue si nettement ce $\mathrm{Sr}$. de toutes les espèces rapportées à ce genre, qu'une énumération des autres caractères, propres à établir les différences qui les séparent, serait inutile.

Les échantillons du Muséum viennent de l'Afrique australe : les uns sont dus à M. J. Verreaux, les autres à M. Delgorgue qui avait exploré avec un grand succès la Còte de Natal. Au reste, d'après les indications de M. Smith, c'est entre les $24^{\mathrm{e}}$ et $25^{\mathrm{e}}$ degrés de latitude que cette Tortue est le plus abondante: ce qui tient sans doute, selon ce naturaliste, à ce que recherchant les retraites les plus sûres, elle trouve dans cette zône, plus qu'ailleurs, des étangs d'une grande profondeur. Aussi est-il difficile de se la procurer.

XViti. - 3 ter. Sternothère d'Adanson, St. Adansonii, Dum. et A. Dum.

Emys Adans., Schweigger, Prodr. Arch. Königsb., t. I, p. 308, spec. 27.

Hydraspis Adans., Gray, Synops. Rept., p. 40, spec. 2.

Pentonyx Adans., Dum., Bib., Erpét. génér., t. II, p. 394.

Pelomedusa? Adans., Gray, Cat. of Tort., p. 38.

Stenvothère d'Adarson, St. Adans., Dum. et A. Dum., Catal. des Rept. p. 19, no 3 ter.

Carapace ovoũde, fauve, piquetée de brun; dos fortement caréné '; plastron mobile en avant, ¿un jaune plus ou moins nuancé de brun rougeátre; tete plate, ussez large, de la mème couleur que la carapace, couverte de fines vermiculations d'un brun jaunàtre, analogues à celles du lest; cinq ongles en avant et en arriere; queue courte.

Ces caractères, joints à la description de la carapace donnée dans l'Erpél. génér., suffisent pour bien faire reconnaître cette jolie espèce, qui se distingue

1. Cette première partie de la diagnose est celle du Pentonyx d'Adunson dans l'Erpét. génér.

La seconde partie contient les caractères qui étaient restés ignorés, parce qu'on ne possédaif pas de sujets entiers. 


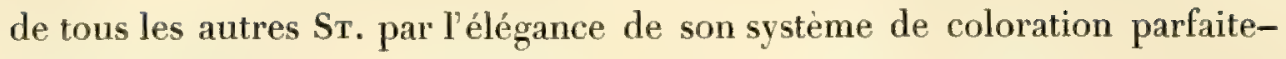
ment identique sur les individus de différents âges renfermés dans la Collection.

C'est, comme je l'ai déjà dit, dans les régions de l'Afrique parcourues pour suivre le cours du Nil blanc, que ces Élodites ont été prises par M. d'Arnaud.

Les dimensions du plus grand spécimen sont les suivantes : Longueur de la carapace, $o^{\mathrm{m}} 160$, du plastron, $\mathrm{o}^{\mathrm{m}} 140$; largeur du disque en avant, $\mathrm{o}^{\mathrm{m}} \mathrm{1}_{\mathrm{o}}$, en arrière, $o^{m} 12$; long. de la tête, $o^{m} \mathrm{o} 35$, du cou, $o^{\mathrm{m}} \mathrm{o}$ qo, de la queue, $\mathrm{o}^{\mathrm{m}} \mathrm{o} 25$.

\section{GENEE PUATEMTWE. PEATEUTS. WAGLER.}

Les Élodites pleurodères comprises dans ce groupe se reconnaissent à l'aplatissement du disque et de la tête, qui est couverte, soit d'une seule lame écailleuse, soit de petites plaques; à l'immobilité du sternum et aux cinq ongles dont les pattes de devant sont armées, tandis que les postérieures n'en ont que quatre.

Ce genre est nombreux en espèces : il en comprend treize qui ont été décrites, dans l'Erpét. génér., d'après des individus que renferme la Collection, si ce n'est deux d'entre elles, l'une la PL. DE Neuwied connue seulement par l'ouvrage du Prince Maximilien de Neuwied qui l'a figurée sous le nom d'Emys depressa, et l'autre la PL. a Pievs rouges, par les détails et les dessins dus à Spix et à Wagler.

Il n'y aurait aucune particularité nouvelle à ajouter à ce genre, si le Muséum n'avait récemment acquis une Élodite de la Nouvelle-Hollande qui, bien 'qu'elle offre tous les caractères de la Platémyde décrite sous le nom de Pl. de Macquarie, présente cependant quelques différences dignes de remarque. Elles ne semblent pas suffisantes pour motiver l'établissement d'une espèce nouvelle, d'autant plus qu'elles peuvent dépendre du sexe. Le nouveau spécimen a été vendu déjà monté, et comme étant une femelle, et l'on ignore le sexe de l'échantillon, jusqu'alors unique, depuis longtemps conservé dans les Collections où il a servi de TYPE à Cuvier pour son Emys Macquaria, (R.anim., $2^{\mathrm{e}}$ éd , t. II, p. Ir, note 2.)

Il est toutefois utile de signaler les petites dissemblances de ces deux Tortues. On pourra ainsi, par la comparaison ultérieure de nouveaux individus, à l'aide de ces documents, décider si, dans ce genre, dont douze des espèces 
ORDRE DES TORTUES. - C ELODITES, POTAMITES ET THALASSTES. 249

connues appartiennent à l'Amérique du Sud, il y en a une seule originaire de l'Australie, on bien, au contraire, si dans ce vaste Continent, il en existe deux bien distinctes ${ }^{\mathrm{T}}$.

On peut, d'ailleurs, supposer que certaines différences entre ces deux Platémydes sont purement individuelles. C'est peut-ètre par un motif semblable que les détails donnés par M. Gray (Cat. of Tort., p. 42), sur l'espèce dont il s'agit, et qu'il nomme Chelymys Macquaria, ne sont pas tout à fait conformes à ceux de l'Erpél. génér.

Quoi qu'il en soit, le nouveau spécimen, dont je fais ici mention, se distingue de l'ancien, par un volume proportionnel plus considérable de la tête; par un aplatissement plus prononcé du disque; par une largeur moindre de la région postérieure de la carapace comparée à l'antérieure. En outre, la plaque nuchale manque presque complétement: elle ne consiste qu'en un petit rudiment linéaire. La queue est moins longue; il existe un écartement un peu plus marqué des plaques du limbe en arrière, d'où résultent, dans cette région, de petites dentelures indiquées par M. Gray, et qui ne se voient pas sur l'ancien type. La conleur de ce dernier, enfin, est un brun plus clair que chez l'animal qui lui est comparé.

\section{ET IV. - TORTUES FIUUIATILES ET MARINES OU POTAMITES ET THALASSITES.}

Ces deux familles n'ont subi aucune modification dans le nombre de leurs espèces. Le Calalogue contient l'indication de tous les échantillons nouveaux reçus depuis la publication du t. II de l'Erpét. génér., et tous sont rentrés sans difficulté dans les divisions établies.

1. Beaucoup de questions de géographie zoologique n'auront point de solution, tant qu'on ne connaîtra pas mieux la faune si remarquable de la Nouvelle-Hollande. Il doit y rester, en effet, bien des découvertes ả faire, malgré les belles eĺ nombreuses collections qu'y ont formées déjà les Anglais, et parmi nos compatriotes, MM. Quoy et Gaimard, Lesson et Garnot, M. Arnoux, et spécialement M. J. Verreaux, dont les envois successifs, pendant son séjour en Australie, ont tant enrichi le Muséum' d'animaux nouveaux et les plus précieux, comme on le verra par la suite de ce travail. 


\section{ORDRE DES SAURIENS.}

Cet ordre, si nombreux en grandes familles, renfermant une quantité -considérable de genres et d'espèces, est, par cela même, assez difticile à définir.

Les caractères suivants donnent cependant une notion assezexacte, quoique générale, des Sauriens, en les isolant nettement des Chéloniens, des Ophidiens et des Batraciens : Corps sans carapace, le plus souvent écailleux, et avec des membres; des côtes et un sternum; des paupières, un canal auditif externe; mâchoires dentées; branches du maxillaire inférieur soudées entre elles.

Les différences remarquables qui existent dans l'organisation interne, mais surtout dans les caractères extérieurs du grand nombre de Reptiles auxquels convient cette diagnose, ont nécessité de larges subdivisions, soumises elles-mêmes à des coupes secondaires, très-multipliées. C'est ainsi que le point de départ de la classification a été le partage de tous les Sauriens en huit familles, dont les noms sont empruntés aux genres qui peuvent être considérés comme types de chacune d'elles. Ces familles, en les énumérant selon l'ordre méthodique, sont celles des Crocodiliens, des Caméléoniens, des Geckotiens, des Varaniens, des Iguaniens, des Lacertiens, des Chalcidiens et des Scincoidiens.

Il serait sans utilité de rappeler ici les dissemblances frappantes qui éloignent les unes des autres ces familles, toutes organisées d'après un plan général commun, mais plus ou moins profondément modifié dans chaque famille.

La première et la deuxième, les seules qui doivent nous occuper en ce moment, se sont enrichies de quelques représentants noureaux. 
PREMIERE FAMILLE : CROCODILIENS OU ASPIDIOTES.

La dénomination, empruntée du grec, par laquelle on désigne cette famille de Sauriens, et qui rappelle l'armure d'écussons solides èt souvent osseux de leurs téguments, indique, tout d'abord, l'une des particularités les plus remarquables de leur organisation. Si, à ce caractère essentiel, on joint les suivants, on a une notion des traits principaux et essentiels de leur structure: corps déprimé, allongé; queue plus longue que le tronc, comprimée latéralement et garnie de crétes en dessus; quatre pattes courles, dont les postérieures ont les doigts réunis par une membrane natatoire; trois ongles seulement à chaque patte; téte déprimée, allongée en un museau au devant duquel se voient des narines rapprochées sur un tubercule charnu, garni de soupapes mobiles; bouche fendue au delà du crâne; langue charnue, adhérente, entière, non protractile; dents coniques, simples, creuses à la base, inégales en longueur, sur un seul rang; organe génilul mâle, simple, sortant par un cloague longitudinal.

Ainsi définis et groupés, les Crocodiliens forment une famille très-naturelle, dont les trois divisions qui la composent ont entre elles une si frappante analogie, que, dans l'E'pét. génér., elles ont été considérées par mon père et par Bibron, comme ne devant constituer que des sous-genres dans le grand genre Crocodile.

Ces trois sous-genrés sont, d'ailleurs, parfaitement distincts entre eux par la conformation du museau.

Ainsi dans les Gavials, il est long, très-étroit, presque arrondi, dilaté à l'extrémité.

Quant aux sous-genres Caïman et Crocodile, où il est moins long, moins étroit, et sans renflement terminal, la position occupée par les quatrièmes dents inférieures, lorsque la bouche est fermée, ne permet, entre eux, aucune confusion. Tandis, en effet, que dans les Caimans ces dents se logent et se cachent dans des fosses creusées, dans ce but, de chaque côté de la mâchoire supérieure; elles restent apparentes, au contraire, dans les Crocodiles, où ces fosses sont remplacées par des échancrures profondes, latérales, destinées à servir de passage à ces dents.

Ces particularités ne sont pas les seules qui éloignent l'un de l'autre ces 
trois sous-genres, et l'on trouve, dans l'Erpét. génér., tous les détails qui se rapportent à ce sujet.

Je n'ai voulu rappeler ici que les traits les plus frappants des trois coupes établies dans le groupe des Crocodiliens.

Des deux premieres, la seconde seule, qui comprend les Crocodiles proprement dits, a subi quelques changements par l'adjonction d'espèces qui n'étaient pas connues en 1836 , époque où parut le troisième volume de l'ouvrage de mon père et de Bibron, lequel est consacré à l'histoire des quatre premières familles de l'Ordre des Sauriens ${ }^{\mathrm{r}}$.

L'un de ces Crocodiles appartient aux espèces dont le museau allongé établit une sorte de passage entre ce sous-genre et celui des Gavials. La première description qui en ait été donnée est due à Bennett. On la trouve insérée dans les Proceed. of the zool. Soc. pour l'année i 835, mais elle n'était pas connue en France avant l'impression du tome III, car ce curieux animal n’y est pas signalé.

XIX. - 7 bis. Crocodıle BeC-ÉTroIT, C. leptorhyncus. Bennett.

Bennett, Proc. of the zool. soc., 1835, p. 128.

Bennet's false Garial", Mecistops Bennettii, Gray, Cat. of. Tort. Croc., etc., p. b7.

C. BeC-ÉTrort, Leptorhyncus, Dum. et A. Dum., Catal. des Rept., p. 29, nº 7 bis.

Museau allongé, formant une sorte de bec; largeur de la téte égale seulement au tiers de sa lonyuєur; deux petites plaques nuchales ovales, suivies d'un vang dé quatre autres plus petites; trois paires de plaques cerviales constituant comme un bouclier, et dont les deux premières paires sont formées par des plaques plus grandes que celles du dernier rang.

La description peut être complétée par l'énumération des caractères suivants.

1. Je rappelle ici, mais sans y insister, parce que les descriptions de l'Érpét. génér. sont suffisantes pour établir le fait, que les $C_{\mathbf{R}}$. décrits dans cet ouvrage, comme Variétés $B$. et $C$. du CR. vulgaire peuvent ètre considérés comme appartenant aux espèces nommées, l'une $C_{R}$. des marais, $C r$. palustris, par Lesson, et l'autre, Cr. Margivaire, Cr. marginatus, par Geoffroy-Saint-Hilaire.

Peut-être en est-il de même pour la Variété $D$ de l'Erpét. génér., laquelle a pour type le CR. vert d'Adanson, devenu plus tard le type du Cr. saché de Geoffroy.

2. M. Gray emploie, de préférence, ce mot, comme étant plus conforme au nom indien que celui de Gavial introduit dans notre langue par M. de Lacépède.

3. Sur l'exemplaire TYPE de Bennett, ce second rang manque, et c'est pour ce motif qu'il n'en est 
Sur le dos, il y a dix-huit bandes d'écussons, dont les deux premières ne sont constituées que par quatre plaques, comme les trois dernières, et dont toutes les autres ont six pièces. On compte trente-deux anneaux à la queue, où les plaques médianes s'effacent à partir du dixième jusqu'au dix-huitième au niveau duquel la crête devient unique. La mâchoire supérieure porte trente-quatre dents, et l'inférieure trente. La teinte générale est un brun fauve relevé par de petits dessins noirs sur les plaques des parties postérieures, et par de grandes taches, de la même nuance, sur la queue.

L'analogie entre ce $C_{R}$ 。 et le $C_{R}$. a nuque curasséE est évidemment trèsgrande, puisque Bennett, ainsi qu'il le dit dans sa note, a longtemps considéré l'individu qui lui a servi de Type comme appartenant à cette dernière espèce. Après la mort de l'animal qu'on avait conservé vivant dans les jardins de la Société zoologique, ce naturaliste a pu s'assurer, par un examen attentif, des différences suivantes : La tète, bien que très-allongée déjà dans le $C_{R}$. à nuque cuirassée, l'est davantage dans le Leptorhynque; le rapport, en effet, de la largeur à la longueur n'est plus ici de 1 à 2 I/2, mais de 1 à 3 .

Le $C_{R}$. A nuque currassée adulte nous étant inconnu, nous conservons quelques doutes sur la distinction établie entre ces deux espèces, et M. Gray (Cat. of. Tort. Croc., etc.) dit, en parlant du Cr. a nuQ: e Currassée, qu'il n'est probablement qu'une variété du CR. Leptorhynquf.

Quant aux rapports que semblerait établir entre le $\mathrm{Cr}$. bec-étroit et le Gavial, le remarquable allongement du museau, ils ne sont pas tellement frappants, en ne tenant mème pas compte des autres caractères, qu'on ne puisse facilement, et presque à la première vue, saisir les dissemblances. Ainsi, dans ce $\mathrm{Cr}$., comme dans celui de Journu dont la tète présente une assez grande analogie de conformation avec celle de ce dernier, le bec offre, jusqu'à un certain point, la forme d'un cône, qui, par sa base se confond avec

pas fait mention dans la diagnose de notre Catal. L'auteur fait observer qu'il résulte de son absence une différence avec le $\mathrm{Cr}$. à nuque cuirassée, où ce rang existe, comme l'indique la figure donnée par Cuvier (Ossem. fossiles, t. V, 2e part., pl.v, fig. 1-2) Si, par cette particularité, notre exemplaire se rapproche un peu de l'animal qui a servi de type à Cuvier pour cette dernière espèce, il en diffère, de la facon la plus notable, par le nombre des rangs de plaques cervicales : il y en a trois rangs seulement, chez le Cr. вес-Étrorr du Musée de Paris, et quatre sur le Type de Bennett, comme l'indique la diagnose du Catal, tandis qưon en compte cinq sur le dessin qui vient d'être cité, où les deux premiers sont formés chacun de deux plaques volumineuses et les trois autres, de pièces beaucoup plus petites : de ces trois derniers, un. seul se voit dans le Cr. bec-étroit de notre Musée. 
le crâne, au lieu d'être, comme dans le Gavial, une partie presque distincte du crâne, qui, dans ce dernier genre, est court, et représente un hexaèdre déprimé, plus élargi en arrière qu'en avant.

L'échantillon unique du Muséum a été donné par le Musée Britannique. Or, celui que cet établissement possède encore provient de l'Afrique occidentale.

Il y a donc lieu d'attribuer la même origine à celui du Musée de Paris.

Ses dimensions sont les suivantes :

Long. de la tête, $o^{\mathrm{m}} 3 \mathrm{I}$, du tronc, $\mathrm{o}^{\mathrm{m}} 52$, de la queue, $\mathrm{o}^{\mathrm{m}} 74$; largeur de la tête, en arrière, $\mathrm{o}^{\mathrm{m}} \mathrm{I} \mathrm{I}$, et de l'extrémité antérieure du museau, $\mathrm{o}^{\mathrm{m}} \mathrm{or} .7$.

Parmi les Crocodiliens intermédiaires, en quelque sorte, aux Gavials et aux Crocodiles proprement dits, il faut placer une espèce nouvelle que le Muséum ne possède pas, mais qui est conservée au Musée de Leyde où elle a été reçue de l'A rchipel Indien. Elle est connue en France, par la description et la figure qu'en a données M. Sal. Müller, dans le recueil précédemment cité, et concernant la zoologie des Possessions Néerlandaises aux Indes orientales.

M. Gray (Cat. of Tort. Croc., etc., p. 58) rapproche cet animal du Cr. de Јоити. Il est impossible, sans avoír sous les yeux les objets mêmes sur lesquels la comparaison doit porter, d'émettre une opinion relative à la place qu'il convient d'assigner méthodiquement à ce nouveau Crocodilien. Il semble cependant, d'après la belle représentation que renferme l'ouvrage hollandais, qu'il doit appartenir à une espèce distincte.

Le Cr. De jourvo si remarquable, comme les précédents, par l'extrême allongement du museau, avait été décrit, dans l'Erpét. génér., d'après un individu unique du Musée de Bordeaux. Depuis cette époque, les Collections de Paris ont reçu du Musée de Marseille, un très-beau représentant de cette curieuse espèce.

Elles se sont, en outre, enrichies d'une espèce nouvelle de l'Amérique centrale, assez voisine du CR. a Museav AIGU, et dont M. Morelet a recueilli deux individus dans le Lac Flores (Yucatan).

La détermination et la description ont éié faites d'après ces deux beaux échantillons, l'un adulte, et l'autre d'âge moyen. Ce dernier' a été récemment déposé dans le Musée de Dijon, et le plus grand des deux a pris place dans les galeries du Muséum, par suite du don qu'en a fait le généreux voyageur à qui nous avons dédié cette espèce. 
XX. -5 bis. Croconile de Morelet, C'r. Moreletii, A. Dum. espìce nouvelle. (Planche $\mathrm{xx}$ ).

Chocodile de Monelet, Cr. Moreletii, A. Dum., Catal. des Rept., p. 28, $\mathrm{n}^{\circ}$ כ bis.

Museau assez effilé; quatre écussons sur la nuque, six sur le cou, disposés en deux rangées transversales, la première, de quatre, et la postérieure, de deux; carènes dorsales de hauteur à peu près égale sur les rangs médians et sur les rangs latéraux; écailles des flancs et des nembres lout à fait lisses, et dépourvues de tubercules; palmure des trois doigts externes des pattes postérieures peu éteridue.

I.e chanfrein est légèrement bombé. Sur le front, deux carènes forment une figure assez analogue à celle qui se voit chez le Cr. rhombifère. l.es carènes dorsales constituent seize bandes transversales, dont la première et les six dernières sont composées de quatre plaques, et les autres de six ou de quatre. La crète caudale est haute. Les écailles du cou, des flancs et des membres sont plates, sans tubercules, ni carènes. Jusqu'à son cinquième cercle écailleux seulement, le dessus de la queue offre quatre rangs longitudinaux d'arètes, dont les médians diminuent rapidement d'élévation; à partir de ce point, jusqu'au dix-huitième cercle, il n'y a plus que les deux rangs latéraux; au delà, on n'en compte plus qu'un. Cette arête caudale qui est donc d'abord quadruple, puis double, et enfin simple, augmente notablement de hauteur, à mesure qu'elle s'éloigne du tronc, ainsi que cela se voit chez la plupart des Crocodiliens. Sur les flancs, on voit quelques écailles saillantes, mais moins nombreuses que chez le Cr. rhombifère, et ne formant pas, comme chez ce dernier, deux lignes parallèles, à droite et à gauche du bouclier dorsal. Les doigts antérieurs sont libres, à l'exception du deuxième et du troisième, qui sont réunis par une petite membrane.

La teinte générale est un brun noirâtre, avec quelques vermiculations d'un brun verdâtre. Le dessous est un gris tirant un peu sur le vert.

Les caractères qui distinguent nettement le Crocodile dé Morelet du Crocodile à museau aigu, sont : les différences dans la forme de la tête, qui, quoique proportionnellement plus courte, a cependant plus de largeur ';

1. Les chiffres suivants mettent ce fait hors de doute: la longueur totale du Cr. de Mor. est de $2 \mathrm{~m} 60$, et la tête, jusqu'à l'angle de la máchoire, a $0^{\mathrm{m}} \mathbf{4 0}$, tandis que la tête du plus grand $\mathrm{Cr}$. à mu- 
la hauteur presque égale des carènes dorsales sur les rangs externes et sur les rangs médians, et le défaut de bosselures ou de carènes sur les écailles de la face externe des membres.

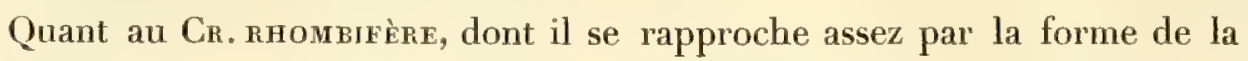
tête et des lignes saillantes du crâne, et quoique moins trapu, par l'ensemble de sa conformation, il s'en distingue par la disposition des rangs d'écailles du bouclier dorsal, par l'élévation de la crète caudale, et surtout par l'absence complète de tubercules ou de carènes sur les écailles de la peau des flancs et des membres.

Lia Flores (Yucatan) : M. Morelet.

D'après ce qui précède, il est évident que ce Cr. de Morelet appartient à une espèce parfaitement distincte des autres Cr. Américains, et en particulier, de celui à museau effilé.

Il ne paraît pas en être de même pour un individu, originaire du Mexique, donné par M. Montluc, Consul de France. S'il n'existait pas chez ce Cr. des différences dans le nombre des pièces osseuses de la région supérieure du cou, il n'y aurait aucun motif d'en faire une mention spéciale. Aussi, ne peut-on, en raison de cette particularité, le considérer que comme le représentant d'une variété qu'on peut définir ainsi :

Croconile a museau effilé, $C r$. acutus, Geoffroy.

Variété à dix écussons cervicaux. Dum. et A. Dum., Cat. des Rept., p. 28.

Plaques composant le bouclier cervical disposées sur quatre rangs, le premier formé de deux plaques, le deuxième de quatre, et les deux derniers de deux.

Peut-être, plus tard, en raison de ce nombre plus considérable des écussons osseux, dont il n'y a que six dans le Cr. à museau effilé, sera-t-on fondé à établir une espèce nouvelle, quand la comparaison pourra porter, non plus sur un seul individu, mais sur plusieurs.

sean effilé, presque égale à la précédente, mesure $0^{\mathrm{m}} 38$, quoique la longueur totale, qui est de $2^{\mathrm{m}} \mathbf{2 0}$, soit inférieure de $0^{m} 40$ à celle de l'autre animal. Quant à la largeur proportionnelle de la tète, tout à fait en arrière, elle est moindre chez le Cr. à museau effilé, car elle est à la longueur de la tète, dans le rapport de 16 à 38 : elle est, au contraire, dans le rapport, beaucoup moins éloigné, de 23 à 40 , chez le Crocodile de Morelet. 


\section{FAMILLE DES GAIÉLÉONIENS OU CHÉLOPODES.}

Le second nom imposé par les auteurs de l'Erpèt. génér. aux animaux groupés dans cette famille est destiné à rappeler une des particularités les plus singulières de leur organisation, qui diffère, sous tant de rapports, de celle des autres Sauriens. Ils ont, en effet, voulu désigner par cette dénomination empruntée à la langue grecque, et qui signifie pieds en pince, la disposition tout à fait exceptionnelle des doigts qui sont réunis entre eux jusqu'aux ongles, en deux paquets inégaux, à chaque patte, trois d'un côté, deux de l'autre.

L'analogie extrême qui existe dans l'ensemble de l'organisation et de la conformation extérieure de ces Reptiles si bizarres, ne permet pas d'admettre plus d'un genre; mais ce genre, qui doit ètre considéré comme le représentant d'une famille spéciale, parfaitement distincte, renferme un assez grand nombre d'espèces. On en connaissait quatorze en r 836. En 185r, nous en avons signalé dix-sept dans le Catalogue, et nous en avons laissé de côté six autres que le Muséum ne possède pas.

Ces dix-sept espèces, qui diffèrent entre elles d'une façon trèsnotable, pourraient ètre rangées en deux groupes.

Le premier comprendrait lés $\mathrm{C}$. à casque triangulaire, plus ou moins pointu et plus ou moins relevé, c'est-à-dire les sept premières espèces : (C. à cape, ordinaire, verruqueux, Namaquois, tigre, nasu, nain). Chez les quatre premiers, le casque est trèssaillant en arrière; il l'est beaucoup moins chez les trois derniers qui, par le peu d'élévation de leur carène occipitale et par sa direction presque horizontale, établissent, en quelque sorte, une transition entre ce groupe et le suivant.

A celui-ci, il faut rapporter les onze autres espèces dont le crâne est à peu près plane, et tout à fait arrondi à sa région postérieure, et non plus triangulaire. Ce sont les C. du Sénégal, panthère, à bandes 
latérales, à baudrier, bilobé, à capuchon, à nez fourchu, de Brookes, de Parson, à trois cornes. Ces quatre derniers, ainsi que le C. nasu, diffèrent de tous les autres par les prolongements qu'ils portent à la région antérieure de la tête, et qui leur donnent un aspect étrange.

Pour le $C$. de Parson, l'on sait maintenant que ces crêtes osseuses sont l'apanage exclusif du màle; mais pour les autres espèces, dont les échantillons sont, en général, fort rares dans les Collections, on ignore s'il en est de même. Quoiqu'il en soit, il est difficile de s'expliquer physiologiquement le but de ces appendices, ainsi que celui de la carène postérieure du crâne.

Les Caméléons, au reste, ne sont pas les seuls Reptiles qui portent ainsi des prolongements. Parmi les Lézards Iguaniens, on trouve le Cératophore, dont le museau est surmonté d'une sorte de corne, l'Arpéphore a trols bandes, $A$. tricinctus $A$. Dum., que j'ai décritet fait figurer (Revue et mag. de Zool., mai i 85 I , $\mathbf{n}^{0} 5$.), et caractérisé par une lame membraneuse, comprimée, mince, que supporte le nez et qui est en forme de sabre ou de faux à deux tranchants, dont le supérieur est légèrement concave et l'inférieur convexe. On peut rapprocher de ces Sauriens le Céraste ÉGYPTIEn, la Vipère hexacanthe, l'ER PÉton décrit d'abord par Lacépède, d'après l'exemplaire, unique jusqu'à ce jour, du Musée de Paris; le Langaha ou Serpent a crête de coq et le Crapaud cornu.

Rien de semblable à ce qui se voit chez les animaux que je viens de citer n'existe chez les trois Caméléons nouveaux du Musée de Paris. Deux de ceux-ci, les C. Namaquors et a CAPE, ont le casque long et pointu; il l'est surtout dans la deuxième espèce; la troisième, au contraire (C. A BAUdrier), ne porte aucune trace de carène sur le crâne, qui est arrondi en arrière.

De ces trois espèces, l'une avait été déjà décrite par M. A. Smith, les autres étaient inédites. 
XXI. - 3 bis. Caméléon Namaquors, Ch. Namaquensis. Smith.

Smith, South. Afr. quarterly journ. $n^{\circ} 5$, p. 17, et lllustr. of the zool of $S$. Afr., Append., p. 3. Chamaleo tuberculiferus, Gray, Cat. of Liz, p. 267.

Caméléor Fanaquors, Ch. Namaquensis, Dum. et A. Dum. Catal. des Repl., p. 32, no 3 bis.

Sur le dos, une rangée de grands tubercules granulés, pointus et saillants; ligne nentrale médiane non dentelée; museau court, sans prolongements; casque occipital proéminent, triangulaire, divisé, le long de la ligne médiane, par une quille élevée et comme dentelée, et bordé, de chaque côté, par une ligne proéminente, presque semi-circulaire, et éyalement dentelée.

Les écailles du corps et de la queue sont petites et semblables entre elles, à l'exception de celles de la carène dorsale dont les tubercules sont au nombre de treize sur l'exemplaire unique du Muséum. Il est de la taille du CaméLÉOX ORDINAIRE, et son système de coloration n'offre rien de particulier à noter : la teinte générale est un gris verdâtre, semé, çà et là, de quelques vergetures noires.

M. Smith dit que tous les individus de cette espèce qu'il a vus pendant son séjour au Cap avaient été recueillis dans le pays des petits Namaquois, vers l'embouchure du Gariep ou Rivière Orange. Le spécimen donné au Muséum par ce savant naturaliste provient de la même localité.

XXII. - i bis. CaMéléot a CAPF, Ch. calyptratus, A. Dum. espè̀ce nOUVelle.

(Planche $\times x 1_{*}$ )

Caméléov a Cape, Ch. Calyptratus A. Dum., Catal. des Rept., p. 31, no 1 bis.

Saillie du dos dentelée, ainsi que la ligne médiane du ventre; casque très-relevé, et à carène fort saillante.

Ce dernier caractère, tout à fait remarquable, puisque la longueur du cas. que, dont la carène est curviligne, est égale à celle de la tête, suffirait seul pour éloigner cette espéce de toutes ses congénères. Elle l'emporte donc par le développement de la carène, sur le C. vulgaire, dont il est par cela même facile de lá distinguer. Le même caractère empêche également qu'on ne la confonde avec le C. verruQueux, semblable au C. vulgatre pour la longueur du casque, mais offrant, comme caractère distinctif essentiel, une rangée longitudinale d'écailles circulaires le long de chaque flanc.

Il résulte des grandes dimensions de la carène, que le crâne semble sur- 
monté d'un haut capuchon terminé en pointe : d'où le nom par lequel nous avons désigné ce singulier animal.

Les faces latérales du casque sont couvertes de grandes écailles plates et circulaires ou polyédriques. Sur le reste du corps, il y a, parmi les petits grains des téguments, quelques grains plus volumineux, irrégulièrement dispersés, et beaucoup plus petits que les grandes écailles du C. verruqueux.

Le front est excavé au milieu, et bordé par des crêtes surciliaires assez hautes, qui ne se prolongent pas tout à fait jusqu'à l'extrémité du museau, et ne se réunissent pas.

Le système de coloration est une teinte grisâtre, assez uniforme, sur laquelle se détache quelquefois une bande latérale jaunâtre.

Ce C. atteint presque la taille des plus grandes espèces du genre.

Le Muséum possède trois exemplaires rapportés de la Région du Nil par M. Botta:

XXIII. - 6 bis. Caméléon a baudrier, Ch. balteatus, A. Dum. espèce nouvelle.

(Planche xxiI).

Caméléon a Baudier, Ch. balteatus, A. Dum., Catal. des Rept., p. $32, \mathrm{n}^{\circ} 6$ bis.

Casque plat, sans carène, arrondi en arrière, à bords saillants et continus avec les aretes surciliaires, qui ne sont pas réunies à leur extrémité antérieure, et ne se prolongent pas jusqu'au bout du museau; grains de la peau nombreux, petits et éganx.

Tels sont les caractères par lesquels cette espèce se rapprocherait surtout du C. DU SFÝGAL, si, contrairement à ce qui se voit chez ce dernier, la ligne médiane inférieure n'était dépourvue de dentelures. La région supérieure porte seule une crête à divisions peu profondes, et presque nulles sur lạ queue.

La teinte générale est un gris d'ardoise, élégamment relevé par un jaune pur sur plusieurs régions, d'abord aux angles de la bouche et sous la gorge, à la face interne des membres, puis sur toute la ligne médiane inférieure, depuis le menton jusqu'à l'extrémité de la queue, et enfin sur les flancs, qui portent chacun, de l'épaule à la hanche, une large ligne jaune, semi-lunaire, à concavité supérieure, et simulant une sorte d'écharpe ou de baudrier.

Par cette dernière particularité, cette espèce nouvelle a quelque analogie 
ORDRE DES SAURTENS. - CAMÉLÉONIENS.

avec le C. a bandes iatérales; mais le casque de celui-ci, d'une forme un peu différente, porte une carène légèrement arquée, et cette ligne saillante manque complétement chez le C. A ва IIDFIER, dont la taille est d'ailleurs beaucoup plus grande.

L'échantillon du Muséum est unique; il a été adressé de Madagascar.

Les espèces originaires de l'Afrique anstrale, qui n'existent pas dans les collections de cet établissement, et que M. A. Smith a décrites, mais non figurées dans ses Illustr. of the zool. of S. Afr., appendix, p. 3, sont au nombre de deux.

L'une qu'il avait déjà précédemment fait connaitre ( $S$. Afr' quarterly journ., $n^{\circ} 5$, p. 17,1831 ), a reçu de lui le nom de Chamaleo taenia-bronchus. Elle peut ètre caractérisée ainsi :

Casque occipilal étroit, prolongé, surmonté de trois carènes: une médiane, et deux latérales; créte dorsale formée de petits tubercules courts; menton et gorge garnis d'une frange dentelée, basse; tempes divisées longitudinalement par une ligne également dentelée.

M. Smith n'a jamais vu qu'un seul représentant de cette espèce.

L'autre CArr. qu'il décrit pour la première fois dans ses Illustr., y est désignée sous la dénomination de $C h$. gutturalis.

La diagnose donnée par ce naturaliste montre, comme il le reconnaît lui-mème, que cet animal a beaucoup de ressemblance avec le C. NAIN, également habitant de la pointe méridionale de l'Afrique. Il est donc inutile de rappeler cette diagnose, et il suffit d'indiquer, d'après le zoologiste Anglais, les différences qui éloignent l'une de l'autre ces deux espèces. Ainsi le C. gottural differe du G. Nain par la longueur des appendices qui forment la frange du cou; celle-ci, d'ailleurs, est lisse, sans écailles granuleuses. En outre, la taille du premier est plus considérable.

M. Gray (Cat. of Liz., p. 268) décrit aussi une espèce nouvelle, Africaine comme les deux précédentes, qui ne se trouve pas au Muséum. C'est le $C h$. ventralis, qui paraît à M. Smith n’être qu'une variété du C. NalN.

Un Cam. de Madagascar, inconnu au Musée de Paris, est, en ontre, signalé par M. Gray. Il est très-remarquable par la présence, sur le nez, d'une grande proéminence à bords dentelés: d'où le nom de $C$. rhinoceratus, choisi par ce naturaliste, pour indiquer cette particularité qui, suivant lui, serait peut-être l'apanage spécial du C. verRuQurox mâle. 
M. Stutchburry (Linn. soc. Transact., t. XVII, $3^{\mathrm{e}}$ partie) a figuré et décrit une espèce originaire de Fernando-Po, nommée par lui Ch. cristatus, et dont l'occiput est bordé par des tubercules pointus.

Enfin, M. Martin a signalé (Proceed. of the zool. Soc. 1838, p. 64,) un individu provenant du même pays que le précédent, et qu'il a dédié à Bibron I, mais que M. Gray (Cat. of Liz., p. 269) semble regarder comme étant la femelle du $C$. à trois cornes.

Je me borne à ces courtes citations, les Ch. toenia-bronchus, gutturalis, ventralis, rhinoceratus, cristatus et Bibroni, dont je viens de parler, ne faisant pas partie des Collections du Musée de Paris.

1. Cette dédicace est accompagnée des témoignages d'estime qu'on retrouve chaque fois que ce nom est cité par les naturalistes. 


\section{EXPLICATION DES PLANCHES.}

PLAxche XIV. - 1. Emyde aréolée, Emys areolata, A. Dum., vue en dessus.

V. page 222. 2. Carapace de la mème, vue en dessous.

3. - - - de profil.

Punche XV. - 1. Emyde de Bérard, Emys Berardii, A. Dum., vue en dessus.

$V$. page 231. 2. Carapace de la mème, vue en dessous.

3. - - de profil.

4 et 5 . Màchoires supérieure et inférieure, vies en dedans.

Puavere XVI. - 1. Cinosterne ensanglanté, Cinosternon cruentatum, Dum. et Bibron, vu de $\boldsymbol{V}$. page 238. profil.

2. Idem, vu en dessous.

3. Carapace du Cinosterne scorpioïde, vue de profil.

PLAxche XIII. - 1. Cinosterne bouche-blanche, Cinosternon leucostomum, Dum. et Bibron, vi $\boldsymbol{V}$. page 239. en dessus.

2. Idem, vu en dessous.

3. Idem, vu de profil.

4. Carapace du Cinosterne de Pensylvanie, vue de profil.

PLaxche XIIII. - 1. Podocnémide de Léwy, Podocnemis Lewyana, A. Dum., vue en dessus.

V. page 242. 2. Tète de la mème, vue de profil.

3. et 4 . Tète de Podocnémide de Duméril, vue en dessus et de profil (jeune âge).

PLAvche XIX. - 1. Podocménide de Léwy, Podocnemis Lewyana, A. Dum., vue en dessous.

V. page 242. 2. Carapace de la mème, vue de profil.

PuAche XX. - 1. Crocodile de Morelet, Crocodilus Moreletii, A. Dum.

V. page 255.

Playche XXI. - 1. Caméléon à cape, Chamæleó calyptratus, A. Dum.

V.p. 259-260. 2. Caméléon à baudrier, Chamæleo balteatus, A. Dum.

PLAxche XXII. - Tètes de Caméléons, vues de profil.

V. paye 257. 1. C. vulgaire. - 2. C. verruqueux. - 3. C. tigre. - $3^{b i z}$ C. Namaquois. - 4. C. nasu. - 5. C. nain. - 6. C. à bandes latérales. - 7. C. du Sénégal. - 8. C. bilobé. - 9. C. à capuchon, - 11.1. C. panthère. - 12. C. de Parson, mâle (la femelle ne porte pas de protubérances tuberculeuses à l'extrémité du museau ). -13 . C. à nez fourchu. -14 . C. de Brookes,

1. Le noméro que porte chacune de ces lètes indique l'ordre saivant lequel les Caméléons sont décrits dans l'Erpét. génér. Le dixième (C. a trois cornes) noos érant inconna, le no 10 doit nécessairement manquer sur cette planche. 


\section{INDEX}

DES

\section{GENRES ET ESPÈGES DE REPTILES}

DÉCRITS, FIGURÉS OU INDIQUÉS DANS CE MÉMOIRE.

ORDRE DES CHÉLONIENS.

I. Chersites ou Tontues terrestres.

I. Tortue mi-dentelée, Smith. 213 Pages. Planches. T. de Verreaux, ' Smith... 215

II. T. émydoìde, S. Müller..... 246

II. Elodites ou Tortues paludines. A Cryptodères.

Cistude de Blanding, Holbr. 217

III. Emyde Caspienne, Var. japo-

naise, Schlegel..........219

IV. E.japonaise, Dum. et A.Dum. 220

V. E. aréolée, A. Dum. ESP. N.. 222 XIV.

VI. E. pseudo-géographique, Lesueur. ............... 22.

VII. É.du Cumberland, Holbrook. 226

VIII. E. du Mobile, Holbrook..... 228

IX. $\dot{E}$. labyrinthique, Lesueur.. 229

X. E. hieroglyphique, Holbr... 230

XI. E. de Bérard, A. Dum., E. N. 231 XV.

É. des Florides, Leconte... 232

$\dot{E}$. de Troost, Holbrook.... ibid. E. de lorégon, Harlan..... 233 Tétronyx de Lesson, Dum. B. ibid. Platysterne mégacéphale, Gray.................234

Xil. Emysaure de Temminck, D. 235 Staurotypes tricaréné, Wagler, et musqué, Dum. Bib.. 236

XIII. Cinosterne ensanglanté, D. Bib. ESP. N ........... 238 XVI.

XIV. C. bouche blanche, Dum. Bib. ESP. N............ 239 XVII.

\section{B. Pleuroderes.}

Pages. Planches.

Peltocéphale tracaxa, Dum.

Bib...............2240

Chélyde matamata, Dum. B\$ 24

XV. Podocnémide de Lewy, A. D.

ESP. N..............242 XVIII

et NIX.

XVI. Pentonyx gehafie, Rüpp.... 245

XVII. Sternothere sinueux, Smith. 246

XVIII. S. d'Adanson, Dum. . 244 et 247

Platémyde de Macquarie, Dum., Bib...............248

III et IV. Potamites et Thalassites ou TorTUES fLUViatiles et MARINES, p. 249.

\section{ORDRE DES SAURIENS.}

I. Crocodiliens ou Aspidiotes.

XIX. Crocodile bec-étroit, Bennet. 252

$\mathrm{XX}$. C. de Morelet, A. Dum., E. N. $255 \mathrm{XX}$. C.à museau effilé, Var.à dix écussons cervicaux.......256

II. Caméléoviens ou Chélopodes.

XXI. Caméléon Namaquois, Sm. 259

XXII. $C$. à cape, A. Dun., ESP. N. ibid. XXI.

XXIII. C.abaudrier, A. Dum., E. N. 260 XXII. Chamæleo tænia-bronchus,

Smith ..............261

Ch. ventralis, Gray....... ibid.

Ch. rhinoceratus, Gray... ibid.

Ch. cristatus, Stutchburry.. 262

Ch. Bibronii, Martin..... ibid.

1. Les esnèces nrécédées d’on numéro d'ordre sont décrites dans ce Méntoire, tandis que celles qui ne portent pas de numéru sont seulement indiquées avec plus ou moins de détaills. 


\section{DESCRIPTION}

\section{DES REPTILES}

NOUVEAUX OU IMPARFAITEMENT CONNUS

DE LA COLLECTION DU MUSÉT D'HISTOIRE NATURELLE

E T

\section{REMARQUES SUR LA CLASSIFIGATION}

ET LES CARACTERES DES REPTILES.

\section{DEUXIÈME MÉMOIRE

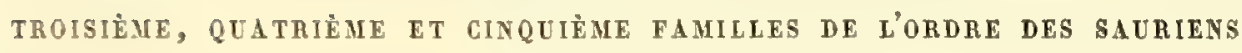 \\ (GECKOTIENS, VARANIENS ET IGUANIENS)}

PAR LE DOCTEUR AUGUSTE DUMÉRIL

AIDE-NATURALISTE AU MUSĹUM,
PROFESSEUR-AGREGE A LA FACULTÉ DE MÉDECINE DE PARIS,

MEMBRE DE LA SOCLE⿱TE⿱ PHILOHATHIQUE,

SECRẺTAIRE DE LA SOCIÉTÉ IMPÉRIALE ZOOLOGIQUE D'ACCLIMATATION.

Deux années environ se sont écoulées depuis l'époque où j'ai publié, dans le tome VI de ce Recueil (p. 209-264, pl. XIV-XXII) le commencement du travail dont ce nouveau Mémoire est une première suite.

J'ai fait connaître alors le but que je me propose, et il suffit maintenant de rappeler que je cherche ainsi à réunir des matériaux propres à former une sorte de supplément à l'Erpétologie générale de mon père et de Bibron.

Anchives du Muséum. T. VIII. ( 1556$)$. 
Je dois à la bienveillance de MM. les professeurs du Muséum, qui en autorisent l'insertion dans lemrs A rchives, de pouvoir, par ce mode de publication, rendre ce supplément beaucoup plus complet et par cela même plus utile, puisqu'il m'est ainsi permis d'y joindre un assez grand nombre de belles figures.

Dans mon premier Mémoire, j'ai passé en revue l'ordre des Chéloniens et les deux premières familles de l'ordre des Sauriens, celles des Crocodiliens et des Caméléoniens. Ce nouveau travail sera consacré à l'examen des trois familles suivantes : les Geckotiens, les Varans et les Iguaniens.

Comme je l'ai fait jusqu'ici, j’exposerai les progrès récents de la science; mais, dans cette révision, je m'attacherai surtout à mentionner les acquisitions nouvelles du Musée de Paris, si riche en espèces rares, et à bien préciser les particularités les plus notables des Reptiles qui, dejà signalés dans le Catalogue ", sont encore peu. connus.

Une semblable étude ne peut pas être entreprise sans que les méthodes employées par les zoologistes pour la détermination des groupes ne deviennent l'objet de l'examen le plus attentif. Il en résulte parfois que l'observateur, se placant à quelque point de vue nouveau ou trop négligé, se trouve amené à présenter des considéralions utiles. Si de semblables occasions me sont offertes, je soumettrai aux naturalistes les remarques auxquelles j'aurai pu être conduit relativement à la classification des Reptiles.

1. Sous le titre de Catalogue méthodique de la collection des Reptiles du Musée d'histois'e naturelle de Paris, j'aì commencé en 48 ă 1 , sous la direction de mon père, la publication d'un releré exact de toutes les richesses de cette immense réunion d'animanx de tous les pays. J'y ai parcouru en entier les deux ordres des Chéloniens et des Sauriens, ainsi qu'une petite partie de l'ordre dos Serpents. Par des circonstances indépendantes de notre volonté, l'impression rle ce travail, dont tous les matériaux sont prêts, nª pas encore pu être continuée. 


\section{ORDRE DES SAURIENS.}

TROISIÈ.UE FAMILLE: GECKOTIENS OU ASGALABOTES.

Parmi les huit familles comprises dans l'ordre des Sauriens et qui doivent être portées à neuf, si l'on tient compte de l'organisation toute spéciale des Amphisbéniens 1, celle des Ascalabotes est l'une des plus naturelles. De mểme que tous les Crocodiliens peuvent être réunis en un seul groupe générique divisé lui-même en trois sous-genres, et que tous les Caméléons, comme les Varans, n'offrent, en quelque sorte, que des modifications spécifiques d'un seul genre, les Geckotiens ont entre eux des analogies assez frappantes, pour qu'ils puissent être considérés comme se rapportant tous, plus ou moins manifestement, à un mème type. Les caractères principaux de ce type consistent : $1^{\circ}$ dans la forme du corps, qui est trapu, déprimé, supporté par des membres courts et robustes, à doigts presque toujours disposés de façon à permettre l'ascension sur les corps les plus lisses, et le plus habituellement terminés par des ongles rétractiles; $2^{\circ}$ dans l'aplatissement de la tête ; les grandes dimensions de la houche, qui contient une langue courte, charnue et libre à son extrémité; le volume des yeux, dont la pupille le plus souvent verticale et frangée dénote des habitudes nocturnes; $3^{\circ}$ enfin, daus l'aspect tout particulier des téguments, qui sont couverts de granulations uniformes ou entremêlées de tubercules plus volumineux.

Néanmoins, les différences dans la conformation des doigts ont été trouvées, avec raison, assez importantes pour nécessiter des coupes secondaires dans cette famille si naturelle.

Sans entrer ici dans aucun détail historique sur les essais successifs des zoologistes pour arriver à une bonne distribution méthodique des Geckotiens, je rappellerai que le mode le plus simple de division est celui qui consiste dans un partage des nombreuses espèces en quatre grands groupes. Le premier comprend tous les Geckos dont les doigts sont nus et non dilatés (Sténodactyliens); le deuxième, ceux qui n’ont les doigts dillatés qu’à

1. J'ai discuté cette question du rang que les Amphisbéniews doivent occuper dans la classe des Reptiles dans un mémoire où j'ai cherché à rassembler toutes les preuves de ce fait que ces singuliers Sauriens apparticnnent à une famille distincte (Revue de zool. Sept. 1852, p. 401). 
leur base (Hémidactyliens); le troisième, ceux à dilatation terminale (Ptyodactyliens ou Sphériodactyliens); le quatrième enfin, les espèces à doigts dilatés dans toute leur longueur (Platydactyliens).

Si je dois nécessairement éviter de revenir sur les divers changements que la classification de ces Reptiles avait subis antérieurement à l'année 1836, époque de la publication du t. III de l'Erpétologie générale, où cette question a été traitée avec les développements suffisants et avec tout le soin qu'elle mérite, il convient, au contraire, de rappeler les tentatives ultérieures.

La première en date dans cette période, est celle de M. Fitzinger qui, dans le premier fascicule de l'oùvrage qu'il a commencé à faire paraître, en $\mathbf{1 8 4 3}$, sous le titre de Systema Reptilium, a modifié la classification des Geckotiens proposée par lui en 1826 .

Il y divise tous les Reptiles soit vivants, soit fossiles, en cinq grandes séries : I. Amblyglossa, Fitz. II. Leploglossa, Fitz. (Wiegm.). III. Testudinata, Oppel. IV. Dipnoa, Leuck. V. Rhizodonta, Fitz. C'est à la première que les Geckos appartiennent en formant un troisième ordre dont le nom (Ascalabota) emprunté à Schneider, est pris ici dans le sens mieux déterminé qui lui a été donné par Wiegmann.

Dans ce nouvel arrangement, le savant naturaliste de Vienne a beaucoup multiplié les genres qu'il a, pour la plupart, subdivisés en sous-genres, en laissant à l'un d'eux le nom générique. Ces coupes secondaires, au nombre de quarante-trois, sont rapportées à vingt-deux genres compris dans quatre familles, celles des Sténodactyliens, des Ptyodactyliens, des Platydactyliens et des Hémidactyliens. La première renferme les Geckos à doigts non dilatés, c'est-à-dire les Sténodactyles et les Gymnodactyles; la deuxième, ceux dont l'extrémité des doigts porte seule un renflement unique ou double : tels sont les Sphériodactyles, les Phyllodactyles et les Ptyodactyles. Les troisième et quatrième enfin, ne sont autre chose que les grands genres Platydactyle et Hémidactyle élevés au rang de familles.

Parmi les vingt-deux genres, il y en a douze établis par divers zoologistes, mais les dix autres, dont sept tout à fait nouveaux, l'ont été par M. Fitzinger.

Dans le but de mettre en relief les caractères distinctifs de ces différents groupes entre eux, j’ai dressé un tableau synoptique pour chacune de ces familles. 


\section{CLASSIFICATION DE M. FITZINGER.}

\section{Fanille I. STÉNODAGTYLIENS.}

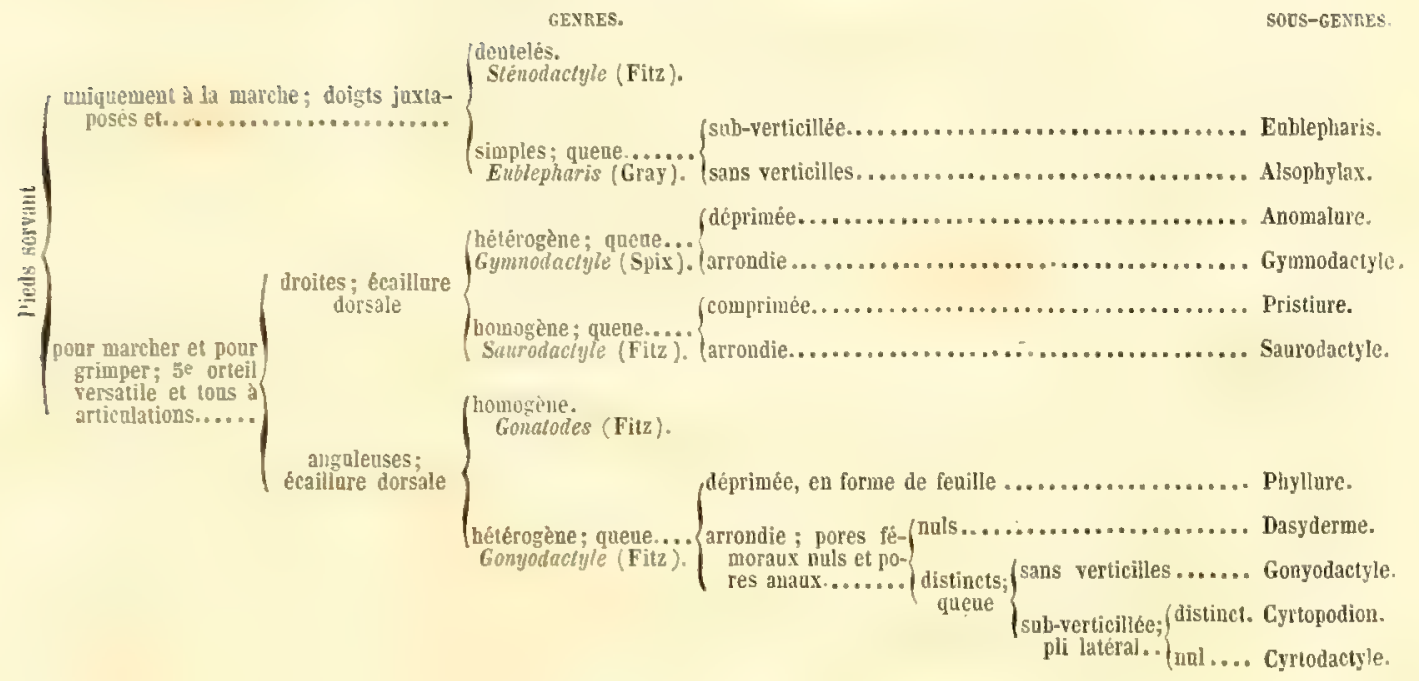

Famile II. PTYODACTYLIENS.

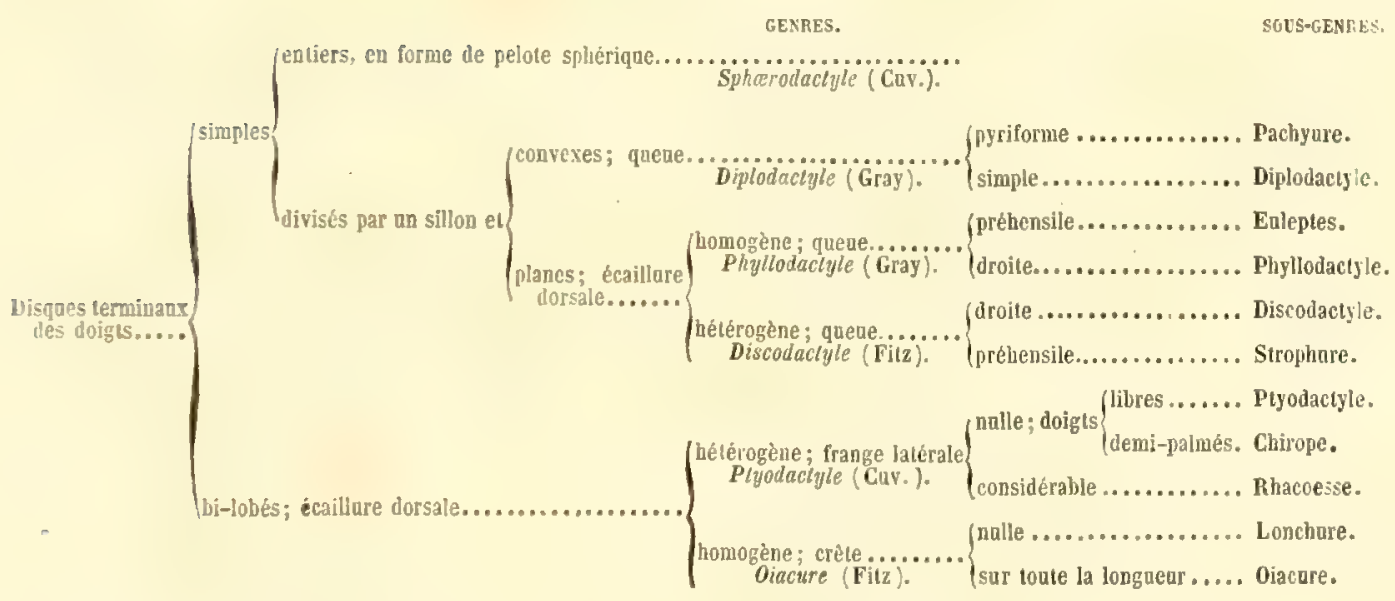




\section{Famile III. PLATYDACTYlieNs.}

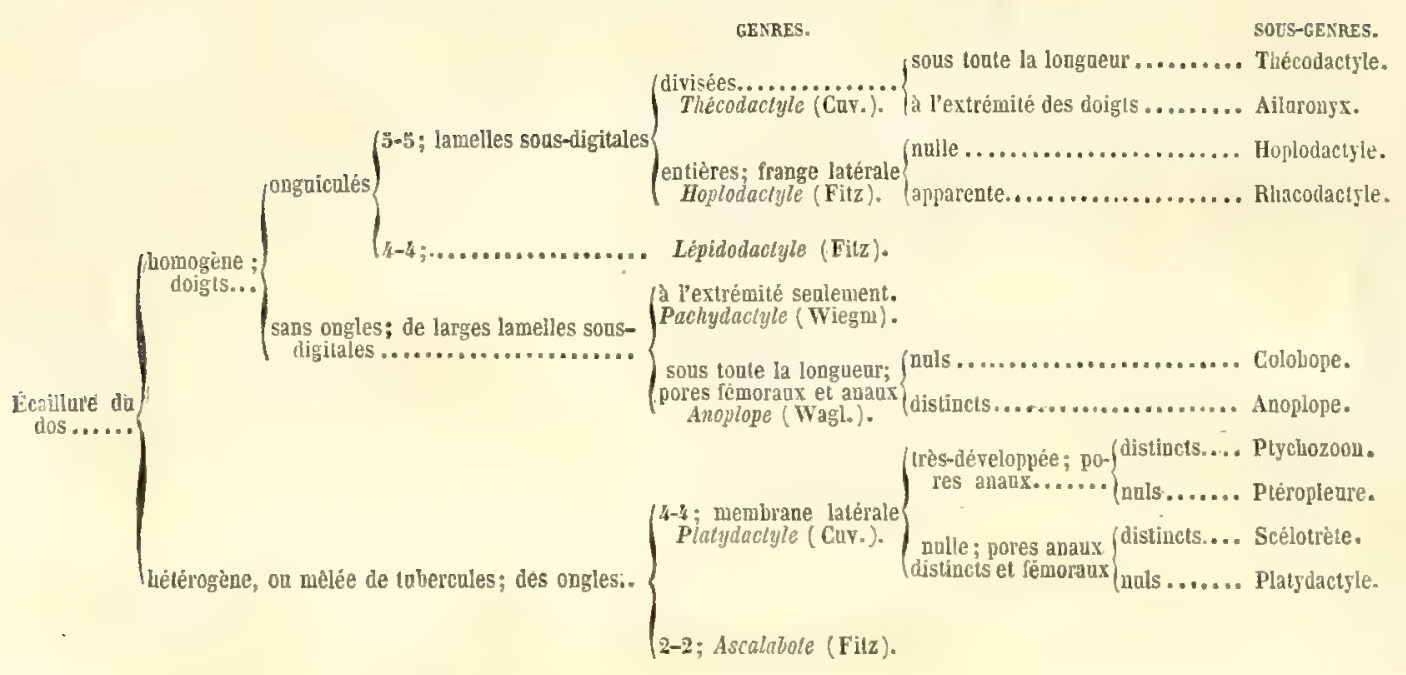

Famlle IV. HéMidactyliens.

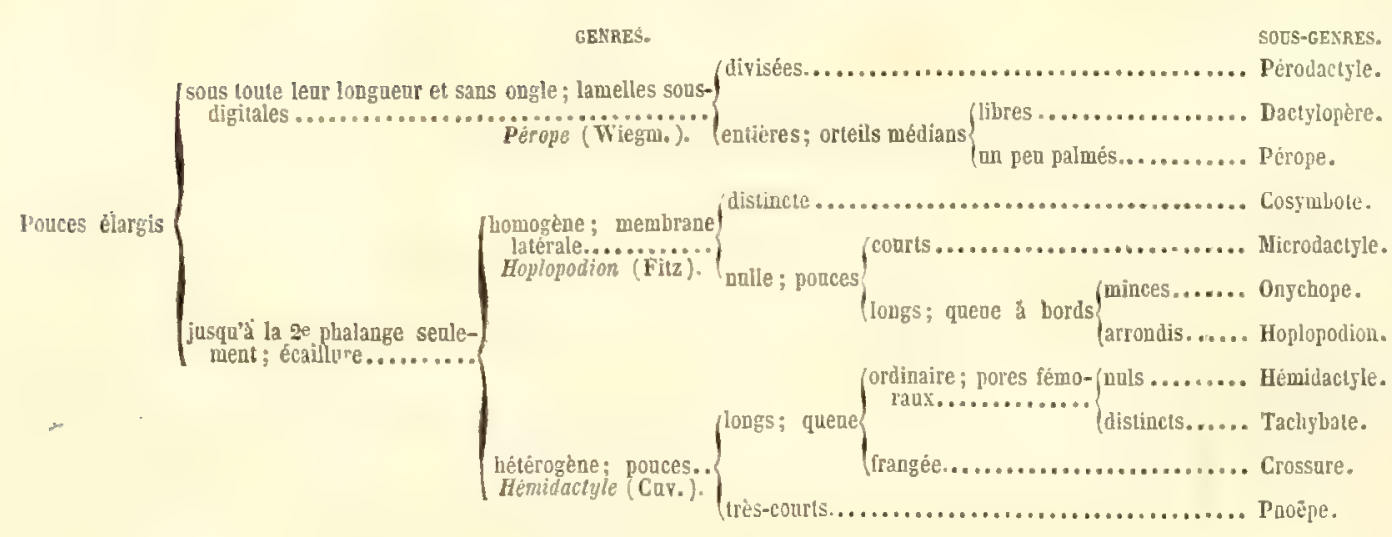


Afin de bien préciser la relation des vingt-deux genres et des quarantetrois sous-genres avec les nombreuses espèces de cette famille, je transcris plus loin les indications fournies par M. Fitzinger luí-même qui, sans décrire ces espèces, les a toutes énumérées.

Je trouve ainsi l'avantage, en présentant la classification de ce zoologiste dans son ensemble et d'une manière complète, de montrer que les caractères employés par lui pour distinguer les sous-genres entre eux sont presque toujours ceux qui ont permis à mon père et à Bibron de séparer nettement les espèces, sans qu'ils se soient écartés de la classification si simple, dont Cuvier a, le premier, posé les bases. Il est facile de le vérifier en comparant la liste suivante des sous-genres avec celle des espèces établies d'après les caractères énoncés dans chacun des six tableaux synoptiques insérés aux pages $294,348,377,39 r$, 402 et 4 ro du t. III de l'Erpét. génér.

A l'exemple de M. Fitzinger, je laisse à toutes les espèces d'un même genre le nom qui se rapporte à cette division générique malgré les différences de dénomination que semblerait devoir nécessiter le partage de chacune de ces grandes coupes en un nombre variable de sous-genres.

J'emploie des caractères italiques pour les espèces dont l'indication est postérienre à l'année 1836 , où le t. III de l'ouvrage de mon père et de Bibron a paru et pour celles qu'ils n'ont pas cru devoir admettre.

1. STEIODACTYLI (6 gen, 21 species).

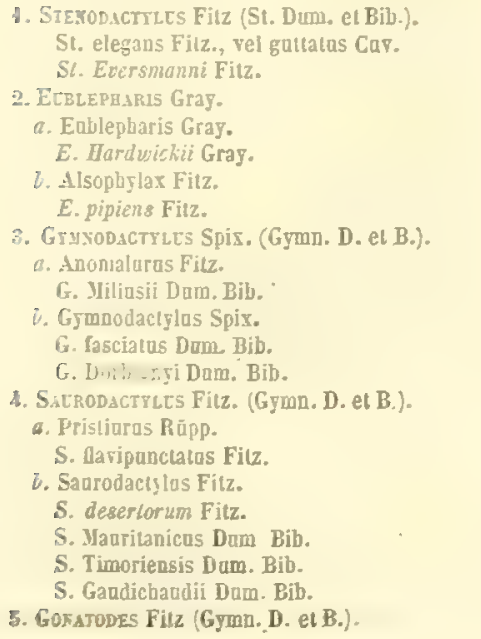

G. Gravenhorstiz Fitz.

G. lavis Filz.

G. albigularis Fitz.

6. Gonyophctylus Filz. (Gymn. D. el B.).

a. Phylluras Car.

G. platuras Fitz.

๖. Dasyderma Filz.

G. spinnlosits Fitz, vel scaber (D. et B.).

c. Gonyodactylus Kuhl.

G. marmoratas Kahl

d. Cyrtorpodion Fitz.

G. Cyprius Fitz.

G. Seaber Fitz.

e. Cyrtodactylus Gray.

G. pulchellus Wagl.

11. PTYODACTYLI (6 gen., 19 species).

1. Spgerodactylos Cav. (Sph. D. et. B.)

Sph. fantasticus Cuv.

Sph. cinereus vel panctatissimas Cuv. Spli. spatator Cav.

2. Diplodacryuus Gray (Phyll. D. et B.).

a. Pachyuras Filz

D. Lesuearii Fitz. 
b. Djplodactylus Gray.

D. vittatus Gray.

D. gerrhopygus Wiegm.

3. Phrllodactrlus Gray, (Phyll. D. et B.

a. Euleptes Fitz.

Ph. Wagleri, vel europaus Fitz.

b. Phyllodactylus Gray.

Ph. porphyreus Wiegm.

Ph. Peronii Fitz.

Pb. Gymnopygus D. et B.

4. Discodact rlus Fitz (Plyyll. D. et B.)

a. Discodactylus Fitz.

D. pulcher Fitz.

D. Cuberculosus Fitz.

乙. Strophurus Fitz.

D. Dumerilii Fitz, vel Gymn, stroph. D. et B

5. Ptrodactylus Cuv, (Pt. D. et B.).

a. Ptyodactylus Wagl.

Pt. guttalus, vel Halsequistii Rüpp.

Pt. lobatus Cav, vel Halsequistii Rüpp.

b. Chiroperas Wiegm.

Pl. Sarrube Fitz.

c. Rhacoëssa Wagl.

Pt. fimbriatus D.et $B$.

6. Olacerds Fitz.

a. Lonchurus Fitz.

0 . limeatus Fiz.

b. Oiacurus Fit\%.

0. Feuilløi Fitz.

III. PLATYDACTYLI ( 7 gen。, 23 species).

1. Thecodactrues Cav. (P). D. et B.)

a Thecodactylus Cuy.

Th. levis, vel theconyx Cav.

b. Ailaronyx.

Th. Seycliellensis Fitz.

2. Lepipodactules Fitz (PL. D. et B.)

L. lagubris Filz.

3. Pachrdactrlus Wiegm. (PI. D. el B.).

P. Bergii, Wiegm., vel ocellatus 0ppel

4. Anopuopus Wagl. (Pl. D. et B.).

a. Colobopus Filz.

A. ornatus Filz.

A. imunguis Waglø, vel Pl. ocellatus 0 ppel.

b. Anoplopus.

A. Cepedeanus Wagl.

5. Hoplodactylus Fitz (PL. D. el B.).

a. Hoplodactylus Fitz.

H. Duraucelii Fitz

b. Rhacodactylus Fitz.

H. Leachianus Fitz.

6. Puatrdactylus Cor. (PI. D. et B.

a Ptychozoon Kubl.

Pl. homalocephalus Cav.

Pl. Hasscltii Fitz. b. Pteropleura Gray.

Pl. Horsfeldii Fitz, vel homalocephalus.

c. Scelotretus Fitz.

Pl. Madagascariensis Gray.

Pl. viltatus Cuv.

Pl. bivittatus D. et B.

Pl. monarchus Schl.

d. Platydactylas Fiiz. (Cuv.).

Pl. Japonicus Schl

Pl. Reevesii Gray.

Pl. guttalus Cav.

7. Ascalabotes Fitz (Plat. D, et B.).

A. Egyptiacus Fitz

A. Delalandii Fitz.

A. fascicularis Schn., vel muralis Gur.

A. Milberti Fitz.

IV. HEMIDACTYLI ( 3 gen., 23 species).

1. Peropos Wiegm. (Hem. D. el B.).

a. Perodactylus Fitz.

P. Oualeusis Fitz.

b. Dactyloperus Fitz.

P. variegatus Fitz.

P. Peronii Filz.

c. Peropus.

P. mutilatus Wiegm.

2. Hoplopodron Fitz. (Hem. D. et B.).

a. Cosymbotus Filz.

H. platyurum, vel marginatum Fitz.

b. Microdactylus Fitz.

H. Peruvaniun Filz.

c. Onychopus Fitz.

H. Garnotii Fitz.

d. Hoplopodion.

H. Cocteaui Fitz.

H. Ruppellii, vel flaviride Fitz.

3. Hemidactreus Cav. (Hem. D. et B.).

a. Hemidactylus.

H. Dorbignyi Fitz.

H. verruculatus Cuy.

H. granosus Rüpp.

b. Tachybates Filz.

H. triedrus Cav.

H. maluya Cuv.

H. Nallereri Filz.

H. armalus Wagl.

H. argyropis Tilesius.

H. Leschenaultii D. et B.

H. maculatus D, et B.

H. luberculosus Wagl.

c. Pnoëpus Filz.

H. Javunicus Cav, vel frenatus.

H. Bojeri Fitz, vel frenalos.

d. Crossurus Wagl.

H. caadiverbera, vel Hem. Sebæ D. et B.

Cette liste comprend 86 espèces. Il est utile de faire observer que sur vingt-cinq marquées par des caractères italiques, comme n'étant pas inscrites dans l'Erpét. génér., qui en contient soizole seulement, il n'y en a que nevf réellement nouvelles. Dans ce nombre, on en compte cinq que M. Fitzinger a 
le premier signalées : ce sont les Saurodact. desertorum, Gonatodes lavis, Gonyodact. cyprius, Hemidact. Nattereri, Platyd. Hasseltii. Pour cette dernière, il n'a que des indications peu certaines, et elle manque au Musée de Vienne.

Quant au Phyllod. europceus, Wagl., non signalé dans l'Erpét. génér., il le considère comme identique à l'espèce qu'il avait antérieurement nommée Prodlact. caudivolvulus, puis Euleptes Wagleri, et qui devient dans son Système le P'hyllodact. Wagleri.

Enfin, M. Fitzinger admet, avec M. Gray et avec M. Gravenhorst, les trois espèces qu'ils ont fait connaître sous les noms suivants $\div 1^{\circ}$ Eublepharis Hardwickii, Gray; $2^{\circ}$ Slenodactylus brachypus, Graven. (placé par le zoologiste autrichien dans son genre Gonatode, Gonat. Gravenhorstii, Fitz.); et $3^{\circ}$ Platyd. (Gecko vel Phelsuma) madagascariensis, Gray.

Les seize autres espèces, dont les noms ne figurent pas dans l'Erpét. génér., en ont été éloignées par différents motifs. Ainsi, pour quelques-unes, c'est en raison de la difficulté que les auteurs de cet ouvrage ont éprouvée relativement à leur classement convenable. Tels sont : I. Stenoductylus Eversmanni, et 2. Eublepharis pipiens, ainsi désignés, en dernier lieu, par II. Fizinger qui, les ayant d'abord confondus, les sépare maintenant d'après l'examen d'échantillons renfermés dans les musées de Berlin, de Vienne et de Saint-Pétersbourg; mais on ne les connaît pas à celui de Paris, et la mème incertitude y reste, comme en 1836, sur leurs véritables caractères. - Il en est de même pour le Gymnodactyle geckoïde de Spix qui, considéré par mon père et Bibron, puis par M. Gray, comme probablement identique au Gymnodactyle rude, forme, pour M. Fitzinger, une espèce distincte d'après l'examen de sujets Brésilıens conservés dans les collections de Munich et de Vienne, et qu'il y rapporte en leur donnant le nom de Gonyodact. spinulosus (3). - Il y a plus de vague encore touchant le Sarroubé de Lacépède (Ptyodactylus Sarrube, Fitz. (4), dont on ne peut rien dire de précis et qui pourrait bien n'ètre, selon la supposition des auteurs de l'Erpét. génér., qu'un Pryod. frangé.

Je n'insiste pas sur les espèces comprises dans la classification de M. Fitzinger sous les dénominations suivantes : 5 Anoplopus inunguis, Wagl. ou Gecko inunguis, Cuv., et qui est un synonyme du Pachydact. Bergii, Wiegm. ou. Platyd. ocellatus, Cuv. - 6 Pteropleura Horsfieldii, non distinct du Pla- 
tyd. homalocephalus, Cuv. - 7 Platyd. Reevesii, Fitz. ou Gecko chinensis, Gray, dont j'aurai plus tard occasion de parler à propos du Platyd. à gouttelettes, Cuv. - 8 Hemidact. granosus, Rüpp., synonyme de l'Hémidact. verruqueux, Cuv. - 9 Hemidact. armatus, Wagl., synonyme du Gecko mabouia, Moreau de Jonnès.

Je dois enfin appeler l'attention sur l'importance extrême que M. Fitzinger attache à la distribution géographique. Souvent, en effet, il rapporte à des espèces distinctes, mais sans énoncer les caractères spécifiques sur lesquels il s'appuie, des individus dont la séparation ne semble motivée que par la différence d'origine. Ainsi les Phyllodact. porphyrés recueillis en Australie et déposés par Péron au Musée de Paris, deviennent les types d'une espèce distincte : Phyllodact. Peronii, Fitz. (10); - les Platydactyles Cépédiens reçus à Londres de l'Océanie, mais cependant non mentionnés en i 845 dans le Catal. de M. Gray, conservent la qualification spécifique particulière que ce dernier leur avait d'abord donnée, car M. Fitzinger les inscrit sous les noms suivants: Anoplopus ornatus, Fitz. (Gecko vel Phelsuma ornatus, Gray (I ). De mème pour l'Hémidact. bridé Schl., il rend le nom proposé d'abord par Cuv. (Hemidact.javanicus) aux individus indiens, et ceux de l'Afrique australe ou de Madagascar et de Maurice forment une espèce particulière : Hem. Bojeri, Fitz. (12). Il nomme Hémidact. de D’Orbigny (13) un Gecko du Chili que les auteurs de l'Erpét. génér. n’ont pu séparer du verruculeux; il ne laisse ce dernier nom qu'aux individus européens, et à ceux qui ont été recueillis dans la Natolie en Asie ou dans l'Algérie. 11 en éloigne les exemplaires d'Égypte, d'Abyssinie et de Sénégambie, et se sert pour les désigner du nom de Hem. granosus (1 4) proposé par Rüppel. Il admet comme Hem. argyropis (1 5) le type australien de l'espèce qui porte au Musée de Saint-Pétersbourg la dénomination proposée par Tilesius: Gecko argyropis et qui se trouve ainsi séparé des sujets indiens dits Hemidact, de Leschenault, Dum. Bib. Enfin, l'Hémidact. tacheté, Dum. et Bib., réunissant des Geckos de l'Inde et du sud de l'Afrique ou de Maurice, les sujets africains conservent ce nom spécifique changé en Hemidact. tuberculosus, Wagl. (16) pour ceux du continent indien.

M. Gray qui, en 1827 , avait apporté déjà des changements au classement de cette famille si naturelle, lui a fait subir, depuis cette époque, des modifications successives qu'il a exposées en détail ei d'une façon méthodique dans 
le Catalogue of the specimens of Lizards in the collect. of the Br. Museum, juin 1845 .

Voici les grandes divisions qu'il adopte et le rang qu'il assigne à ces Sauriens.

Sectio I. Squamata. - Ord. I. Saura. - Subord. II. Pachyglossce. Trib. III. Nyctisaura. - Fam. XXII. Geckotide, 40 gen. - Sect. A. Thecadactylina (8 gen.); B. Hemidactylina (9 gen.); C. Platydactylina ( I gen.); D. Gonyodactylina ( 10 gen.); E. Stenodactylina (1 gen.).

Dans cet arrangement où les coupes génériques sont beaucoup plus nombreuses que dans celui de M. Fitzinger, qui n'en a proposé que vingt-deux, les différences portent quelquefois sur des caractères d'une importance secondaire, et ce sont, le plus souvent, les mềmes que ceux dont Cuvier, ou les auteurs de l'Erpét. génér. ont fait usage pour la distinction des espèces. On peut voir par les noms des zoologistes placés à la suite de chacun des genres, la large part que M. Gray a prise, et par ses travaux anciens, et par ceux d'une date plus récente, à ce morcellement des grandes coupes de Cuvier.

De plus, les genres admis par les devanciers du zoologiste anglais sont souvent modifiés par lui, car c'est quelquefois pour différentes espèces comprises-dans-un de ces genres qu'il a établi de nouvelles coupes génériques.

Afin de rendre plus facile la lecture de cette classification proposée par M. Gray, et pour que l'on puisse saisir dans leur ensemble les caractères d'après lesquels les genres sont établis, je l'ai disposée sous forme de tableau synoptique, comme on le voit à la page suivante.

Je donne ensuite, comme je l'ai fait pour la classification de M. Fitzinger, une lisie complète des espèces admises par M. Gray en inscrivant avec des caractères italiques celles qui n'avaient pas encore été signalées jusqu'alors, et dont le nombre est de quarante-trois sur cent une que cette liste renferme. 


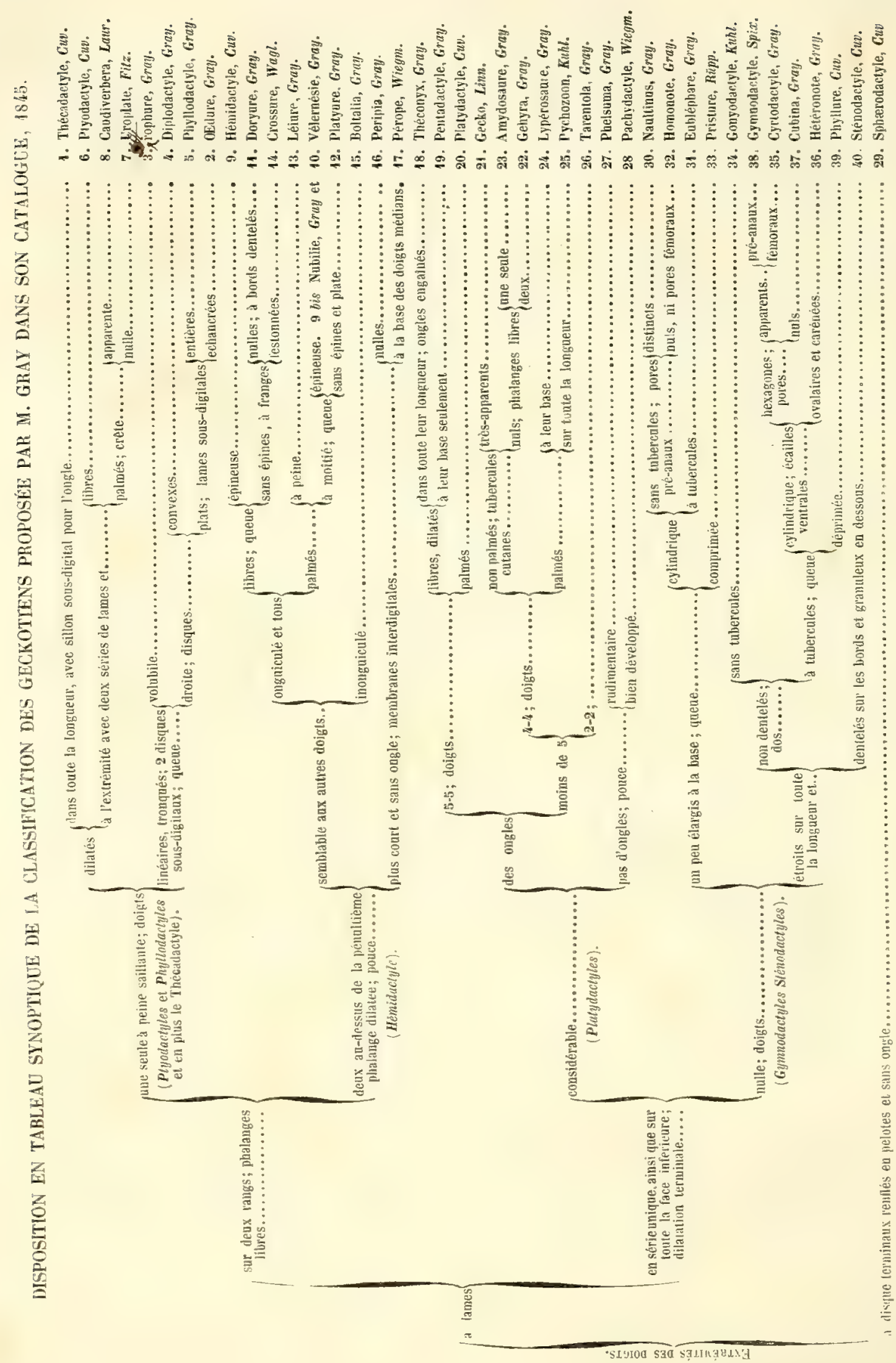


I. THECADACTYLINA (8 gen., 16 species).

1. Thecadetruos Cuv. (Platyd. part. Dum. el Bib.). Th. rapicaudus Gr. (PI, thecongx D, B.).

2. OEnira Gr.

Q. marmorata $\mathrm{Gr}$

OE. rhomlifer Gr." (Phyll. Lesneurij. D. B. ?)

3. STROPHURA Gr. (Phyll. part. D. B.).

S. Sp nigerd Gr. (Ph, stroph. D, B.).

4. Diplodactrues Gr. (Pbyll. part. D. B.).

D. ritlatus $\mathrm{Gr}$.

D. ornalus $\mathrm{Gr}^{\circ}$

D. ocellatus Gr.

D. marmoratus Gr. (porphyreus part. D, B. ?).

D. bil nealus $\mathrm{Gr}$.

D. lineatus $\mathrm{Gr}$.

D. gerrbonygus Wiegm.

5. Pexllodactrlos Gr.

$\mathrm{Ph}$ pulcher Gr.

$\mathrm{Ph}$. Uuberculatus Wiegn.

6. Ptrodactruts Cuy.

Pt. gecko, Gr., vel Hasselquistii D. B.

7. Croplates Filz. (Ptsod. part. D. B.).

L. finbriatus Fitz.

U. li vealus Gic.

8. Caudrerbera Gr. (Caudiverb. part. Laur.)

C. peruviana Lanr. ( Pt. Fenilloel. D. B.).

I1. HEMIDACTYLIVA ( 9 gen., 22 species).

9. Heyioncters Cur.

H. trieurus Les"in. - H. frenatus Schlegel。

H. maculatus D. B. - H. Leschenaultii D. B.

H. Brookii Gr. - H. villahs Gr.

H. depressus Gr. - H. Bellii Gr.

H. verruculatus Car.-H. peruvianus Wiegm.

H rasciulus Gr.

B. mabouia Cuv.

H. mercalorius Gr.

9 bis. Nebilia Gr.

N. Arlentii Gr.

10. YelersesL Gr.

V. Richardsonii Gr.

11. Doryera Gr. (Hemid. part. Cav.) D. Bowringii Gr.

D. Garuotii Gr. (H. Garn. D. B.)

12. Platrore Gr. (Hemid. part. Cuy.)

P. Schneideriauns Gr. (H. Margin. Wiegm.).

13. Leicrus Gr.

L. onnalus $\mathrm{Gr}$

14. Crossurus Wagl. (Candiverb. part. Laur.). C. caudiverliera. Wagl. (H. Sebæ. D. B.).

15. Dostala Gr. Hem. pirt. Cav.). B. sublartis Gr. (H. Coctæi. D. B.?).

16. Peripls Gr. (Hemid. part. Cav.). P. Peronii Gray. (H. Per. D. B.) P. varipgati Gr. (H var D. B.)

17. Pefor os Wierm. (Hemid. part. D. B.) P. matilalus Wiegm.

1II. PLATVDACTYLIMA (12 gen, 32 species).

18. Taecosrs Gr. (Platyd. part. D. B.). Th Seychellensis Gr.

19. Pextadactruos Gr. (Platyd. part. D. B.). P. Davaucelii Gr.

20. Platroactrles (Plat, part. D. B.) P. Leachlanus ciuy.

21. Gecko Linn. (Plat. part. Cav.).

G. verı as Merr. (Pl. galtalas Cav.).

G Reevesii Gr.

G. cbinensis Gr. (Pl, japonicas Srhlo).

G. monarchus. Gr. (P!, mon. Schl.).
G. Smilhis Gr.

G. viltalus Gr (Pl. vĩt. Cuv.).

G. hivitatus Gr. (Pl. bivitt. D. B.).

22. Amroosaurts Gr. (Plat. part. D. B.)

A. lugubris $\mathrm{Gr}$.

23. GenYra Gr. (Peronus part. Wiegm.)

G. oceanira Gr. (Hem. outlensis D. B.?). G. anstralis Gr.

24. Luperosaurus Gr.

L. Cumingii Gr.

25. Ptrchozoon Kubl. (Plat. part. Cuy.)

P. hom lucephalus Kuht

26. Tarentola Gr. (Plat. part. Cuv.)

T. vantianica Gr. (P)at. nuralis. D. B.).

T. xgspliara Gr. (Plat. æg. Cuv.)

T. Delatandii Gr. (Pl Del. D. B.).

T. amerivana Gr. (Pl. Niłbertii D. B.).

T. Burneensis Gr.

T. clypeala $\mathrm{Gr}$.

27. Phelsum. Gr. (Platyd, part. æg. Cuv.),

Ph. Cepredianum Gs.

Ph. Merdagascariense $\mathrm{Gl}^{\mathrm{B}}$

Ph. Uneatuu Gr. (Pl. oceilatus. var. D. B.).

28. Pacaydictylus pait. Wiegm (Plat. part. Cuy.)

P. ocellatus Gr. (1'l. ocellatus $0 \mathrm{pp}$.)

P. marcululus Gr.

P. elegans $\mathrm{Gr}$.

29. SpHerodactyuns Cav.

S. sucuialor Cav.

S. fantastirus Cuy

S. punchulissinus D. B.

S. nigropunctatus $\mathbf{G r}$.

S. Richardsonii Gr.

IV. GONYODACTYLINA ( 10 gen., 22 species.).

30. Naultinus Gr

N. pacificus Gr. (PI. Duvaucelii D. B. ?).

N. eleyans $\mathrm{Gr} .-N$. granuluhs $\mathrm{Gr}$.

N. Grayii Bell. - N. brevidaclylus Gr

$N$, punclutus $\mathrm{Gr}^{\mathrm{r}}-\boldsymbol{N}$. maculatus $\mathrm{Gr}$.

31. Eurlepharis G:

E. Haidwickii Gr. - E. Derbianus Gr.

32. Howonota Gr. (Gymmou. part. D. B.).

H. Gaudichaulii Gr.

33. Pristuros Rüpp. (Gymnod. part. D. B.)

P. Mlavipunctitus Rūpon.

34. Gontodictruts pat! Kuhl.

G. Timorensis Gr. (Gymn. Tim. D. B.).

G. auslralis Gr

G. alboyularis Gr. (Gymn. all. D. B.).

G. ? ocellatus Gr. (Cyrtod, ocell. Gr.).

G. ? manrilas icas Gr. (Gymn maar. D. B.).

35. Crrtodactruus Gr. Gonyod. part. Kuhl, el Gymn.

part. D. 8.

C. marnolatus Gr.

C. palchellus Gr.

36. Heteronita Gr.

H. Kentallii $\mathrm{Gr}$.

H. Binoti Gi

37. Cobina Gr. (Gymu. part D. B.).

C. fascista Gro-Cr. D Orbignii, Gr. - C. Darwint, Gr

38. Granod ictrlos Spix. (Gymn. part. D. B.)

G. reckoides Spix.

39. Phruluals Cuy (Gymmod. part D. B.)

Ph. platerus Cuv. (Gymmod, phyil. D. B.).

Ph. Milıasii Bory.

$\mathrm{Ph}$. inermis $\mathrm{Gr}$.

v. STENonaCTylina. (1 gen., 1 species).

40. Stenodactylus Guv.

St. gutlatus Cav. 
En I 850 , S. A. le prince Ch. Bonaparte, dans le tableau qu'il a publié à Leyde, sous ce titre: Conspectus systematum herpetologia et amphibiologioe, place les Geckos en tête de la troisième section des Reptiles ou Squamata. Ordo 6. Saurii. - Tribus I. Pachyglossi. - I2. Gecconidæ. Ce groupe est partagé en 4 familles qui, dans l'ensemble du système, prennent les numéros:

20. Hemidactylina (20 espèces cosmopolites). - 21. Platydactylina ( 20 esp. cosmopol.). - 22. Ptyodactylina (20 esp. cosmopol.). - 23. Gymnodactylina ( 8 esp. As., Afr., Oc., Amér.).

Je ne mentionne ici que les travaux d'ensemble où la classification des Geckotiens a été exposée dans tous ses détails. C'est à propos de certains genres et de certaines espèces que j'aurai à citer plus tard les publications de MM. Th. Bell, Berthold, Bianconi, Bibron et Cocteau, Cantor, Eichwald, Gené, Gosse, Guichenot, Kelaart, Peters, A. Smith, Troschel qui, dans des faunes particulières ou dans des études spéciales, ont fait connaître des Geckotiens jusqu'alors inconnus, ou bien ont donné de nouveaux détails sur des espèces déjà décrites.

Je vais maintenant passer successivement en revue:tous les genres de cette famille.

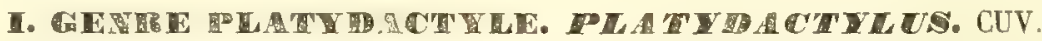

Ce premier groupe, comprenant les Geckos à doigts élargis dans toute leur longueur, correspond à la troisième famille de la classification de M. Fitzinger: Platydactyli (voy. le tableau, p. 442), et à celle de M. Gray : Platydactylina. Ce dernier en éloigne cependant le Plat. theconyx de Cuvier pour le placer en tête d'une autre famille (Thecaductylina), et il fait.entrer dans celle des Platydactyles le genre Sphériodactyle.

Le genre Platydactyle s'est enrichi d'un assez grand nombre d'espèces qui, pour la plupart, sont inconnues au Musée de Paris.

On peut, en les groupant d'après l'absence ou la présence des ongles et, dans ce dernier cas, d'après leur nombre, ew présenter l'énumération suivante :

I. Platydactyles inonguiculés.

$1^{\circ}$ A pouces bien déreloppés, dont le type est le Plat. ocellé (Plat. ocellatus, vel inunguis, Oppel. et Cuv. Pachydactylus, Wiegm.). - 9 espèces.

Pach)dact)lus Bibronii, Smith (lllustr. of the Zool. of S. Afr:, pl. L, 
fig. I). - Pach. capensis, Smith (Ill., fig. 2, mentionnés l'un et l'autre, par erreur, dans l'ouvrage du zoologiste, comme l'indique la table, sous le nom de Tarentola). - Pach. rugosus, Smith (Id., pl. Lxxv, fig. 2). - Pach. mariquensis, Smith. - Pach. formosus, Smith. - Pach. mento-marginatus, Smith. (1d. Appendix, p. 4 et 5). - Pach. maculatus, Gr. (Cat. of Liz, p. 167). - Pach. elegans Gr. (Id., p. 168). - Pach. punctatus, Peters (Monatsber. der kôn. Preuss. Akad. der Wissensch.zu, Berlin, 1854, p. 6 15.) $2^{\circ} \mathrm{A}$ pouces rudimentaires, dont le type est le Plat. cépédien, Cuv. (Gecko cepedianus, Péron). - Anoplopus, Wagl.). - 2 espèces.

Phelsuma madagascuriense, Gr. (Cat., p. I66.) Esp. douteuse. - Phels. lineatum, Gr. (Id., p. I66.) Espèce douteuse.

II. Platydactyles à deux ongles seulement, dont le type est la Geckotte de Lacép. ou Plat. des murailles, Cuv. - (Ascalabotes, Fitz.) - 2 espèces.

T'arentola Borneensis, Gr. (Cat., p. 165). - Tar. clypeata, Gr. (Id., p. 166$)$.

III. Platydactyles à quatre ongles, dont le type est le Plat. à gouttelettes, Cuv. (Plat., Cuv., part.). - 7 espèces.

Plat. stentor, Cantor (Cat. of Malayan rept., p. I8). - Gecko Walbergii, Sm. (Loc. cit., pl. Lxxv, fig. I). - Gecko Sinithii, Gr. (Cat., p. 162). Gecko Reevesii, Gr. (Cat., p. r6r). Esp. douteuse. - Gehyra australis, Gr. (Cat., p. ı63). - Lyperosaurus Cumingii, Gr. (Cat., p. ı63). Espèce à doigts palmés à leur base. - Ptychozoon Hasseltii, Fitz. (Syst. Rept., p. 101). Espèce à membrane latérale voisine du Plat. homalocéphale et donteuse.

IV. Platydactyles à cinq ongles et à lamelles sous-digitales entières. 3 espèces.

Naulinus pacificus, Gr. (Cat., p. 169). - Plat. trachygaster, A. Dum. Cat. méth. des Rept., p. $35, n^{\circ} 5$ bis). - Plat. Boivini, A. Dum. Esp. nouv.

Les trois nouvelles espèces composant ce dernier groupe sont les seules que le Musée de Paris possède, et ce sont, par conséquent, les seules aussi dont j'aie à parler, parmi les vingt-trois que je viens d'énumérer, en comprenant dans ce nombre les quatre espèces qui sont douteuses, et dont une seule (Plat. Reevesii) devra nous arrêter pendant quelques instants. 


\section{[. -5 bis 1 . Platydactyle a ventre rude, P. trachygaster, A. Dum.}

Idem., A. Dum. (Cat. méth. de la collect. des Rept. du Mus. de Paris, p. 35).

Pl, xvir, fig. 1, portion de l'abdomen.

Peau du dos à grains plus ou moins saillants ; région abdominale couverte de grains convexes, un peu proéminents, d'où il résulte une faible rugosité du ventre; doigts élargis dans toute leur longueur, tous onguiculés, à lamelles entières, dont les terminales sont légèrement infléchies en dehors de la ligne médiane, dans le point où s'ouvre la gaine ablique de l'ongle.

La tête est large; le museau est mousse et arrondi. De chaque côté du corps, depuis l'angle de la mâchoire jusqu'à l'aine, la peau forme un pli peu considérable, mais très-nettement dessiné. Le dos ne porte pas de sillon médian.

La teinte générale est un brun fauve, plus clair en dessous qu'en dessus, où elle est plus foncée le long de la région vertébrale que partout ailleurs.

Ces différences de caractères indiquent les analogies et les dissemblances de cette espèce et des autres Platydactyles.

Le caractère le plus remarquable est fourni par la disposition tout exceptionnelle de l'écaillure du ventre, laquelle, au lieu d'être formée, comme chez tous les Platydactyles, de petites squames plates, est composée de granulations un peu ovalaires, à grand diamètre transversal, assez volumineuses. Elles sont disposées très-régulièrement, en lignes obliques et séparées entre elles par des granulations moins grandes, et dont on compte six autour de chacune des granulations plus volumineuses, deux supérieures, deux inférieures, et les deux autres aux extrémités de son diamètre latéral. (Voyez pl. xvir, fig. 1.)

Ce Platydactyle est du petit nombre de ceux qui ont cinq ongles, et il est

1. Dans le Cat. méth. de la collect. des Rept. du Mus. de Paris, j’emploie pour la numération des espèces dont la description n'est pas donnée par l'Erpét. génér., les mots bis, ter, et mème quater, en les mettant à la suite du numéro que norte, dans ce livre, l'espèce auprès de laquelle la nouvelle vient se ranger. J'indique, de cette manière, la place naturelle que celle-ci doit occuper dans la classification, en évitant de troubler la série ordinale primitive. Je me sers ici de la numération adoptée dans le Catalogue quand il s'agit d'espèces qui y sont signalées, ou je classe, d'après la mème méthode, celles qui étaient jusqu'à présent inédites. Le chiffre romain devient un numéro d'ordre spécial joint à chacune des descriptions contenues dans ce deuxième Mémoire. 
facile de le distinguer. $I^{\circ}$ Par l'élargissement des doigts dans toute leur longueur et par l'absence de membranes inter-digitales, il s'éloigne, d'une part, des Plat. de Duraucel et de l'Océan pacifique, et de l'autre, du Plat. de Leach. $2^{\circ}$ Il ne présente pas un sillon sous les doigts, comme le Plat. théconyx. $3^{\circ}$ La gaîne onguéale est fort différente, par sa forme et par sa situation, de celle de l'espèce nouvelle que je décris plus loin sous le nom de Plat. de Boivin. $4^{\circ}$ C'est avec le Plat. des Seychelles, qu'il y aurait le plus d'analogie, en raison de la conformation des doigts qui, dans l'une et dans l'autre espèce, ont un ongle oblique, dont la pointe dirigée un peu latéralement, ne fait pas saillie sur la ligne médiane, à la face inférieure du doigt, mais bien sur le côté, lorsqu'il sort de sa gaine protectrice. Pour les trois doigts internes, la phalange onguéale se porte en dedans, tandis que celle des deux doigts externes se dirige en dehors. Dans le point où se voit l'orifice de cette gaìne, les lamelles sous-digitales sont un peu infléchies. (Voy. pl. xvmi, fig. 5 montrant la face inférieure de l'un des doigts du Plat. des Seychelles).

C'est en raison de cette disposition, que M. Fitzinger a placé dans le genre Thécadactyle, Guv., le Plat. des Seychelles, qui y devient le type du sousgenre Ailuronyx (A.Seychellens.), dont tous les caractères ne se retrouvent pas dans la nouvelle espèce. Tels sont cenx-ci : Pores anaux distincts; point de pli sous la gorge, ni sur les parties latérales du tronc (Syst. Rept., p. 98).

C'est le même caractère tiré de la gaîne onguéale qui a motivé l'établissement du genre Théconyx, Gr. pour le Plat.des Seychelles, (Cat. of Liz., p. 159 ).

Le caractère tiré de la direction de l'ongle, est le seul qui rapproche réellement les deux espèces dont il s'agit. Le Plat. à ventre rude s'éloigne, en effet, du précédent par la forme plus élargie de la tête terminée par un museau beaucoup plus arrondi et plus mousse. Les grains de la peau ne sont pas coniques, ni aussi serrés. Au lieu d'un seul rang de plaques derrière celles qui garnissent la lèvre inférieure, il y en a trois. Le dos ne présente pas de sillon. Le ventre enfin porte un système de granulations tout à fait spécial.

Le Musée de Paris ne possède qu'un seul spécimen de cette nouvelle espèce qu'il a reçue de Madagascar. Elle est consignée, Répert. Erp.gén., t. IX, p. 248.

Sa longueur totale ne peut pas être exactement appréciée, car la queue a été brisée, et celle qui l'a remplacée paraît plus courte qu'elle ne. semblerait devoir l'être. Le tronc et la tête ont ensemble une longueur de $\mathrm{o}^{\mathrm{m}} \mathbf{1} 6$. Archives du Musétu. T. Vili. 


\section{II. - 5 ter. Platydactrle de Bonvin, Plat. Boivini, A. Dum. ESPÈCE NOUVELLE.}

Pl. xvir, fig. 2, 2a, 2b, pcur la conformation des doigts.

Granulations du dos et desfancs entremélées de tubercules peu saillants et assez irrégulièrement disposés; doigts dilatés dans toute leur longueur, garnis en dessous de lamelles transversales entières, si ce n'est aux pouces; poriant tous un ongle rétractile, protégé par une sorie de gaine formée par 2 grandes écailles latérales convexes, et par une $3^{e}$ écaille placée en dessits.

La tête est plate, un peu allongée; le museau est conique. Il n'y a pas de plis cutanés sous la gorge, ni sur les flancs; on en voit deux sur le cou senlement; l'un commence au-dessus du conduit auriculaire dont il continue le bord snpérieur, l'autre, un peu plus inférieur, rejoint le précédent au niveau de l'épaule où ils se perdent.

Les plaques de la lèvre supérieure sont au nombre de douze, de chaque côié de la rostrale, qui est beaucoup plus longue que hante, et dont le bord supérieur présentè dans sa partie moyenne une saillie sur le milieu de laquelle s'appuie une petite plaque ovalaire. De chaque côté, ce bord supérieur se relève obliquement et se trouve en contact avec une plaque plus grande que celle du milieu. Cette dernière est enchâssée dans l'espace libre résultant de l'intervalle qui séfare les deux plaques latérales.

A la lèvre inférieure, il y a dix plaques à droite comme à gauche de la mentonnière. On voit derrière elles un rang unique de grandes écailles sem. blables, mais moins nombreuses et suivies de grauulations fines qui revêtent les régions scus-maxillaire et gulaire.

Les membres sont forts et trapus, et la particularité la plus intéressante qu'ils offrent à noter, consiste dans la conformation de cette sorte de gaine où l'ongle se cache pendant sa rétraction. (Voy. pl. xvir, fig. $2,2 a$ et $2 b$, montrant fort amplifiée cette curieuse structure.)

Les ponces ont une apparence un peu différente, car les lamelles inférieures, au lieu d'être entières comme aux autres doigls, sont en chevron et même les plus antérienres sont divisées, et c'est dans ce sillon qu'on tronve l'ongle, qui n'est pas reçu, comme aux autres doigts, dans une gaîne terminale.

Il n'y a point de pores le long de la face interne des cuisses, ni au-devant du cloaque sur l'échantillon unique du Musée de Paris. A la base de la quene, 
il ya, de chaque côté, deux écailles un peu plus saillantes que les autres. La queqe, brisée à pen de distance de son origine, s'est reproduite.

Ia teinte générale, un peu altérée par le séjour dans l'alcool, est un brun faute sur lequel semblent se détacher quelques bandes transversales fines et irrégulières blanchâtres. Les régions inférieures, d’une teinte moins foncée, sont piquetées cà et là de points d'un brun plus sombre.

Tes différents Platydactyles pentonyx connus jusqu'ici, aucun ne peut être confondu avec celui que je décris. Il n'a pas les doigts élargis à la base seulement comme les Plat. de]Dupaucel (voy. pl. xvin, fig. I, I $a$ ), et de l'Océan pacifique; il n'a pas de membranes inter-digitales, comme celui de Leach. Ses ongles ne sont pas obliquement dirigés comme ceux des Plat. des Sejchelles (voy.pl. xvir, fig. 5 ) et trachygastre, ni protégés par une gaîne semblable à celle qu'on voit chez ces denx espèces.

Il offre d'aỉleurs une particularité remarquable. Tandis, en effet, que les différents Platydactyles que je viens de nommer sont homonotes, c'est-à-dire ont une écaillure composée de grains tous égaux entre eux, cette nouvelle espèce, au contraire, est hétéronote, des tubercules étant dispersés au milieu des fines granulations de la peau.

Sa taille est de $o^{\mathrm{m}} 22$ ainsi répartis : tête et tronc, $\mathrm{o}^{\mathrm{m} 2} \mathbf{I} 2$, quene, $\mathbf{o}^{\mathrm{m} 2} \mathbf{1 0}$

Ce Gecko a été recueilli à Madagascar par M. Boivin, qui en a fait don au II uséum avec d'autres Reptiles de la même contrée. C'est en recounaissance des enrichissements apportés à nos collections par ce voyageur, que j'ai émployé son nom comme désignation spécifique.

\section{III. - 6 bis. Platrdactyle de l'Océan PacielQue, Plat. (Naultinus) pacificus, Dum., Bib.}

Tiaultinus pacificus, Gr., Dieffenbach New-Zeal., t. 11, p. 203; Zool. Erebus and Terror; Cat. of Lisards, p. 169. - Plat. pacif., Dum., Cat.des Rept., p. 35, $\mathrm{n}^{\circ} 6$ bis. - Répert. Erp. gin., t. IX, p. 248.

Doigts peu dilatés et seulement jusqu'à la pénultieme phalange; dix plaques sus-labiales de chaque colé de la rostrale, dont le sommet tronqué est surmonté d'une plaque presque circulaire; neuf plaques au bord maxillaine inférieur de chaque cóté de la mentonnière.

La tête est un peu déprimée et le cou légèrement rétréci. Les membres sont robustes et trapus. Chez les mâles, il y a, au-devant du cloaque, trois 
rangées en chevrons d'écailles crypteuses, et à la base de la queue deux ou trois petites épines latérales, beaucoup moins apparentes chez les femelles, où elles sont tout à fait rudimentaires et manquent mème souvent.

La teinte générale est un brun pâle, plus clair en dessous qu'en dessus, marbré et pointillé de brun plus foncé, qui forme, en travers du dos, quatre larges taches irrégulières, quelquefois confluentes, et souvent, sur les côtés du tronc, il règne une bande de même nuance. Du pourtour de l'oeil, il part des lignes foncées : l'une se dirige en arrière et en bas, l'autre, directement en arrière, et va passer au-dessus du tympan.

Le plus grand échantillon du Musée de Paris a $0^{\mathrm{m}} \mathrm{r} / 4$, et la queue entre pour moitié dans cette longueur totale. Cette espèce n'atteint donc pas les mêmes dimensions que le Plat. de Dusaucel, qui peut avoir o $\mathrm{o}^{\mathrm{m}_{2}} 25$ à $\mathrm{o}^{\mathrm{m}} 26$.

Je compare ces deux Platydactyles l'un à l'autre, parce qu'on remarque entre enx quelques analogies. La plus frappante se tire de la structure des doigts, qui ne sont élargis qu'à leur base, et dont l'avant-dernière phalange et celle qui supporte l'ongle sont étroites et rétrécies. Les fig. I et I $a$ de la pl. xvır (main et doigt du Plat. de Duv.), représentent très-exactement cette disposition prise comme point de départ par M. Fitzinger (Syst. Rept., p. Ioo) pour la formation du genre Hoplodactylus, qui ne comprend que cette espèce et le Plat. de Leach, dont les doigts offrent la même apparence (voy · pl. xxvir, fig. 6 de l'atlas de l'Erpét. génér.), mais devenu, à cause de ses membranes inter-digitales, le type de son sous-genre Rhacodaciylus Fitz.

Les différences cependant sont nombreuses entre les Plat. de Dur. et de I Océan pacifique. Ce dernier, en effet, a la tête moins déprimée; il semble rester toujours plus petit, à en juger par les nombreux échantillons de notre Musée. Il n'y a jamais de pores anaux ou fémoraux, et les femelles n'ont point d'écailles crypteuses le long des cuisses. Le système de coloration n'est pas le même; il n'y a pas non plus identité dans le nombre des plaques labiales, puisque dans le Plat. de Dw., en y comprenant la rostrale et la mentonnière, on en compte 25 en haut et 23 en bas, et seulement 2 I et r 9 dans l'autre espèce. Enfin, celle-ci a été recueillie dans la Nouvelle-Zélande, les îles Marquises et la Tasmanie, par MM. de Belligny et Arnoux. C'est également des îles de l'Océanie que le Musée de Londres a reçu les types étudiés par M. Gray, tandis que ceux du Plat. de Duvaucel ont été envoyés du Bengale par le naturaliste-voyageur dont ils portent le nom. 
11. Gray nomme l'espèce dont il s'agit Naultinus pacificus et en rapproche, mais avec doute, le Plut. Luvaucelii, D. et B. On vient de voir les motifs qui s'opposent à cette assimilation.

Quant au genre Naultinus, Gr., il commence la série des Gonyodactyles dans le Cat. du zoologiste anglais. Or, les deux espèces que je compare étant de véritables Platydactyles, elles ne pouvaient conserver cette dénomination générique. Si la structure de leurs doigts devait ètre prise en considération et fournir un caractère qui permit de les placer dans un groupe particulier de la grande division des Platydactyliens, on pourrait, en adoptant le genre Hoplodactyle, Fitz., nommer ce Gecko H. pacificus, et le ranger, pour lui conserver ses véritables analogies, près de l'espèce dite $H$. Duvaucelii, Fitz.

- Parmi les Plat. inonguiculés à pouces rudimentaires, j'ai cité comme espèces non décrites dans l'Erpét. génér., le Phelsuma lineatum, Gr., qui parait n'être qu'une variété du Plat. ocellé et le Phelsuma Madagascariense, Gr. Ce dernier a été mentionné spécialement dans le Cat. des Repl. du Mus. de Paris, p. 3/, comme n'offrant pas des caractères suffisamment distincts pour que les échantillons reçus de Madagascar puissent être séparés de ceux du Plat. Cépédien, qui proviennent des îles Maurice et de la Réunion.

- Dans l'énumération que j'ai faite des espèces à quatre ongles, dont la description ne se trouve pas dans l'Erpét. génér., il y a le Plat. de Reeves (Gecko Reevesii, Gr.).

M. Fitzinger (Syst., p. Iо I), admet cette espèce à l'exemple de M. Gray, mais il en fait remonter la première indication à une époque beaucoup plus ancienne, car il donne comme synonyme Lacerta chinensis, Osbeck. Or, si l'on consulte le texte de ce voyageur, on ne trouve qu'une courte série trèsincomplète de caractères, qui même ne conviennent pas tous au groupe où ce Plat. de Reeves doil être rangé, puisqu'il est très-voisin du Plat. guttalus, si même il ne lui est identique : je veux parler du nombre des ongles. Voici la phrase latine du voyage de Osbeck. (Voy. to China and East-Indies, trad. angl., t. II, p. 67. Londres, 1771). Lacerta (chinensis) cinerea, cauda ancipiti, corpore paulo longiore, pedibus pentadactylis omnibus unguiculatis. Dans la description peu détaillée qui suit cette diagnose, et où l'on trouve l'indication des tubercules cutanés, il est encore dit: les pieds de devant et ceux de derrière ont cinq doigts non palmés, tous armés d'ongles crochus.

Ce Gecko ne peut donc pas être confondu avec celui à quatre ongles que 
M. Gray a considéré comme nouveau, et a décrit sous le nom du voyageur Reeves qui l'a rapporté de Chine, et contrairement à l'opinion de M. Fitzinger, ce Plat. Reevesii ne peut pas avoir pour synonymes Lacerta chinensis, Shaw. - Stellio chin., Schn.-Ascalabotes chin., Id.-Gekko Osbeckii, Merr.

De plus, enfin, il résulte des termes mêmes de la description qu'il est fort difficile, comme mon père et Bibron l'ont fait observer (Erpél. génér., t. III, p. $28 \mathrm{~s}$ ), de reconnaître le Saurien si incomplétement décrit par Osbeck.

Quant au Plat. de Reenes, dont le Musée de Paris possède un exemplaire donné par celui de Londres, et d'autres sujets originaires de Chine étiquetés par Bibron, il est fort douteux qu'il soit le type d'une espèce distincte.

De nombreuses ressemblances le rapprochent du Plat. à gouttelettes. Les différences qui les distinguent sont, au contraire, en petit nombre. Ainsi, chez le Plat. de Reeves, comme Bibron l'avait indiqué dans une note manuscrite, $t^{\circ}$ les granulations sur la peau de la tête et les écailles du dos sont proportionnellement plus petites, tandis que c'est le contraire pour les pièces de l'écaillure ventrale; $2^{\circ}$ la teinte générale des parties supérieures est d'un gris rougeâtre relevé par la grande blancheur des taches régulières, qui for ment sur le dos des lignes transversales.

On ne trouve point d'ailleurs dans le Cat. de M. Gray, p. 16r, l'indication de caractères spécifiques tranchés, et quoiqu'il n'y soit pas question des pores fémoraux pour le Plat. à gouttelettes, ils y existent comme chez les individus qui présentent les particularités que je viens d'indiquer pour les Plat. de Reeves. Ces derniers, dans le Musée de Paris et dans celui de Londres, qui en possède un seul échantillon, sont tous originaires de Chine. On pourrait donc les considérer comme ne constituant qu'une variété de clinut. Celte manière de voir semblerait confirmée par ce fait que nul Plal. à goutzelettes de la collection française ne provient de cette contrée, mais il n'en est pas de même au Musée britannique.

De tout ce qui précède, on peut tirer cetteconclusion, qu'il reste du doute, ainsi que je l'ai dit (Cat. du Mus. de Par., p. 37), sur le rang que ce Gecko doit occuper, soit comme espèce distincte, soit comme variété du Platydac. tyle à gouttelettes. 


\section{GEVIEE HI}

Les Geckotiens qui, en raison de la conformation de leurs doigts élargis seulement à la base et grêles à leur extrémité libre, ont reçu de Cuvier la dénomination que la plupart des auteurs ont adoptée, constituent dans les différents systèmes un groupe bien distinct. M. Fitzinger les considère çomme formant une famille qu'il divise en trois genres comprenant eux-mêmes onze. sous-genres (Voy. le tableau de la Fam., Iv, p. 442). Dans la classification de II. Gray, cette famille renferme dix genres (Voy. le tableau, p. 448).

Ces divisions paraissent trop multipliées, et donnent surtout dans l'arrangement proposé par M. Gray, qui n'admet pas de sous-genres, une trop grande valeur à des caractères qui, le plus souvent, ne sorit fondés que sur des différences spécifiques.

On peut cependant, à l'exemple de Wiegmann, et comme le fait M. Fitzinger, établir une subdivision pour les espèces à pouces courts et en quelque sorte tronqués, en les réunissant sous le nom de Peropus, Wiegm. Tels sont les Hém. oualien, de Péron 1 , varié et mutilé. Si, au contraire, il ne paraît pas très-nécessaire d'accepter la séparation en deux groupes des autres espèces, selon que l'écaillure du dos est homogène on hélérogène, comme l'a proposé le zoologiste de Vienne qui, par ce motif, les a placés soit dans le genre Hoplopodion, Fitz., soit dans le genre Hémidactyle, Cuv. proprement dit, il n'en est pas de même $1^{\circ}$ pour le sous-genre Cosymbotus, Fitz. ou Platyure, Gr., auquel est rapportée l'espèce nommée par Cuvier Hém. borclé, en raison du repli membraneux des flancs, des membres et de la queue, et $2^{\circ}$ pour le singulier Gecko à longue queue festonnée que Laurenti avait pris pour type de son genre Caudiverbera, et qui a été figuré par Séba, dont il a pris le nom, comme désignation spécifique, dans l'Erpét. générale.

On pourrait également, à l'imitation de M. Gray, adopter une dénomination particulière pour l'Hém. oualien, dont les lamelles sous-digitales sont larges, entières, non divisées comme celles de la plupart de ses congénères (Voy. pl. xvir, fig. 8), et le nommer, avec ce zoologiste, Gehyra oualensis,

1. Je dois relever ici une faute d'impression dans le tableau synoptique relatif au genre Hémidactylle (Erpét. génér., t. III, p. 349) où l'espèce à queue déprimée et dédiée à Péron est rangée, à tort parmi celles à queue ronde. 
mais sans le faire sortir, ainsi qu'il le propose, du groupe des Hémidactyles, pour le placer dans celui des Platydactyliens.

Les collections du Musée de Yaris ne possèdent pas tous les Hémidactyles décrits comme nouveaux dans ces dernières années. On peut les rapporter soit à l'un, soit à l'autre des groupes qui sont compris dans ce genre.

A. Espèces à pouce plus court que les autres doigts et comme tronqué. (Dactylopères, Dum. et Bib.). (Peropus, Wiegm.).

Hem. capensis, Smith (Illustr. of the Zool. of S. Afr., pl. nxxv, fig. 3 et 3 a).

B. Espèces à pouce semblable aux autres doigts (Dactylotèles, Dum. et Bìb.). $1^{\circ}$ Ecaillure homogène (Hoplopodion, Fitz.).

Hemidactylus flaviridis, Rüpp. (Neue Virbelth. Abyss.). - Hem. Bellii, Gr. (Cat. of Liz., p. 155). - Velernesia Richardsonii, Gr. (Id., p. 156). Doryura Bowringii, Gr. (Id., p. I56). - Leiurus ornatus, Gr. (Id. p. 157). $2^{\circ}$ Écaillure hétérogène (Hemiductylus, Cuv., part.).

Hem. Nattereri, Fitz. (Syst., p. 105). - Hem Brookii, Gr. (Cat., p. I 53 ). - Hem. depressus, Gr. (Id., p. 153). - Hem.fascialus (Id., p. I54). Hem. mercatorius, Gr. (Id., p. 155). - Hem. vittatus, Gr. (Id., id.). Hem. Bellii, Gr. (Id., id.). - Hem. platycephulus, Peters (p. 6.5), lequel, recueilli dans la province de Mozambique, parait fort analogue à un Gecko envoyé de la même région du continent africain au Musée de Bologne, par M. Fornasini, et que M. Bianconi, malgré la différence de pays, tronve tellement semblable à l'espèce des Antilles, qu'il le nomme Hem. maboria. (Specimina zoologica Mosambicana, p. 19, pl. I (Rept.), fig. I, I $a$, I $b$ ). La fig. I $c$ de cette même planche représente la face inférieure de l'un des doigts de l'Hém. tacheté; mais les plaques sous-digitales n'offrent pas absolument la disposition indiquée dans ce dessin, où elles sont transversales et sans aucume échancrure. Leur véritable apparence est reproduite fig. 7 de la pl. xvır, annexée à ce Mémoire.

M. Cantor (General features of Chusan in Ann. of nat. hist., 1842, t. IX, p. 275 et 482 ), a parlé d'un petit Gecko très-abondant dans ces îles voisines de la Chine et qu'il a nommé Hem. nanus. Il n'a décrit que le système de coloration. M. Gray, d'après l'examen d'un échantillon conservé au Musée de la Comp. des Indes, à Londres, où il a été envoyé par le docteur Cantor, l'a rapporté (Cat., p. I6r) an Plat. clu Japon, Schl.

Maintenant, après l'énumération des espéces inconnues au Musée de Paris, 
ORDRE DES SALRIENS. - GECKOTJEXS.

au nombre de quinze, et dont la description ne se trouve pas dans l'Erpét. génér., je dois faire connaître une espèce inédite, Hém. atèle, Hem. ateles, A. Dum., et décrire, en outre, l'espèce suivante dont j'ai indiqué les caractères principanx dans le Catalogue, où elle est nommée Hém. taches-rousses.

Celle-ci, en raison de la conformation des pouces inonguiculés, élargis sous tonte lenr longueur et sans phalanges saillantes au-dessus du disque, appartient au genre nommé par Wiegmann Peropus. Parmi les trois sousgenres que M. Fitzinger y a établis, c'est à celui qu'il nomme Peropus proprement dit qu'il faut rapporter cette espèce, car la diagnose suivante donnée par le zoologiste de Vienne pour l'Hém. mutilé, le seul qui, jusqu'alors, dût prendre place dans cette subdivision, convient également bien à ce nouveau Geckotien, comme on en a la preuve par les termes suivants empruntés au Syst. Rept., p. ro3 : Disci scansorii lamellis sulco longitudinali bi-partitis. Plica lateralis distincta, tenuis. Cauda depressa, denticulata. Palma fisse, plante subpalmatce.

IV. - 4 bis. Hémidactyle taches-rousses, Hem. baliolus, A. Dum.

Idem., A. Dum., Cat. du Mus. de Paris, p. 38. - Id., Répert. Erpét. génér., Dum., Bib., t. IX, p. 200 .

$$
\text { (Pl. xvil, fig. 2.) }
$$

Dos couvert de granulations sphériques plus petites sur la région rachidienne que partout ailleurs; plaque rostrale en fer à cheval, contigué par ses extrémités avec les plaques nasales.

Il faut noter, entre autres, comme caractères propres à distinguer du $m u$ tilé l'Hém. taches-rousses, que celui-ci a la tête plus conique, et comme elle n'est pas renflée en arrière des yeux, elle n'est pas aussi distincte du cou. Les grandes plaques sous-maxillaires qui bordent les labiales inférieures, sont moins longues (par erreur typographique, notre Cat., p. 38, dit à ce sujet plus, au lieu de moins). La lèvre supérieure porte, de chaque côté de la mentonnière, huit plaques seulement et non douze, comme celle du mutilé, dont les granulations sur la tête et sur le dos sont un peu plus fines et plus serrées, moins distinctement sphériques, et ne sont pas disposées aussi régulièrement que dans cette nouvelle espèce en anneaux à la face supérieure de la quene. Il y a d'ailleurs, ¿̀ la région sous-caudale, comme chez la plupart

1. Ce rnot est employé par Plaute dans le sens de bai, roux, tacheté. 
des Hémidactyles, de grandes plaques analogues aux ventrales ou gastrostèges et aux urostèges des Ophidiens.

Le système de coloration (fig. 2 de la pl. xvil), consiste en taches rousses ou d'un brun clair sur une teinte brune générale plus foncée. - Le type de cette espèce est unique. Le Muséum l'a reçu de la Nouvelle-Guinée.

\section{V. - 15 bis. Hémidactyle atèle 1, Hem. ateles, A. Dum. ESPÈCE NOUVELLE.}

(Pl. хуні, fig. 9, montrant la main vae par dessous.)

Mains à pouces tronqués et tout à fait mudimentaines, à doigts palmés à leur base, ainsi que les orteils; tete conique, recouverte en dessus et surtout au-devant des yeux, de gramulations plus volumineuses que celles du tronc. Queue déprimée, à bords finement dentelés, et dont les granulations semblables en dessus et en dessous, sont disposées en anneaux réguliers.

La tête est conique et confondue avec le cou; les yeux ont un volume médiocre. Il y a douze plaques à la lèvre supérieure, de chaque côté de la rostrale, qui est plus haute que large et remonte sur le museau, dont l'extrémité est un peu effilée. Les narines sont grandes et presque terminales. La plaque mentonnière, est étroite et triangulaire. On voit sous le menton des scutelles plus volumineuses que les granulations de la gorge, et dont quelques-unes sont régulièrement disposées en ligne le long de la rangée des plaques sous-labiales.

La face inférieure de la queue, contrairement à ce qui a lien chez la plupart des Hémidactyles, n'est pas revêtue de grandes lames transversales. - Il n'y a pas de pores fémoraux.

Le système de coloration a complétement disparu avec l'épiderme. La teinte générale est un gris brunâtre uniforme, qui n'offre une nuance plus tranchée que sur la queue, dont les bords, en dessus comme en dessous, sont d'un brun assez vif. Aucune tache ni bande ne se voient sur les régions supérieures ou inférieures.

4. Ce nom, employé par Geoffroy Saint-Hilaire (Ann. du Mus., 't. VII, p. 260), pour désigner un genre de singes privés de pouce aux mains et non aux pieds (ảrehns, qui est privé, incomplet), 'sert ici à rappeler une disposition analogue, jusqu'à présent unique parmi les Hémidactyles, et qui aurait pu motiver la formation d'un sous-genre. C'est à la suite de toutes les espèces que celle-ci doit prendre place, car elle ne peut rentrer, en raison de la conformation spéciale des mains, ni dans Ie genre Peropus, Wiegm., où le pouce se voit, mais manque de phalanges libres, ni moins encore dans les autres groupes où le pouce est semblable aux autres doigts. 
La longueur totale est de $0^{\mathrm{m}}$ I 5 . La queue entre dans ces dimensions pour $0^{\mathrm{m}} \mathrm{O} 7$, et le tronc el la tête pour $\mathrm{o}^{\mathrm{mm}} \mathrm{o} 8$.

Le caractère tout à fait spécial des pouces rudimentaires de ce Geckotien ne permettant aucune confusion, il est inutile d'insister sur les autres différences qui l'éloignent des divers Hémidactyles décrits jusqu'à ce jour.

Le type de cette espèce nouvelle est un spécimen unique rapporté des environs de Samboangan, petite ville de l'ile Magindanao (Philippines), par MII. Hombron et Jacquinot, chirurgiens des navires $l$ Astrolabe et la Ziélée, durant l'expédition au pôle sud commandée par Dumont d'Urville.

- L'espèce que Wiegmann a nommée H. mutilé, Hem mutilatus, n'était pas connue au Mnsée de Paris, à l'époque où le t. III de l'Erpét. génér. fut publié. On a reçu plus tard, par les soins de madame Marchal, plusieurs individus appartenant à cette espèce et recueillis à l'île de France.

On a pu constater sur ces animaux l'exactitude de la description donnée par le zoologiste de Berlin, et de plus, on a vu qu'il y a, chez les mâles, des pores fémoraux et pré-anaux disposés sur une ligne continue, en forme d'accolade et au nombre de trente-six à trente-huit. Les narines sont séparées par deux petites plaques quadrilatères. La mentonnière large et triangulaire, est contiguë en arrière à six plaques, dont les deux médianes sont allongées.

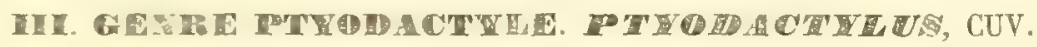

(Pl. xvrr, fig. 10 et $10 a$, la main du ptyodact. frangé.)

Ie nom générique proposé par Cuvier pour trois espèces, le Gecko des maisons ou d'Hasselquist, le G. frangé et la Salamandre aquatique et noire du père Fenillée ou Lacerta caudiverber'a, Linn., a maintenant dans les classifications de plusienrs zoologistes une acception beaucoup moins restreinte. Ainsi, M. Fitzinger groupe dans une même famille, celle des Ptyodactyliens, non-seulement les espèces que Cuvier nommait ainsi, mais de plus, celles que ce demier a réunies dans le genre Sphériod., et il y joint, en outre, les types des genres Phyllodactyle et Diplodactyle de M. Gray.

On comprend, en effet, ce rapprochement, en raison de la conformation des doigts qui, malgré des différences notables, suffisantes pour motiver des subdivisions, offrent cependant cette analogie qu'ils sont dilatés seulement à leur extrémité libre. Il est fâcheux néanmoins que le mot Pyodact., qui 
signifie doigt en éventail, serve en outre à désigner des animaux dont les disques sous-digitaux ont une tout antre disposition. Cet inconvénient, au reste, est moindre que ne le serait celui de l'emploi d'un nom nouveau.

Les subdivisions de la famille ou les genres sont, dans le Syst. de M. Fitzinger, au nombre de six. (Voy. plus haut le tableau de la Fam. II, p. 441). 11 y en a deux (Ptrodact., Cuv., et Oiacurus, Fitz.), qui se rapportent spécialement au groupe des Ptyodactyles proprement dits ou Geckos munis de deux séries de lames en éventail sous l'ex trémité des doigts.

N. Gray (Cat.) distribue ceux-ci dans les trois genres Ptyodactyle, Cuv., Caudiverbera, Laur., et Uroplate, Dum. (Voy. plus haut le tableau, p. 448.)

Ce dernier genre proposé par mon père (Zool. analytique, p. 81, I 8o5) comprenait alors toutes les espèces qu'on nommait auparavant les Geckos is queue plate, mais il a été ensuite fort restreint par M. Fitzinger (Neue Classificat. der Rept., I 826 , p. 46), qui n'y rapportait que le Stellio fimbriatus, Schn., lequel, devenu maintenant pour lui, à l'exemple de Wagler, Rhacoèssa fimbriatus (Syst., p. 97), est encore un Uroplate dans la classification de M. Gray, ainsi que le Ptyod. rayé, Dum. et Bib.

Aucune nonvelle espèce de Ptyod. proprement dits n'a été reçue au Musée de. Paris depuis i 836, ou n’a été décrite par les zoologistes.

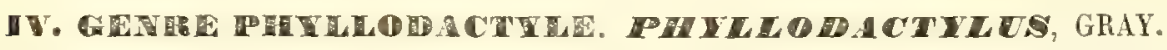

(Pl. xviI, fig. 14 et $11 \alpha$, main et doigt du Phyll. porphyré.)

La division principale qui ait été faite dans ce genre caractérisé par la présence à l'extrémité des doigts de deux disques simples que sépare un sillon qui reçoit l'ongle, est fondée sur l'apparence un peu différente de ces disques. Dans les vrais Phyllodactyles, Gr., ils sont planes, tandis qu'ils sont convexes dans les Diplodactyles, Gr. Cette différence n'est cependant pas tellement apparente, qu'il soit toujours très-facile de la constater.

Aux deux espèces types: Dipl.vitlatus, Gr., et Dipl.gerrhopygus, Wiegm., le zoologiste anglais en ajoute, dans son Cat. (p. 149), quatre nouvelles, qui sont inconnues au Musée de Paris. Il y en a trois de l'Australie : Dipl. ornatus, ocellatus, bilineatus, et la quatrième, Dipl. lineatus, recueillie au Cap de Bonne-Espérance, est décrite, en outre, par M. Smith (Illustr. of the Zool. of S. $A f r$. Appendix, p. 6). Nous ne connaissons pas non plus le Dipl. lepidopy- 
gus, Tschudi, Conspectus Repl. que in republ. Peruana reperiuntur (Erichson's Archiv. I $1^{\circ}$ année, t. I, Berlin, 1845 , et dans la Faune, p. 38), ni Dipl. piclus, Peters, de Madagascar, loc. cit., p. 615.

De plus, M. Gray admet, sous le nom de Strophura, un genre particulier, dont le type est une espèce décrite d'abord dans l'Erpét. génér., Phyll. strophurus, Dum., Bib., qu'il confond, mais à tort je pense, comme je cherche à le montrer plus loin, avec le Gecko nommé par lui Stroph. spinigera, Gr.

Enfin, il rapporte avec doute, il est vrai, le Phyll. de Lesueur, Dum., Bib., à une autre division du même groupe, au genre $\mathbb{E}$ dura, Gr., dont le type est Edura marmorata que nos collections ne possèdent pas.

M. Fitzinger (Voy, tabl. de la Fum. II, p. 441), n'admet que trois genres :

$I^{\circ}$ Diploductylus, Gr., comprenant deux sous-genres : a. Pachyurus Lesueuri (Phyll., Les., Dum., Bib.). - b. Dipl.vittatus, Gr., et Dipl. gerrhopygus, Wiegm.

$2^{\circ}$ Phyllodactylus à écaillure homogène : deux sous-genres : a. Phyll. porphyreus, Wiegm. (Afr.), et Phyll. Peronii, Fitz. (Australie) séparé du précédent sans autre motif que la différence d'origine; puis Phyll. gymnopygus, Dum., Bib.; - b. Euleptes Wagleri, Fitz. (Phyll. europaus, Gené).

$3^{\circ}$ Discodactylus à écaillure hétérogène ou entremêlée de tubercules : deux sous-genres : a. Disc. pulcher, Fitz. (Phyll. pulcher, Gr.); Discod. tuberculosus, Fitz. (Phyll. iuberc., Wiegm.). - Ici vient se placer une espèce du Pérou, Disc. phacophorus, Tschudi, fauna Peruana (p. 38), qui ne fait pas partie des richesses de notre Musée. - b. Strophurus Dumerilii, Fitz. (Phrllod. stroph., Dum., Bib. et non Gymn. stroph., comme le porte, par erreur, la liste insérée plus haut p. 444).

VI. - 2 bis. Phyllodactyle D'Europe, Phyll. europceus, Gené.

Gené, Synopsis Rept. Sardinix, p. 9, tab. I, fig. A. - Id., Bonap., Amph. europar, p. 29, et Iconogr. del Fauna italica, texte sans pagination et planche sans ${ }^{\circ}$, fig. 1 , vu en dessus et en dessous, - Phyllodactylus (Euleptes) Wagleri, Fitz., Syst. Rept., p. 95. Dans cet ouvrage, l'auteur cite, comme dénominations antérieures à celle que Gené a proposée: Ptyodactylus caudivolvulus, Fitz. Gravenhorst, Mus. de Breslau, et Euleptes Wagleri, Fitz. - Phyll.europæus, Dum., Cat. des Rept. du Hus. de Paris, p. 41. - Id., Répert. Erp. gén., Dum., Bib., t. IX, p. 25 z.

Doigts d disques terminaux plats, et à lames non divisées; granulations des téguments égales entre elles; corps déprimé; queue un peu aplatie, fusiforme, rétrécie à sa base, portant, de chaque coté, au-dessus de ce point, un tubercule saillant. 
La tête est déprimée, légèrement efflée en avant et élargie en arrière, ce qui fait paraitre le cou un peu plus étroit. La plaque rostrale est grande et largement rabattue sur le museau où elle est en contact, de chaque côté, avec une grande plaque nasale, qui borde en haut la narine, dont l'ouverture est circonscrite en avant et en bas, un peu par la rostrale et surtout par la première labiale supérieure. Le cercle squameux est complété en arrière par deux petites plaques. A la lèvre supérieure, il y en a onze ou douze.

Les granulations du dos sont presque circulaires, petites et égales entre elles. Les squames des régions sous-maxillaire et gulaire sont beaucoup plus petites que celles de l'abdomen.

Les régions supérieures ont une teinte carnée assombrie par un gris cendré qui la recouvre en grande partie, de sorte que le fond ne se voít que sous l'apparence d'une multitude de petits points plus clairs et de courtes bandes transversales sur la région médiane du dos. En dessous, aucune tache ne se voit, si ce n'est sous la queue, dont les bords portent chacun une série de pelites maculatures foncées, régulières.

Nos trois exemplaires recueillis en Sardaigne et donnés au Muséum par M. Gené et par S. A. le prince Ch. Bonaparte, sont de petite taille comme ceux qui ont été figurés par ces zoologistes.

Le plus grand est long de $a^{\mathrm{m}} 07^{5}$ ainsi répartis : tête et tronc, $\mathrm{o}^{\mathrm{m}} \mathrm{o}$. $\mathrm{O}$, queue, $\mathrm{o}^{\mathrm{n}_{1}} \mathrm{o} 35$.

Ce Phyllodactyle, avec le Plat. des murailles et l'Hém. verruculeux, constitue la troisième espèce européenne de Geckotiens, mais tandis que ces denx dernières espèces ont une zone d'habitation assez étendue, car on les trouve sur toutes les côtes de la mer Méditerranée, celie dont il s'agit paraît, selon la remarque du prince $\mathrm{Ch}$. Bonaparte, ne vivre que dans la Sardaigne où elle se rencontre assez abondamment sous les écorces, dit M. Gené, et plus rarement sous les pierres. C'est, du reste, une espèce nocturne, à pupille verticale, comme celle d'un grand nombre de Geckos.

Il est facile de reconnaître que ce Phyllodactyle, outre la différence si importante d'origine, puisque nul de ses congénères n'habite l'Europe, se distingue de ceux-ci par certaines particularités. D'abord, il ne peut être confondu, en raison de la conformation de ses disques sous-digitaux, avec aucune des espèces réunies dans le groupe des Diplodacíyles.

D'autre part, si la comparaison est établie avec les Phyllodactyles, qui ont, 
ORDRE DES SAURIENS. - GECKOTIENS.

comme lui, des disques plats, l'intégrité des lames sous-digitales ni divisées, ni échancrées en chevron, l'absence d'une surface nue au-devant du cloaque, ou de tubercules entremêlés aux granulations des téguments, l'impossibilité où il est d'enrouler la queue, et enfin le système de coloration, en même temps que sa petite taille, ne permettent aucune confusion avec les Phyll. de Lesueur, g7mnopyge, tuberculeux, gentil, strophure et porpyhré.

VII. - 6 bis. Phyr.todactrle spinigène, Phyll. spinigerus, Dum.

Diplodactylus spinigerus, Gr., Zool. misc., p. 53. - Strophura spinigera, Gray, Zoal. Erebus and Terror et Cat. of Liz., p. 148. - Phyll. spinigerus, Dum., Cat. des Rept. du Mus. de Paris, p. 41. - Ilem., Répert. Erpét. génér., Dum., Bib., t. IX, p. 252.

De chaque cóté du dos et de la queue, une série de tubercules pointus et constituant à la région. caudale deux nangs parallèles de longues épines.

La tète est assez large en arrière; le museau est un peu court. La queue est légèrement recourbée en dessous. L'écaillure du tronc et de la queue n'offre d'autre irrégularité que celle qui résulte de la présence, au milieu des granulations arrondies, de tubercules coniques peu apparents sur le dos, mais très-saillants et transformés sur la région caudale, où ils sont régulièrement espacés, en véritables épines coniques et fort pointues, longues de $\mathrm{o}^{\mathrm{m}} \mathbf{0 o z}$.

Ce caractère seul sufft pour empêcher la confusion de ce Geckotien avec tout autre reptile de la même famille. Aussi, ne semble-t-il pas possible de le considérer, ainsi que M. Gray le fait dans son Catulogue, comme identique avec l'espèce que mon père et Bibron ont décrite sous le nom de Pliyllod. strophure.

Ce dernier, par un fâcheux hasard, ne se trouve plus dans les collections du Muséum, et comme ce type était unique, il n'est plus possible de le soumettre avec le Phyll. spinigère à un examen comparatif permettant une indication précise des analogies et des différences. Si cependant ou s'en tient aux termes mêmes de la description de l'Erpét. génér. et au dessin donné sur la pl. xxxir de cet ouvrage, fig r, i $a$ et i $b$, on voit des différences assez marquées pour y reconnaître une espèce autre que celle que je décris ici d'après M. Gray. Je ne m'étendrai pas davantage sur cette nécessité de maintenir comme distinct le spinigère, car l'apparence singuliere et tout à fait 
identique de cette queue épineuse chez les cinq individus de nos collections, ne peut laisser aucun doute à cet égard.

La plaque rostrale est divisée longitudinalement sur la ligne médiane, de sorte qu'elle a, dans son entier, la forme de deux pentagones placés l'un à côté de l'autre. La narine est circonscrite par six écailles, la rostrale, la première labiale, deux nasales supérieures, et en arrière par deux petites écailles quelquefois réunies en une seule. On compte quatorze à quinze plaques labiales supérieures, de chaque côté de la double rostrale.

Les plaques sous-digitales qui précèdent les disques sont échancrées en chevrons sur les trois doigts du milieu; elles sont simplement transversales au premier et au cinquième doigts.

Contrairement à l'assertion de M. Gray, il n'y a pas de pores anaux, du moins sur nos échantillons.

La teinte générale, à en juger par les points où l'épiderme n'a pas été détruit, est un vert-olive finement piqueté de points noirs. Sur la région dorsale moyenne, ces points sont plus apparents et plus rapprochés les uns des autres; il en résulte une série de taches ovalaires, qui réunies les unes aux autres par leurs extrémités, représentent une sorte de large bande festonnée. La région inférieure est blanchâtre et sans taches.

Les dimensions de l'un des individus qui diffèrent à peine entre eux sont de $\mathrm{o}^{\mathrm{m}} \mathrm{r}_{02}$ ainsi répartis : tête et tronc, $\mathrm{o}^{\mathrm{m}} \mathrm{o} 6 \mathrm{~g}$, queue, $\mathrm{o}^{\mathrm{mm}} \mathrm{o} / \mathrm{l} 2$.

Le Muséum a reçu cette espèce par les soins de M. J. Verreaux, qui en a pris cinq exemplaires dans la province de la rivière des Cygnes, en Australie.

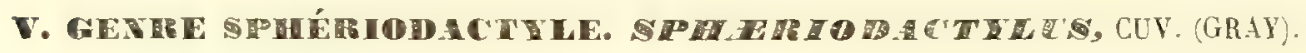

Pl. xvir, fig. 12, $12 a$ et $12 b$, main du Sph. très-petits points, et doigt vu en dessus et en dessous.

Ce genre, dont le nom se trouve reproduit maintenant par la plupart des naturalistes avec le changement que lui a fait subir à tort Wagler, en le nonmant Sphcerodactylus, est, dans la famille des Geckotiens, le groupe qui a le moins été modifé depuis l'époque où M. Gray en a retiré comme types des Phyllodactyles, les deux espèces munies d'ongles que Cuvier y avait introduites. L'histoire de deux espèces ( $S p h$. sputator et punctatissimus), a été complétée d'une manière fort intéressante, en 1843, par Cocteau dans la description des Reptiles qu'il a faite pour l'ourrage publié par M. Ramon de 
ORDRE DES SAURIENS. - GECKOTIENS.

la Sagra, sous le titre de : Hist. phys, polit. et natur. de l'lle de Cuba ${ }^{1}$ Rept., p. Ico-I 73 , pl. xvir et xurr.

Les espèces qui manquent dans nos collections et que l'on trouve décrites dans les publications postérieures à l'année 1836 , sont les suivantes: Sphor. nigro-punctalus, Gr., et Sphar. Richardsonii, Gr., distinct du précédent et de ceux que l'on connaissait déjà par la présence de séries transversales d'écailles rhomboïdales et carénées. Ces différents types proviennent de l'Amérique du Sud ou des Antilles. C'est à la Jamaïque, en particulier, que M. Gosse a recueilli les Sphcer. argus et oxyrhinus, dont il ne fait connaître que le système de coloration et que le Musée de Paris ne possède pas ( $A n n$. of nat. hist., $2^{\circ}$ série, r 850 , t. VI, p. 347).

Nous avons reçu dans ces dernières années quelques nouveaux animaux appartenant au genre dont il s'agit, mais nous avons hésité à les considérer comme types d'espèces nouvelles, car il n'y a guère que le système de colcration qui offre certaines dissemblances. $1^{\circ}$ Tels sont, par exemple, 2 petits individus rapportés de Cuba par M. Morelet, qui en a généreusement fait don au Muséum avec beaucoup d'antres reptiles intéressants recueillis par lui pendant son voyage dans cette île et dans l'Amérique centrale. On ne trouve, en effet, aucune différence spécifique réelle avec le Sphér. à très-petits points, mais, comme je l'ai dit (Ca/., p. 42), le pointillé blanc y est à peine apparent, et de plus, on voit, d'une façon peu distincte, sur la nuque, à la base de la queue et sur un ou deux points de sa longueur, de petites taches noires.

$2^{\circ}$ Relativement au Sphér. bizarre (pl. xvir, fig. 3, 3 a et $3 b$ ), les auteurs de l'Erpét. génér. ont été embarrassés pour des sujets qui ne présentaient pas le singulier système de coloration des types étudiés par Cuvier. Ils n'ont pu cependant les décrire que comme variété à bandes blondes à cause de la teinte de larges raies longitudinales régulièrement disposées sur la tête et se réunissant en angle sur la nuque (Voy. pl. xvir, fig. $3 c$ ).

3॰ J'ai également éprouvé un assez grand embarras pour deux sphériod. recueillis dans l'île Sainte-Lucie par M. de Bonnecour. Par tout l'ensemble de leurs caractères, ils offrent une grande analogie avec le Sphér. bizarre (pl. xvir, fig. 4 et $4 a$ ). Leur système de coloration cependant n'est pas le

1. Cette intéressante Erpétologie est l'œuvre de Cocteau pour les Chéloniens et pour les Sauriens. Sa mort prématurée ne lui a pas permis d'achever cette cuvre, dont la fin à été confiée à Bibron, qui derait lui-même, et peư d'années après, succomber avant l'âge.

Archives du Muséus. T. VIII. 
même, car ils n'ont pas cet aspect singulier du Sph. bizarre qui résulte de la présence de vermiculations blanches se détachant sur la teinte brun-foncé de la tête, laquelle établit un contraste frappant avec la nuance beaucoup plus claire du tronc. Ici, la tête a la même couleur que le reste du corps. Sous la gorge, il y a quatre bandes brunes disposées en chevrons emboîtés et à sommet postérieur. Dans l'intervalle que laissent entre elles, en avant, les branches du plus fort chevron, on voit des taches brunes peu volumineuses. Une bande plus foncée part de l'oeil et se dirige en arrière et en dedans, parallèlement à la branche correspondante du plus grand chevron. Il naît, en outre, du bord postérieur de chaque oeil, mais an-dessus de la précédente, mne autre bande noire; après un court trajet horizontal, elle s'infléchit en dedans et va rejoindre sur la nuque la bande du côté opposé, de manière à former avec elle une sorte de couronne qui circonscrit la face supérieure de la tête. Un large demi-collier noir complète le système de coloration des régions antérieures parfaitement identique sur nos deux exemplaires.

A ces différences qui pourraient ne caractériser qu'une variété (ITnTiété da laches noires, Cat. du Mus. de Paris, p. 42), il faut en joindre qui ont plus d'importance, mais qui ne sont pas tellement tranchées qu'elles puissent être considérées comme vraiment spécifiques. Je veux parler des écailles, qui sont ici un peu plus petites, pent-être moins régulièrement disposées et de l'aspect de celles de la région rachidienne, qui forment une bande moins nettement distincte des antres granulations plus grandes au milien desquelles elles sont placées, et d'ailleurs la peau forme, le long de la colonne vertébrale, un pli plutôt qu'un vrai sillon.

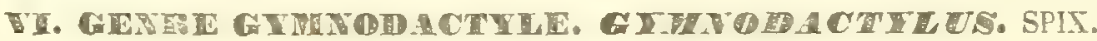

P1. IVII, fig, $13,13 a$ et 1 '́

L'absence d'un disque sous les doigts est, chez certains Geckos, un caractère fort important. Il semblerait, au premier abord, devoir motiver leur réunion en un groupe tout à fait spécial et qui comprendrait, d'une part, toutes les espèces que, sous des noms génériques différents, les naturalistes ont rapprochés du Gymnodactylus Geckoides, Spix, et d'autre part, les Sténodnctyles de Cuvier. C'est par ce motif que M. Fitzinger considère tous ces Geckotiens comme appartenant à une seule et même famille: Stenodactyli 
ORDRE DES SAURIENS. - GECKOTIENS.

(Voy. le tabl. de la Fam. I, p. 441). - Pour lui, les vrais Sténodactyles ou Geckos à doigts granuleux en dessous et dentelés sur les bords constituent, dans cette famille, deux genres distincts. - Les antres, au contraire, en forment quatre d'après la conformation des doigts et le mode d'articulation des phalanges entre elles.

A ce point de vue, et suivant l'aspect de l'écaillure, selon qu'elle est simple ou bien hétérogène, on a multiplié les coupes génériques.

Si, par ordre de dates, nous passons d'abord en revue la classification de II. Fitzinger, nous voyons, en laissant de côté les vrais Sténodactyles, que les antres Geckotiens sans disques et à doigts étroits, offrent cette particularité notable que le cinquième orteil est dirigé en dehor's, et, comme on le dit, versatile. Les doigts n'ont pas toujours la même conformation.

A. Les doigts sont-ils droits? Alors, $I^{\circ}$ quand l'écaillure est composée de granulations entremêlées de tubercules, ce sont des Gymnodactyles divisés en 2 sous-genres, selon que la queue est déprimée (Anomalurus: Gymn. Miliusii, D. B.) ou arrondie (Gymodactylus proprement dit : G. fusciatus et Dorbignii, D. B.). - $2^{\circ}$ Lorsqu'il n’y a point de tubercules cutanés, ces Geckotiens ì doigts rectilignes sont réunis dans le genre Saurodactylus, subdivisé lui-mème en Pristiurus, Rüppell, pour le seul Geckotien à crête suŁ le dos et. sur la queue: G. flavipunctatus, D. B., et en Saurodactylus, Fitz., proprement dit, pour les espéces sans crête : a. G. mauritanicus, D. B.., dont MI. Fitzinger sépare les sirjets du Musée de Vienne originaires de l'Afrique centrale, sous le nom de Saurodact. desertorum, Fitz; b. G. Timoriensis, D. B., et c. G. Gaudichaudii, D. B.

B. Si les doigts, au lieu d'être droits, sont anguleux et paraissent comme I)risés au niveau de leurs articulations, il fautencore, pour ce second groupe, tenir compte de l'écaillure, car M. Fitzinger range ceux qui n'ont pas de tubercules dans le genre Gonalodes,. Fitz.: (Grmn. albigularis, D. B.), et dans le genre Gonyodactylus, Fitz., les espèces à granulation hétérogène qu'il rapporte à cinq sous-genres : r. Phyllurus, Cuv. : (Stellio phyllurus, Schneider); 2. Dasyderma, Fitz. : (Gymn. geckoides, Spix); 3. Gonyodactylus proprement dit, Kuhl: (Gonyodact. marmoralus, K.); 4. Cyrtopodion, Fitz. : (Stenodacl. scaber, Rüpp., dont M. Fitzinger sépare, sous le nom de Gonyodact. cyprius, des exemplaires reçus au. Musée de Vienne de l'Asie et de l'île de Chypre), et 5.Cyrtodactylus, Gr. :(Cyrt. pulchellus,.Gr.). 
-M. Gray, outre les genres Pristiurus, Rüpp., Gonyodacl., Kuhl, Gymnodactylus, Spix, et Phyllurus, Cuv., conserve dans son Catal. les genres Cyrtodactylus et Naultinus qu'il avait précédemment proposés, et de plus, en établit trois nouveaux dans cet ouvrage : Cubina: (Gymn. fasciatus et G. Dorbignii, D. B.), Homonota : (G. Gaudichaudii, D. B.), et Heteronoti. Voy. le tableau de la Fam., d'après le système de M. Gray, p. 448.

- Plusieurs espèces que le Musée de Paris ne possède pas ont été décrites dans ces dernières années. Il y en a deux, en particulier, au Musée de Breslau, que M. Gravenhorst avait nommées, l'une Stenodact. brachypus, l'autre Gymnodact. lavis; M. Fitzinger les place dans le genre Gonatodes en laissant à cette dernière son nom spécifique, et en échangeant pour la première la dénomination de brachypus, contre le nom du célèbre naturaliste de Breslau : Gonatodes Gravenhorstii, Fitz.

Dans le Catalogue de M. Gray, nous trouvons les Gymnodactyles dont les noms suivent, et qu'il a le premier fait connaitre : Naultinus punctatus, Gr. in Dieffenb. N. Zel.; Gonyodact. australis, Gr. (Terr. et Erebus); Gonyod.? (Cyrtodact.) ocellalus, Gr. (Zool. miscell.); Heteronota Kendallii, Gr. (Cal.), Heter. Binvei, Gr. (T'err, et Erebus); Phyllurus inermis, Gr. (Terr. et Erebus). Plus récemment, ce zoologiste a mentionné une nouvelle espèce: Gonyodact. indicus, Gr. (Annals of nat. hist., t. XVIII, p. 429). Le Musée ne la possède pas; il en est de même pour deux autres : Naultinus Grayiu Bell (Zool. of the Beagle) et (rymnodact. Kandianus, Kelaart (Prodromus fauna zeylanice) .

Je me borne à ces simples citations pour les différentes espèces que je viens d'énumérer, mais il en est trois autres peu connues, qui se trouvent dans nos collections, et dont je dois parler avec quelques détails : ce sont les Gymnodactyles élégant, Gr., caspien, Eichw., et celui que M. Gray a nommé Coléonyx élégant, qui est décrit dans le Cat. du Mus. de Paris sous les noms de Gymn. à scapulaire. Je dois enfin donner la description d'une espèce signalée dans cet ouvrage où je l'ai fait connaître pour la première fois : Gymnodact. d'Arnoux, et de trois autres inédites jusqu'à ce jour : Gymn. humeralis, Guich., varius, A. Dum., et Persicus, A. Dum., auxquelles il faut joindre le Stenodactylus (Gymnod.) fuscus, Hallowell.

De plus, j'appelle ici l'attention sur une variété remarquable du Gymn. albogularis, Dum., Bib., dont un spécimen placé depuis peu de temps dans 
l'alcool et appartenant à une collection particulière, a été vu par M. Séraphin Braconnier, attaché au laboratoire d'Erpétologie et d'Ichthyologie au Muséum, et à qui je dois de bonnes observations sur les Reptiles, dont il est question dans ce mémoire. La description qui suit fait mieux connaître la vivacité des teintes en partie altérée sur nos types: Le cou est d'un jaune orangé; on y voit deux lignes blanches en chevron, prolongées jusqu'aux plaques labiales inférieures. Une autre ligne blanche médiane, sous le menton, emboîte la plaque mentonnière, et les écailles qui la suivent. La lèvre supérieure porte, au-dessous de l'œil, une tache blanche. La queue, dans son dernier tiers, est jaun âtre.

- Tous les Geckos à doigts sans disques et distincts des Sténodactyles par l'absence de granulations à leur face inférieure, ainsi que de dentelures latérales, conservent ici le nom générique de Gymnodactyles proposé par Spix.

Quelques espèces cependant présentent des particularités assez notables pour qu'elles puissent être considérées comme types de sous-genres.

On pourrait, en effet, $1^{\circ}$ à l'exemple de Cuvier, nommer Phyllure le Gecko signalé d'abord par White sous les noms de Lacerta platura, puis de Stellio phyllurus, par Schneider; $2^{\circ}$ comme l'a proposé M. Rüppel, se servir du nom de Pristiure pour l'animal qui a reçu, dans sa Faune d'Abyssinie, la dénomination spécifique de flavipunctatus. $3^{\circ}$ Par les mèmes motifs, il y aurait peut-être certains avantages à ne pas rejeter le mot Naultinus, sans élymologie connue, employé par M. Gray pour désigner plusieurs Gymnodactyles à doigts plus élargis qu'ils ne le sont d'ordinaire, et parmi lesquels se trouve, avec de vrais Gymnodactyles, le Gecko dont j’ai précédemment parlé (Platyd.pacificus, Dum., Nault. pacif., Gr.). Ce nom serait réservé pour les espèces semblables au Gymn. élégant par la forme de leurs doigts rectilignes et à larges lamelles inférieures. $4^{\circ}$ Enfin, la disposition des ongles et de leur gaîne protectrice est assez remarquable dans l'espèce type du genre Coléony $x$ du zoologiste anglais, pour que j’aie cru devoir conserver comme dénomination spécifique ce mot tiré du grec (

-En suivant l'ordre indiqué par les affinités mutuelles de ces Geckotiens et adopté par mon père et par Bibron pour leurs descriptions, je dois mentionner d'abord des Gymnodactyles donnés par M. le comte de Castelnaw, et que j'ai signalés dans le Cat. du Mus. de Paris, p. 43, comme appartenant à l'espéce dite Gymnodact. de Gaudichaud. 
Un nouvel examen de ces Sauriens montre que les différences sur lesquelles j'avais appelé l'attention, sont assez importantes pour qu'il devienne nécessaire de les séparer du type auquel je les avais primitivement rapportés. Ils représentent une espèce distincte et différente de toutes celles qui ont été décrites jusqu'à présent dans le genre dont il s'agit.

M. Guichenot l'a fait connaître et figurer dans l'Erpétologie du Voyage de M. de Castelnau (p. 13, pl. III, fig. I, $a$ et $b$ ), sous le nom de Gymnodact. huméral qu'il a choisi pour la désigner.

VIII. - 2 bis. Gymrodactyle humérar, Gymnodactylus humeralis, Guich.

Granulations des régions supérieures très-fines et très-serrées, non entremélées de tubercules; tête assez épaisse, peu large; museau court, déclive; de gros points noîs sur les flancs; audevant de chaque épaule, une bande jaune verticale bordée de noir.

Les formes sont assez élancées; la queue peu robuste est plutôt allongée.

Les granulations sont plus volumineuses sur la tête que sur le dos, mais les plus fines sont celles de la région gulaire qui, par cela même, diffère notablement de l'abdomen dont l'écaillure se compose de petites squamęs faiblement imbriquées, à bord postérieur arrondi.

La plaque rostrale est en contact, par son bord supérieur, avec les plaques nasales qui sont séparées, sur la ligne médiane, par une ou deux granulations plus grosses que celles qui les suivent. De chaque côté de la rostrale, on compte sept plaques sus-labiales. La mentonnière est grande et représente un triangle à sommet postérieur tronqué; elle est suivie, de chaque côté, d'une rangée de plaques labiales diminuant graduellement de dimensions, et dont la première a plus de longueur que de hauteur.

Il n'y a point de pores fémoraux. La pupille est ronde. Le système de coloration est fort altéré, mais nous trouvons, dans un croquis de M. de Castelnau et dans ses notes, de précieux renseignements; c'est en puisant à celte double source que la planche III, fig. I de l'Erpétologie de son Voyage a pu être coloriée.

Voici l'indication même donnée par M. de Castelnau : « Tête pourpre, avec des taches d'un beau bleu d'azur très-clair; le corps d'un jaune verdâtre, couvert de petites piquetures noires; le dos un peu brun; une dizaine 
de taches noires de chaque côté du tronc; queue d'un brun obscur, avec des taches transversales plus sombres; au-devant du membre antérieur, une ligne jaune, oblique, un peu sinueuse, étroite et bordée de noir; gorge d'un beau jaune éclatant; le ventre et le dessous des pattes et de la queue d'un gris brun nuancé de violet; l'iris est jaune. »

Ce riche système de coloration sur lequel nous ne possédions pas ces indications à l'époque où parut la première livraison de notre Catalogue, ne laisse plus de donte sur les différences spécifiques entre ce Gymnodact. et celui de Gaudichaud, où l'on ne trouve aucune trace de ces particularités dont quelques-unes, au contraire, peurent être encore distinguées sur les exemplaires de l'espèce nouvelle.

Il faut noter, d'ailleurs, comme caractères distinctifs du Gymnodact. de Gaudichaud, les dimensions un peu plus considérables des pièces de l'écaillure dorsale qui ressemblent à de petites squames légèrement imbriquées plus qu'à de simples granulations; la forme plus aplatie de la tête, qui est en mème temps plus large; l'étendue moindre de la plaque mentonnière et enfin, l'apparence générale de la queue: elle est en effet plus courte et plus volumineuse que celle du Grmnod. huméral.

Ces nonveaux Geckos sont de petite taille. Le plus grand a une longueur totale de $\mathrm{o}^{\mathrm{m}} \mathrm{O}_{2} 2$ ainsi répartie : tête et tronc, $\mathrm{o}^{\mathrm{m}} \mathrm{0} 3 \mathrm{7}$, queue, $\mathrm{o}^{\mathrm{m}} \mathrm{0} 45$.

MI. de Castelnau et Deville ont rapporté plusieurs échantillons de cette espèce de la mission de Sayaracu, sur les hords de l'Ucayale (Pérou). Elle y est très-commune. M. Tschudi ne l'a pas signalée (Fauna peruana).

IX. - 2 ter. Grunodactyle varué, Gymnodactylus varius, A. Dum.

Granulations des régions supérieures médiocrement fines, non entremélées de tubercules; têle ipaisse, large; musean court, cléclive, à plaque mentonnière très-grande; petites taches noires irrégulières, situées entre d'autres taches claires et se détachant les unes et les autres sur la teinle générale, qui est brune.

I.es formes sont peur élancées et même ces Gymn. sont trapus; leur queue est assez robuste et assez allongée. Les granulations des régions supérieure et gulaire, ainsi que les squames du ventre légèrement imbriquées et à bord postérieur arrondi, n'offrent rien de particulier à noter, si ce n'est qu'elles sont plus grandes que celles du Gymnod. huméral, ce qui établit entre ce dernier et celui dont il s'agit ici, une différence frappante. 
La plaque rostrale très-fortement rabattue sur le museau, est en contact par son bord supérieur avec les plaques nasales que séparent une ou deux grannlations. De chaque côté de la rostrale, on compte six plaques suslabiales. La mentonnière est fort grande; elle emboîte toute l'extrémité de la mâchoire inférieure et son bord postérieur, qui est large et représente le snmmet tronqué de cette plaque triangulaire, est en contact avec deux grandes squames sous-gulaires formant le milieu d'une rangée d'autres écailles dont les dimensions vont diminuant insensiblement de diamètre. Ces plaques sont en contact par leur bord antérieur avec les sous-labiales. Celles-ci sont au nombre de 5; la première est bien plus haute qu'elle n'est large; son bord inférieur, au lieu d'être parallèle au supérieur, comme cela se voit d'ordinaire, esṫ oblique, et il en résulte que c'est en avant, que la hauteur de cette sous-labiale est le plus considérable. La deuxième plaque est identique à la précédente pour la forme, mais un peu moins grande; la troisième est beaucoup plus petite et au-dessous d'elle, il s'en trouve une qui lui est tout à fait semblable; î'une et l'autre ont ensemble une hauteur égale à celle de chacune des deux précédentes; la quatrième et la cinquième sont fort petites.

Il n'y a point de pores fémoraux. La pupille est ronde.

Le système de coloration est bien conservé. Ce qui frappe surtout, c'est une sorte de bigarrure résultant de l'assemblage assez irrégulier sur la tête, sur le dos et sur les membres, de taches d'une teinte claire et de taches noires, Ces dernières cependant forment sur le dos deux séries parallèles séparées par la ligne médiane, qui est d'une teinte moins foncée que les parties environnantes. Sur la tête, il y a des lignes courbes d'une teinte sombre à convexité postérieure. Les flancs sont pointillés de noir et de blanc. Les régions inférieures ont une nuance pâle, et la région gulaire, depuis le bord postérieur des plaques mentonnières jusqu'au niveau de l'angle des mâchoires, est parcourue, d'avant en arrière, par une ligne étroite et blanchâtre.

Les deux espèces auxquelles celle-ci ressemble le plis sont le Gymnod. de Gaudichaud, et surtont le Grmnod. huméral, mais elle se distingue de l'une et de l'autre d'abord par son système de coloration, par l'ensemble de ses formes moins élancées et plıs robustes, puis par l'exagération même des caractères tirés de l'écaillure du tronc, ainsi que de la forme de la plaque 
ORDRE DES SAURIENS, - GEGKOTIENS.

mentonnière, et qui ont déjà servi comme moyens de distinction entre les deux espèces auxquelles je compare ce Gymn. varié.

Il nous est connu par cinq exemplaires en très-bon état de conservation tout à fait semblables entre eux. Leur taille à peu près identique est, pour le plus grand, de $o^{\mathrm{m}}$ o 70 ainsi répartis : téte et tronc, $\mathrm{o}^{\mathrm{m}} \mathrm{o} 35$, queue, $\mathrm{o}^{\mathrm{m}} \mathrm{o} 35$. Ils ont été envoyés de Cayenne au Musée de Paris.

\section{X. - 2 quater. Gymnodactrle Brun, Gymnodactylus fuscus, Dum.}

Stenodactylus fuscus, Hallowell (Journ. of the Acad. of Philadelphia, 185k, p. 33).

Régions supérieures d'une teinle brune tantot foncée, tantot plus claire, avec de nombreuses mouchetures noires; régions inférieures d'un jaune clair; point de pores aux cuisses, ni au cloague; cinq plaques sous-labiales de chaque coté; granulations du dos petites et uniformes.

Les dimensions sont peu considérables, car la tête et le corps ne mesurent pas ensemble plus de I pouce un quart à I pouce et demi (mesure anglaise). Dans notre spécimen donné par M. Hallowell, comme chez ceux que ce zoologiste a examinés, la queue est mutilée. Il en a reçu de nombreux exemplaires de l'État de Nicaragua (Amérique centrale).

La conformation des doigts, qui sont un peu effilés et légèrement anguleux, non dentelés sur leurs bords et garnis en dessous de lames transversales, ne laisse aucun doute sur la détermination de ce Gecko. Ce n'est point un Sténodactyle proprement dit, mais un Gymnodactyle à écaillure homogène, dont la vraie place est à la suite des espèces qui viennent d'être décrites. Il en diffère surtout par son origine. Il est le seul Gymnodactyle qui, jusqu'ici, ait été trouvé dans le continent américain septentrional, mais on voit qu'il appartient à la limite la plus inférieure de cette contrée.

\section{XI. - 5 bis. Grunodactrle élégant, Gymnodactylus elegans, Dum.}

Naultinus elegans, Gr., Zool. miscell., p. 72. - Id., Gr., Dieff. New-Zeal., t. II, p. 203. - Id., Cat. of Liz., p. 169. - Gymn. élégant, Dum., Cat. Mus. de Paris, p. 43. - Id., Id., Répert. Erp. génér., Dum. Bib., t. IX, p. $25 \dot{4}$.

$$
\text { Pl. xviIr, fig. 1k, l'un des doigts vu en dessous. }
$$

Téte épaisse et large en arrière; museau obtus; plaque rostrale dilatée en travers, le plus souvent double; plaques labiales presque constamment au nombre de 12 en haut et de 10 en bas; queue longue, urrondie, portant à sa base de petits tubercules. 
Il y a, chez les mâles, sur la région pubienne, des pores disposés sur quatre ou cinq rangées, dont deux se prolongent sur la face interne de chacune des cuisses et cessent à une certaine distance du jarret.

Les écailles qui recouvrent le museau sont plus grandes que celles des régions postérieures de la tête. Le dessus et les côtés du cou et du tronc, ainsi que la face supérieure des membres, sont protégés par des grains squamenx assez fins, serrés, égaux entre eux. Un pavé granuliforme se voit sous la tète et à la région gulaire. Des écailles à surface convexe, polygonales, un peu moins perites que celles du dos et faiblement imbriquées, couvrent la poitrine et le ventre. Il y a un indice de pli cutané le long des flancs.

Les doigts sont moins grêles que ceux des autres Gymnodactyles. Le plus externe des pattes postérieures est versatile comme dans toutes les espèces de ce genre, mais les articulations ne sont point anguleuses et les lames de la face inférieure, qui vont en diminuant insensiblement d'étendue transversale depuis la base du doigt jusqu'à son extrémité libre, sont plus larges que d'ordinaire, comme on peut le voir en comparant la fig. I 4 de la pl. xvir avec les figures 13 et I $3 a$, représentant la main et la face inférieure de l'un des doigts du Gymn. gentil. Cette différence n'est pas assez importante pour qu'on éloigne ce Geckotien du groupe auquel il apparkient par toute son organisation.

La pupille est verticale. Ce caractère, joint à la présence des pores anaux et fémoraux, et d'un faible pli cutané latéral, oblige à modifier un peu l'ensemble des caractères assignés par mon père et par Bibron au groupe des Gymnodactyles homonotes, parmi lesquels on n'en avait pas encore trouvé réunissant ces diverses particularités.

Le système de coloration offre des différences assez notables suivant l'âge, comme le montrent les diver's exemplaires des musées de Londres et de Paris.

a. La teinte générale des adultes est d'un beau vert-pré plus pâle en dessous. Sur la tête, de chaque côté, il y a une bande longitudinale arquée, d'une nuance claire, à convexité externe, puis sur la face supérieure de la queute et des membres postérieurs, des taches de la même nuance, oblongues, à contour irrégulier plus foncé. Chaque flanc, chez le mâle adulte, est orné d'une bande longitudinale blanche interrompue et bordée de noir.

b. Deux échantillons d'âge moyen sont d'un vert plus foncé, et les taches ont une teinte fauve. Sur le dos; elles ont la forme d'une losange entourés 
d'un mince filet noir, set dont lun des angles est dirigé ren avant. Sur la łète, on voit ideux lignes courbes confondues en arrière avec la premiève tache dorsale du côté correspondant. Nous avons reçu des exemplaires offramt les deux différences que je viens de signaler, par les soixrs de M. Amonx, qui les a recueillis dans la Nouvelle-Zélande.

c. Un jeune sujet enfin, semblable à un exemplaire également jeune, conservé an Mrisée britannique, est, en dessus, d'une teinte pourpre générale. relevée seulement par deux lignes courbes blanches sur les côtés de la tête Ies régions inférieures sont pâles. Ce spécimen a été donné par la 'Société zoologique. Il provient de la Terre de Van-Diemen.

A tous les âges, la lèvre inférieure est blanchâtre.

XII. - 6 bis. Granoductyle D'Arvoux, Gymnodact. Amouxii, A. 'Dum. Idem, A. Dum., Cat. des Rept. du Nus. de Paris, p. 44.

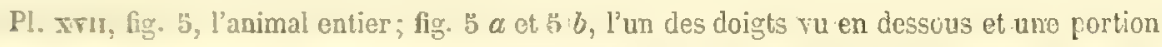
des téguments du dos.

Au miliea d'une granulation serrée, de petils tubercules arrondis, à surface convexe, sans carène, tous semblables entre eux el formant, avec une régularilé parfaite, seire rangées longitudinales; queue simplement granuleuse dans toute sa longueur et sur sa circonférence entière.

Les séries de tubercules du dos se continuent sur la tête, mais elles y. sont moins apparentes, parce que le volume de ces tubercules, qui sont d'ailleurs parfaitement semblables aux autres, est moins considérable. Elles cessent, an niveau du bord postérieur des régions sus-oculaires, ainsi que le montre la figure 5 sur laquelle ces tubercules sus-céphaliques sont d'ailleurs un peu trop volumineux. On en voit sur la face supérieure des membres postérieurs, mais il n'y en a pas sur les membres de devant.

Les régions sous-maxillaire et gulaire'sont couvertes de fines granulations qui augmentent un peu de volume à l'abdomen où elles sont rangées en séries obliques régulières, et elles ont l'apparence de petites écailles non carénées.

La queue est arrondie, conique et couverte endessous, comme en dessus, d'écailles semblables à celles de l'abdomen, mais plus grandes et non entremêlées de tubercules. Elles y forment de très-nombreux anneaux réguhiers, légèrement saillants à leur bord postérieur. 
On compte huit plaques sus-labiales à droite comme à gauche de la rostrale, qui est quadrilatère et un peu échancrée de chaque côté, à son angle supérieur et externe, par l'orifice de la narine, que bordent en haut la plaque nasale en contact avec la rostrale, en bas la première plaque de la lèvre supérieure, et en arrière, des granulations. La lèvre inférieure porte sept paires de plaques. La mentonnière est fort grande; elle a la forme d'un triangle dont le sommet arrondi, bordé de chaque côté d'une très-petite plaque ovalaire, se dirige en arrière au delà du bord des plaques labiales.

Le sujet unique du Musée de Paris manque de pores pré-anaux et fémoraux.

Ce Gymnodactyle est d'un brun plus clair en dessous qu'en dessus, où l'on voit, depuis l'occiput jusqu'à la terminaison de la queue, des bandes transversales d'un brun noirâtre, courbes, à convexité postérieure et à bords ondulés. On en compte huit également espacées depus l'occiput jusqu'à la racine de la queue. (Le dessinateur a omis la première sur la nuque.) Des taches régulières rappelant la disposition de celles du dos, occupent toute la face supérieure de la queue, dont le dessous, ainsi que le ventre et les régions gulaire et sous-maxillaire, ne porte ni taches, ni pointillé. Sur la règion occipitale, il reste quelques traces de deux bandes transversales semblables à celles du dos. Le museau est parcouru par plusieurs raies longitudinales d'un brun foncé, dont la plus apparente part du bord postérieur de l'œil pour aller se perdre sur les côtés du cou.

La forme parfaitement circulaire des tubercules cutanés, le défaut de carène à leur surface, leur très-grand nombre et l'extrême régularité de leur arrangement en séries parallèles s'opposent à toute confusion entre cette espèce et celles qui sont également hétérolépidotes. C'est surtout cet aspect globuleux des tubercules, ainsi que l'absence d'épines sur la région caudale qui, avec les différences marquées du système de coloration, éloignent le Gymnodactyle d'Arnoux des espèces également originaires de l'Australie que M. Gray a décrites (Cat. of Liz., p. 174), sous les noms de Heteronota Kendallii et $H$. Binoei.

Le Gymn. de D'Orbigny, recueilli par ce voyageur au Chili, est l'espèce la moins différente dans ce groupe, et auprès de laquelle le Gymn. d'Arnoux doit venir se placer.

Le spécimen unique de cette jolie espèce a été pris dans la Nouvelle- 
ORDRE DES SAURIENS. - GECKOTIENS.

Zélande par M. Arnoux, chirurgien de la marine à qui nous l'avons dédié. Longueur totale $\mathrm{o}^{\mathrm{m}} \mathrm{o} 85$ ainsi répartis : tête et tronc, $\mathrm{o}^{\mathrm{m}} \mathrm{0} 44$, quene, $0^{\mathrm{m}} \mathrm{o} 4 \mathrm{r}$.

Xili. - 6 ter. Grunodactyle de Perse, Gymnodact. persicus, A. Dum. ESPÈCE NOUVELLE.

Au milieu d’une granulation serrée, de nombreux tubercules irrégulièrement disposés, peu considérables, un peu coniques, surtout ceux des régions postérieures; plus volumineux sur le dos que sur les flancs et que sur la téte, où ils ne s'étendent pas au-devant des yeux; tête courte et épaisse; queue cylindrique, de longueur médiocre, verticillée et fort grêle, ainsi que les membres.

La conformation générale est assez remarquable, en raison de la gracilité des membres et de la queue relativement au tronc, qui est plutôt trapu. La tête a peu de longueur, elle est épaisse, bombée an niveau des orbites, d'où résulte une déclivité très-prononcée du museau, qui est court et arrondi.

Les granulations des régions supérieures sont irrégulièrement polygonales, et les petits intervalles qu'elles laissent entre elles sont remplis par d'autres granulations d'un volume beaucoup moindre, triangulaires et disposées de façon à former une sorte de couronne autour de chacun des tubercules, qui sont en grand nombre, et non groupés en séries régulières. Sur la tête, ils sont plus petits que partout ailleurs, plats ou un peu globuleux; sur le tronc, au contraire, leur partie moyenne est légèrement proéminente, et ceux des régions postérieures sont coniques. Il y en a sur les membres et sur la queue, où ils forment, dans chaque anneau, le rang antérieur, celui qui par la saillie qu'il présente rend apparente la disposition verticillée des écailles caudales. A la base de la queue, de chaque còté, on voit deux ou trois tubercules rapprochés et saillants.

Le ventre est revêtu d'écailles polygonales et plus grandes que les granulations de la gorge, qui sont très-régulières et toutes de même dimension, excepté celles bien plus volumineuses des deux ou trois rangs situés immédiatement derrière les plaques sous-labiales. Ces dernières sont au nombre de 21 à 25 , en y comprenant la mentonnière, qui offre des dimensions médiocres. On en compte 24 à la lèvre supérieure séparées par la rostrale; celle-ci est double, et chacune de ses moitiés est à peu près semblable à la plaque dont elle est suivie, si ce n'est qu'elle est un peu plus haute. Cette rostrale concourt, de chaque côté, avec la première plaque de la lèvre supérieure et avec trois petites plaques nasales postérieures à 
circonscrire la narine, dont l'orifice est presque terminal. Les denx ouvertures nasales sont petites et très-rapprochées l'une de l'autre.

La pupille est verticale. Il n'y a point de pores pré-anaux ni fémoraux.

La teinte générale, autant qu'on peut en juger, malgré la destruction presque complète de l'épiderme, paraît être, en dessus, un brun peu foncé. Depuis la nuque jusqu'à l'origine de la queue, il y a cinq larges bandes trans versales plus sombres. On en voit de semblables, mais plus étroites, et qui sont par conséquent plus nombreuses sur la queue et sur les membres, et jusque sur les doigts. La gorge est vermiculée de petits traits bruns. Tout le reste des régions inférieures est d'un brun-jamne clair et uniforme. Il n'y a point de taches ni de lignes sur la tête.

L'énumératioñ de tous les caractères qui précèdent ne laisse aucun doute sur leur valeur comme marques distinctives propres à éloigner cette espèce jusqu'ici inédite de toutes celles que comprend le genre auquel elle appartient.

Elle diffère, en effet, par sa conformation générale, la gracilité des membres et de la queue, par la forme de la tête, par l'aspect même des tubercules et leur irrégularité, des espèces dites Gymn. de D'Orbigny, d'Arnoux et à bandes, qui sont celles dont elle s'éloigne le moins.

La description qu'on vient de lire est faite d'après trois individus parfaitement semblables entre eux et rapportés par Aucher-Eloy de són voyage dans le Levant, et particulièrement dans la Perse : d'où le nom spécifique dont j'ai fait choix pour ces nouveaux Gymnodactyles. - Le plus grand est long de $\mathrm{o}^{\mathrm{m}}$ I I ainsi répartis : téte et tronc, $\mathrm{o}^{\mathrm{m}} \mathrm{o} 6$, queue, $\mathrm{o}^{\mathrm{m}} \mathrm{o} 5$.

XIV. - 8 bis. Gymnodactyle caspien, Gymnodactylus caspius, Eichwald.

Idem, Eichw., Zool. specialis Rossiæ et Polon., pars posterior, p. 181, et Fauna caspio-caucasia, 1841, p. 91, tab. xv, fig. 1-2. - Uromastix fasciatus, Ménestriés, Catal. raisonné, n० 220. G.ymn. caspius, Eichw., Catal. des Rept. du Mus. de Paris, p. ¿́. -Id., Id., Répert. Erpét. génér., Dum. Bib., t. IX, p. $25 \dot{x}$.

Sur le dos, des écailles granuleuses entremélées de tubercules beaucoup plus grands, scillants, fortement carënés, ayañt la forme de petites pyramides triangulaires et disposés en séries longîludinales assez régulières sur le dos et en verticilles sur la queue; des tubercules sur les membres; tete large; museau mousse et arrondi; des pores pré-anaux et fémoraux.

La description très-complète donnée par M. Eichwald, et la figure jointe à 
son texte, dispensent d'indications détaillées sur ce Gymnodactyle, dont je panle ici parce que le Musée de Paris en possède un très-bel exemplaire adressé de Saint-Pétersbourg par M. Ménestriés depuis la publication du t. III de l'Erpét. génér. Je tiens d'ailleurs à relever les différences remarquables qui le séparent du Geckotien que M. Rüppell a le premier fait connaitre sous les noms de Stenodactylus scaber, et avec lequel différents zoologistes, et en particulier MM. Fitzinger (Syst., p. $9^{3}$ ) et Gray (Catal., p. 175), le confondent.

Si donc, nous les comparons l'un à l'autre, nous voyons chez le Gymnod. caspien les particularités suivantes.

$1^{\circ}$ I a granulation du dos entre les tubercules est beaucoup moins fine et moins régulière; ces tubercules sont plus volumineux et plus espacés, et en outre, sur la ligne médiane du dos, il y en a de même forme, mais plus petits, disposés en rangée longitudinale. - $2^{\circ}$ Les squames du ventre sont moins grandes et groupées de façon à former des lignes plus obliques. $3^{\circ}$ La tète est plus large et le museau plus arrondi et plus mousse. $-4^{\circ} \mathrm{La}$ plaque mentonnière représente un triangle plus allongé, à sommet plus pointu, d'où résulte un écartenent plus considérable des deux plaques entre lesquelles ce sommet pénètre. - $5^{\circ}$ Les formes sont plus robustes; les membres sont moins longs et moins grêles. $-6^{\circ}$ Les pores, au lieu de n'occuper que la région pré-anale, s'étendent en outre sur chaque cuisse et forment ainsi une ligne courbe continue. - $\eta^{\circ}$ Enfin, les régions supérieures portent des bandes en travers et non des lignes longitudinales.

XV. - 8 ter. Granodactyle coléonyx, Gymnodactylus coleonyx. Dum.

Coieonyx elegans Gray (Annals and magaz. of nat. hist. Sept. 1845, t. XVI, p. 462). - Gymnodact. scapularis, A. Dum., Catal. des Rept. du Mus. de Paris, p. 4... - Id., Id., Répert. Erpét. gẻn., Dum. Bib.; t. IX, p. 2ä̌.

Pl. xv7r, fig. 6 , l'animal entier, $6 a$, sa main vue en dessous; $6 b$, l'un des doigts; $6 c$, région anale.

Doigts à peu près cylindriques, mousses à leur extrémité, qui est munie de deux longues écailles latérales, un peu étraites, légèrement convexes, formant pour l'ongle qui est court une gaine où il peut se cacher entierement, et complétée en dessus par une écaille moins longue.

Cette conformation particulière des doigts suffit pour éloigner ce Gymno- 
dactyle de tous ceux que l'on connait. C'est ce que j'avais indiqué dans notre Cútalogue, où je l'ai désigné par les noms de Gymn. à scapulaire, en faisant observer qu'il pourrait être considéré comme le type d'un sous-genre. Ces noms ne doivent plus être conservés maintenant. Ceux de Coléonyx élégant que lui a donnés M. Gray, quelques mois après la publication de son Catal. où cet animal n'est pas mentionné, seraient admis dans ce Mémoire s'il n'y avait nécessité de laisser à ce Gecko la dénomination générique de Gymnodactyle rappelant la grande division à laquelle il appartient. Nous le nommons donc au Musée de Paris Gymn. coléonyx, ou Coléonyx élégant, si on le considère comme le type d'une subdivision dans le genre Gymnodactyle.

J'ai d'ailleurs constaté l'identité de notre espèce et de celle du Musée de Londres. Elle a été confirmée par M. Gray lui-même, car il a pu comparer la fig. 6, de la pl, xvi avec l'individu qui a servi à sa description, et dont la patrie est l'Amérique centrale, comme pour le nôtre. Celui-ci est sans doute plus âgé. C'est du moins ce que semble indiquer l'absence, sur la tête, des lignes concentriques noires visibles chez le sujet du Musée britanique et remplacées ici par une large bande blanche bordée de noir, qui dessine le contour de la région occipitale en s'étendant jusqu'aux yeux.

J'ajouterai maintenant quelques détails pour compléter la description.

La tête est volumineuse, longue et large en arrière. Le museau est conique. La plaque rostrale est triangulaire, et son sommet se prolonge un peu en arrière; à droite comme à gauche, elle touche, par sa portion supérieure, aux deux plaques nasales qui circonscrivent l'orifice de la narine. On compte sur chaque moitié de la lèvre supérieure six plaques, dont la deuxième a beaucoup plus de longueur que tontes les autres. Le tronc est gros et les membres sont médiocrement robustes. La queue manque presque complétement sur notre exemplaire, mais nous voyons dans la description de M. Gray qu'elle est cylindrique et porte des anneaux formés par des tubercules sub-anguleux, dont le volume dépasse celui des écailles situées entre les verticilles. Elle est renflée en dessous près du cloaque et armée de chaque côté, à sa base, de gros tubercules.

L'écaillure du tronc se compose de granulations fines et régulières, entremêlées de nombreux tubercules un peu coniques, disposés en séries, ils sont plus petits et plus espacés sur la nuque et sur la tête que sur le dos; sur les reins, au contraire, leur nombre augmente. Ils sont assez abondants sur les 
membres postérieurs, tandis que l'écaillure des antérieurs est homogène et uniquement composée de petites squames un peu imbriquées.

Les régions sous-maxillaire et gulaire sont couvertes de grains très-fins, au milieu desquels il s'en trouve quelques-uns plus volumineux, rassemblés de manière à former un petit groupe de forme irrégulière placé sur la ligne médiane, et ne dépassant pas en arrière le niveau de l'angle de la mâchoire inférieure. L'abdomen est revêtu d'écailles polygonales, un peu imbriquées, plus grandes et assez manifestement triangulaires à la région pré-anale, qui porte une série angulaire de pores.

Sur un fond brun-grisâtre, trois larges bandes d'un brun foncé occupent en dessus presque toute la longueur du corps, car elles ne laissent entre elles que des intervalles étroits couverts par la teinte du fond. Sur le cou, il y a une tache semblable aux bandes : elle se termine, en arrière, par un prolongement sur chacune des épaules, et en envoie à la région antérieure deux autres qui s'étendent sur les còtés de la tête, dont le dessus est orné d'une bande noirâtre en fer à cheval. Entre cette bande et les deux prolongements antérieurs de la tache du cou que je viens de décrire, la teinte grise du fond apparaît sous forme d'une bande de même forme que celle en fer à cheval et de nuance brune qui lui est concentrique. - J'ai déjà signalé la différence que présente, sous ce rapport, le spécimen du Musée de Londres, chez lequel cette bande claire à bords foncés est remplacée par plusieurs lignes noires, ce qui, suivant M. Gray, indiquerait que l'animal qui lui a servi de type est plus jeune que le nôtre. - A la naissance de la queue, dont il ne reste qu'un tronçon, il y a une tache transversale semblable à celle du dos. - Les parties inférieures sont d'un gris jaunâtre uniforme.

La longueur, depuis l'extrémité du museau jusqu'à la racine de la queue, est de $0^{\mathrm{m}} \circ 9$, ainsi répartis : tête et cou, $0^{\mathrm{m}} 04$, tronc, $\mathrm{o}^{\mathrm{m}} \mathrm{0} 5$; queue mutilée.

C'est de M. Arthur Morelet que le Muséum a reçu ce curieux Gecko. Il l'a recueilli dans la province du Peten, pendant l'important voyage qu'il a accompli à travers les contrées si peu explorées de l'Amérique centrale, d'où provient également l'individu décrit par M. Gray ${ }^{1}$.

1. Aux Gymnodactyles nouveaux inconnus à Paris, il faut ajouter les suivants, décrits par M. Jerdon Catal. of Rept. inhabiting the peninsula of India (Journ. of the asiat. soc. of Bengal, 1853, 1. XXII, p. 469 et suiv.): G. malabaricus, littoralis, mysoriensis. - Nous ne connaissons pas non plus d'autres Geckotiens signalés par le mème zoologiste dans ce Journ., p. 467 et 468. Il les nomme 


\section{GENTE STENOTACTYUE. STENOTACTYLES. FITZ.}

Pl. xvir, fig. 15,16 et $16 a$.

Les détails dans lesquels je suis entré en commençant la révision du genre Gymnodactyle ont montré le lien qui, dans les méthodes de différents zoologistes, réunit tous les Geckos à doigts non dilatés, c'est-à-dire ceux que les auteurs de l'Erpét. génér. ont décrits sous les noms de Gymnodactyles et de Sténodactyles, et qui, par conséquent, diffèrent beaucoup, par leur genre de vie, des autres Geckotiens.

Le genre Sténodactyle, tel qu'on peut l'admettre dans les limites étroites que M. Fitzinger d'abord, puis mon père et Bibron lui ont assignées, est trèsbien caractérisé par les granulations de la face inférieure des doigts et par les dentelures de leurs bords indiquant des animaux fouisseurs ${ }^{1}$. Il s'éloigne d'une façon notable des Gymnodactyles, dont tous les doigts, sans dentelures latérales, sont munis en dessous d'écailles transversales. En outre, le cinquième, chez les Sténodactyles, n'est pas versatile, ce qui s'oppose à ce que, comme les précédents, ils puissent grimper sur les arbres.

M. Fitzinger, dans son Syst., place à la suite du genre Sténodactyle, le genre Eublepharis, Gr., dont le type Eubl. Hardwickï, Gr. est inconnu au Musée de Paris, et qui paraît se rapprocher plus des Gymnodactyles que des espèces comprises dans le genre dont nous avons maintenant à nous occuper. Les doigts, en effet, chez l'Eublepharis, ne sont ni granuleux en dessous, ni dentelés sur leurs bords; leur face inférieure, au contraire, est protégée par des lamelles transversales. Aussi M. Gray place-t-il ce genre à la suite des Naultinus et avant son genre Homonota, qui a pour type le Gymnodact. de Gaudichaud, Dum. Bib.

Outre le Stén. tacheté, on en connaît deux auires très-distincts: l'un de

Hemidact. sub-triedrus, n. spec.? (H. triedrus, var.?), Hem punctatus, Homonota fasciata. Dans ce mème vol., p. 646, M. Blyth mentionne un autre Hémidact. indien: Leiurus Berdmorei. -Enfin, parmi les nouveaux Geckos, il y a (Proc. Philad., mars 1852) Hemidact. angulatus Hallow. de la côte occident. d'Afr. et Pachydact. tristis Hallow. (même Recueil, juin 1854) de Liberia.

1. Dans un Mém. (Revue de zool., 1851, p. 479) oủ j'ai décrit le Sténodact. queue-cerclée, dont il est question plus loin, j'ai cherché à montrer par la plupart des exemples connus, la corrélation remarquable qui existe non-seulement chez les Sauriens, mais chez certains insectes, entre la présence de dentelures sur le bord des doigts et le genre de vie, les animaux ainsi conformés étant tous plus ou moins fouisseurs. 
l'Afrique du Sud, inscrit par M. Smith sous les noms de St. garrulus, et l'autre, du Sénégal, que j’ai nommé St. caudicinctus.

Un troisième ( $S t$. mauritanicus) recueilli en Algérie, décrit et figuré par M. Guichenot, dans l'Erpétologie de cette contrée, ressemble beaucoup à l'espèce type (St. elegans, Fitz., ou St.guttatus, Cuv.).

M. Eichwald (Naturhist. Bemerkungen über Algier, und den Atlas in Nouץ. Mém. de la Soc. impér. des natural. de Moscou, t. IX, I85\&, p. 419) dit en parlant du St. tacheté, qui est compris parmi les animaux qu'il décrit comme vivant en Algérie, que probablement il y a identité entre ce Geckotien et le St. mauritanique. La distinction est, en effet, fort difficile à établir, tant les analogies sont frappantes. La comparaison, d'ailleurs, ne peut pas être complète sous tous les rapports, avec le type égyptien, qui est unique au Musée de Paris, et dont les couleurs sont fort altérées.

XVI. - I bis. Sténodactrle mauritanioue, Stenod. mauritanicus, Guich.

Idem, Guich., Explor. scientif. de l'Algérie, Rept., p. b, pl. I, fig. 1 et fig. $a, b, c, d$.

Les différences caractéristiques se remarquent particulièrement, comme le fait observer le zoologiste qui a, le premier, décrit cette espèce, dans la disposition des couleurs. Voici les détails qu'il a donnés à ce sujet :

« Le dessus de la tète, dit-il, est d'un gris ardoisé marqué de points pâles et de traits diversement disposés sur le crâne; le corps et la queue présentent un gris foncé qui passe au vert fauve sur la région dorsale, avec de trèslarges bandes brunes transversales et des gouttelettes jaunes apparaissant sur la ligne médiane de la queue sous la forme de grandes taches rondes et séparées de cette dernière couleur. Une teinte d'un gris fauve règne sur les régions inférieures, et les membres, dans toute leur étendue, sont semés, sur un fond grisâtre, de taches ou traits d'un bleu pur."

Le Musée possède les trois individus qui ont servi pour cette description. Ils ont été prís à Oran par M. Guichenot et par MM. Levaillant et Bravais.

Un nouvel examen d'un spécimen envoyé d'Afrique par M. Botta, nous montre son analogie parfaite avec le St. tacheté, dont nous rapprochons également deux exemplaires récemment reçus d'Algérie, par les soins de $\mathbf{M}$. le général Daumas. - Il n'y a pas de différence notable entre cette espèce et un sujet qui porte pour renseignement qu'il a été recueilli en Australie, sur 
les bords de la Baie des chiens marins. Cependant, en raison de l'identité spécifique incontestable de ce Geckotien et du St.tacheté, je crains que cette indication ne soit inexacte.

XVII. - I ter. Sténodactyle babiliard, Stenodactylus garrulus, Smith.

Idem, Smith, Illustr. of the zool. of South Afr. Appendix, p. 6. - Id., Catal. des Rept. dut Musée de Paris, p. 47. - Id., Répert., Erpét. génér., Dum., Bib., t. IX, p. 255.

Têle fort courte, large, bombée, à museau obtus et très-déclive; ouverture de la bouche peu considérable; sept plaques à la lèvre supérieure de chaque cóté de la rostrale et sept à la lèvie inférieure de chaque cólé de la mentonnière; membres trapus et robustes; queue courte, conique et assez volumineuse.

Cet ensemble de caractères permet très-facilement de distinguer cette espèce du Sténodactyle tacheté, qui a la tête plus longue, le museau moins court, moins obtus, beaucoup moins déclive, l'ouverture de la bouche plus considérable et qui, par suite, porte un plus grand nombre de plaques labiales, car on en compte douze de chaque côté de la rostrale, comme de chaque côté de la mentonnière. Enfin, les membres sont proportionnellement un peu plus longs et plus déliés que chez le Stén. babillard, puis la queue est plus grêle et légèrement plus allongée.

On peut ajouter, pour compléter la description de cette espèce nouvelle, que les régions supérieures sont couvertes de petites granulations plus fines encore sur la tête que sur le dos; il en est de même pour celles de la gorge, qui sont inférieures en volume à celles du ventre, où déjà elles ont un diamètre très-peu considérable. La queue est simplement granuleuse dans toute son étendue, en dessus et en dessous. Sur les côtés du cou, la peau est lâche et se gonfle à la volonté de l'animal.

Les couleurs de notre spécimen sont un peu altérées par l'action de l'alcool; on y retrouve pourtant des traces du système de coloration que M. Smith décrit ainsi :

Les parties supérieures et latérales, le dos, les flancs et la queue sont d'une couleur orange pâle; près du cou, le dos est varié de lignes transversales étroites et ondulées, d'un rouge brunâtre ; le reste du dos est bigarré de petites taches irrégulières de la même nuance. La queue est tachetée de rougebrun; les régions inférieures sont d'un blanc jaunâtre. 
I. Smith donne les détails suivants sur les mours bizarres de cetle jolie petite espèce : « Le Sténodactyle babillard, dit-il, habite les contrées sablonneuses de l'Afrique australe. Il vit en troupes et habite de petits terriers presque perpendiculaires. Il cherche probablement sa nourriture pendant la nuit; du moins, pendant le jour, je n'ai jamais vu que sa tête au-dessus du sol; on peut alors, dans les localités où on le rencontre, en voir un grand nombre d'individus qui regardent, réfugiés dans leur cachette, en produisant chacun un cri aigu, comme chik-chik; et la multitude d'animaux se livrant ensemble à cette occupation est telle, et le bruit qui en résulte est si désagréable, que le voyageur est forcé de changer ses quartiers. ”

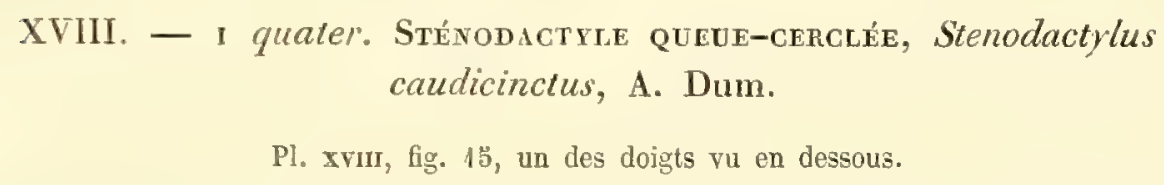

Idem, A. Dum., Cat. des Rept. du Mus. de Paris, p. 48. - Id., Revue de zool., Oct. 1854, no 10, p. 479, pl. xu1. - Id., Répert., Erpét. génér., Dum., Bib., t. IX, p. 255.

Tubercules nombreux, semés avec régularité au milieu de la granulation générale des parties supérieures; queue robuste, entourée, dans toute sa longueur, de larges anneaux très-réguliers, qui sont armés en dessus de tubercules volumineux.

Ces caractères suffisent pour établir une distinction tranchée entre ce Sténodactyle et ses congénères, car il est le seul dont l'écaillure ne soit pas homogène et qui, par les verticilles tuberculeux de la queue, ait une certaine analogie avec les Iguaniens nommés, en raison de cette particularité, Oplures, tromastyx, etc.

Les formes sont lourdes. La tète est volumineuse, large en arrière, assez allongée, à museau conique. La queue renflée à la partie moyenne s’amincit rapidement vers sa pointe. Outre sa grosseur qui est assez considérable, elle est longue, car elle n'a que $0^{\text {m }}$ or o de moins que le tronc. - Les membres sont robustes et les doigts granuleux en dessous et dentelés sur leurs bords, comme chez tous les Sténodactyles. Les cuisses et les jambes portent en dessus des tubercules qui manquent aux membres antérieurs.

Il y a, sur la région pré-anale, une rangée de pores.

Sur les parties latérales du dos et du cou, les tubercules sont réunis trois à trois : un volumineux au milieu, bordé, de chaque côté, par un autre tuber- 
cule plus petit qui lui est adhérent. Sur la région médiane, ils sont isolés. A la face supérieure de la tête, ils sont plus serrés que partout ailleurs, et sur les verticilles de la queue, plus saillants que dans aucun antre point.

La gorge est recouverte de petites squames juxtaposées régulières, presque circulaires. On en voit cependant quelques-unes plus grandes en avant; il y en a deux, en effet, qui bordent la mentonnière et qui sont elles-mêmes suivies de deux autres d'une dimension un peu moindre. En dehors de chacune des plaques antérieures de ce petit groupe formé par les quatre que je viens de signaler, on en trouve, le long du bord inférieur de la mấchoire, une rangée bientòt confondue avec les autres pièces du pavé granuliforme environnant, mais dont les cinq ou six premières, de moins en moins grandes, l'emportent toutes cependant par leur volume sur celles qui les entourent. Les écailles du ventre et de la région sous-caudale ont des dimensions plus considérables que celles de la gorge; elles sont faiblement imbriquées et arrondies à leur bord postérieur. - De chaque côté de la plaque rostrale, qui est dilatée en travers, et dont le bord supérieur est un peu ondulé, il y a douze labiales.

La teinte générale est un brun grisâtre tirant sur la couleur lilas, orné en dessus de trois grandes taches d'un brun violacé. La première, en forme de fer à cheval, commence, de chaque côté, derrière l'oeil et s'arrondit sur l'occiput. La deuxième, à peu près quadrilatère, couvre les épaules. La troisième a la forme d'un large triangle, dont la base concave se termine en avant par deux prologements pointus, qui s'avancent sur les flancs, et son sommet trèsaigu s'arrête sur la ligne médiane, au niveau de la naissance des membres postérieurs. Sur la queue, il y a quatre demi-anneaux de la même nuance que les taches, et le dernier se confond en partie avec le troisième. En dessous, la couleur est partout un brun grisâtre uniforme d'une teinte plus claire que celle des régions supérieures.

Outre ces divers caractères, qui établissent des différences si tranchées avec les autres espèces du même genre, il faut encore citer la grande taille de ce Gecko, car il les dépasse tontes. Sa longueur totale est de $0^{\mathrm{m}} 155$ ainsi répartis : tête, $\mathrm{o}^{\mathrm{m}} \mathrm{O}_{2} 5$, tronc, $\mathrm{o}^{\mathrm{m}} \mathrm{o}$ \% $\mathrm{o}$, queue, $\mathrm{o}^{\mathrm{m}} 060$.

Le Muséum ne possède qu'un seul spécimen type de cette espèce nouvelle. II est originaire du Sénégal, et dans un très-bon état de conservation. 


\section{QUATRIEME: FAMLLE : VARANIENS OU PLATYNOTES.}

Les Reptiles réunis sous le nom de Varans forment une famille fort naturelle, qu'il est aussi facile d'isoler du reste des Sauriens que les trois familles précédentes. Elle comprend, en effet, des Reptiles de taille assez grande, trèsnettement caractérisés par leur écaillure composée de granulations tuberculeuses semblables sur tonte la surface du corps, et par la conformation de leur langue charnue, longue, protractile, profondément bifide et engainée dans un fourreau. C'est cette dernière particularité que Wagler a voulu désigner, quand il a fait usage de la dénomination de Thecoglossae (langue dans une gaîne).- Mon père a exposé dans le t. III de l'Erpét. génér., l'historique de la classification de cette famille. Je dais me borner à rappeler ici les nouveaux travaux sur ce sujet. - M. Fitzinger (Syst. 1843), a modifié son classement de 1826 . Partageant tous les Reptiles en 5 grandes séries: Amblygloss压, Fitz., Leptoglossæ, Fitz.(Wiegm.), Testudinata, Oppel, Drpmos, Leuckart, et Rhizononta, Fitz., c'est dans la deuxième que sont introduits les Thécoglosses, qui constituent une première tribu dans la section (dite des Pléodontes), placée elle-même en tête de l'ordre des Sauriens.

Cette tribu des Thécoglosses est formée par la réunion de quatre familles. La première ( Palceosauri) ne comprend que des genres fossiles; dans la quatrième, on trouve les Améivas et quelques groupes voisins réunis sous le nom de Podinema. C'est à la troisième (Polydadali) que les vrais Varans sont rapportés. La deuxième enfin (Helodermata) ne se compose que d'un seul genre, celui que Wiegmann a établi sous le nom de Heloderma.

Avant d'étudier les Varans proprement dits, j'ai à mentionner les recherches récentes sur l'Héloderme, entreprises par M. Troschel qui, en faisant connaitre avec un assez grand nombre de détails la structure de ce Reptile presque inconnu des naturalistes jusqu'à ces derniers temps, a jeté un nouveau jour sur son histoire, et a montré ses véritables affinités zoologiques (Ueber Heloderma horridum, Troschel's Archiv. fur naturgeschichte, I853, I 9 année, t. I, p. 294, pl. xirr und xiv).

Ce Saurien, que le Musée de Berlin a longtemps possédé seul, et qui fut primitivement décrit et figuré par Wiegmann, n'avait jamais été mentionné depuis, que d'après le dessin et le texte du célèbre naturaliste prussien. Le Musée Britannique cependant en possède aujourd'hui deux individus, l'un 
adulte et l'autre plus jeune. La langue malheureusement n'a pu être étudiée, et M. Gray a dû forcément se borner à reproduire les indications fournies par l'Erpét. du Mexique, en considérant ce lézard, le seul que renferme le genre unique Héloderme, comme type, dans son Cat. of Liz, de la famille des Hélodermides, qu'il place à la suite de celle des Monitorides (Varans) laquelle, dans son Système, est en tête de la tribu des Cyclosaures, qu'il considère comme devant être la première du sous-ordre des Leptoglosses.

L'animal que M. Troschel a examiné est en assez mauvais état sous certains rapports seulement, et il est conservé dans l'alcool au riche Musée de Bonn. Ce zoologiste a constaté son identité spécifique avec l'Héloderme hérissé, Wiegm.

Beaucoup de circonstances importantes à noter relativement au squelette, sont signalées dans son Mémoire, mais je dois m'en tenir dans cette analyse aux faits les plus saillants.

Celui qu'il faut signaler tout d'abord est relatif à la conformation de la langue qui, un peu bifide en avant, il est vrai, mais beaucoup moins que ne l'est celle des Varans, ne peut pas, comme chez ceux-ci, rentrer dans une gaîne ou fourreau membraneux. Elle n'est longue que de $o^{\mathrm{m}} \mathrm{o} 38$ (la tête mesurant $\mathrm{o}^{\mathrm{m}} \mathrm{ogo}$ ), et chacune de ses pointes y est comprise pour $\mathrm{o}^{\mathrm{m}}$ or o.

Sa surface, loin d'être molle et charnue, est recouverte de papilles écailleuses assez grandes à la base de l'organe, mais qui diminuent insensiblement en avant, de sorte que les deux pointes sont presque lisses.

Voici d'ailleurs les conclusions que M. Troschel tire de l'étude des parties dont il a pu faire un examen suffisamment approfondi.

"Si maintenant, dit-il, nous nous demandons quelle est la véritable place de l'Héloderme, c'est surtout la forme de la langue qui nous guidera pour notre réponse, car cet organe, dans tous les systèmes récents de classification des Sauriens, est considéré comme l'un des plus importants. Fort heureusement, il a été conservé dans notre exemplaire, tandis qu'il n'a pu être étudié par M. Wiegmann qui, d'après la description de Hernandez, où la langue est désignée comme protractile, large et bifide, a rangé ce Lézard parmi ses fis silingues, et c'est seulement cette description qui le porte à conclure qu'elle peut se retirer dans une gaîne ${ }^{1}$. Nous avons dit qu'il n'y a pas trace de ce

1. Je ferai observer qu'on peut, avec M. C. Ranzani (de Tupinambididus, 1836, p. 21), s'étonner 
fourreau. La langue, en outre, dans sa conformation générale, n'est pas semblable à celle des Varans, tandis qu'elle présente une grande ressemblance avec celle des brevilingues de Wiegmann, et surtout avec celle des Reptiles compris dans la division de ce groupe où il décrit cet organe comme oblong, bifide et écailleux, division dont il forme une famille spéciale comprenant les Lézards proprement dits dans l'ordre des Sauriens. "

“Les apparences extérieures ne s'opposent pas à ce que l'Héloderme soit placé dans la famille des Lacertiens, Dum. Bib., et même la forme quadrilatérale des écailles du ventre est un caractère qui confirme cette classification. »

"Un seul doute peut maintenant s'élever. Ce genre doit-il prendre place dans la famille même des Lacertiens, ou ne pourrait-il pas devenir le type d'une famille voisine? Le squelette differe certainement, sous bien des rapports, de celui des Lézards propres, mais comme il en est probablement de même pour plusieurs autres genres considérés cependant comme peu éloignés les uns des autres, je n'hésite pas à ranger l'Héloderme dans cette famille des Lacertiens, car il présente la plupart des caractères que MM. Duméril et Bibron ont employés pour en tracer la délimitation dans l'ordre des Sauriens. „ (Traduction inédite de M. Lobligeois.)

Je reviens aux Varans proprement dits. Je dois d'abord rappeler un savant travail de M. Ranzani, publié en 1836 , dans la même année que le t. III de l'Erpét. génér., où il n'a pas pu être cité. Cet habile zoologiste y a très-bien indiqué leurs véritables affinités, après avoir discuté les modifications successives que leur classification avait subies jusqu'à cette époque.

Quant aux divisions et subdivisions ultérieures proposées par MM. Fitzinger et Gray, ainsi que pour les derniers travaux de M. Schlegel sur ce sujet, je me bornerai à en présenter un court résumé.

Voici le prodrome de M. Fitzinger (Syst. Rept., p. 19), et dans lequel le mot Varanus, employé d'abord par Merrem, ne se retrouve plus:

Fam. 3. POLYDEDALI

1 Gen. Hronosacbes Wagl. : 2 sub-gen. a Cylindrurus Filz. (Australia) : Odalria punctala Gras; $b$ Hydrosaurus Wagl. Asia, Aastr.: Hydros. bivillatus Wagl.

2 Gen. Eupreplosanums Fitz. : 3 sub-gen a Pantherosauru Pilz. (Aa:tr.) : Hydros. Gouldii Gr; b Agulmalosurus Filz. Lsia): Varanus Tworiensis Dam. Bib.; c Euprepiosanrus Fitz. (Austr.) : Varanus chlorostigma Dam. Bib.

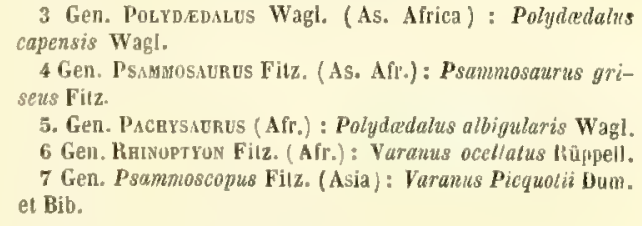
capensis Wagl.

4 Gen. Psammosiurus Fitz. (As. Afl') : Psammosaurus griseus Fitz.

5. Gen. Paceysarrus (Afr.) : Polydadalus albigularis Wagl. 6 Gen. Rhisoptyon Filz. (Afr.): Varanus ocellatus liủppell. 7 Gen. Psammoscopus Filz. (Asia): Varanks Piequotiz Dum. et Bib.

que Wiegmann et Wagler, sur une si vague indication qu'il leur avait été impossihle de vérifier, ajent fait de ce lieptile un Thécoglosse. Aug. D. 
On doit beaucoup regretter que le savant zoologiste de Vienne n'ait pas encore fait pour toute la classe des Reptiles un travail semblable à celui qu'il a déjà publié sur la première série, celle des Amblyglosses. On y trouverait l'énumération des caractères sur lesquels il s'est appuyé pour diviser le groupe des Varaniens en 7 genres et en 5 sous-genres, et je n'aurais pas à m'en tenir à ce simple énoncé malheureusement insuffisant pour permettre d'apprécier la valeur de cet arrangement systématique.

M. Gray (Cat. of Liz., p. 6) partage la famille (Monıtoride) en deux groupes:

I. M. à queue ronde, sans crête en dessus (terrestres) : 2 genres.

II. M. ̀̀ queue surmontée d'une carène comprimée formée par deux rangs d'écailles (aquatiques): 5 genres.

On compte dans ces différents genres vingt-trois espèces, onze de plus que les douze qui étaient connues à l'époque de la publication de l'Erpét. génér. J'en présente ici la liste en désignant par des caractères italiques celles qui ne sont pas mentionnées dans ce dernier ouvrage.

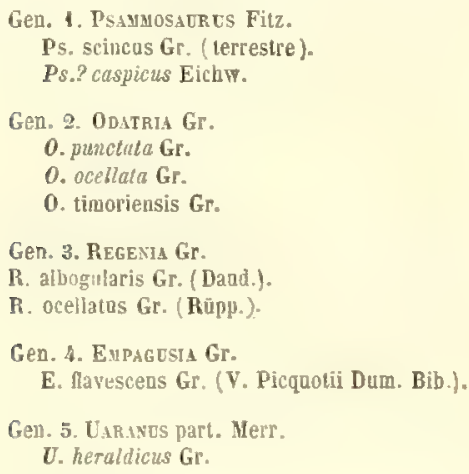

V. lunatus Gr.

U. ornatus Gr.

U. Dumerilii Nüll.

U. rudicollis $\mathrm{Gr}$.

C. nebulosus Gr. (Cav.).

Gen. 6. Moxiton ( Cav.) Gr.

M. niloticas Gr. (Hasselq.).

M. dracæua Gr. (Linn.), (Tup. bengaleusis Daud.).

M. Gouldii Schl.

M. chlorostigma Cay

Gell. 7. Hyorosagnes Wagl

H. varius $\mathrm{Gr}$.

H. Bellii Dum. Bib.

H. giganteus $\mathrm{Gr}$.

H. salvator, Gr. (Tup, bivittatus Kuhl.).

H. prosinnus Mừlı.

Les divisions et subdivisions êtablies dans un genre si homogène et si parfaitement naturel que l'est celui des Varans, sont toutes plus ou moins systématiques et artificielles. Elles tendent, sans motifs suffisants, à faire considérer comme appartenant à divers genres des animaux qui n'offrent réellement entre eux que des différences spécifiques. Aussi M. Schlegel qui, dans son Essai sur la physionomie des Serpents, a manifesté un éloignement extrème pour l'adoption des coupes plus ou moins nombreuses proposées par ses devanciers, s'est-il montré fidèle à ses habitudes comme zoologiste, en n'admettant qu'un seul genre, celui des Monitors, dans l'explication mé- 
thodique et savante qu'il a donnée des figures publiées par lui (Abbildungen neuer oder unvollständig bekannter amphibien, 1837-44).

En laissant de côté la différence de dénomin ation générique sur laquelle il est inutile d'insister, on voit qu'il y a conform ité de vues entre ce naturaliste et les anteurs de l'Erpét. génér. touchant l'analogie extrême qui se remarque entre les diverses espèces.

Il y a néanmoins divergence relativement à la division établie dans ce dernier ouvrage d'après la forme de la queue, et qui consiste à ne considérer comme essentiellement terrestres, que les $V$. du désert et de Timor, chez lesquels elle est cylindrique, et comme aquatiques tous ceux où elle est comprimée.

"C'est par erreur, dit M. Schlegel, qu'on regarde ces derniers comme appelés à vivre dans les eaux et qu'on tient pour terrestres les Varans à queue ronde. Le fait, vrai en général, souffre ici des exceptions. C'est ainsi que le Monitor exanthematicus ( $V$.ocellatus, albigular is et Picquotii, voir plus loin l'analyse méthodique du groupe tel qu'il l'a établi) ne va jamais à l'eau, quoiqu'il ait la queue comprimée; d'un autre còté, elle est tout à fait ronde chez le Monilor Timoriensis, qui a non-seulement la conformation, mais jusqu'à la couleur des Varans aquatiques. ”

Celui-ci, cependant, tant qu'on n'aura pas la preuve positive du contraire, semble devoir être rapproché du $V$. arenarius, dont il doit avoir les mours et les habitudes.

"Quant au Monitor prasinus, ajoute M. Schlegel, sa queue plus haute que large et sans crête, ses formes sveltes, ainsi que sa belle couleur verte, indiquent bien qu'il fait surtout des bois son habitation ordinaire."

Je crois devoir présenter ici les faits suivants rapportés par le même naturaliste, car ils compléteront les détails que les auteurs de l'Erpét. gérér. ont donnés sur les møurs intéressantes de ces grands Sauriens qui peuvent arriver à une taille de $2^{\text {m }} 50$ et même un peu au delà. Sans être aussi redoutables, il est vrai, que des Crocodiles de même dimension, ils déploient cependant une force et une agilité extrềnes dans la poursuite de leur proie.

"La plupart des espéces, lisons-nous dans le texte allemand du zoologiste de Leyde, sont positivement amphibies, puisqu'elles ont été observées tantôt dans les lieux secs, tantôt dans les eaux. Il y en a peu qui habitent les sables et les déserts où elles vivent dans des trous; celles-ci ne vont jamais 
dans le voisinage des eaux et ne montent jamais sur les arbres. Elles se signalent surtout par leurs couleurs pâles."

“ Les belles espèces souvent si bien peintes, habitent presque tontes le bord des rivières ou même les côtes maritimes, mais parfois au si on les a trouvées au milieu des forêts presque inexplorées et loin des eaux douces ou salées. Elles grimpent avec une grande légèreté sur les buissons et sur les arbres, nagent trés-habilement et poursuivent ainsi dans les eaux, comme sur la terre, leur proie qui consiste en mammiferes, en oiseaux et insectes, ou bien en animaux aquatiques, tels que batraciens, poissons et crustacés. On les voit souvent guetter sur le rivage et saisir les animaux rejetés par la mer. Il n'est pas rare qu'ils approchent des demeures de l'homme, pour dévorer toute sorte d'ordures et des débris d'animaux. Comme ils recherchent avec ardeur les oiseaux, ils se glissent jusque dans les poulaillers et deviennent ainsi les ennemis redoutables des volailles domestiques. Ils sont eux-mêmes fréquemment attaqués par les crocodiles. "

Voici la liste des Varans admis par M. Schlegel; ils sont au nombre de quatorze, dont cinq, signalés par des caractères italiques, ont été décrits depuis la publication de l'Erpét. génér. Des espèces inscrites dans cet ouvrage comme distinctes (Var. ocellatus, Picquotii, albigularis et Bellii) ne représentent, selon le zoologiste hollandais, que de simples variétés.

Gen. MOXITOR

1. M. scincus Gr. (Merr.)

2. M. examihemalicus Schl.

Varielales :

Q. M. exanthematicus Schl, - Yaranus ocellatus Rüpp. Afr. sept.

U. X. cxauthem.: indicus Schl. - M. Aavescens Gr. (V. Pirquolii D B.).

c M. exantlem : capensis Schl_-Tup. albigularis Dand.

1. M inornatux Sél

5. $M$ tristis sichl. (Odatria punctata Gu.?

ร. M. Timnriensis $6 \mathrm{r}$.

6. M. Cepedianus Schl. (Tun. iudicas, - gutatus, I.engalensis Daud.).

7. M. nebulatus Gr. (Cuv.)

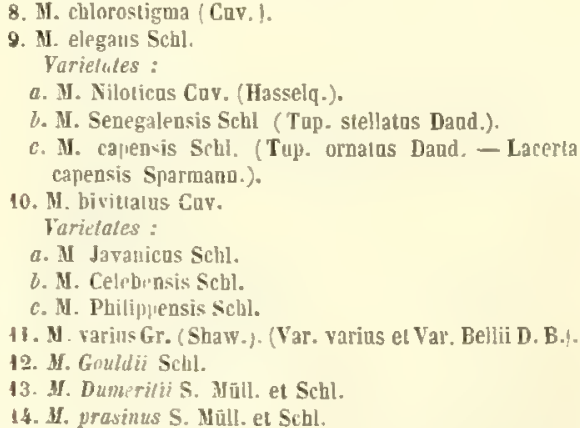

Parmi les cinq espèces qu'il faut mentionner ici comme nouvelles, il y en a deux seulement au Musée de Paris : c'est le Var. de Gould, Schl., qui prend rang après le Var. bigarré ( $\mathbf{n}^{\circ} 9$ bis) et le Vur. sombre (Mon. tristis), Schl., dont M. Gray admet l'identité avec celui qu'il a nommé Odatria punctata, identité que M. Schlegel lui-mème considère comme probable.

La place naturelle de ce second Varan est indiquée par la forme de la 
queue peu comprimée; c'est donc probablement, ainsi que les $V$ ar. du désert et de Timor, un Saurien, dont les habitudes sont celles d'un animal terrestre.

XIX. - 2 bis. Varan ponctué, Varanus punctalus, Dum.

Odatria punctata, Gray, Ann. nat. hist., t. II, p. 394; Grey's Trav. Austr., t. II, p. 422; Cat.of Liz., p. 7, et Zool. of the voyage of Erebus and Terror, p. 2, pl. I. - Monitor tristis, Schlegel? Abbildungen neuer Amphib., p. 73. - Varan ponctué, Cat. des Rept. du Mus. de Paris, p.49. - Id., Répert, in Erpét. génér., Dum. et Bib., t. IX, p. 256.

Téte petite; ourerture des narines allongée, presque à égale distance de l'angle antérieur de l'wil et de l'extrémité du museau, qui est légèrement obtus; queue à peine comprimée, à écailles épineuses et verticillées, mais sans crête semblable à celle des Varans aquatiques et portant, de chaque côté de sa base, un petit amas d'écailles épineuses peut-être spéciales aux máles.

Les écailles sus-orbitaires sont granuleuses, égales entre elles; à la gorge et au thorax, elles sont plus petites qu'au ventre, ou elles sont deux fois aussi longues que larges.

Le système de coloration de l'exemplaire unique du Musée de Paris est très-sombre, comme l'est celui du type d'après lequel M. Schlegel a établi l'espèce nommée par lui Monitor tristis. La description qu'il en donne convient à notre spécimen, car on peut dire, de même, que sa teinte générale est un brun noirâtre, qui passe tout à fait au noir sur la queue, dont la base est, ainsi que le dos et les pattes, ornée d'un petit nombre de taches jaunâtres, éteintes. Le ventre est beaucoup moins foncé, mais il est traversé par des bandes obscures. Les couleurs, au reste, peuvent être plus claires; on en a la preuve par les détails suivants empruntés à la description de M. Gray, qui la donne comme se rapportant plus spécialement à l'âge adulte. D'un vert olive, dit-il, avec des lignes noires étroites, réticulées, dont les entre-croisemenls limitent des espaces de forme hexagonale; tête, membres et queue noirâtres, portant des lignes transversales foncées et un petit nombre de taches d'une nuance plus vive. - Le Musée de Londres renferme, en outre, deux jeunes individus, l'un desséché, noirâtre, avec un rang transversal de taches ocellées, l'autre conservé dans la liqueur, et d'un vert sombre, relevé par des anneaux blancs disposés en travers sur le dos; la tête est finement piquetée de blanc, ainsi que les reins. Ūn très-jeune animal enfin, porte de nombreuses bandes étroites sur un fond gris.

Ce Varan, comme le fait observer M. Schlegel, présente dans son ensem- 
ble une certaine analogie avec le $V$. de Gould, mais ce dernier a les narines plus rapprochées de l'extrémité du museau, qui d'ailleurs est plus allongé. La queue du $V$. ponctué est moins comprimée, et ses écailles sont plus carénées. - L'obliquité des narines et leur situation près des yeux, chez le $V$. du désert, puis leur forme presque circulaire dans le $V$. de Timor, peuvent servir à faire distinguer ces deux derniers du $V$. ponctué. — On peut, en outre, tirer du système de coloration et de la différence d'origine de bons caractères pour éloigner les unes des autres ces trois espèces à queue cylindrique ou faiblement comprimée et qui, par cette conformation même, semblent être appelées à vivre dans les lieux secs.

C'est dans la Tasmanie et dans la province de la Rivière des Cygnes (Australie), que M. J. Verreaux a recueilli l'exemplaire unique du Musée de Paris et celui qui appartient au Musée de Leyde. Ceux du Musée britannique ont été reçus de différents points de l'Australie.

\section{XX. - 9 bis. VARAN DE Gould, Taranus Gouldii, Dum.}

Monitor Gouldii, Schl., Abbildungen neuer Amphib., p. 78. - Hydrosaurus Gouldi, Gray, Ann. nat. hist., t. I, p. 394, et Grey's trav. Austr., t. II, p. 422. - Varan de Gould., Dum., Cat.des Rept. du Mus. de Paris, p. כ2.

Narines situées près de l'extrémité du museau, qui est effilé; queue comprimée, surmontée d'une petite crête formée par deux rangées longitudinales d'écailles plus grandes que les autres; granulations sus-oculaires très-fines, serrées, et par cela mème bien distinctes de celles qui occupent les autres points de la région sus-céphalique; de chaque coté du cou, deux raies jaunes longitudinales.

Les parties supérieures, sombres chez deux de nos individus et à peine relevées par quelques traces jaunes sur la queue et sur les membres postérieurs, sont, chez les deux autres sujets, d'un noir brun entremêlé d'un grand nombre de petites taches jaunes qui, sur un animal d'âge moyen, constituent des bandes dorsales irrégulières, mais ces bandes sont d'une régularité parfaite autour de la queue. Les particularités ordinaires de ce système de coloration consistent dans la présence : $1^{\circ} \mathrm{d}^{\prime} \mathrm{un}$ trait jaune sur le bord saillant de l'orbite et se prolongeant plus ou moins sur le cou; $2^{\circ} d^{\prime}$ 'une tache noire allongée, située derrière l'oeil; $3^{\circ}$ d'une large bande jaune qui, partant du museau, passe sur le bord supérieur de l'ouverture de l'oreille, et va se perdre sur les còtés du cou; $4^{\circ}$ enfin, d'une bande noire qui, après avoir suivi 
la lèvre supérieure, se continue sur le cou parallèlement à la précédente. Les flancs sont tachetés de noir, et les parties inférieures sont d'un brun jaunâtre uniforme.

Si l'on compare ce Varan aux espèces de l'Océanie, on trouve des différences notables. Ainsi : $I^{\circ}$ le $V$. ponctué a la queue cylindrique, les narines à égale distance environ de l'angle antérieur de l'oeil et de l'extrémité du museau, qui est moins effilé; le système de coloration d'ailleurs n'est pas le mème; $2^{\circ}$ outre l'aspect tout particulier de sa robe semée de points jaunes, le $V$. chlorostigme a les orifices des narines arrondis et les plaques sus-orbitaires de dimensions inégales; $3^{\circ}$ le $V$. bigarré a, comme le précédent, les ouvertures des narines circulaires; les écailles des régions supérieures sont très-petites, et de plus, il y.a, sur le cou et sur le dos, alternance régulière de bandes transversales, les unes d'un noir profond, les autres formées par de gros points jaunes; $4^{\circ}$ chez le $V$. de Bell enfin, les trous extérieurs des fosses nasales sont ronds et rapprochés de l'extrémité antérieure du museau, et le système de coloration est remarquable par l'opposition des teintes brun-jaunâtre et noire, disposées sous forme de taches ou de bandes transversales.

Ces cinq espèces ne sont pas les seules qui aient été recueillies dans l'Océanie; M. Schlegel en a décrit une sixième sous le nom de Monitor inornatus (Abbild., p. 72). Elle est inconnue dans les Musées de Londres et de Paris. Elle tient de plus près aux Varans terrestres qu'aux Varans aquatiques.

Enfin, je ne puis également que nommer une septième espèce océanienne, décrite par MM. S. Müller et Schlegel dans le grand ouvrage publié par la commission scientifique de la compagnie néerlandaise des Indes Orientales Terhandling over de natuurlijke geschiedenis der Nederl. overzeesche bezitingen door de leden der natuurkund. Commiss. in Oost.-Indie en andere schrijuers Repı. p. 42, pl. v). C'est le Monitor prasinus recueilli dans la Papouasie ou Nouvelle-Guinée, et facile à distinguer de tous ses congénères par l'éclat de sa belle robe verte. Il a des formes délicates et sveltes, et une 'queue extrêmement longue.

L'ouvrage hollandais que je viens de citer, contient, en outre (pl. vi), la figure d'un autre Varan accompagnée d'une description ( $p$. 44) due aux mèmes zoologistes qui ont fait à mon père l'honneur de nommer ce Saurien le Bornéo Monilor Dumerilii. Le corps est ramassé, mais le cou est allongé, ainsi que la queve et la teinte générale est un brun presque uniforme. 


\section{CINQUIÈME FAMILLE : IGUANIENS OU EUNOTES.}

Le naturaliste qui étudie les types les plus remarquables des neuf grandes familles dont l'ordre des Sauriens se compose, saisit aisément les différences qui les distinguent. Ainsi, les quatre groupes que j’ai déjà passés en revue dans ce mémoire et dans le précédent offrent des caractères qu'il est inutile de rappeler ici, mais tellement tranchés que nulle confusion ne peut avoir lieu quand l'examen porte sur un Crocodilien, sur un Caméléon, sur un Geckotien ou sur un Varan. Il en est de même pour la famille des Amphisbéniens ou Glyptodermes à téguments non écailleux, mais annelés et divisés en petits compartiments quadrilateres, un peu saillants comme des tubercules réguliers, et pour la famille des Scincoídiens ou Lépidosaures, à écailles semblables sur toutes les parties du tronc et analogues par leur arrangement et par leur aspect à celles des poissons. Les trois antres familles sont trèsdistinctes des six précédentes; mais tandis que celles-ci offrent entre elles les dissemblances les plus frappantes, il faut pour les Lacertiens, les Chalcidicns et les Iguaniens, recourir à l'examen de particularités de structure un peu moins notables, mais cependant très-faciles à observer. Si, en effet, la tête des Lacertiens, comme celle des Chalcidiens, est protégée par des écuscons squameux ou plaques polygonales, et si la région ventrale, dans ces deux groupes, est revêtue de grandes écailles carrées, il faut, d'un autre côté, tenir compte de la disposition verticillée de toutes les écailles du tronc et de la queue chez les Chalcidiens nommés aussi, par ce motif, Cyclosaures. Leur sillon latéral, qui manque dans un petit nombre d'espèces seulement, et le peu d'extensibilité de leur langue sont de bonnes marques distinctives. Chez les Lacertiens ou Autosnures, c'est-à-dire vrais Lézards, les écailles, au contraire, ne sont pas verticillées; jamais on ne voit un sillon le long des flancs, et enfin la langue, quelquefois très-échancrée, est le plus ordinairement fort extensible. - Quant aux Iguaniens, on en reconnait le plus grand nombre à la présence d'une carène ou d'une crête dorsale plus ou moins développée, d'où le nom d'Eunotes. Deux autres caractères essentiels se rencontrent toujours: $1^{\circ}$ la tête est revêtue d'écailles plus ou moins semblables à celles du tronc et non pas de plaques polygones; $2^{\circ}$ la langue est épaisse, papilleuse, 
non engainée dans un fourreau, et son extrémité seule est libre de toute adhérence.

Ainsi caractérisés, les Sauriens fort nombreux rapportés à cette famille constituent un groupe très-naturel, dont les affinités zoologiques ont été assez complétement démontrées dans le t. IV de l'Erpét. génér. (p. I-5), pour qu'il soit inutile d'y revenir ici. Je m'attacherai seulement à faire voir l'heureuse application qui peut être faite à l'étude de ces Reptiles d'un mode spécial de classification signalé d'abord par Cuvier pour les Mammifères marsupiaux, et mis si habilement en oeuvre par M. Isid. Geoffroy SaintHilaire, pour les deux premières classes du règne animal, que le nom de ce savant professeur ne pourra plus désormais être séparé de la dénomination par laquelle on désigne cette méthode particulière. Je veux parler des classifications par séries parallèles, qui ont pour but de mettre en évidence les affinités naturelles avec plus de précision qu'on ne peut le faire dans un classement en série linéaire continue. Les développements de cette proposition m'entraineraient beancoup trop loin. Je les ai d'ailleurs déjà présentés dans un travail publié en 1854 (Rev. de Zool., nº 9, p. 467 et 544, Essai d'applicat. à la classe des Rept. d'une distribut. par séries paral.), où j’ai donné quelques exemples de l'emploi qui peut être fait avec avantage de cette méthode dans la classification des Reptiles. De ces différents exemples, je ne citerai que celui qui est fourni par la famille des Iguaniens, et qui est trèsconvenable pour démontrer l'utilité de cette nouvelle manière de considérer les rapports des ètres entre eux, rapports dont l'expression la plus approchée doil ềre l'objet constant des efforts du naturaliste.

Les genres nombreux compris dans cette vaste famille forment deux groupes qui, dans plusieurs classifications, constituent deux familles distinctes: celle des Iguaniens proprement dits et celle des Agamiens. Ces groupes sont cependant unis par des lieus assez étroits pour qu'il y ait lieu de les considérer comme ne représentant que leux sous-familles. On retrouve, en effet, dans chacune de ces deux divisions tous les caractères généraux propres aux Sauriens qui, ne pouvant rentrer dans aucune des huit familles autres que celle dont il s'agit, sont, par cela même, des Igraniens. Il faut seulement noter une différence anatomique relative au mode d'implantation des dents, car chez les uns, elles sont reçues dans un sillon creusé à la face interne de la mâchoire, qu'elles dépassent par leur extrémité supé- 
pieure, et contre laquelle elles s'appuient comme une palissade appliquée le long d'un mur peu élevé. Ce sont, suivant l'expression proposée par Wagler, des Pleurodontes. Chez les autres (Acrodontes, Wagl.), les dents sont fixées sur le bord libre des mâchoires, dans la substance osseuse, et elles y adhèrent par la base de leurs racines. Ceux-ci, en outre, n'ont jamais de dents palatines, contrairement à ce qui se remarque dans la plupart des Pleurodontes. Enfin, ces derniers, sauf une seule exception pour le genre Brachylophe, sont tous originaires du Nouveau-Monde, et tous les Acrodontes vivent sur l'Ancien-Continent. Or, malgré ces différences, on ne peut méconnaître les nombreuses affinités naturelles de tous ces Reptiles, quand on voit certaines formes se reproduire exactement dans chacun des deux groupes dont l'un semble, pour plusieurs des genres qu'il comprend, être en quelque sorte la répétition de l'autre groupe. De là naît la difficulté d'un classement convenable de cette famille en une série linéaire continue où l'énumération des genres Pleurodontes étant présentée la première et dans l'ordre le plus naturel, celle des Acrodontes vient à la suite et dans le même ordre. D'un semblable arrangement, il résulte que les animaux dont les analogies sont le plus frappantes sont précisément ceux qu'on éloigne le plus les uns des autres. Si, en effet, dans deux séries $a, b, c, d$ et $a^{\prime}, b^{\prime}, c^{\prime}, d^{\prime}$, les termes homologues sont exprimés par la même lettre, on voit qu'en les énonçant dans l'ordre unisérial, le terme $d$ se trouve suivi du terme $a^{\prime}$, qui a le moins de rapports avec lui et qui se trouve lui-même porté fort loin du terme $a$ son correspondant. Transformez cette série unique en deux séries parallèles où vous pourrez placer sur une même ligne horizontale les termes dont il importe d'exprimer les vraies affinités, multipliez les séries si cela est nécessaire, et les difficultés dont il vient d'ètre question disparaissent anssitòt, car la disposition suivante:

$\begin{array}{lll}\mathrm{a}, & \mathrm{a}^{\prime}, & \mathrm{a}^{\prime \prime} \\ \mathrm{b}, & \mathrm{b}^{\prime}, & \mathrm{b}^{\prime \prime} \\ \mathrm{c}, & \mathrm{c}^{\prime}, & \mathrm{c}^{\prime \prime} \\ \mathrm{d}, & \mathrm{d}^{\prime}, & \mathrm{d}^{\prime \prime}\end{array}$

indique, d'une façon très-nette, quels sont dans ces séries, construites chacune, comme cela doit ètre, suivant un ordre sérial continu, les homologies dont il faut tenir compte dans l'expression si essentielle en zoologie des affinités naturelles des êtres entre eux. 
ORDRE DES SAURIENS. - IGUANIENS.

Une revue rapide des genres comparables dans l'une et dans l'autre sousfamille des Igruaniens rend facile la démonstration des avantages de ce mode de classement. Mon père et Bibron, sans y insister beaucoup, ont cependant appelé l'attention sur ce sujet, par la construction d'un tableau inséré t. IV, p. 44 et 45 , où ces Sauriens, partagés en neuf tribus, sont rangés de façon que l'observateur saisit d'un coup d'oil les analogies des genres appartenant soit à la sous-famille des Pleurodontes, soit à celle des Acrodontes. C'est d'après l'étude attentive de ce tableau et guidé par les considérations si justes émises par M. Isid. Geoffroy Saint-Hilaire dans sa grande Hist. nalur. génér. des règnes organ., t. I, p. 416-482, sur ce point délicat et important de zoologie, que j’ai essayé de montrer, dans le Ménoire cité plus haut, les heureux résultats qui peuvent être obtenus de cette méthode ingénieuse de classification dans l'étude des différents ordres de la classe des Reptiles.

N'arrêtant donc plus spécialement ici sur la famille des Iguaniens, je fais d'abord observer que certains groupes peuvent ne pas avoir et en réalité n'ont pas leurs homologues. Tels sont, par exemple, parmi les Pleurodontes, les Anolis auxquels ne correspond ancun genre dans l'autre famille, car ils présentent seuls à l'antépénultième phalange de chaque doigt le singulier élargissement qui leur permet de se suspendre, même contre leur propre poids, aux corps les plus lisses et y rend leur ascension si facile. Tels sont encore, et pour ne citer que les exemples les plus saillants: $\mathrm{I}^{\circ}$ dans le Nouveau-Monde, les Corytophanes à tête de caméléon, prolongée en arrière par une longue apophyse osseuse, soutenant une crête cutanée plus ou moins étendue sur la nuque et sur le dos; $2^{\circ}$ dans l'Ancien-Continent, les Lézards volants ou Lragons, le lézard à collerette dit Chlamydosaure de King et l'Arpéphore à prolongement falciforme du museau que j'ai décrit (Cat. Rept. du Musée de Par., p. 92, puis Rev. de Zool., 1851, p. 213). - On peut, au contraire, mettre exactement en regard dans la série des Pleurodontes d'une part, et dans celle des Acrodontes de l'autre, et en démontrant ainsi leur parallélisme, les genre Basilic et Istiure, à cause de leur haute crête dorsale et caudale; le Brachylophe, le seul de sa sous-famille qui vive dans l'Inde et dans les îles de l'Océanie, et les Galéotes; l'Ophryesse et les Lophyres. Tous ces Sauriens ont le corps comprimé et surmonté d'une crête dont le développenent est variable. - Parmi ceux à tronc déprimé ou à peu près cylindrique, les Pleurodontes dits Léiosaures, Proctotrétes et 
Tropidolépides ont pour homologues dans l'autre sous-famille les Léiolépides, les Grammatophores et les Agames. Au nonveau genre américain, caractérisé par son tympan caché et nommé Holbrookia, on peut opposer les Phrynocéphales. Aux Phrynosomes de l'Amérique du Nord et d'une structure si bizarre, répond le Moloch de l'Australie, à corps non moins déprimé et encore plus hérissé d'aiguillons. Enfin, chez les espèces à queue épineuse, la répétition des mèmes formes dans chacun des deux groupes est très-évidente et fort remarquable. Il est facile de s'en assurer en comparant le Sténocerque, le Strobilure et le Trachycycle de l'Amérique du Sud aux Stellions de l'Ancien-Monde, puis les Pleurodontes nommés Oplures et Doryphores aux Fouette queiles de la seconde sous-famille.

Après ces citations, il me semble superfu d'insister davantage sur les utiles ressources que le classement par séries parallèles fournit au zoologiste qui cherche à exprimer, d'une manière moins imparfaite que par l'ordre unisérial, les rapports naturels des animaux entre eux.

- Je dois maintenant exposer les changements survenus dans la distribution méthodique des Iguaniens depuis l'année 1837 , où parut le t. IV de l'Eipét. génér., qui donne, jusqu’à cette époque, un historique complet de toutes les tentatives plus ou moins heureuses des classificateurs.

Le premier onvrage que j’aie à analyser est celui que M. Fitzinger a publié en 1843, sous le titre de Systema Reptilium. - Pour exposer sa classification, il faut rappeler encore une fois qu'il divise les Reptiles, soit d'après ses propres vues, soit d'après celles des zoologistes qui l'ont précédé, en 5 grandes séries: I Amblyglossa, Fitz.; II Leploglossa, Fitz. (Wiegm.); III Testudinata, Oppel; IV Dipnoa, Leuckaert; V Rhizodonta, Fitz.

C'est dans la première série que les Iguaniens prennent rang. Ils sont distribués dans les deux premiers ordres de cette série: Dendrobalce, Wiegm., et Humivaga, Id. Quant au troisième ordre (Ascalabole, Wiegm. [Schneid]), je m'en suis précédemment occupé.

Le premier ordre (Dendrobatce) comprend 2 sections: $1^{\circ}$ Acrodontes, Wagl., divisés en 2 tribus: Rhiptoglossa (Chamcleontes), Wiegm., et Pachyglossa, Wagl.; $2^{\circ}$ Pleurodontes, Wagl., offrant une semblable division en 2 tribus: Thoracopleure, Fitz, et Gastropleura, Id. - Le second ordre (Humivagae) est partagé en 2 sections : I ${ }^{\circ}$ Prosphyodontes, Wiegm.: $2^{\circ}$ Empleyodontes, Id. 
L'espace me manque pour présenter, ainsi que je l'ai fait en parlant des Geckotiens, la classification de M. Fitzinger sous forme de tableaux synoptiques mettant en évidence les caractères d'après lesquels il a établi parmi les Iguan. et les Agam. 2 I fam. et 6 r genres subdivisés en 85 sous-g. où sont comprises 200 espèces, dont 5 fossiles marquées d'un double astérisque ${ }^{* *}$. Je me borne à donner une liste complète de toutes ces divisions en indiquant par des caractères italiques les espèces non admises dans le t. IV de l'Erpét. génér., ou qui étaient inconnues quand ce volume a paru. Il y en a 44. Dans ce nombre, on en trouve 14 récemment décrites, 2 par M. Berthold, 12 par M. Fitzinger, et elles portent chacune un astérisque* ILes 3 o autres avaient été déjà signalées par différents zoologistes. Les motifs qui ont engagé M. Fitzınger à adopter ces dernières sont les mêmes que ceux dont j’ai parlé (p. 445 et 446) à propos des Geckos; je ne reviendrai donc pas sur celte discussion. - Voici cette liste.

\section{CLASSIFICATION DE M. FITZINGER. - 1843.}

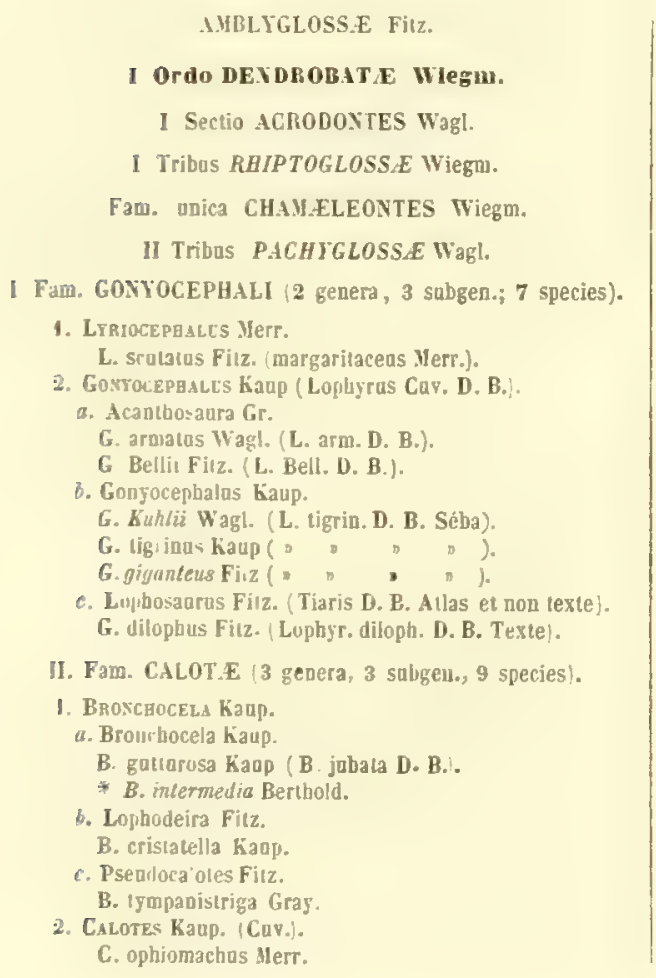

G. Tiedemanii Fitz. (C. versicolor D. B.).

C. Rouxii D. B. - C. mystaceus D. B.

3. Cеntтophon Gray.

C. Stodartii Gray.

III Fam. SEUIOPHoRi (2 gen., 2 spec.).

1. Srytophorus Wagl. (Sitana Cuv.)

S. ponticerianus Was!. (Cuv.).

2. Chlamrousantis Giay,

Ch!. Kingii Gray.

IV Fam, otocripte 1 gen., $\mid$ spec.)

1. 0tocruptis Wiem.

0. Wiegmanni Wagl. (bivittata Wiegm.).

V Fam. LOPHUR $(2$ gen., 2 subgen., 4 spec.).

1. Lopeura Wagl. (Gray) (Isturus Cav.).

a. Istiurus Cuv.

L. Lesueui ii Gray.

b. Lophura Fitz. (Gray)

L. amboinensis Gray. (Schlosser).

L. pustulosa Wagl (I. amboin. D. B.?).

2. Peysignathus Cuv.

Pl. cochunhiuensis Wiegm. (Ist. physign. D. B.)

VI Fam. DRACoNES (2 gen., 4 sulgen., 8 spec.)

1. Draco Wiegm. (Lin.).

a. Rhacodracon Filz.

D. finbriatus Kulit.

b. Draco Fitz (Lin)

D. viridis Daud. (D. volans Lin., D. Daudinii D. B.)

D. quinquefaseiatus Gray - D. Timoriensis Péron.

c. Pterosaurus Filz.

D. Dussumieri D B

d. Pleuropteras Fitz.

D. hæmatopogon. Boie. 
2. Drscostuĩds Fitz. (Dracnuculus Wiegm.). D. lineatus Daad.-D. personalus ( spilopterus) Wiegm.

\section{Sectio PLEURODONTES Wagl.}

I Tribus THORACOPLEURA.

I Fam. CORYTOPHANE (1 gen., 2 subgen., 2 spec.).

1. Corttophanes D. B. Boiel

a. Chamæleopsis Wiegm.

C. Hernandesii Gr. (Wiegm.) (C. chamæleopsis D. B.).

b. Corytophanes Boic.

C. cristatas Boie.

II Fam. HYPSILophi (6 gen., 17 subgen. 28 spec.)

7. Basiliscus Wiegm. (Laurenti).

c. Basiliscus Laur.

B. mitratus Dard.

3. Corsthæulus hanp.

B. vitlatus Wiegm.

** 2. Hylosit'ros Mantell (fossile)

* B. Mantelli Fitz.

3. Hүрsilophes Fìlz. (Wagl.).

a. Aloponotus D. B.

H. Ricordii Fitz. (D. B.)

7. Hetopoceros Wagl

H. cornutus Fitz. (Wagl.).

6. Hrosilophus Wagr.

H. nadicollis Fıtz. (Cav.) - H. rhinolophas Tiegm.

H. iguana (uberculatus) Wagk,

d. Amblyrbynchas bell.

H. cristatus Fitz. (Bell.). - H. ater Fitz. (Gray).

e. Conolophas Fiiz.

H. Demarlii Filz. (D. B.)

f. Brachylophus Car.

H. fasciatus Filz, (Cuy.).

4. Crcuira Harlan.

a. Cyclara Wiegm

C. carinata Harl. Horlani D. B.

C. pectinata Wiezm.

7. Cienosaura Wiegm.

C. denticulata Wiegm. (acantlura D. B.)

C. arliculata Wiegm. ( , )

C. Shawil Wiegm. ( D D)

C similis Wiegm. - C. Bellii Wiegm.

\$. Hrpsibatus Fitz. (Wagl.)

6. Oplaryoesa Boie.

H. Baici Fitzo - H. superciliosus Filz. (Boje).

6. Dryophilus Fiz. (Euyalus Wagl. parL.).

H. Lilineatus Fitz. (D. B.)

c. Enyalus Wagh.

H. calenatas Filz. (En. rhombifer Tagh.)

H. margarilaceus Fitz. (Spix).

d. Hypsibatus Wagl. (Ljeramodon part, D, B.)

H. ambra Wigl. (Lin. (L. ochrocollare D. B. (Spix).

C. Uperanodon D. B

H. pictus Wagl, (Neuw.),

6. Letosalires D. B.

a. Prislidactulus Fitz.

L. fasciatus D'0rbigny.

b. Leiossuras Fitz.

L. Bellii D B.

III Fau. PTYchositri (2 gen., 3 subgen, 4. spec.).

A. Prychosiches Filz.

a. Ptyctinsarn Fitz. 'Hypsibatus part. D. B,
P. panctatus Fitz. (D. B, ).

b. Ptychopleara Fitz. (Hypsibatas part. D. B.)

P. plica Fitz. (Lin.)

c. Tritropis Fitz. (Tropidogaster D. B.

P. Blainvillii Fitz. (D. B.).

2. Megadactruds Fitz. (Callisaurus Blainv.).

II. draconoīdes Fitz. (Blainy.).

I Tribus GASTROPLELRA.

I Fam, PLelrosaliri ( 4 gen., 4 spec.).

** 1. Leptositrirs Fitz. (fossile).

* L- neptunius Fitz.

** 2. Plevrosacres Meyer (fossile).

* P. Goldfussii Never

* 3. Reacheosatics Meyer (fossile).

* R. gracilis Heser.

*ix 4. Poecilopledrox Eudes Deslongchamps (tossile

* P. Baclilandi Eudes Desl.

II Fam. Poly Chri ( 2 gen., 4 subgen., 12 spec.)

4. Polychres Car.

P. marmoratus Cav.

P. virescens Neuw; (marmoratus D. B

P. anomalus Wiegm.

2. LFasictus Fitz. (Wiegm.).

a. Lirostrophas D. B.

L. Tantieri Fitz. - L, nndalatus Wiegur.

L. Fitzingeri Wiegm.

b. Ecphymatotes Fitz.

L. acatirostris miegm.

c. Læmanctus Wiegu.

L. lougipes Wiegms.

d. Norons TVagl.

L. ancatus Fitz. ( $\mathbf{W a g l}$ ). $-{ }^{-} \boldsymbol{L}$. gracilis Fitz.

${ }^{*} L$. Misomanni Filz, - * L. Endlicheri Fitz.

III Fam. DACTYLOE (5 gen., 20 subgen., 28 spec.).

1. Psedoocanaren Filz. (Chanæleolis Coctean).

P. Cocteaui Fitz. (Anolis chameleonides D. B.)

9. Ctengerus Filz. (Anolis part. D. B.)

a. Semiurns Fitz.

C. Ricorail Fitz. (An. Ric. D. B.).

C. Cavieri Filz (An. velifer Gav.).

b. Euripristis Fitz.

C. eqnestris Fitz. (Cuv.)

c. Nicroclenus Fitz.

C. Edwardsii Filz. (Merr.).

d. Ctenonolar Fitz.

C. bimaculatus Fitz, $(\mathbf{A n}$, Leachii D. B.

3. Ptichorotes Filz. (Anolis part. D. B.)

a. Istiocercus Fitz.

P. cristatellus Fitz. (Cuy.)

b. Ptychonotas Fitz.

P. fastians Filz. (An. allicator D. B.)

P. Dutnerilii Fitz. (An. nurmoratos D. B.

c. Ennotus Fitz.

P. gracilis Filz. (Neuw.) (An. nasicus D. B.)

* P. nasulus Fitz.

d. Deirontyx Fıtz.

P. vermiculatus Filz. (Cocteau).

e. Trachycoelia Fiuz.

P. linealas Fitz. (Daud.)

f. Ctenodeira Fitz.

P. Richardi Filz. (D. B.) 
4. Dactrzos Mascl. (Anolis part. D. B.)

a. Tropidopilus Filz.

D. fusco-anata Fitz. (1) 0 b.

b. Xiphosuras Fitz.

D. chloro-cyana Filz, (D, B.)

Xiphocercus Filz.

D. Valenciennii Fitz. (D. B.)

0. Eudacty lus Filz.

D. Goudotii Filz, (D, B)

e. Dactsloa Fitz. (Wagl.)

D. lucias Fitz. (D. B.).

D. Wiegnami Filz. (D. læviventris Wiegw.)

D. punctata Fitz. (Daud.)

f. Heterolepis Fitz.

D. pulchella Filz. (D. B.)

9. Trachspilus Fiız.

D. Sృgræi Futz. (Cocteau)

h. Pristucercus Filz.

D. biporcata Wiegm, (An, chloro-cyanus D. B.)

i. Clenocercus Fitz.

D. caroliuensis Fitz. (Cuv.). - D. Schiedii Wiegm. : Gastrotropis Filz.

D. nebulosa Wiegm, (An. Sagræi Cocteau)

a. Heteroderata Fitz. (Acantbulis Goct.).

H. loysiamam Filz. (Coct.).

IV Fam. DRAC0NTURE (Anolis D. B. part.).

1. Dricostcra Wagt. (I gen., a subgen., A spec.).

a. Dracoutura Wagl.

D. mitens Wagl., vel refalgens Schl.

b. Dracontojisis Fitz.

D. Nibschii Wiegm, - *D. Bibronii Fitz.

D. Bertholdi Fitz. (D. 12-striata Berth.).

I Ordo ICMIVAgE Wtegn.

1 Sectio PROSPHYODOXTES Wiegm.

I Fam. HETEROTHOPIDES (3 gen, 7 subgen, 9 spec.).

1. StEIroxotrs Filz.

a. Leioceplualus Gray.

St. carinatus Filz, (Holotr. Herminieri D. B.)

b. Sieironolas Filz.

St. Sclureibersii Fítz. (H. microlophus Coct.).

Stenocercas D. B.

St. rosei-ventris Do Orb.

d. Strobilurus Wiegm.

St. torquatus Wiegm.

2. Heterotriopis Filz. (Tracbrycyclos D. B.)

a. Ophryocentron Fitz.

- $\boldsymbol{H}$ horrida Filz.

b. Heterotronis Fitz.

* $\boldsymbol{H}$. equestris Fitz.

c. Trachycycles Dum. et Bib.

H. marmoratos Filz. (D'Orb.)

3. Tropidtros Neuwied (Ecplymotes Cuv.)

T. torquates Neaw, $-T$. mictolepidolus Fitz.

II Fam. STEIRoLEPIDES (3 gen., 5 subgen., 34 spec.).

I. Steirolepis Fitz. (Microlophas D. B. Tropiduras Wiegm.)

S. wicrolophas Fitz. (Micr. Lessonii Var.A, D. B.).

S. heletolepis , (, D Var, B, ,

S. perutiana, $($, Var. CetD, , )

- S. carinicurda.

S. semileniata, (Spix)

- S. bartoria (Spix).
2. Ptrcaoderra Filz. (Proctotrèles ptychodères D. B. part.).

P. nigro-maculata Filz. (Wiegm.).

P. signifera Fitz (D. B.)

P. Wiegmanni F, (D.B.)-P. Filzingerii F. (D, B.).

3. LIOL.ents Wiegm. (Proctorretus D. B. part.)

a. Proctotretus D. B.

L. Nattereri Fiz. - L. marmoratus Gravenhorst.

L. pectiualus Filz (D. B.)

b. Leiodeira Filz. (Proct. Leiodères D, B. part.)

L. nxycephalus Wiegm. - L. tenais Fitz. (D. B.)

L. pictus Filz. (D. B.) - L. cyan gaster Fitz. (D. B.).

L. multimaculatus Fitz, (D. B.),

c. Liolæmus (Wiegm.).

L. olivacens Witem. ( $\mathrm{Pr}$, chilensis Var, A. Dam, Bib.).

L. chilensis W. (Less.) (Pi', chil. Var, B, Dum. Bib.).

$L$. unicolor Gravenhorst.

4. Scezoporus Wiegu. (Tropidolepis Cur.).

a Sceloporus Wiegm.

S. torquatus Wiegm. - S. formosus Wiegu.

$\mathrm{S}$. spinosus Wiegm. - $\mathrm{S}$, horridus Wiegm.

S. Bellii Wiegm. - S. aculeatus Wiegm.

S. unduldtus Wiegm. - \$. grammicus Wiegm,

S. microlepidotus Wiegm.

b. Tropidolepis Car.

S. variabilis Wiegm. - S. aneus Wiegm.

S. scalaris Wiegm.

5. Hoplurus Cuy.

H. Sebre D. B. (0. torquatus Cav.)

III Fam. DoRYPHorl (3 gen, 4 spec.).

1. Doryphorus Fitz. (Cuv.).

D. Maximiliani Fuz. (Opl. Max. D. B.)

2. Urocentron Kaup. (Doryphorus Cuv.)

D. Dandind Firz. (Doryph. azur. Cuv.), (Lin).

U. azureum Kaup. (Doryph. azur, Cuv.), (Lin.).

3. Hoplocencus Filz.

*

IV Fam. PHRYNosomatA (1 gen., 3 subgen, 5. spec.).

4. Phrryosoma Wiegm.

a. Phiynosouna Wiegm.

P. orbiculare Wiegm. - P. Douglasii Wiegm. (Bell).

b. Batrachosoma Fitz

P. coronatum Blainy

c. Tropidogaster Filz.

P. cornutum Gr. (P. Harlaní Wiegnt.)

P. buponium Wiegm. (P. Harlanii Wiegn.?).

II Sectio EMPHYODONTES Wiegm.

I Fam, TRAPLLI ( gen., 8 subgen., 13 spec).

1. Phrynopsis Fitz. (Agama Daud. part.).

a. Psamuoplílus. Fitz.

P. dorsalis Fitz. (Gr.)

6. Phrynousis litz.

P. atra Filz. (Dand.)

P. Savignyi Filz, (D. B.)

2. Podorrhos Fitz. (Agama Daud, part.).

a Podorrioa Fiz.

P. Luberculata Filz, (Gr.).

P. colonorum Fil\%. (Daud, )

b. Psendorrapelus Filz.

P. Sinaîta Fisz. (Heyden)

c. Planodes Fíz.

P. aglis Fitz. (Olivier).

d. Trapeloldis Fil.

p. grngrindeuta Filz. (Pallas) 


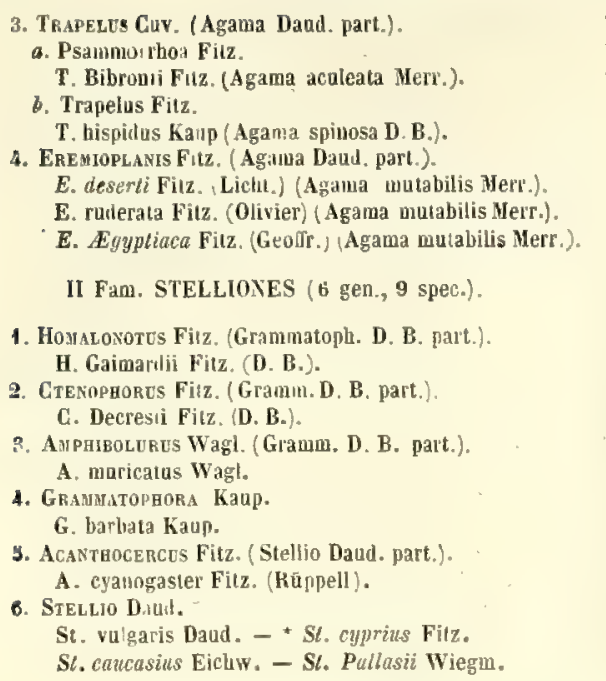

1. Cextrocencus Fitz. (Uromastix Merr. part.). C. Hardwickii Filz. (Gray).

C. similis Firz, (Ur. griseus Gur.).

2. Uromastix Merr.

U. ocellata Lirht. (ornatus Rüpp.).

U. viridis Wiegm. (spinipes D. B.).

U. spinipes Merr.

U. acant!inarus Bell.

3. Leiolepis Cuy.

L. gutlata Car.

IV Fam. PHRYYocePHALI (3 gen., 2 subgen., 6 spee.).

4. Saccostoma Fitz. (Phryn. Kanp part.). S. auritun Fitz. (Pallas).

2. Phiqnocephalus Kanp.

P. ocellatus Eichw. - P. caudivolvulus Fitz.

P. interscapularis Fitz. vel nizricans Eıchw.

3. HeLIoscopts Fitz.

a. Heliostonus Fitz.

H. uralensis Fitz. (Pallas).

b. Pltrynosaurus Fitz.

H. Olivieri Fitz. (D. B.)

- M. Gray, dans son Cat.of Liz., p. I 78-263, a décrit les Iguaniens pleurodontes et acrodontes; il les rapporte aux deux familles nommées Iguanida et Agamida, formant par leur réunion une tribu (Strobilosaura). La première famille renferme 54 genres et il y en a 34 dans la seconde. De même que pour la classification de M. Fitzinger, je me trouve dans l'impossibilité, par défaut de place, d'insérer ici des tableaux synoptiques résumant ce mode d'arrangement. Je me suis altaché, en dressant la liste suivante des 209 espèces (Iguanidie, г3o; Agamida, 79) rapportées aux 88 genres, à mettre en évidence, en les signalant par des caractères italiques, les 60 espèces qui n'étaient pas connues quand le t. IV de l'Erpét. génér. a paru, ou qui n'ont pas été admises dans cet ouvrage. Je comprends dans cette énumération les 3 genres et les 3 espèces que M. Gray a fait connaitre depuis la publication de son Cat., et qui portent ici les numéros 15 bis, 15 ter et 15 quater. D'autres espèces nouvelies, au reste, décrites plus ou moins récemment par ce même zoologiste, seront indiquées dans l'histoire des genres auxquels elles se rapportent.

On voit par la liste quisuit et par celle que j’ai extraite du Syst. de M. Fitz. combien les richesses des collections zoologiques, et en particulier dans le Musée britannique, se sont accrues depuis une vingtaine d'années, mais aussi combien ces naturalistes ont multiplié les coupes génériques en donnant souvent une trop grande importance à de simples caractères d'espèces. 


\section{CLASSIFICATION DE M. GRAY, 1845.}

Tribus IV. - STROBILOSAURA.

\section{Fam. - IGLANIDE.}

1. Polychrus Car.

P. marmoratus Cuv.

2. SpR.s.ups Gray (Polychrus park. Wiegm. D. B.)

S. anomalus $\mathrm{Gr}$. (Wiegm.)

3. Urostrophtis D. B

C. Vautieri D. B.

4. Ecphy yutes Fitz (uon Cuv.). (Læuanctus Wiegn. part.).

E. Fitziugerii Gr. (Wiegm.).

E. obtusirostris Gr. Wiegm.)

E. andulatus Gr. (W.). - E. acutirostris Fiı. (W.).

5. L.erasctes Wiegm. part.

L. longipes WViegat

6. Igcavi Laur.

I. talerculata Laur, - I. delicalissima Laur.

I. rhinoloptba Wiegm.

7. A Luposotes D. B.

A. Hirordii D. B.

8. Bracartopacs Cuy.

B. fascialus Cuv.

9. Netopoceros Wagl.

M. coruetus $\mathbf{W}_{\mathrm{agl}}$

10. Trachycephales Gr. (Amblyrhyncas Bell, parı.). T. subcristatus Gr. (Ambl. Denarlii D. B.).

11. Oreocephales Gr. (Ambl. Bell, part.)

0. crislatus Gr. (A. cristatus Bell et A. ater Gr.).

12. Crclera Hurlan.

C. Muc-Leayi Gro- C. Collei Gr.

C. nubila Gr. (C. carinara Harian).

13. CTenosadra Gr. (Cycl, Harl. part.).

Cl. acantiura Gr. (Shaw).

Cl. pectinata Gr. (Wiegm.).

14. Exrablosalics Gr.

E. quinquecurinalus $\mathrm{Gr}$.

1.. Basiliscus Laur.

B. americanus Laur. (B. mitratus Daud.).

15 bis. Ptesosatra Gi. (Ann. nal. hish., 1852, i. X, p. 438), P. Seemunni Gr.

15 ler. Lophusagra Gr. ( , , , , L. Goodriduii Gr.

15 qualer. Cristasauba Gr. ( " , ). C. milrellu Gr.

16. Corvtв Eutes Kaup.

C. viltatus Kaup. (Wiegm.).

17. TaYsasudactrul's Ge.

T. bilinealus $\mathrm{Gr}$.

18. Conytophanes Boie.

C. crislata Boie.

19. Chaneleopsis Wiegu,

Ch. Heruandesii Wiegm.

20. Exralies Wagl.

E. rhonbifer Wagl. - E. bilineatns D. B

21. Ophriroessa Buie.

0. superciliosa Boje.

22. Chaydeolis Cocieau (Auolis part.).

Ch. Feruandina Coct.

Archives du MusévH. T. VIII.
23. Xiprosurus Fitz. (Anolis part.)

X. velifer Gr. (Cuv.), $-\mathbf{X}$. cristatellus Gr. (Cuv.)

X. Risordiı Gr. (D. B.)

24. Dactrloa Gr. (Wagl.) (Anolis part.).

D. equestris Gr. (Guv.). - D. E.twardsii Gr. (Merr.).

25. Rhimosadros Gr. (Anulis part.)

B. gracilis $\mathrm{Gl}^{*}$, (Neuw.). (An. nascicus D. B.)

26. Axolius Cuv. part. (Anolis part.),

A. Leachi D. B. A. lineatnpus Gr.

A. occipitalis Gr. A. maculatus $\mathrm{Gr}$.

A. linealus Daud. A. pulchellus D. B.

A. Cepesii Merr. A. vermiculatus Coct.

A. Marmoratus D. B, A. stenodactylus Gr.

A. porcatus Gri. A. reticulatis $\mathrm{Gr}$.

A. principalis Gl'. A. fusco-auratus D'Orb.

(A. Carolinens. Cay.). A. renens Gr.

A. Aavesnens Gi: $\quad$ A. lucius $\mathbf{D}$ B.

A. Richardii D. B. A. Goudolii D. B.

A. Grahomi Gr. A punciatus Dad

A. punce. G. (now Daud.). A buliaris Lin.

A. nebalosus Gl (A. chloro-lyanus D. B.?) (A. Sagraei Coct.). A. Valeuciennit D. B.

27. Acantholis Coct. (Anolis part.).

Ac. Los siana Cuct.

28. Dracusura Wagl. (Anolis part.)

D. niteus Wagl. (A. refulgens Schl.)

D. chrysolepis Gr. (D. B.)

29. Nunors Wagl.

N. aluratus Wagl.

30. Tropidol Ers Cuv. (Sceloporus Wiegm.)

T. undulaus Cuv. T. grammicus Gl, (W.).

T. torquatus Gr. (W.). T. nuctolepid D. B. (W.)

T. formusus D. B. W.). T. variabilis D. B. (W.)

T. spinosu- Gr. (W.). T. aneus D. B. (W.)

T. hur ridus D. B. (W.). T. scalaris Gr. (W.).

31. Letodera Gr. (Léioderes D. B.). Tropidurus W. part. (Pluctulielus D. B. Part.)

L. chilensis Gr. (Less.). - L. gracilis Gr. (Bell.)

L. Gravenhorsiti Gr.

32. Leioleas Gr. (Wiegm.) (Froctotretus D. B.)

L. csauogaster Gl" (D. B.). L. tenuis Gr. (D. B.)

L. Bellii Gr. L. signifer Gl', (D. B.)

L. Bibronit Gr. (Bell.). L. maculatus $\mathrm{Gr}$.

L. lineatus Gr. L. Fucinueri G. (D.B.)

I. nigro-macalatus W. L. Darwinii Gr. (Bell.)

L. incouspicus Gr. L. Kingii Gr. (Bell.).

L. pictus Gr. D. B. $)$. L. Wirgiuannii Gr. (D. B. $\rangle$

33. Pi Ygoderus Gr. (Prolotretus D. B. part.).

Pt. pectinatus Gr. (D. B.)

34. Pructothetus D. B. (part.)

P. multimaculatus D. B.

35. LelucephaLUS Gl. LHolotronis D. B. Tropilluris Fitz. part.).

L. Herminierii G. (D. B.). L. Solureibersii G. (Filz)

L. mierolepis Gr. (H. micrulophus Coct.)

L. carinutis Gr. L. Grayi Bell.

L. Mac-Leuyii Gr. L. ornatus Gr.

36. STENocences D, B.

S. rosei-ventris D'0rbigny. 
37. Teachycreles D. B. T. nırmoratus D'orbigny

38. Taraguira Gr. (Eephymotes Cuv, noli Filz.). T. torquala Gr. (Nenw.).

T. Darwinii Gr. - T. Smithii Gr.

39. Microlophés D. B.

M. Peravianus Gr. (M. Lessonii D. B.).

20. Oplurus Car.

0. Brasiliensis Gr. (0. torquatus Cay. 0. Sebæ D. B.).

41. Strobilcres Wiegm.

S. Lorquatus Wiegm.

42. Uranscanos Kaup (part.) (Uperanodon 0: B.).

Ur. umbra Kaup. (Lin ), - Ur. pictom Kaup. (Neaty.).

43. Plica Gr. (Lin.) (Hypsibatus Wagl.)

P. umhra Gr. (Latr.) - P. punctata Gr. (D. B.)

44. Leiosalerts D. B.

L. Bellii D. B. - L. fasciatus D'0rbigny.

45. Diplol eves Bell

D. Darwinit Bell. - D. Bibronii Bell.

46. Troptocrus Neuw. (part.). Oplurus D. B. (Cuv.) part. T. torquatus Nenw.

47. URocentron Kaup (Doryphoras Cav.).

U. azoreun Kaup.(Lin.)

48. Perquatrous Gravenborst (Oplarus part.). Ph. Palluma Graveuhorst.

49. Callisaures Blainr. C. draconojdes Blailzy.

50. Tropinogaster D. B. T. Blainvillii D. B.

51. Phrunosoma Wiegni. P. Douglasii Wiegm. (Bell. ). - P. orbicalare Wiegm. P. Blainvillii Gr. (coronatum Blainv.)

P. cornatum Gr. (Harlanii Wiegm.).

\section{Fan. - AGAVIDE.}

1. Draco Lin. (part.)

D. volans Liu. (D. Dandinii D. B.).

D. Timoriensis Pérou, - D. fimbriatus Kubl.

2. DRacocella Gr

D. Dussnmieri Gr. (D. B.).

D. hrematopogon Gr. (Boie).

3. Dracuncules Wienm.

D. quainquefasciatus Gr. D. lineatus Daud.

D. orualus Gr. D. mnculatus G

D. spilopteras Wiegm.

4. SiT

S. Ponliceriana Cav.

5. Lirtocephalcs Metr.

L. margaritaceus Merr.

6. Ceratuphora Gi.

C. Stoddartii Gr.

7. Otocriptis Wiegm. 0. biviltata Wiegu.

8. Gongocephalt's Kaup (Loplyrus D. B. part.) G. chamæleontinos Gr. (Laur.). (Lac. tigrina Séba).

9. Dilophyous Gr. D. grandis $\mathrm{Gr}$.

10. Tiaris D. B. (Lophyrus D. B. part.).

T. megapogou Gr. (L. dilophus D. B.).

T. Bellii Gr. (D. B.). $-T$. Sophice Gr.

11. Acanthosacra Gr. (Lophyyus D. B. part.). A. armaia Gr. (Wagl.)
12. BrovennCELA Kaup.

B. cristatella Kaup. - B. celebensis Gr. (Schl.)

B. gulturosa Schl. (jnbata D. B.).

B. marmor. Houbr. Jacq. - B. Lympanistriga Gr.

13. Salea Gr.

S. Horsfeldii Gr.

14. Calotes Cav.

C. Maria Gr. - C. ophiomachus Ierr.

C. versicolor D. B. (Daud)

C. minor Gr. - C. Emma Gr.-C. Rouxii D. B.

C. mystaceus D. B.

15. Chelosania GE.

Ch. brunnca $\mathrm{Gr}$.

16. Charasia Gr. (Agama part.).

C. dorsalis $\mathrm{Gr}$

17. Gindalia Gr.

G. Bennettii Gr.

18. LOPHERA Gr. ( Istiuras Cav.).

L. Amboinensis Gr. (Schlosser).

L. Shawiî Gr. (Lac. lophara Shaw.)

19. Physignathos Cav. (Istiorus D. B. part.)

P. Cocincinus Guv. (Wiegm.) - P. Lesueurü Gr.

20. Chlamtooshurus Gr.

C. Kingii Gr.

21. HATTERU Gr.

H. punclata $\mathrm{Gr}$.

22. LopHognathus GT

L. Gilberliz Gr.

23. Diporophora Gr.

D. bilineata $\mathrm{Gr}$.

24. Gramitophora Kaup,

G. crislata Gr

G. barbala Kaup.

G. maculala G. (Gaimardi D. B.)

G. Decresii D. B. G. omala Gr.

25. LATDAKIa Gr. (Agama Dand, part.)

L. Luberculata Gl'.

26. STELlo Daud.

S. cordylina Gr. (Lawr) (S. vulgaris Daud.)

S. Cancasius Eichwald. - S. cyanogaster Rüppell.

27. Aguma Daud. part.

A. colonorum Daud

A. occipilalis Gr. (A. colon. Var: Dom, Bib.)

A. atra Daud. - A. agilis Olivier. - A. aculeata Merr.

A. hispida Gr. (spiusasa D. B. non Gray).

28. Trupeles Cuv. part. (Agama Daud. part.)

T. Sayigayi Gr. (B. B.)

T. ruderata Gr. (Olivier) (Ag, mutabilis Merr.)

T. sanguinolentus Eicbw. (I'allas)

T. Sinailus Gr. (Heyden)

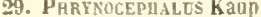

P. Olivieri D. B - P. heliosconus Kand. (Pallas).

P. caudivolvalus Fìtz. - P. Tickelii Gr.

30. Megalochilus Eichw. (Phrynoceph. Kaup part.).

M. anritus Eichw. ( Pallas)

31. Unoyastix Merr.

U. ornatas Rupp. - L. spinipes Merr.

U. acanthinurus Bell. - U. fasciatus Menestriès.

32. SaARA Gr. (Lromastix part.).

S. Hardwickii Gr.

33. Leiolepis Cay.

L. Bellii Gr. (guttatus Gav.). - L. Reevesii Gr.

34. Moloci Gr.

M. horridas $\mathrm{Gr}$. 
ORDRE DES SAURIENS. - IGUANIENS PLEURODONTES.

En I 850 , S. A. le prince $\mathrm{Ch}$. Bonaparte a présenté la liste suivante des Sauriens appartenant aux deux groupes des Iguaniens et des Agamiens (Conspectus system. Herpet. et Amphib., Leyde, tableau in- $\mathrm{f}^{\circ}$ ) :

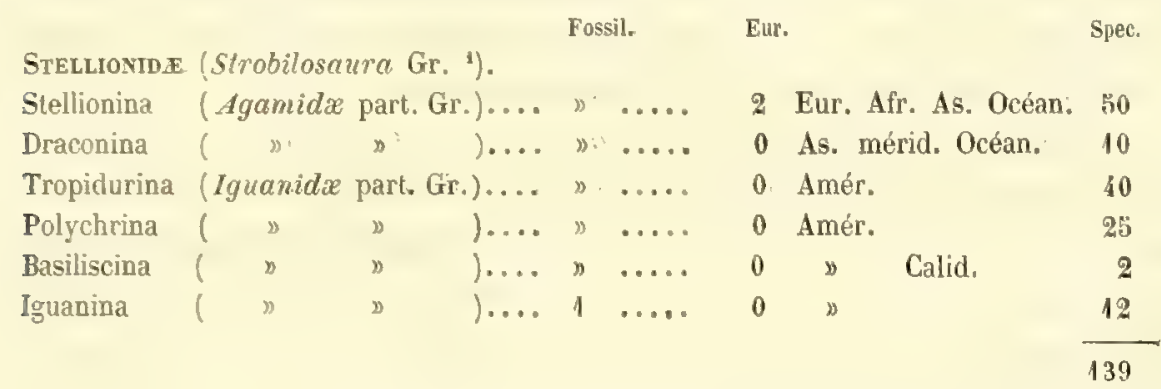

Les différences numériques relatives aux divisions établies par les classificateurs dont je viens d'analyser les travaux, et à celles qui ont été admises par les auteurs de l'Erpét. génér., peuvent se résumer' ainsi :

$\begin{array}{lccccc} & & \text { Familles. } & \text { Genres. } & \text { Sous-genres. } & \text { Especes. } \\ \text { 1837. Dum. Bib. Erpét. génér. } & 2 . & 46 . & 2 . & 152.2 \\ \text { 1843. Filz. Systema......... } & 24 . & 61 . & 85 . & 200 . \\ \text { 1845. Gray Catalogue........ } & 2 . & 88 . & 0 . & 209 . \\ \text { 1850. } & \text { Bonap. Conspectus..... } & 6 . & & & 139 .\end{array}$

\section{PREMIERE SOUS-FAMILLE DES IGUANIENS : LES PLEURODONTES.}

Les Sauriens appartenant à cette grande division ont pour caractère commun le mode d'insertion de leurs dents, qui sont fixées sur le bord interne d'un sillon creusé dans les machoires: Ce sont les Iguanes proprement dits ou Iguanides, si l'on veut employer ce nom par opposition au mot Agamides, qui sert souvent à désigner les Acrodontes ou Agames.

Quatre petits sous-genres, dans cettè première sous-famille, précèdent le grand genre Anolis. Je n’ai aucune observation à présenter sur le $3^{\circ}$ (UnoStropire; Dum. et Bib.), ni sur le $4^{\circ}$ (Norops Wagl.), qui comprennent chacun une seule espèce dans l'Erpét. génér. Il faut ajouter cependant que

1. Ce groupe est placé entre ces deux divisions : Gecconidæ et Chamæleontidæ appartenant tous trois à une mème tribu: Pachyglossi; cette tribu est la première dans le $6^{e}$ ordre (Saurii vel Lacertse) de la $3^{\circ}$ section des Reptiles: Squamata. Le tableau de classification dont il s'agit ne contient aucune indication sur les geures.

₹. En comprenant dans ce nombre bi espèces de Phrynocéphales ( $t$. IV, p. 54 3 ) non portées à la table de lÉrpét. génér. 
M. Fitzinger (Syst. Rept., I 846, p. 63) a donné les noms de trois autres espèces de Norops du Musée de Vienne:Lcemanctus (sous-genre Norops), gracilis, Wiegmanni, Enclichleri, originaires du Brésil.

Quant au $1^{\mathrm{ex}}$ genre, que Cuvier a nommé Polychre, j'ai à signaler une troisième espèce, décrite en 1845 par M. Berthold (Uber verschiedene neue oder seltene Reptilien aus Neu-Granada in Abhandlungen der Königlichen gesellschaft der Wissenschaften zu Göttingen, t. III, p. 5, pl. I, fig. 1). C'est le Polychrus gutturosus, remarquable par le développement des écailles de la région inférieure du cou, car elles sont une fois plus grandes que les autres. Ce Saurien ne se trouve pas au Musée de Paris qui, outre le Pol. marbré, possède maintenant des échantillons du Pol. anomal Wiegm., bien conformes à la description tracée par ce zoologiste.

Le $2^{e}$ genre que Wiegmann a établi sous le nom de Lamancte est presque inconnu dans les Musées; aussi doit-on s'en tenir aux détails qu'il a présentés d'après les types uniques du Musée de Berlin, et à ceux qui ont été fournis par Spix sur une espèce conservée à Munich (Polychrus (Lcm.) acuturostris) et recueillie au Brésil.

Nos collections possèdent cependant, depuis 1845, un beau lézard, qui est pour nous le premier spécimen de ce genre. 11 a été recueilli dans le Mexique, près de la ville d'Oaxaca, et donné par M. Ghuisbreght. D'après la comparaison de ce Reptile avec celui qui a été représenté par Wiegmann (Herpet. mexicana, pl. iv) : L. longipes, il n'y a pas de doute sur l'identité de l'espèce, mais certaines particularités sont en opposition avec quelques-uns des caractères attribués à ce genre. Ainsi, nous trouvons des dents palatines et une ligne saillante sur le milieu du dos, formée par la proéminence de la carène des écailles de cette région: d'où résulte l'apparence d'une crête longitudinale très-basse, particulièrement évidente sur la nuque à une très-petite distance de la tête; an delà, elle est de moins en moins apparente et cesse complétement à l'origine de la queue. La diagnose inscrite par Wiegmann dais son texte (p. 16, Gen. ro et p. 45) porte cependant qu'il n'y a pas de dents au palais et que la carène du dos est sans crète et sans dentelures. De plus, le spécimen dont il s'agit, comme le montrent les fig. 4, 4 a de notre pl. xxi, a, sur le bord postérieur de la tête prolongé en une sorte de casque plat et arrondi, onze à douze écailles saillantes triangulaires et pointues coustituant une sorte de cou onne. Rien de semblable ne se voit sur la pl. iv de 
l'Erpél. du Mexique; il y a néanmoins identité parfaite avec notre spécimen pour la forme de la région occipitale. Je me borne à mentionner ces faits observés sur un échantillon unique, et dont il est par conséquent impossible de déduire des conclusions applicables au genre tout entier, surtout en raison de la rareté singulière des Laimanctes dans les collections erpétologiques, car on manque de renseignements suffisants sur ces animaux.

T. AEBTE ANOLIS, ANORIS, DAUDIN.

Cette division générique, la plus nombreuse en espèces, comprend tous les Iguaniens à doigts plus ou moins dilatés au niveau de l'antépénultième phalange. Ce caractère est d'une grande importance dans la méthode naturelle, puisqu'il est l'indication manifeste d'une remarquable conformité dans le genre de vie. Il n'y a cependant pas une homogénéité tellement complète entre tous ces Sauriens, que des subdivisions n'aient pu être proposées pour ce groupe. On en a vu plus haut la liste dans les classifications assez récentes de MM. Fitzinger et Gray, dont j’ai simplement présenté un résumé, n’ayant point à discuter ici la convenance on l'inopportunité du démembrement d'un genre si naturel et si distinct que l'est celui des Anolis qui pourrait, par cela mème, devenir le type d'une sous-famille parmi les Iguaniens.

M. Berthold, en 1840 (Uber verschiedene neue oder seltene Amphibien arten in Mém. de l'Acad. de Göttingue, 1843, p. 62, pl. Ir, fig. 7 et 8), a décrit, avec la dénomination particulière de duodecim-striala, une espèce nouvelle à doigts peu dilatés et que, par ce motif, il a placée dans le genre Draconura, Wagl. (Dracontura, Fitz.), établi pour l'Anolis refulgens (vel nitens), genre non adopté par mon père et par Bibron, qui ont rapproché de cette dernière espèce celle qu'ils ont nommée Anolis chrysolepis, en les regardant l'une et l'autre comme types d'une subdivision parmi les Anolis 1. L'espèce de M. Berthold, Dracontura, 12-striaia (Diacontura Ber-

1. Une bonne représentation comparative des doigts à élargissement considérable ou peu marqué se trouve dans l'Atlas de l'Expéd. du comte de Castelnau, dans les parties centr. de l'Amér. du Sud. Rept, pl. Mr, fig. 2 a et $1 a$, tels qu'on les voit dans les An. nasique et chrysalépide. Voyoz, en outre, notre pl. xix, fig. 1, main de l'An. resplendissant. - Sur cette même pl. xix, fig. 2, $l^{\prime} A n$. de Valenciennes, si remaryuable par la petitesse des écailles ventrales, ce qui, d'ailleurs, le distingue de tous ses congénères, est représenté ru par dessous et amplifié, pour que le caractere puisse être bien saisi par l'observateur. 
tholdi, Fitz.) est inconnue au Musée de Paris. Elle se distingue facilement des deux précédentes en ce que les écailles médianes du dos, plus grandes que les autres et carénées, forment I 2 séries longitudinales au lieu de 5 ou 6, comme chez l'Anolis dit Dr. chrysolepis. Chez celui qui est nommé Dr. refulgens, il n'y a que 2 rangées d'écailles plus grandes et, en outre, elles sont lisses.

Relativement au Saurien inscrit par Wiegmann (Herpet. mex., p. 16), comme Dr. Nitzschii adopté par M. Fitzinger, qui en signale la présence au Musée de Vienne, l'opinion émise par les auteurs de l'Erpét. génér. qu'll ne devait pas différer de leur An. chrysolépide a été confirmée par Wiegmann Iui-mème, ainsi que M. Troschel nous l'apprend (Fauna von BritischGuiana Reisen, Rich., Schomburgk, note de la p. 649) ${ }^{1}$.

Nous trouvons indiqué, par M. Fitzinger, un autre Anolis reçu du Brésil, voisin sans doute des précédents : Dr. Bibronii, et M. Grosse. (Ann. and Mag. of nat. Hist., $2^{\mathrm{e}}$ série, t. v1, p. 346), signale une espèce originaire de la Jamaïque : Dr. catenata. - C'est près des Draconures que doit être placé un Saurien étiqueté par Wiegm. au. Musée de Berlin, An. planiceps, à en juger par la description donnée par M. Troschel (Faunu von Brilisch-Guiana Reisen, Rich. Schomburgk, p. 649).

- Avant de parler des Anolis nouveaux du Musée de Paris, je présente en note une liste, qui montre que, malgré ses richesses, il s'y rencontre cependant quelques lacunes par suite des découvertes récentes des naturalistes étrangers ${ }^{2}$.

1. J'ajouterai, à cette occasion, à propos de deux synonymies présentées par mon père et par Bibron dans l'histoire des Anolis, que M. Troschel, d'après l'examen des types mêmes de Wiegmann, considère, contrairement à co qui est énoncé dans leur Erpét. génér. sous forme dubitative, il est vrai, (p. 127 et 152), les Dactyloa bi-porcata et nebulosa comme distincts des An. Carolinensis et Sagrai.

2. An. occipitalis Gray (Ann. nat. hist., t. V, p. 112); An. porcatus Gr. (Id.); An. punctalus Gr., non Daud. (Id.); An. linealopus Gr. (Id.); An. maculatus Gr. (Id.); An. stenodactylus Gr. (Id.); An. veticulatus Gr. (Id.); An. æneus Gr. (Id.). An. flavescens Gr. et An. Grahami Gr. (Cat. of Liz., p. 202, où sont indiquées aussi les huit espèces précédentes). An. latifrons Berthold (Neue oder seltene Rept. aus Neu-Granada in Mém. de Göttingue, 1845-47, p. 6, pl. 1. fig. 2). An. iodurus, or purple tailed Gosse (Ann. of nat. hist., $2^{\mathrm{e}}$ série, t. VI, p. 344); An. opalinus Gosse (Id.); Placopsis ocellata Grosse ( $I l_{\text {. }}$ ). - Pour la détermination spécifique si difficile des Anolis, on peut se servir arec avantage du tableau synoptique de l'Erp.t. génér., t. IV, p. 90. Il faut ensuite tenir compte de l'origine et du système de coloralion qui, au reste, ne tarde pas à étre altéré par l'alcool. 
XXI. - 14 bis. Anolis a bandes trangversales, Anolis transversalis, A. Dum.

$$
\text { Pl. xix, fog. } 3 \text { et } 3 a \text {. }
$$

Idem, Id.,.Cat. méth. des Rept. du Muséum, p. s7. - Id., Répert. Erpét. gén., Dum. Bib., t. IX, p. 262. - Id., Guichenot (Expéd. parties centr. de l'Amér. du Sud, par M. de Castelnau).

Écailles ventrales plates, imbriquées, plus grandes que celles des flancs, qui sont aussí dilatées que les autres; cou et dos surmontés d'un petit pli de la peau sans dentelures; point de carènes en avant du front; de larges bandes brunes sur le tronc et sur la queue.

La tête (pl. xıx, fig. 3 a) est à peu près plate, ou du moins on ne voit qu'une faible dépression sub-rhomboïdale sur le front où les écailles sont un peu plus grandes que sur le museau : ces écailles ne sont pas carénées. La scutelle occipitale est grande, irrégulièrement campanuliforme; par son bord antérieur, qui est le plus large, elle est en contact avec une petite plaque inpaire et avec les demi-cercles squameux des régions sus-orbitaires, lesquels se touchent, sur le vertex, par leur convexité. Sur les còtés de l'occipitale, il y a des plaques assez grandes; les postérieures, beaucoup plus petites, sont bordées, à droite et à gauche, par des crêtes peu saillantes qui, du bord postérieur de chaque orbite, se dirigent vers l'occiput où elles se réunissent pour former un triangle ouvert en avant, et dont le sommet est l'origine du petit pli cutané de la ligne médiane du dos. Sur chaque région sus-oculaire, on voit un disque de ro à 12 scutelles plates entouré par de fines granulations. - Toutes les pièces de l'écaillure sont généralement petites. - La queue, assez forte et un peu déprimée à sa base, est environ une fois et demie aussi longue que le tronc et la tête; elle est trèseffilée. - Le fanon est petit.

La teinte générale, qui semble être un gris violacé, est un vert clair, comme le montre un dessin fait d'après le vivant, par M. le comte de Castelnau. Sur la tête, il y a de nombreuses vermiculations brunes. Les épaules sont couvertes par une grande tache de la même nuance, qui se prolonge en une pointe sur le cou et s'étend en arrière et en bas jusque sur les bras. 'Trois autres taches, larges de $0^{\mathrm{m}}, 10$ au milieu, et irrégulièrewent angulaires comme la précédente, se voient sur le tronc; leurs angles postérieurs descendent obliquement d'avant en arrière, le long des flancs, et se rejoignent presque sur le ventre, dont la couleur est un peu plus claire 
que celle des régions supérieures. - La mâchoire inférieure, tout à fait en avant, porte une petite tache transversale brune; une autre, plus large, occupe tout l'espace qui sépare le bord inférieur d'un orbite du bord correspondant de l'autre orbite; une troisième, passant sur le fanon, s'étend d'un tympan à l'autre. Au delà, en avant des épaules, une dernière tache transversale forme un collier. A la queue, il y a des anneaux bruns; les membres ne sont que demi-annelés, leur face interne ne portant aucune tache.

L'échantillon unique de cette espèce a été rapporté du Brésil par MM. de Castelnau et Émile Deville.

Par l'ensemble de ses caractères, cet Anolis se rapproche surtout de l'espèce de l'Amér. septentr. nommée, par mon père et par Bibron, An. alligator. La différence de patrie et les détails dans lesquels je viens d'entrer suffisent pour montrer les différences caractéristiques.

XXII. - 22 bis. Arolis hétÉRoderme. Anolis heterodermus, A. Dum.

$$
\text { Pl. xIx, fig。 } 4,4 a \text { et } 4 b \text {. }
$$

Idem, Id., Cat. Rept.du Mus. Paris, p. 599.-Id., Répert. Erpét.génér., D. B., t. IX, p. 262.

Régions supirieure et latérales du tronc recouverles de squames irrégulièrement polygonales, aplaties, lisses, entremclées d'écailles beancoup plus petites et comme granuleuses; plateau crânien bordé dans tout son pourtour, ainsi que le museau, de grandes ́́cailles bombres; squames ventrales lisses, non granuleuses; une petite carène dentelée sur le cou; le dos et la queue.

La tête est un peu effilée ( $\mathrm{pl}$. xıx, fig. $4 a$ ). La faible dépression de sa face supérieure semble ètre plus considérable qu'elle ne l'est réellement, à cause de Ja saillie prononcée des écailles qui bordent l'occiput, les régions susoculaires et le museau. La plaque occipitale irrégulièrement polygonale ou presque circulaire est entourée de plaques de forme et de grandeur variables.

Le fanon est très-peu développé. - La queue, médiocrement longue, est assez robuste et revêtue, dans toute sa longueur, d'écailles à carène saillante.

Le caractère le plus remarquable est foumi par l'aspect singulier de l'écaillure du dos et des flancs; elle offre une assez frappante analogie avec celle de l'An. caméléonide, décrit et figuré par Cocteau (Erpét. in Hist. de Cuba, par Ram. de la Sagra, p. 145, pl. xv), sous les noms re Chamœeleolis Fernandina. Ce sont des écailles plates, entremêlées de petits grains squameux (voy. notre pl. xix, fig. $4 b$ ). Outre d'autres particularités inutiles à rappeler ici, la disposition réguliere des plaques de l'abdomen éloigne com- 
plétement l'An. hétéroderme du précédent, qui est le seul dans ce vaste genre où les écailles ventrales soient granuleuses an lieu d'être, comme d'ordinaire, des scutelles plates et imbriquées.

Les parties supérieures sont vertes et les inférieures, plus claires, ont une teinte jaune verdâtre. Les grosses écailles qui bordent la tête comme une sorte de couronne sont d'un vert très-pâle, de sorte qu'elles paraissent presque blanches; il en est de mème pour celles qui se voient sur les còtés de la tète et de la région cervicale, d'où elles gagnent les flancs en formant sur ces diverses régions une bande blanchâtre, qui tranche sur la teinte générale. - I.ong. totale, $\mathrm{o}^{\mathrm{n}} 17 ; \mathrm{O}^{\mathrm{m}} 6$ pour la tête et le tronc; $\mathrm{o}^{\mathrm{m}}$ I 1 pour la queue. - Nous possédons plusieurs échantillons de cette espèce reçus de la Nouvelle-Grenade.

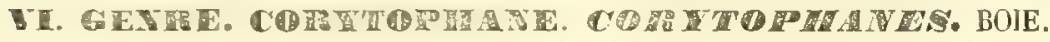

A l'époque où le t. IV de l'Eréél. génér. fut publié, les Corytophanes manquaient au Musée de Paris, où l'on connaît maintenant le Cor. à crête par trois beaux exemplaires provenant de la province de Peten (Amér. centrale) et dus à la générosité de MI. Arthur Morelet, qui a exécuté, d'après le vivant, un dessin propre à bien faire connaître le système de coloration. On acquiert ainsi la preuve que la teinte générale du tronc est un mélange de blanc pur et de brun rougeâtre moins abondant sur les flancs que sur le dos, où il forme de fines bandes transversales. Le blanc est sans mélange sur le ventre. La gorge et le capuchon sont d'un vert clair uniforme. Sur la queue et sur les membres, qui sont de la même couleur verte, il y a des anneaux d'un brun violacé. Des lignes noires, partant de l'orbite, rayonnent en avant, en bas et en arrière.

11 y a, dans les écailles du tronc, plus d'inégalité qu'il n'est dit dans les descriptions, car les flancs sont parcourus de hant en bas par des rangées verticales et irrégulières d'écailles plus grandes que les autres et rappelant un peu les bandes bien moins nombreuses du Cor. caméléopside, chez lequel, d'ailleurs, ces écailles, beaucoup plus grandes, portent une forte saillie médiane (voy. Herpet. mex., p. 38 et pl. vi), tandis qu'elles sont planes ou à peine carénées dans le Cor. à crête. La tête et la partie antérieure du tronc Archives du Muséum. T. VIII. 
de ce dernier sont représentées de profil sur notre pl. xx, fig. I; région suscéphalique, fig. I $a$; tête déponillée de ses parties molles, fig. I $b^{1}$.

XXIII. - I bis. Corytophane trìs-Caréné. Corytoph. percarinatus, A. Dum. espece NoUvelle. - Pl. xx, fig. 3 et $3 a$.

Une crête non interrompue depuis l'occiput jusqu'à la queue; écailles presque toutes égales entre elles et toutes carénées; deux plis cutanés de chaque cóté du tronc commençant à la ré̉jiun cervicale, et prolongés jusqu'aux membres postérieurs.

La face supérieure de la tête, inclinée en avant, est prolongée à sa partie postérieure par une crête osseuse formant une sorte de casque très-analogue à celui des deux autres espèces de ce genre, et qui rappelle la structure bizarre du crâne des Caméléons. Il résulte de cette conformażion une surface rhomboïdale presque plane sur le museau et sur le front, mais un peu creuse au delà des orbites. Elle a des limites saillantes : ce sont, en avant, les crêtes surciliaires, puis au delà, les bords d'une surface triangulaire formée par deux lames osseuses qui, partant de l'occiput et se réunissant au-dessus et en arrière, constituent les deux racines supérieures et antérieures du casque, dont la troisième racine naît d'un point plus inférieur de la région occipitale. C'est de la réunion de ces troís racines ainsi disposées en pyramide triangulaire que provient le prolongement lamelliforme du casque. Telle doit être éviảemment la structure de cette région postérieure du crâne de l'espéce dont il s'agit, à en juger par l'analogie remarquable que présente la tête revêtue de ses téguments avec celle du Corytophane à créte, laquelle est représentée dépouillée de ses parties molles sur notre pl. xx, fig. I b. L’angle antérieur de la surface rhomboïdale sus-céphaliqũe et les angles latéraux situés de chaque côté dans le point où cesse la crête surciliaire et où commence, en lui faisant suite, mais en se portant un peu en dedans, la racine du casque, sont bien moins aigus que l'angle postérieur. Tout le pourtour de cette surface est garni de grandes écailles droites à

1. Le Muséum ne possedde pas le Cor. caméléopside; c'est par erreur que le contraire est dil dans le Catal. des.Rept. du Mus. de Paris, p. 60. - Afin de montrer les différences qui distinguent les deux espèces anciennement connues et celle qui est décriłe, sous le $n^{\circ}$ xxur, 1 bis, j’ai fait reproduire sur cette mème pl. xx, fig. 2 un décalque du dessin donné par M. Gravenhorst (Nova acta nat curios., t. XVI, pars poster. 1833, pl. Lxv, fig. 1). Une représentation peu soignée de ce mème Saurien se trouve in The zool. of Captain Beechey's, voy. 1839, pl. xxx, fig. 1 ; et il y est décrit p. 94, par M. Gray, qui, à l’exemple de Wiegmann, le nomme Chamaleopsis Hernandesii. 
casene trauchante, qui forment une arête vive; elles en élèvent les bords et par cela mème la font paraître, surtout en arrière, plus creuse eneore qu'elle ne l'est en réalité.

La peau qui recouvre le casque se continue avec celle du dos en formant nu repli, véritable crête nuchale finement dentelée, dont la crête dorsale, à dentelures plus prononcées, est la continuation; cette dernière devient de moins en moins haute en se prolongeant vers la queue, où elle est remplacée par une ligne saillante, qui résulte de l'arrangement régulier des écailles médianes surmontées d'une carène, comme le sont d'ailleurs toutes les autres écailles de cette région, garnie ainsi de stries parallèles. La même régularité, au reste, se remarque dans l'arrangement des écailles des membres et du ventre; elles sont plus grandes que celles des autres parties du corps, et chacune d'elles étant carénée, de nombreuses stries parcourent. en long l'abdomen et les pattes jusqu'à l'extrémité des doigts, dont Ies écailles inférieures, particulièrement aux membres pelviens, semblent un peu tuberculeuses. Sur la crête nuchale, sur le dos et sur les flancs, l'écaillure est beaucoup moins régulière, car les pièces squameuses qui la composent ne forment pas des rangées soit longitudinales, soit transversales; quelques-unes, cà et là, paraissent plus grandes que les autres, mais il n'y a pas, sous ce rapport, les différences tranchées si caractéristiques dans les ceux antres espèces de ce genre où les écailles de dimensions plus considérables, comme on le voit surtout dans le Coryloph. caméléopside, forment des bandes transversales. Il faut ajouter que toutes les écailles sont carénées, ce qui est une note distinctive très-importante. Celles de la gorge et du fanon, plus saillantes que les autres, sont tuberculeuses et entourées de fines granulations. La lèvre supérieure porte 2 I plaques, et l'inférieure en al 17 ; les unes et les autres sont suivies de petites plaques tuberculeuses disposées sur deux rangs qui s'étendent jusqu’à l'oreille. - L'orifice de la narine, percé dans une seule plaque, est dirigé d'arrière en avant. - Les panpières sont couvertes d'un nombre considérable de fines granulations. Sur la région sus-céphalique, il y a des écailles carénées et plus ou moins surmontées de petites élévations qui leur donnent un aspect rugueux. Enfin, on ne voit pas au-dessus du tympan, dont la membrane est tout à fait superficielle, la saillie qui manque également dans le Cor. à crête, mais que, dans le Cor. caméléopside, Wiegmann a signalée comme une proémi- 
nence de l'os temporal en forme d'aiguillon. Le fanon, contrairement à ce qui a lieu đans cette dernière espèce, est dentelé à son bord inférieur, qui se termine en arrière par un repli cutané transversal, continué sur la partie la plus inférieure de chaque flanc, jusqu'à la racine des inembres abdominaux. Un autre pli, semblable au précédent, commence derrière l'angle de la mâchoire, décrit d'abord une légère courbe à concavité inférieure; puis au niveau des épanles, il se dirige parallèlement à la ligne médiane du dos, dont il est peu éloigné, et vient se perdre sur l'orígine de la queue.

La teinte générale est un brun verdâtre. La face supérieure de la tête est ornée en avant, à sa partie la plus antérieure, d'une tache noire placée entre deux lignes également noires qui, partant du milieu de la région sus-orbitaire, vont en divergeant se porter sur le bord du musean et descendent au-devant de l'œil sur la région frénale; deux autres lignes semblables, mais dirigées en sens absolument inverse, prennent naissance dans le même point et vont atteindre, en s'écartant, les bords du casque, les franchissent et se perdent sur la crête nuchale. De ce point central inter-orbitaire, d'autres raies noires, disposées en éventail, couvrent de dedans en dehors toute la portion un peu renflée qui correspond à l'oeil et contournent la crête surciliaire pour gagner la paupière supérieure. Une tache sombre transversale occupe l'extrémité de la crête de la nuque et s'arrête, de chaque côté, au niveau du pli cutané supérieur. D’autres maculatures noires irrégulières sont dispersées sur différents points du tronc et de la queue.

Long. totale, $\mathrm{o}^{\mathrm{mn}} 285$; tête et tronc, $\mathrm{O}^{\mathrm{mm}} 75$; queue, $\mathrm{o}^{\mathrm{mn}}$ 2 Io; espace compris entre le bout du museau et l'extrémité supérieure du casque, $\mathrm{o}^{\mathrm{m}} \mathrm{0}$.

Le Cor' très-caréné a été pris à Ascuintla, dans l'Amér. centrale, à 3o lieues de Guatemala. Le spécimen est unique.

La description qui précède montre, sans qu'il soit nécessaire d'y insister, les différences tranchées qui distinguent ce Corytophane de ses congénères. Ainsi, pour les rappeler en peu de mots, s'il porte une crête nuchale comme le Cor. à crête, remarquable par son écaillure du dos et des flancs presque partout lisse, il a, au contraire, toutes les écailles carénées et sensiblement égales entre elles. Ce défaut d'inégalité est un caractère important quand on compare l'espèce nouvelle au Cor. caméléopside. Chez ce dernier d'ailleurs, $1^{\circ}$ la crête commence seulement au niveau des épaules; $2^{\circ}$ les grandes écailles disposées en bandes transversales sont seules carénées, et $3^{\circ}$ enfin, chaque 
flanc ne porte que deux petits plis cutanés : l'un à la région scapulaire et l'autre à la région pelvienne.

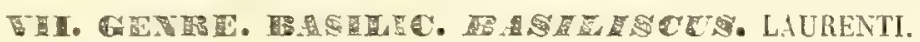

Sous le nom générique proposé d'abord par Laurenti pour le Saurien que Linné avait nommé Lacerta basiliscus, les auteurs de l'Erpét. génér. ont réuni les deux espèces alors connues, celle qui est le type du genre ( $b$. mitratus) et celle que M. Kaup a désignée sous la dénomination de Cor) theolus vittatus, dont on doit rapprocher le genre nominal OEdicoryphus (vittatus) que Wagler a introduit dans son Syst. amphib., p. 148. - Ce groupe, jusqu'alors très-circonscrit, a été angmenté par M. Gray d'un genre à tête ronde et simple en arrière, ou du moins à très-petite crête occipitale, mais à bord externe des doigts postérieurs garni d'une frange. Il en a décrit le type (Beechey's, voy. p. 94) sous le nom: Ophyessa bi-lineata. Un exemplaire de cette espèce, donné par le Musée de Londres, a été considéré avec raison par mon père et par Bibr., t. Iv, p. r88, comme un jeune $B . \dot{a}$ bandes. Ce genre a été conservé par M. Gray dans son Cat. of Liz., p. 193, mais sous un titre différent, car on l'y trouve ainsi désigné : Thysanodactylus bilineatus de l'Amér. du Sud et de l'île de Fernando-Noroha. Je dois, au reste, appeler l'attention sur cette origine, puisque le $B$. ̀े bandes est mexicain ou provient de l'Amér. centrale. - Tout récemment, ainsi que je l'ai indiqué dans la liste dressée d'après le système cle classification de M. Gray (p. 509), ce zoologiste a présenté nne nouvelle distribution du groupe des Basilics (Basiliscina), motivée par l'adjonction de genres jusqu'alors inconnus (Ann. of nat. Hist., $2^{\circ}$ série, 1852, t. x, p. 437). Les différents Sauriens qui y sont compris manquent dans nos collections. Ils sont originaires, les uns du Mexique, et les autres de l'Amérique du Sud ${ }^{1}$.

1. W. Gray forme des genres suivants une sectiun : Basiliscina, partagée en 3 groupes: I. Occiput renflé de chaque côté avec une haute crète cutanće comprimée et partant du bord postérieur des yeux : 1. Ptenosaura Gr. (Pt. Seemanni Gr). - 11. Occiput renflé couvert d'écailles convexes; à sa région postérieure, mais à une certaine distance en arrière des yeux, il est prolongé en une haute crète cutanée comprimée; sur le dos et sur la queue, une crête élevée supportée par des rayons osseux: 2. Basiliscus, Laur. (B. americanus, Id.); 3. Lophosaura, Gr. (L. Goodridgii, Id.); 4. Cristasaura, Gr. (C. mitrella, Id.); ร. Corytheolus, Kaup (C. vittatus, Id.). - III. Occiput aplati, avec une trèspetite crèle comprimée sur le milieu de son bord postérieur : 6. Thysanodactylus, Gr. (T. bilineatus, Id.). 
Avant de présenter la description de la nouvelle espèce de notre Musée, je ferai observer que si la crête occipitale des Corytophanes diffère beaucoup de celle des Basilics, en ce qu'elle a pour base la longue apophyse osseuse qui prolonge le crâne en arrière (pl. xx, fig. I $b$ ), tandis que dans les autres, la crête est essentiellement formée par la peau relevée en pli mince et d'une étendue plus ou moins considérable, selon les espèces, le prolongement osseux ne manque cependant pas complétement aux Basilics. Il est bien moins saillant, il est vrai ( $\mathrm{pl} . \mathrm{xx}, \mathrm{fg} \cdot 4$ ), car il se présente sous la forme d'une lamelle mince horizontale, à bord supérieur tranchant ế qui reste dans le plan de la région sus-céphalique, au lieu de se relever obliquement, comme le grand casque des Corytophanes, dont la base, ainsi que je l'ai déjà rappelé plus haut, est constituée par trois racines aplaties et disposées en pyramide triangulaire terminée à son sommet par une lame mince et transparente. Cette conformation remarquable manque dans les Basilics (fig. 4), où la lamelle, mesurée à son bord inférieur, est à peine égale au tiers de la longueur du crâne prise du milieu de l'arcade maxillaire supérieure à l'articulation de l'occipital latéral avec l'os intraarticulaire. Dans le Corytophane dont la tête a été dessinée, il y a, au contraire, égalité entre ces dimensions. - Sur ceite même pl. $\mathrm{xx}$, on voit que les dents, dans ces deux genres de Sauriens, sont trilobées, puisqu'elles portent une petite dentelure de chaque côté de lenr base (fig. I $c$ et $4 a$ ).

XXIV. - I bis. Basilic a Bonnet, Basiliscus galeritus, A. Dum.

Pl. XXI, fig. $4,1 a$ et $1 b$.

Idem, Id. Catal. Rept. Mus. Par., p. 64. - Id., Id., Répert. Erpét. gén., D. B., t. IX, P. 264.

Téte surmontée d'un capuchon élevé, large et épais à sa base, à sommet mince, arrondi; sur le dos et sur le premier liers de la queue, une crète dentelée peu élevée; écailles des régions supérieures du tronc carénées, les ventrales lisses; celles des régions sus-oculaìres petites, à surface rugueuse; parties supérieures vertes, et les inférieures d'un jaune verdatre; sur la queue, des taches brunes.

La tête est courte et le capuchon ne ressemble pas à un bonnet phrygien, comme celui du $B$. ̀̀ capuchon (pl. xxı, fig. 2), et il n’est pas pointu comme celui du $B$. àbandes (pl.xxi, fig. 3). Chez le mâle, comme chez la femelle, il commence au niveau du bord postérieur de l'espace inter-orbitaire, et chez le premier, son sommet, très-régulièrement arrondi, décrit un arc de 
cercle uni à la base, antérieurement, par un bord très-court, un peu oblique d'avant en arrière, et postérieurement, par un bord plus long rectiligne, et dont l'obliquité est en sens inverse. Chez la femelle, le capuchon est beaucoup moins vertical; il se dirige en arrière, et son sommet représente assez exactement l'une des extrémités d'une ellipse. Dans les deux sexes, d'ailleurs, il rappelle un peu, par sa forme, le capuchon du camail ou habillement d'hiver que les ecclésiastiques portent par-dessus le rochet. Ce prolongement cutané, qui est très-mince, est couvert d'écailles assez grandes presque planes; mais sur la base renflée du capuchon, sur les tempes et à la région sus-céphalique, elles sont moins volumineuses et ressemblent à de petits tubercules.

Par l'ensemble de ses formes, ce Basilic offre la plus grande analogie avec ses deux congénères. - Je viens d'indiquer la différence importante résultant de la conformation toute particulière du capuchon; il est donc inutile, surtout en mentionnant l'absence de la remarquable crête, à rayons osseux du dos et de la queue, d'insister sur les antres caractères qui l'éloignent de l'espèce la plus anciennement connue et que Daudin a, le premier, nommée $B$. mitratus. - Avec le $B$. à bandes Wiegm., qui porte une carène sur les écailles ventrales, aucune confusion n'est possible, puisque, chez les deux antres, cette carène manque.

Le système de coloration est fort simple, comme on le voit sur la fig. I, où les teintes sont plus vives que sur les animaux conservés dans l'alcool. - Elle représente le mâle de grandeur naturelle; il est un peu plus petit que la femelle. - Le Muséun possède deux individus de sexe différent; ils ont été rapportés de la Nouvelle-Grenade.

- Un dessin, de M. Morelet d'après l'un des exemplaires adultes du Basilic à bandes recueillis par lui dans la province de Peten (Amér. centrale), fait bien connaitre le système de coloration de ces beaux Sauriens déposés par ce voyageur dans nos collections; mais ils sont malheureusement déjà un peu décolorés par l'action de la liqueur. 11 en est de même pour un jeune sujet, également donné par M. Morelet. - Au moment où l'un des animaux adultes a été pris, il était d'un vert clair vermiculé de noir; on voit encore les courtes bandes transversales noires caractéristiques sur la nuque et sur le dos, et en partie une raie jaune étendue de l'angle postérieur de l'œil jusqu'à la cuisse; puis au-dessous et parallèlement, sur le cou, une autre 
ligne semblable à la précédente. La tête et le capuchon étaient et sont encore bruns, mais on ne retrouve plus que sur le dessin cette dernière indication : lèvres et gorge d'un blanc pur. Chez le jeune individu, il n'y a sur la tête que de faibles vestiges du capuchon qui manque dans les premiers temps de la vie, comme on en acquiert la preuve par l'examen d'un trèsjeune sujet provenant du Mexique. Il en est de même pour trois jeunes $B$. à capuchon originaires, soit de Cuba et donnés par M. Ramon de la Sagra, soit du Mexique; l'un de ces demiers est un présent de M. Castelnau. Entre ces jeunes animaux et ceux de même âge qui appartiennent à l'espéce dite $B$. à bandes, il y a une très-grande analogie pour la disposition et l'aspect, non-seulement de la double bande claire latérale, mais de la petite raie jaune médiane de la région sus-céphalique. Il est cependant très-facile de les distinguer en examinant les écailles ventrales, qui sont lisses chez le $B . \grave{a}$ capuchon, et carénées au contraire chez le $B$. à bandes. - Un Bas. appartenant à cette dernière espèce, et tout récemment acquis par notre Musée, doit être signalé, parce qu'il présente une particularité notable qui n'avait pas encore été constatée dans cette espèce, car il a sur le dos une crête bien développée, soutenue par des rayons osseux et semblable à celle du $B$. is capuchon. Sur la queue, il n'y a qu'une carène dentelée. Il est plus petit qu'un autre individu de la même espèce à crête dorsale, cependant moins haute et sans rayons osseux.

Le genre Amblyrhy nete, dont je dois maintenant parler, est précédé dans la classification de l'Erpét. génér. du singulier genre Aloponote (8), caractérisé par l'absence d'écailles sur les parties supérieures du tronc, où l'on ne voit que de très-petits grains squameux serrés et fort nombreux. Je n'ai rien à ajouter à l'histoire de ce Saurien, connu au Musée de Paris par un seul individu de grande taille, envoyé de Haïti par M. Alex. Ricord, et dont aucun Musée ne paraît avoir reẹ de nouveaux exemplaires.

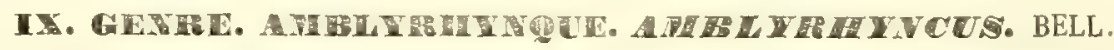

La diagnose de ce genre a été donnée dans l'Erpét. génér. d’après un spécimen en mauvais état de conservation de l'Ambl. à créte appartenant à M. Th. Bell, et d'après un exemplaire d'une autre espèce du Musée de Boulogne-sur-Mer dédiée par mon père et par Bibron au directeur de ce Musée : Ambl. de Demarle. Or, l'examen de nouveaux sujets conservés 
dans l'alcool et recueillis aux îles Galapagos, par M. Darwin, a démontré à M. Bell que, si la description de la seconde espèce ne laisse rien à désirer, il n'en était pas de même pour la première; car celle-ci offre des particularités remarquables qui n'avaient pu être signalées ni par M. Bell en I 825 (Zool.Journ., p. 204, pl. xu supplém.), ni en 1837 , par les auteurs de l'Erpét. génér. Il faut donc modifier l'énoncé des caractères génériques pour ce qui concerne la queue et les membres, et remplacer ces deux phrases: queue comprimée vers son extrémité et garnie de grandes écailles; doigts gros et courts, par les indications suivantes: queue ronde ou comprịmée, doigts assez longs et inégaux et complétement libres, ou presque égaux et un peu palmés, Ces différences importantes sont liées à celles qui se remarquent dans le genre de vie; elles sont telles que l'A mbl. de Demarle, dont la queue est cylindrique et dont les doigts ne sont pas réunis par une membrane, est un animal essentiellement terrestre et que l'Ambl. à crête, si distinct du précédent par sa queue comprimée d'un bout à l'autre et par la palmure partielle des doigts aux pattes antérieures et postérieures, est une espèce tout à fait aquatique. On doit, en outre, noter que la crête de celui-ci est très-basse au-clessus des épaules, où elle semble comme interrompue, et que chez l'Ambl. de Demarle, elle a plus d'élévation sur le cou que sur le dos, sans présenter cette sorte d'interruption que je viens de signaler ${ }^{\text {. }}$

1. M. Ch. Damin a donné de longs détails très-précis sur ces Reptiles, qu'il a vus en grand nombre cus illes Galapagns (Journ. and remarks, Voy. of the Beagle, p. 466-472, 1839, et a' édit. $185 \%$, p. 38\%-390, arec une fig. de l'Ambl. à crete). Les individus appartenant à cetto dernière cspèce habitent exclusivement, dit-il, les rochers du rivage qu'ils paraissent ne jamais quitter pour pénétrer dans les terres, et sur lesquels ils s'empressent de revenir, dès qu'ils ont été chercher dans la mer leur nourrilure, qui ne se compose que de plantes, et particulièrement de celles dont la végétation a lieu au fond des eaux. Ce naturaliste s'en est assuré en ouvrant plusieurs de ces Ambl., et jamais il n'a trouvé dans leur estomac des débris de poissons ou d'autres animaux marins. Ils ont une teinte noiràtre, uniforme, et leur taille peut dépasser un mètre.

Quant à l'A mbl. de Demarle, il n'est pas répandu comme l'autre, sur toutes les lles Galapagos; il esí confiné dans celles qui forment le centre de l'archipel. Quelques-uns de ces Sauriens habitent les régions hautes et humides des îles, mais ils sont beaucoup plus nombreux dans les parties basses et stériles, non loin des côtes; on les y rencontre en telle quantité, qu’à l'ŝle St.James, les voyageurs ne purent trouver pour dresser leur tente un emplacement non occupé par les habitations souterraines, et d'ailleurs peu profondes, de ces reptiles. Comme les Ambl. maritimes, ce sont des animaux assez laids, à physionomie stupide, en raison du peu d'ouverture de leur angle facial (d'où le nom d'Amblyrhynque ou á museau obtus); ils sont, en dessous, d'un rouge orangé tirant sur le jaune, et en dessus, d'un brun rougeâtre. Leurs mouvements sont lents, et ils se traînent sur le sol plutôt qu'ils ne marARChIVES DU HUSÉuM. T. VIII. 
- Je n'ai rien à ajouter aux détails donnés par mon père et par Bibron sur les denx genres Iguane ( IO) et Mḱtopocḱros ( $\mathrm{I}$ ). Je dirai seulement qu'un $1 g$. tuberculeux a pu être observé vivant à la ménagerie où ses habitudes d'animal essentiellement frugivore ont subi une singulière modification; car on a pu le nourrir pendant trois mois avec de jeunes moineaux pris au moment de l'éclosion de l'œuf, et de larves de Ténébrions qu'il mangeait avec beaucoup plus d'avidité que les fruits, dont il avait jusqu'alors fait exclusivement usage pendant une captivité d'une année entière.

Quant au genre Crclube (12), des observations intéressantes ont également pu être faites sur le régíme d'un individu appartenant à l'espèce dite C. de Harlan Wiegm. (C. carinata Harl.). Malgré son genre de vie habituel, ce grand Saurien qui, à l'état de liberté, ne recherche comme aliments que des fruits ou d'autres productions végétales, mange volontiers maintenant des larves de Ténébrions, de très-jeunes souris et de petits oiseaux qui viennent de sortir de leur coquille.

Le Cycl. pectiné, décrit par Wiegmann d'après un spécimen unique du Musée de Berlin et inconnu à Londres, ainsi que dans les différentes collections d'Allemagne, comme on le voit d'après les indications fournies en I 845 par M. Fitzinger, vient d'être acquis tout récemment par le Musée de Paris. Il est parfaitement semblable à l'animal figuré dans I'Erpét. du Mexique, pl. Ir, et très-distinct du Cycl. de Harlan, qui a, comme lui, la queue comprimée, $1^{\circ}$ par la continuité de la crête, qui est sans interruption audessus des épaules, mais en présente une à la région lombaire, et $2^{\circ}$ par le petit nombre des pores fémoraux, dont on compte non pas vingt, mais cinq seulement à chaque cuisse. Aucune confusion d'ailleurs ne peut exister entre cette espèce rare et le Cycl. acanthure, dont la queue, plus

chent. Ils se nourrissent de matières végétales, et dans les lieux secs où ils ne peuvent trouver de I'eau ils recherchent avec avidité les branches de cactis ou d'autres plantes pourvues d'un suc abondant. Leur chair, après la cuisson, est blanche et d"une saveur agréable pour ceux, dit M. Darwin, qui, relativement au choix de leurs aliments, savent se mettre au-dessus des préjugés. Leurs œufs sont également estimés.

Je n'ai pas craint de présenter les détails qui précèdent, parce qu'il est fort intéressant de bien connaître un genre aussi nettement caractérisé, comprenant une espèce marine et une espèce terrestre, et propre à une région du monde très-délimitée comme l'est l'archipel des Galapagos. De plus, l'Ambl. à crête est extrèmement remarquable en ce qu'il est le seul Saurien connu dont la nourriture so compose de plantes qui croissent dans la mer. 
effilée, est presque ronde, et dont la crête n'est nullement interrompue ${ }^{4}$.

Le genre Brachylophe (13) ne renferme encore que l'espèce nommée par Alex. Brongniart Iguane à bandes, et qui a servi de type à Cuvier pour cette coupe générique. Ce Saurien est le seul, dans la sous-famille des Pleurodontes, qui ne vive pas en Amérique. C'est du moins ce qui semble résulter de l'examen de nos échantillons, dont les uns, cités dans l'Erpét. génér., ont été recueillis à Tongatabou (Archipel des Amis ou de Tonga, Océanie), et dont les autres, reçus depuis 1837 , ont été rapportés par M. Leguillon, ainsi que par M. Arnoux, de différents points de l'Océanie, et en particulier de l'île Wallis (Archipel Ona-Hourn). Au Musée de Vienne, selon les indications données par M. Fitzinger, qui nomme cet Iguanien Hypsilophus fascialus, en adoptant le mot Brachylophus comme simple dénomination de sous-genre (Syst., p. 55), on ne possède que des exemplaires de l'AncienMonde. Dans le Musée britannique: au contraire, M. Gray signale seulement des individus de l'Amér. du Sud.

On constate dans notre Musée que les femelles, caractérisées par la présence, sur la face interne de chaque cuisse, de huit ou neuf écailles suborales, munies d'une petite fente près de leur bord postérieur, portent seules des bandes transversales bleues sur le dos et de gros points de la mème couleur sur le cou et les épaules. - Les mâles, qui sont munis de Téritables pores aux cuisses, ont une teinte bleuâtre obscure et uniforıne, sans bandes; les points bleus de la région cervicale sont à peine apparents.

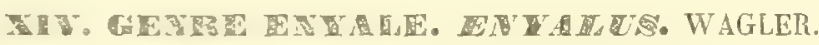

Tine rectification doit être faite dans l'Erpét. génér. à l'énoncé des caractères des Reptiles de cette division, car c'est par erreur, ainsi qu'il est facile de s'en assurer sur les individus mêmes qui ont servi aux descriptions de ce livre, que l'En. rhombifére y est indiqué comme ayant les écailles du

1. Quelques espèces nouvelles du genre Cyclure ont été décrites dans ces dernières années; leurs noms se trouvent dans les listes que j'ai donncées ( p. 509) d"après le Cat. de M. Gray et p. ä06 d'après le Syst. de M. Fitzinger. Nolre Musée ne les possède pas. On n'y connaît pas non plus une espèce de la Jamaĩuve, dite Cyclura lophoma (2.000\%, crète, w.rs, épaule) Gosse (Ann. of nat. hist., 2o série, 1849 , t. IV, p. 64-68). Aux détails zoologiques, ce naturaliste a joint un extrait intéressant des observations de M. R. Hill sur les mcurśs de ce Saurien, dont l'alimentalion est exclusivement végétale. 
ventre lisses. Les carènes de cette région sont moins prononcées que chez l'En. à deux raies; néanmoins, on voit sur chaque pièce de l'écaillure abdominale une ligne peu saillante, il est vrai, mais l'ensemble de ces carènes constitue des séries de stries longitudinales sur toute la face inférieure du tronc. Ce qui distingue surtout les deux espèces, c'est, chez l'En. à deux raies, $1^{\circ}$ la présence sur la ligne médiane du dos de plusieurs rangs longitudinaux de grandes écailles et non pas d'une seule rangée comme chez l'En. rhomb.; $2^{\circ}$ le nombre moindre et le volume plus considérable des écailles sus-orbitaires. I'En. à deux raies, dont on possédait un seul spécimen à l'époque où il fut décrit, est maintenant bien connu au Musée de Paris, où l'on en a reçu trois nouveaux exemplaires du Brésil et parfaitement identiques au type. En outre, un jeune individu de cette espèce, assez décoloré, mais très-reconnaissable, a été rapporté de ce pays, ainsi que plusieurs En. rhombif. La teinte générale de ces derniers est un brun uniforme, si ce n'est chez un seul, qui est exactement semblable par ses taches dorsales, ovalaires ou irrégulièrement rhomboïdales, à l'animal dessiné par Spix, pl. xr et type de son Lophyrus rhomb. Ses écailles ventrales sont carénées ${ }^{1}$.

- D'autres Enyales, recueillis à Fonteboa (Haut-Amazone, partie brésilienne) par MM. de Castelnau et Em. Deville, n'appartiennent ni à l'une ni à l'autre espèce dont je viens de parler. C'est de l'En. rhomb. qu'ils se rapprochent le plus; mais l'aspect rugueux de leur tête, puis l'élévation plus considérable de la crête dorsale et sa prolongation sur la base de la queure leur donnent une certaine ressemblance avec le Lophyrus margarilaceus, Spix (Lacerke brasil., p. 10, pl. xır, fig. 1), connu dans les Musées de Munich, de Vienne et de Berlin (Fitz., Syst., p. 58) et rapporté, mais avec donie, à l'En. rhomb. dans l'Erpét. génér. Le dessin de l'ourvage de Spix est si insuffisant et la description est si peu explicite, qu'il est bien difficile, au reste, de ne pas se borner à de simples conjectures sur l'identité du Loph. marga. rit. et des Sauriens dont M. de Castelnau a fait présent. Quoi qu'il en soit,

1. D’après la rectífication à faire à la diagnose donnée pour ce genre dans l'Erpét. gén, il ne peut plus maintenant rester de doutes sur l'erreur qui, dans nos collections, avait fait rapporter à l'En. à deux raies tous les individus munis de ces carenes; il faut donc nécessairement laisser de còté le observations consignées dans le Cat. du Musée de Paris à l'occasion du spécimen dont il s'agit, et que M. de Castelnau a fait parvenir de Bahia. C'est éridemment un En. vhomb., et il parait incontestable que ce Saurien a été représenté dans le jeune âge par Spix (pl. xur, fig 2) sous le nom de Lophyrus albo-maxillaris. 
ORDRE DES SAURIENS. - IGUANIENS PLEURODONTES.

M. Guichenot a décrit ces derniers comme appartenant à une espèce nouvelle (Explorat. scient.partie centr. Amér. du Sud, Rept., p. 20 et $2 \mathrm{I}, \mathrm{pl}$. v et vi).

XXV. - i bis. Evthale tête-LAR̆ge, Enyalus laticeps, Guich.

Dos et base de la queue surmontés d'une crête à dentelures asser élevées, et dont la hauteur va en diminuant à partir de la nuque; écailles dorsales égales entre elles, petites et un peu pointues; sus-céphaliques et sus-oculaires nombreuses, de petites dimensions et saillantes comme les précédentes, d'où il résulle que toutesles régions supérieures semblent rugueuses; squames ventrales carénées; tête courte et larye.

Par sa conformation générale, cet Enyale ressemble beaucoup à ses congénères; les caractères qui le rapprochent du Rhomb., savoir', $1^{\circ}$ les rangées nombreuses de squames sus-oculaires; $2^{\circ} l^{\prime}$ absence de plusieurs rangées de grandes écailles carénées sur le dos, et $3^{\circ}$ le peu de saillie des carènes des squames ventrales, l'éloignent de l'En. à deux raies. Ce qui le distingue de la première de ces deux espèces, c'est l'aspect plus rugueux de toute l'écaillure, les dimensions plus considérables des grandes écailles pointues de la ligne médiane du dos, et dont l'ensemble constitue une crête un peu épineuse et plus proéminente que dans le Rhombifère, surtont à la nuque, où elle est plus haute ique partout ailleurs, puis qui se continue avec l'apparence d'une car'ene saillante sur la base de la queue; c'est, en outre, la grandeur et la forme des pièces de l'écaillure abdominale qui ne sont pas carrées, mais représentent des parallélogrammes plus longs que larges, et parcourus obliquement par une ligne saillante ou carène étendue de l'un des angles supérieurs de la squame à l'angle inférieur opposé. Il faut enfin tenir compte de la conformation de la tête, qui est un peu plus courte et plus ramassée.

I a teinte générale est verte; sur le tronc, on voit de fines marbrures ou petites taches brunes on noirâtres; on les retrouve sur les membres et sur la queue où elles forment des anneanx ${ }^{1}$.

1. Nous pensons qu'il convient de rapprocher duspécimen qui a servi de type à M. Guichenot pour l'En.tète-large, deux autres individus recueillis dans la mème localité (Fonteboa Haut-A mazone, partie brésilienne) par les mèmes royageurs, et que ce naturaliste, en raison d'une différence dans la conformation de la tète, a considérés comme représentant une espèce distincte qu'il a nommée En. teteplate (loc. cit., p. 21, pl. vi). Ces deux Sauriens, dont la lête est, en effet, un peu plus allongée, mais qui, sous tous les autres rapports, ressemblent au précédent, offrent cette particularité qu'ils portent à la face interne de chaque cuisse trcis pores fémoraux, et que leur gorge est noire. Le mode de préparation de ces animaux ne permet pas de constater leur sexe, mais il y a lieu de penser que ce sont des mâles, tandis que l'autre serait une femelle. 
Les dimensions de deux de ces Enyales sont de $o^{\text {na }} 35$ et de $o^{m} 44 ;$ la queue mesurant, chez l'un, $\mathrm{o}^{\mathrm{m}} 22$, et, chez l'autre, $\mathrm{O}^{\mathrm{m}} 28$.

- A la suite du genre Enyale dont je viens de m'occuper, les auteurs de l'Erpét. génér. en ont placé un autre établi par Boie d'après un Saurien originaire de l'Amérique du Sud, comme les précédents, et nommé par Linné Lacerta superciliosa. C'est l'Ophryesse (I 5), dont la dénomination spécifique rappelle la même idée que le nom de genre tiré par le savant zoologiste hollandais' de la saillie des écailles surciliaires. Si je n'ai pas à m'arrêter sur ce groupe, très-distinct de ceux qui suivent et de ceux qui précèdent, il n'en est pas de même pour un Iguanien reçu du Brésil dans ces dernières années, et qui offre quelque analogie avec l'Ophryesse. Je l'ai fait connaître dans notre Catal., et je dois en donner ici la description.

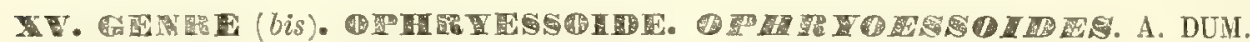

Tête petite, en forme de pyramide quadrangulaire, bordée, de chaque côté par une crête surciliaire; narines latérales; plaque occipitale petite; des dents palatines; toules les pièces de l'écaillure carénées et imbriquées; queue un peu comprimée à sa base, arrondie dans le reste de son étendue et très-efflée à son extrémité, surmontée dans son premier tiers seulement d'une carène dentelée, continue aved celle peu élevée qui règne sur toute la longueur du dos; peau de la gorge sans pli ni longitudinal, ni transversal.

Par tout l'ensemble de sa conformation, le Reptile, type de ce nouveau genre, a de frappants rapports de ressemblance avec l'Ophryesse, c'est ce que j’ai voulu rappeler par la dénomination dont j'ai fait usage. Ces analogies sont : $1^{\circ}$ la brièveté de la tête, couverte d'écailles assez semblables entre elles pour la forme et pour la grandeur; $2^{\circ}$ la petitesse de la plaque occipitale; $3^{\circ}$ la situation des narines sur les côtés du museau; $4^{\circ}$ la similitude de conformation des doigts, qui sont finement dentelés sur les bords, et l'égalité de leurs dimensions respectives, en ce sens que, chez l'un comme chez l'autre, le $4^{\circ}$ est le plus long de tous et le $1^{\text {ex }}$ le plus court; $5^{\circ}$ enfin, la conformité de structure des dents, qui ne sont simples que sur le devant des mâchoires, toutes les autres étant trilobées à leur sommet.

Il y a cependant des différences bien tranchées, qui ont motivé la distinction générique. Ainsi, l'Ophryessö̈de s'éloigne de l'Ophryesse $1^{\circ}$ par les 
dimensions bien moindres de sa queue dont la forme est différente, car au lieu d'ètre comprimée dans toute sa longueur, elle est cylindrique, surtout au delà de sa base; puis, par le peu de hauteur de sa carène dentelée qui, d'ailleurs, ne s'étend pas au delà du premier tiers de la queue; $2^{\circ}$ par l'absence de plis sous la gorge, soit en longueur, soit en travers; $3^{\circ}$ enfin, par le volume proportionnellement plus considérable de toutes les écailles, et en particulier de celles de la tète. - Il n'y a qu'une seule espèce.

XXVT. - I. Ophrxessoïde trois-crêtes, Ophryoess. tri-cristatus, A. Dum.

$$
\text { Pl. xxir, fig. } 1 \text {. }
$$

Idem, A. Dum., Cat. Mus. de Par., p. 66. - Id., Id. Répert. Erp. génér., t. IX, p. 26\%.

Téte courte, épaisse, dont la face supérieure obliquenent dirigée d'arrière en avant et de haut en bas dans la plus grande partie de son étendue, est brusquenent inclinée dans sa portion postérieure, an niveau de la saillie formée, de chaque cóté, par la dernière écaille proéminente du bord surciliaire, et, par suite, région occipilale plus basse et presque perpendiculaire; sur le dos et sur la queue, à droite et à gauche, à une petite distance de la crete médiane, une autre petite crite parallele à celte dernière.

La forme bizarre de la tête de ce Saurien lui donne une physionomie toute particulière. Le corps est un peu comprimé, et les membres sont médiocrement développés; toutes les carènes sont saillantes, mais surtout celles des écailles plus grandes que les autres, qui forment les crêtes médiane et latérales. Parmi les écailles sus-céphaliques, dont aucune n'est dilatée en travers et qui sont toutes carénées, celles des arêtes surciliaires sont les plus saillantes. - Il n'y a point de pores fémoraux chez notre unique individu.

Les parties supérieures sont d'un brun fauve et ornées de bandes transversales sur le dos et verticales sur les flancs, également brunes, mais plus foncées, finement liserées de blanc-jaunâtre. On en voit une sur la tête, entre les yeux, formant un triangle à sommet postérieur fort ouvert et à bord antérieur très-légèrement saillant à sa partie moyenne. Ces taches, bien apparentes sur la queue, y sont très-rapprochées; elles en occupent la région supérieure et les côtés. En dessous, l'animal est d'un brun clair. - Il a été rapporté du Brésil par M. Claussen. - Sa longueur totale est de $0^{\mathrm{m}}{ }_{1} 6$ ainsi répartis : tête et tronc, $o^{m} 06$, queue, $o^{\mathrm{m}} 10$. 


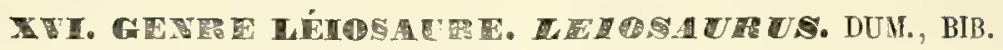

Le Musée de Paris, qui a reçu de l'Acad. de Philad. et de M. le Dr Hallowell, l'un de ses membres, des Rept. intéressants de l'Amér. sept., a obtenu par ce savant naturaliste un très-beau spécimen de l'espèce nommée Agama collaris Say. Ce Saurien non mentionné dans l'Erpét. génér. est devenu pour M. Holbrook le type du genre Crotaphyte qu'il a décrit et figuré d'après un individu vivant ( $N$. Amer. herpet., t. II, 1842, p. 79, pl. x ). Or, quand on étudie comparativement et avec soin les caractères génériques du Crotaphyte et ceux des Léiosaures, on est frappé de leur extrême analogie, car les seules différences qui méritent d'ètre signalées sont que ces derniers manquent de pores fémoraux, et ont la quene médiocrement allongée, taudis qu'elle est longue chez le Crotaphyte, dont chaque cuisse porte une rangée de pores. Si cependant on considère que le genre Léiosaure, vraiment bien distinct de ceux qui lui ressemblent le plus, a été établi par les auteurs de l'Erpét. génér., d'après l'examen d'animaux de petite taille et qui ne sont peut-être pas adultes, il est permis de supposer que leur quene est proportionnellement moins longue qu'elle ne doit l'ètre à une époque plus avancée de la vie. Cette hypothèse, d'ailleurs, est justifiée, et je crois devoir insister sur ce fait, par la comparaison que j’ai pu établir entre deux jeunes Crolaph. et le sujet adulte donné par M. Hallow.; ces individus de petite taille ont la queue courte, et offrent, par conséquent, sous ce rapport, une dissemblance très-marquée avec le spécimen de grande taille. - Quant aux pores des membres, on ne saturait attacher à leur présence ou à leur absence une importance très-grande, puisqu'il n'est pas possible d'affirmer qu'elle n'est pas une manifestation extérieure de la différence de sexes. - Relativement à l'élargissement de la tête au niveau des régions temporales, et que rappelle la dénomination employée par l'habile erpétologiste de Charleston (xpótaøos, tempe), il se remarque également chez les Léiosaures.

Il résulte de ces remarques et de la sinilitude frappante de ces animaux comparés entre eux, que les deux genres dont il s'agit semblent véritablement devoir n'en former qu'un seul auquel le nom de Léiosaure appartiendrait par droit de priorité.

Je n'ai pas de détails particuliers à donner sur l'espèce nommée maintenant dans nos collections Leiosaurus collaris, mais dite d'abord Agama collaris, 
dont la première indication se trouve dans Say (Long's expedit. to rock. mount., t. II, p. 252), puis dans Harlan (Med. and phys. researches, p. I42, pl. sans $\mathrm{n}^{\circ}$ ), et qui enfin, a été l'objet d'une excellente description de la part de M. Holbrook (loc. cit., t. II, p. 79, pl. x). Je dois seulement signaler la présence au Musée de deux jeunes sujets de la même espèce, recueillis à la Nouvelle-Orléans par M. Trécul. Ils offrent une analogie parfaite avec le spécimen adulte, et chez l'un de ces Sauriens, on voit encore les bandes transversales noires du dos indiquées par M. Holbr. comme disparaissant avec l'âge. J'ai déjà parlé de la brièveté relative de leur queue, c'est une différence due à ce qu'ils n'ont pas encore atteint tout leur développement. La tête de l'un de ces Léios. à collier est représentée sur notre pl. xxır, fig. 3. Elle y est inscrite sous le nom de Léios. trapu, qui servait à désigner ces deux jeunes animaux avant que M. Hallowell nous eût envoyé le sujet dont la croissance semble achevée, et avant que l'identité de nos exemplaires de petite taille et à livrée de jeune âge avec l'espèce déjà connue eût pu être établie. Ce dessin et celui que porte la même pl. xxn, fig. 2 (Léios. de Bell), sont destinés à montrer les différences qui se remarquent: $1^{\circ}$ dans la disposition des écailles sus-céphaliques, particulièrement de celles de la région inter-orbitaire, et $2^{\circ}$ dans la situation des narines bien plus rapprochées de l'extrémité du museau chez le Léios. à collier que chez le Léios. de Bell, originaire du Mexique, et ainsi nommé par mon père et par Bibron en l'honneur du savant naturaliste qui en a fait présent. Dans cette deuxième espèce, l'écaillure de la face supérieure de la tête n'est pas semblable à celle du Léios. à bandes rapporté par M. le professeur D’Orbigny et représenté dans la partie erpétologique de son Voy. Amér. mérid., pl. is, fig. ${ }^{4}$.

1. D’après les observations que j'ai présentées sur l'identité des Crotaph. et des Léios., il y a lieu d'inscrire sous ce dernier nom trois autres espèces qui ne font pas partie de nos collections, et décrites, la première, par MM. Baird et Girard : Crot. Wislizenit, provenant de New-Mexico (Stansbury's exploration of the valley of the great salt lake, 1852. Appendix Rept., p. 340, pl. m), la deuxième, par les mèmes zoologistes: Crot. Gambelii (Proceed. Acad. Philad. Août 1852), recueillie en Californie, et la troisième, par M. Hallowell : Crot.fascialus (Sitgreaves report of an expedit. down the $Z$ uni and Colorado rivers, $1853, \mathrm{p} .115, \mathrm{pl}$. v). Cette dernière espece, au reste, qui devient pour nous Leios. fasciatus, ou plutôt Leios. Hallowellii pour la distinguer nominativement du Leios. fasciatus, Dum. Bib., originaire de l'Amérique du Sud, diffère de ce dernier non-seulement par ses caractères spécifiques, mais par sa zone géographique, car elle a été prise sur les collines de sable de l'extrémité inférieure du Jornada del Muerto, New-Mexico. - Un quatrième Saurien des États-Unis et qui nous est également inconnu, est décrit par MM. Baird et Girard (Proceed. Acad. Philad. Archives du Muséum. T. VIII. 
XVI. GENRE (bis), DHPEOHEME. DRPLOLARUS. BELL.

(Zool. of the. Voy. of the Beagle, Rept. p. 19.)

Corps assez déprimé, sans crête, à écailles très-petites en dessus, presque circulaires, lisses et convexes; polygonales, à peine imbriquées, lisses et planes en dessous; un pli transversal et deux plis longitudinaux sous le cou; queue ronde, sans carènes, de longueur médiocre; tête courte, large et subtriangulaire, couverte d'écailles nombreuses, petites, arrondies, non imbriquées; oreilles àbords nonépineux; point de pores fémoraux ou pré-anaux ni dans l'un ni dans l'autre sexe; pieds courts et robustes; pas de dents palatines.

Ce genre, quoique très-voisin des Léiosaures, ainsi que le dit M. Th. Bell, en diffère cependant : $1^{\circ}$ par l'absence des dents au palais; $2^{\circ}$ par la brièveté proportionnelle de la queue ; $3^{\circ}$ par la disposition des plaques sousorbitaires qui, au lieu d'être distinctes et d'égale grandeur, comme dans les Léiosaures, sont irrégulières, car on en voit trois plus grandes que les autres, réunies entre elles et n'en formant, en quelque sorte, qu'une seule.

Ces différentes particularités sont suffisantes pour faire admettre la nouvelle coupe générique dont Bibron, dans un voyage à Londres, avait, ainsi que le savant zoologiste anglais, admis la nécessité.

XXVII. - I. Diplolème DE Bibrot, Diplolamus Bibronii, Bell. (loc. cit., p. 21 , pl. $\mathrm{xI}$ ).

Idem., Gr. Cat. of Liz., p. 225. - Id., Dum,, Cat. Rept. du Mus. de Paris, p. 68. - Id., Id., Rẻpert. Erpèt. génér., Dum. Bib., t. IX, p. 267.

Écailles de la tete planes; queue plus courte que la tele et le tronc réunis.

La tête est épaisse, rude, plus longue que large, à plaque occipitale trèspetite, plate et hexagonale; le museau est obtus; entre les plaques sus-

Août 1852, et figuré sur l'une des planches d'un ouvrage qu'on n'a pas encore reçu en France. Hs to nomment Crotaph. dorsalis; mais ce Reptile a été éloigné de ce genre par M. le docteur Hallowell qui a fait observer (Proceed of Acad. Philad. Juin 1854) qu'il diffère du Crotaphyte, Holbr., en ce qu'il a le corps couvert non de granulations, mais d'écailles quadrangulaires, et que sur la ligne médiane du dos, il a des écailles plus grandes et carénées, formant une petite crête dont aucune trace n'existe chez le Crotaphyte. Par ces différents motifs, il considère cette espèce comme type d'un genre nouveau: Dipsosanrus et elle devient D. dorsalis Hallowell. 
labiales et les sous-orbitaires, il y a trois ou quatre rangs de petites écailles. Le corps est large, déprimé, sans aucune crête ou saillie médiane, à écailles très-petites. La queue, légèrement triangulaire à sa base et conique à son extrémité, porte, chez les deux sujets adultes de nos collections, o ${ }^{\text {mi }}$ o 3 de moins que la tête et le tronc réumis, mais chez un jeune sujet, elle est à peu près égale à la moitié de la longueur totale de l'animal. Les membres ont des dimensions médiocres; les postérieurs, placés le long des flancs, ne s'étendent que jusqu'à l'aisselle.

La tête est d'un brun sombre, avec quelques taches plus foncées; la teinte générale du dos est un bleu gris taché de rouille, orné de cinq bandes transversales, comme dentelées à leur bord postérieur et formées par la réunion de petites taches rapprochées les unes des autres. Ces bandes sont entourées de blanc jaunâtre ou de jaune clair; elles se continuent sur la queue où elles forment des demi-anneaux. - Dans le jeune âge, le système de taches qui vient d'ètre décrit se retrouve complétement, mais la teinte générale est un brun jaunâtre.

Le IIuséum doit à la générosité de M. le docteur Bell trois exemplaires de cette espèce ( 2 adultes et un jeune), mais il ne possède pas celle que ce même zoologiste a décrite sous le nom de Dipl. cle Darwin, et qui a été recueillie comme la précédente au Port-Désiré (Patagonie) par le naturaliste dont elle porte le nom.

Cette dernière diffère du Dipl. cle Bibron en ce qu'elle a: I $^{\circ}$ les écailles de la tète convexes; $2^{\circ}$ un seul rang de squames entre les plaques labiales et les sous-orbitaires; $3^{\circ}$ toutes les pièces de l'écaillure un peu plus grandes, et $4^{\circ}$ enfin, la queue plus longue que la tête et le tronc réunis.

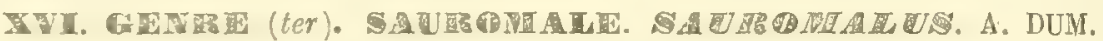

GLYRE NOUYEAU.

Corps très-déprimé, sans crêle, à flancs bordés d'un pli cutané; écailles petites, quadrangulaires, non imbriquées et disposées en rangées transversales régutières, tête aplatie, à plaque occipitale pelite; un pli en travers sous le cou qui, de chaque côté, en porle un autre demi-circulaire et garni d'écailles épineuses; pas de dents au palais; bord antérieur de l'oreille dentelé; des pores auso cuisses et non à la région anale; membres robustes, à doigts courts; queue longue et forte, déprimée à sn base et arrondie dans le reste de son étendue. 
L'un des caractères remarquables de ce geure se tire de l'aplatissement du tronc, d'où le nom par lequel je propose de le désigner et qui est formé des mots grecs aqupos, lézard, et oparos, plat. De tous les Sauriens pleurodontes à corps déprimé, il n'y en a pas qui se rapprochent plus de celui dont je m'occupe en ce moment que les Diplolèmes. Le Sauromale cependant se distingue d'une façon notable : $1^{\circ}$ par les plis latéraux du cou et des flancs; $2^{\circ}$ par la longueur proportionnelle plus considérable de la tête et de la queue; $3^{\circ}$ par la présence de dentelures au bord antérieur de l'oreille, et de pores sur la face interne des cuisses. Aussi le Saurien unique dans nos collections, qui offre ces différences remarquables, doit-il devenir le type d'un genre nonveau ne comprenant jusqu’à présent qu'une seule espèce.

XXVIII. - r. Sauromale sombre, Sauromalus ater, A. Dum. ESPÈCE NOUVELLE. - Pl. xxHI, fig. 3 et $3 a$.

plaques sus-céphaliques non imbriquées, lisses, toutes à peu près semblables entre elles pour les dimensions; narines circulaires, dirigées en haut et un peu en dehor's; écailles sans carènes; teinte générale d'un brun rougeâtre, relevée sur les flancs par de pelites taches noires irrégulières, peu apparentes.

Le tronc est large et déprimé; sur la ligne médiane du dos, il règne, depuis le cou, jusque sur la base de la queue, un petit enfoncement ou sillon où l'on voit s'infléchir d'avant en arrière, et s'entre-croiser un peu les rangées transversales des écailles; ces rangées semblent ainsi composées chacune de deux portions, l'une droite et l'autre ganche légèrement déviées de leur direction par leur rencontre au niveau de ce sillon.

De toutes les pièces de l'écaillure sus-céphalique, ce sont les sus-oculaires qui ont les plus petites dimensions. Immédiatement derrière les narines et en avant des régions orbitaires, il y a quelques plaques plus grandes que les autres. L'ouverture nasale un peu tubuleuse, est percée dans une seule plaque entourée de plusieurs petiles squames. Le bord antérieur de l'oreille est armé de quatre écailles épineuses, dont la troisième, en comptant de haut en bas, est la plus longue. Les paupières sont granuleuses; la ligne susorbitaire est revêtue d'un rang d'écailles rhomboïdales toutes semblables entre elles et planes. La ligne sous-orbitaire porte des écailles relevées en dos d'âne sur leur ligne médiane; elles sont aı nombre de quatorze, et les postérieures se dirigent jusqu'au bord supérieur du tympan. 
ORDRE DES SAURIENS. - IGUANIENS PLEURODONTES.

Il y a 28 à 30 plaques sus-labiales, sans rostrale médiane et impaire, plus longues que hautes; les médianes sont les plus basses. Elles sont surmontées de plusieurs rangs de petites écailles. On compte 27 souslabiales, en y comprenant la mentonnière, qui a la forme d'un triangle allongé à sommet arrondi.

Les écailles du cou diffèrent de celles du dos en ce qu'elles ne sont pas quadrangulaires et planes comme elles, car elles ont l'apparence de petits tubercules très-serrés et à sommet pointu. L'écaillure de la gorge se compose de fines granulations fort nombreuses. Les squames du ventre sont lisses, moins volumineuses que celles du dos, et forment des rangées horizontales régulières. La queue est revètue d'écailles disposées en verticilles. Sur sa base, jusqu'à l'extrémité du sillon médian dont j’ai déjà parlé, elles sont lisses, mais à partir de ce point, on voit sur le milieu de chacune d'elles, en dessus comme en dessous, une carène arrondie plus saillante à l'extrémité postérieure de l'écaille qu'à l'antérieure, et plus particulièrement prononcée vers le bout de la queue. Les pièces de l'écaillure des membres l'emportent sur toutes les autres par leurs dimensions. Sur les membres antérieurs et sur les cuisses, elles sont lisses, et au contraire tuberculeuses sur la région postérieure des jambes et des pieds, ainsi que sous tous les doigits.

Le pli cutané de la partie latérale du cou décrit une demi-ellipse à concavité antérieure; ses extrémités vont se perdre l'une au-dessus du conduit auditif, et l'autre au-dessons de la mâchoire inférieure; son pourtour, en forme de bourrelet, est revêtu d'écailles assez volumineuses, terminées en pointe, et il est surtout saillant à sa partie moyenne. Le pli transversal du con se prolonge en haut et en arrière, pour aller se terminer sur le dos au niveau des épaules, qui sont, en outre, entourées par deux autres plis moins prononcés.

Il y a, le long de la face interne de chaque cuisse, I 4 à 15 pores.

Je n’ai en quelque sorte rien à ajouter aux indications relatives au système de coloration données dans la diagnose. On est frappé tout d'abord de la teinte sombre de ce reptile, et c'est ce qui m’a déterminé à le désigner par cette épithète (Saurom. ater). Les régions orbitaires sont moins foncées que le reste; elles ont une nuance brun-jaunâtre; les régions inférienres sont plus claires que les supérieures. Quant aux taches des flancs, elles sont petites, irrégulières et peu visibles. 
La longueur totale est de $o^{\mathrm{m}} 265$ ainsi répartis : tête et tronc, $o^{\mathrm{m}} \mathrm{r}_{3} \mathrm{o}$, queue, $\mathrm{o}^{\mathrm{m}} 135$.

L'espèce ne nous est connue que par un seul spécimen, dont nous ignorons l'origine, et qui a été donné au Muséum par M. Jaurès, lieutenant à bord de la frégate la Danaïle.

- Les genres Upéranononte Dum. Bib. et Hypsibate Wagler (1 7 et 18), tels qu'ils sont constitués dans l'Erpét. génér., n'ont subi depuis 1836 , aucune modification qu'il soit important de rappeler ici. Les changements de dénominations proposés sont indiqués plus haut dans les analyses que j'ai présentées des systèmes de classification de MM. Fitzinger et Gray.

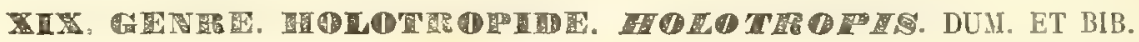

De nouvelles espèces rapportées à ce genre, qui a reçu de M. Gray le nom de Léiocéphale, ont été signalées dans ces derniers temps. Je dois faire connaître celles que notre Musée possède ${ }^{1}$.

XXIX. - 2 bis. Holotropide de Gray, Holotropis Grayii, Dum.

Leioceph. Gr., Bell, Foy. of the Beagle Rept, p. 24, pl. xiv, fig. 1. - Idem, Gr. Cat. of Liz., p. 218. - Hol. de Gr., Dum., Cat. des Rept. du Mus. de Paris, p. 70. - Id., Répert. Erpét. génẻr., Dum. Bib., t. IX, p. 268.

Écailles du ventre rhomboïdales, non carénées; écailles sus-céphaliques lisses, et l'occipilale grande; sur les régions sus-orbitaires, de larges plaques précédéés d'écailles beaucoup plus petites et imbriquées; au bord antérieur de l'ouverture de l'oreille, quatre dentelures.

La crête dorsale, sans être précisément élevée, est cependant moins basse que celle de l'Holotr. microlophe, 'c'est un caractère distinctif à joindre à ceux qui sont fournis par les grandes dimensions, non-seulement de la plaque occipitale, qui est pentagonale et un peu échancrée à son bord postérieur, mais des quatre ou cinq plaques transversales des régions sus-orbi-

1. Une modification a été apportée par M. Gray à la synonymie de l'Hol. de Lherminier que les auteurs de l'Erpét. génér. avaient considéré comme identique à son Léiocéphale caréné; mais chez ce dernier, les écailles ventrales sont lisses (Cat. of Liz., p. 217), tandis que dans l'autre espèce, elles sont carénées. Ces Sauriens sont donc différents l'un de l'autre, et doivent être distingués par les deux dénominations que jo viens de rappeler. - Outre ce Léiocéphale, M. Gray en a fait connaître deux autres à écailles rentrales lisses, et qui nous sont inconnus; il les nomme L. Mac-Leayiz et $L$. ornatus. - Nous possédons à la Ménagerie, depuis plus d'une année, plu-

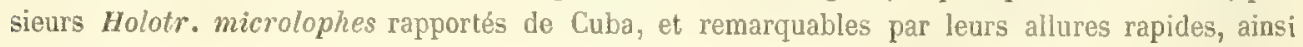
que par la facilité avec laquelle ils supportent leur captivité, tout en restant craintifs et farouches. 
taires. Les autres écailles du dessus de la tête sont assez grandes et légèrement bombées, mais non carénées, ce qui établit une distinction de plus et fort importante avec l'Hol. tête-rude. Il en est de même pour les dentelures de l'orifice tympanal. Les écailles des tempes sont faiblement carénées et non imbriquées; celles du dos, surmontées chacune d'une forte carène, pointue à son extrémité, sont disposées en séries longitudinales nombreuses, convergentes en arrière, vers la crête dorsale, qui est formée d'écailles plates, verticales et prolongées de la nuque à l'extrémité de la queue.

Le système de coloration très-altéré sur nos individus est ainsi décrit dans les notes de M. Darwin, citées par M. Bell : Parties supérieures d'un brun clou de girofle, passant au noir brun, avec des taches noires souvent disposées en bandes transversales ou longitudinales; flancs légèrement nuancés d'une teinte orangée; quelques-unes des écailles de la crête, près de la tête, blanches; ventre presque blanc, et toute la gorge d'un noir éclatant.

Longueur totale du plus grand individu : $0^{\mathrm{m}} 23$ ainsi répartis : tête et tronc, $\mathrm{o}^{\mathrm{m}} \mathrm{og}$, queue, $\mathrm{o}^{\mathrm{m}} \mathrm{i} \mathrm{c}$.

Les échantillons rapportés à Londres ont été pris par M. Darwin, dans les îles Galapagos. C'est sans doute aussi de cet archipel que proviennent les trois individus donnés au Muséum par M. Nibou.

XXX. - 2 ter. Holotropide TÊTE-RUDE, Holotr. trachycephalus, A. Dum. Pl. xxiII, fg. 4 .ef 4 a.

Itom, Idem, Cat. Rept. Mus. de Paris, p. 70. - Id., Id., Répert. Erpét. génér., Dum. Bib., t. IX, p. 268.

Écailles ventrales lisses; celles de la tête petites, inégales, carénées et un peu rugueuses; celles des régions sus-orbitaires irrégulières et nombreuses; plaque occipitale petite.

La crête, moins haute que celle des $\boldsymbol{H}$. de Lherminier et $\boldsymbol{H}$. de Gray, est cependant plus élevée que chez l' $H$. microlophe. Les écailles du tronc, dont les dimensions sont moindres que dans ces trois espèces, portent une carène plus petite, et elles forment des lignes moins obliques et par suite moins convergentes vers la région moyenne du dos. Les plaques de la tête sont carénées, et quoique ce caractère se retrouve chez l'H. de Lherm. et dans l'espèce nommée par M. Gray, Leioceph. ornatus (Cat. of Liz., p. 2 I9), inconnue au Musée de Paris, celle que je décris ici s'en distingue très-faci- 
lement, car l'H. de Lherm. a des écailles sus-orbitaires larges et multicarénées, et le $L$. $(H$.$) orné a les écailles de la nuque plus petites que celles$ du tronc, une crête élevée, de larges bandes noires en travers sur le dos, et une tache également noire au-devant de chaque épaule. - Le cou de l'H. téte-rude est un peu plissé latéralement et en travers. - La crête dorsale se continue, en diminuant progressivement de hauteur, sur la queue, dont les dimensions sont assez considérables, et qui est robuste et comprimée.

La teinte générale est un vert olive, relevé sur les flancs par un piqueté d'un vert plus clair, par des taches brunâtres plus ou moins apparentes, puis par une raie longitudinale d'un vert tirant sur le jaune, et plus visible chez les femelles que chez les mâles, qui portent un large demi-collier noir sous le cou, et ont quelquefois le ventre de la même teinte foncée que la région gulaire.

Cette espèce nous est connue par de nombreux exemplaires des deux sexes rapportés de la Nouvelle-Grenade, et en particulier de Santa-Fé de Bogota par M. J. Goudot. - Le plus grand spécimen a une longueur totale de $\mathrm{O}^{\mathrm{m}} 23$ ainsi répartis : tête et tronc, $\mathrm{o}^{\mathrm{m}} \mathrm{09}$, queue, $\mathrm{O}^{\mathrm{m}} \mathrm{I} 4^{4}$.

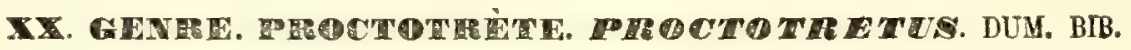

Aux espèces de cette division déjà nombreuse en 1837 , ainsi qu'on le voit dans l'Erpét. génér., où mon père et Bibron en ont décrit dix, dont huit jusqu'alors inconnues, les travaux récents des naturalistes en ont ajouté plusieurs, toutes originaires, comme les premières, de la côte occidentale de l'Amérique du sud. Parmi ces espèces nouvelles, il n'y en a que trois dans nos collections. Les autres n'y sont point encore parvenues .

1. Le genre Holotropide ou Léiocéphale, dont il est ici question, fait partie dans le Syst. de M. Fitzinger, ainsi qu'on l'a vu précédemment (p. 507) de la petite famille des Hétératropides qui, outre ce genre et celui que Cuvier a nommé Ecphymote, lesquels n'ont pas la queue épineuse, contient les trois suivants, dont les écailles caudales sont plus ou moins prolongées et pointues : Sténocerque Dum. Bib., Trachycycle Id., et Strobilure Wiegm. C'est dans cette famille que M. Tschudi (Fauna peruana, Rept., p. 25-29) place de nouveaux Iguaniens que nos collections ne possèdent pas. Voici comment ce zoologiste les classe et les nomme :- AMBLYGLOSSA. Ordo II HuMivagx. Fam. I Heterotropides. - I Gen. Steironotus Fitz. I subgen. Eulophus Tsch. 1 St. arenarius Tsch. - II Gen. Scelotrema Tsch. 1 Sc. formosum Tsch. pl. I, fig. 1; 2 Sc. crassi-caudatum Tsch.

2. Tels sont les Proct. de Bibron, de King, de Darwin Bell (Rept. in Zool. of the voy. of Bea-

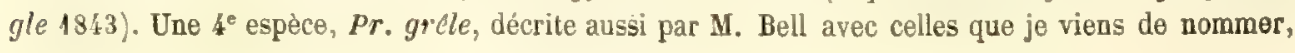




\section{XXXI. - I bis. Proctotrète mosaïque, Proctolretus mosaïcus, Hombron et Jacquinot.}

Idem, Hombr. et Jacq., Atlas Voy. au pole S. et Océanie Comm. Dum. d'Urville, Saur., pl. Ir, fig. 1 et $\mathbf{A}, a$, texte de M. Guichenot.

P. interméd. au P. du Chili et au P. ventre-bleu, Atl. Voy. de la Vénus, pl. II, fig. 1 et 4 a. P. mosaïque Guich. Rept. Hist. Chili, Cl. Gay, p. 26. - Id., Dum. Cat. Rept. Mus. Par., p. 72. - Id., Id., Répert. Erpét. génér., D. B. t. IX, p. 269.

Cou un peu plissé sur les cotés; plaques sus-céphaliques non imbriquées, ni carénées; une seule rangée d'écailles entre les plaques sus-labiales et les sous-orbitaires; bord antérieur de l'oreille légèrement dentelé; face postérieure des cuisses tout à fait granuleuse; deux raies jaunes de chaque coté du corps, séparées entre elles par un grand nombre de petites taches noires, qui manquent sur la région médiane du dos, où la teinte brune du fond forme une bande longitudinale.

Des différentes plaques du museau, celles qui viennent immédiatement après la rostrale sont les plus petites; elles en précèdent six autres, trois de chaque côté, entre lesquelles il s'en trouve une ou bien deux placées l'une au-devant de l'autre. Derrière ce groupe, on en voit d'ordinaire une médiane, qui semble réunir les régions sus-orbitaires, dont l'écaillure est formée par trois ou quatre grandes plaques plus larges que longues, et précédées de plusieurs écailles beaucoup plus petites; de grandes plaques bordées par un cercle intérieur de petites squames, forment l'entourage de ces régions susorbitaires. Derrière l'occipitale, qui est petite, il y a deux grandes plaques.

nous a été donnée par lui. Dans cet ouvrage, il a complété d'une manière fort intéressante le chapitre de l'Erpét. gẻnér., relatif au genre dont il est question, en consacrant les pl. $\mathbf{r}$ à $\mathbf{x}$ de l'Atlas de ce Voyage à toutes les espèces de Proctotrètes rapportées par M. Darwin au retour de l'expédition. Or, dans cette précieuse collection, M. Bell a retrouvé non-seulement les $P$. du Chili et à taches noires signalés d'abord par Lesson et par Wiegmann, mais encore toutes les espèces nouvelles décrites par mon père et par Bibron, à l'exception du $P r$. signifère, qui a été dessiné d'après le type du Musée de Paris. Les quatre espèces dont la connaissance est due à M. Bell, sont également figurées sur ces planches. - De bonnes représentations des Proct. du Chili, ventre-bleu, peint et svelte se voient dans I'Atlas des Rept. in Hist. du Chili de Cl. Gay, joint aux descriptions faites par M. Guichenot, et dans la Zool. du Voy. de la Vénus, Comm. Du Petit-Thouars.-Je ne dois pas omettre de citer les autres Pr. inconnus au Musée de Paris. Aux trois sous-genres Proctotretus, Leiodeira et Liolæmus établis par M. Fitz. dans le genre Liolæmus Wiegm., M. Tschudi (Fauna peruana,p. 34) en a ajouté un $4^{e}$ Sauridis (Liol. (S.) modestus); et il a décrit un Liolæmus elegans Tsch. (p. 33), - Il y a, en outre, Pr. femoratus et Stantoni Girard, des environs de Santiago (Proceed. Acad. of Philad. Nov. 1854). - Relativement au $P r$. de King, M. Bell pense que peut-être il faudrait y rapporter les individus du Pr. de Fitz. Dum. Bib. décrits comme types des Var. A et B de cette dernière espèce. Ne connaissant pas le $\boldsymbol{P r}$. de King, je ne puis que mentionner cette supposition du zoologiste anglais. Aachives dU MuséUM. T. VIII. 
On compte, à la mâchoire supérieure, 6 ou 7 plaques allongées, de chaque côté de la rostrale, dont la hauteur est aussi considérable que celle d’es plaques sus-labiales et que celle du rang unique d'écailles qui les surmontent; à la lèvre inférieure, il y a gou u petites squames, en y comprenant la mentonnière qui est grande. - Les écailles du tronc et des membres n'offrent rien de particulier à noter, si ce n'est qu'elles sont peu développées. - La queue est longue, grêle et très-effilée à sa pointe.

La teinte générale, en dessus, est un brun verdâtre, qui forme une bande uniforme le long de la ligne médiane, mais sur les parties latérales du dos, le fond disparaît presque complétement sous un grand nombre de petites taches noires, les unes transversales, les autres longitudinales, représentant une sorte de mosaique, et au milieu desquelles on voit deux raies longitudinales d'un brun jaunâtre clair. Des lignes noires étroites parties du pourtour de l'orbite se dirigent, les unes en bas, et les autres en arrière.

Ce Proct., qui est un des plus petits du genre, doit venir immédiatement après le $P r$. du Chili, parce qu'il a les plis du cou très-peu marqués. Son système de coloration, ainsi que les différentes particularités indiquées dans la diagnose et dans la description s'opposent à ce qu'il soit confondu avec ses congénères.

Patrie: Chili. MM. Cl. Gay, Gardichaud, Darwin, nous ont donné plusieurs individus. De Talcahueno, en particulier, nous en arons recu de MM. Hombron et Jacquinot, et de Valparaiso, par M. Dubois.

XXXII. - 4 bis. Proctotrète grête, Proclolretus gracilis, Bell. Zool. of the Voy. of the Beagle, p. 4, pl. I, fig. . .

Leiodera gracilis, Gr., Cat. of Lis, p. 211.-Pr.gracilis, Dum. Cat. Rept. Mus. de Par., P. 73. Id., Id., Rẻpert. Erpét. gênér., D. B. t. IX, p. 269.

Corps grele; écailles de la tête lisses, non imbriquées; bord antérieur de l'oreille portant deux ou trois petits tubercules; cou à écailles imbriquées et à plis latéraux très-peu apparents; une seule série d'écailles au-dessus des plaques labiales; face postérieure des cuisses entièr"ement granuleatse; quatre raies longitudinales ja unes sur le tronc.

La tête est courte; chacune de ses faces latérales forme à peu près un triangle équilatéral; elle est recouverte de plaques assez grandes, rangées derrière les narines en quatre séries composées, la $1^{\text {re }}$ de 2 pièces, les $2^{\mathrm{e}}$ et $3^{\mathrm{e}}$ de 3 pièces, et la $4^{\text {e }}$ de 2 seulement. L'occipitale est petite et entourée de plaques moins grandes encore, à l'exception de deux de ces plaqries de 
forme pentagonale qu'elle touche par ses bords latéro-postérieurs disposës en angle. - Il n'y a qu'un senl rang de squames beaucoup plus longues que hautes entre l'bil et les sus-labiales, qui sont au nombre de six et ont la même forme allongée. La rostrale est également basse. Les souslabiales sont semblables aux précédentes; on en compte cinq de chaque côté de la mentonnière, qui a des dimensions plus considérables. - Les écailles du dos sont petites, rhomboïdales et à carène peu saillante; celles des aisselles et de la face postérieure des cuisses sont granuleuses.

La queue a une longuenr presque double de celle de la tête et du tronc réunis; les membres sont bien développés.

La teinte générale des parties supérieures est un brun grisâtre; une raie jaume longitudinale s'étend, de chaque côté, depuis le bord supérieur de l'orbite jusque vers l'origine de la queue; une autre raie semblable et parallèle à la précédente, part du bord inférieur de l'oeil et cesse au niveat de la cuisse. Les flancs sont tachetés de noir, ainsi que la mâchoire inférieure.

Le système de coloration établit une différence bien tranchée avec le $P r$. svelte, car celui-ci, qui offre une assez grande ressemblance dans sa conformation générale avec le $P r$ grêle, n'est pas rayé de jaune; en outre, on voit sur les côtés du cou du Pr. suelte, des écailles granuleuses, tandis que dans la nouvelle espéce, elles sont imbriquées et semblables à celles du reste du corps. Il faut noter enfin, comme bon caractère distinctif de ce $P r$., que les plis du cou, bien qu'ils ne manquent pas complétement comme chez le $P r$. clu Chili, sont cependant beaucoup moins apparents que chez la plupart des autres espéces de ce genre. - Le spécimen unique de notre collection a été donné par M. Bell. Il provient des collections faites au Chili par M. Darwin.

\section{XXXIII. - 8 bis. Proctotrète de Mageldan, Proctolrelus Magellanicus, Hombron et Jacquinot.}

Idem, Hombr. et Jacq. ( Toy. au póle sud et dans l'Océanie, Rept. Saur., pl. II, fig. 2 et B, b b', texte de M. Guichenot, p. 6.

Idem, Cat. Rept. Mus.de Par., p. 7อ.. - Id., Répert. Erpét. génér., D. B., t. IX, p. 269.

Corps trapu; tete petite, à musean obtus; plaques sus-céphaliques non imbriquëes, ni carénées; deux tubercules sur le borl antérieur de l'oreille; cótés du cou plissés et à écailles imbriquées; une seule rangée d'écailles entre le bord sous-orbitaire el les plaques labiales; face postérieure des cuisses entierement granuleuse; sur les régions supérieures, cinq raies longitudinales llanckes, séparées par des taches noires qui sont bordées de blane. 
Les formes sont ramassées et les membres courts. Les plaques sus-céphaliques antérieures ne sont pas carénées, mais elles sont bombées et un peu saillantes; leur disposition n'est pas tout à fait aussi régulière que dans l'espèce dont je viens de donner la description. L'occipitale est petite, moins cependant que les plaques environnantes, à l'exception des deux qui la suivent immédiatement. - Les écailles surmontant les labiales sont plus basses que celles-ci, dont on compte six de chaque côté de la rostrale qui est assez grande. Les sous-labiales, au nombre de cinq, sont plus longues que hautes, surtout les postérieures. - Les écailles ne sont pas trèsgrandes, et leur carène est peu saillante. - La queue, assez grêle et effilée à sa pointe, est seulement un peu plus longue que la tête et le tronc réunis.

La teinte générale est un brun verdàtre relevé par les cinq raies qui parcourent dans sa longueur la région dorsale. La médiane, bifurquée sur le museau, est la plus étroite; elle disparaît sur la base de la queue, et coupe, sur la ligne moyenne, sept taches noires irrégulièrement quadrilatères, à bord postérieur d'un blanc jaunâtre. Ces taches sont limitées en dehors par une raie claire prolongée depuis l'arcade sus-orbitaire jusque sur les parties latérales de la queue. Cette raie còtoie, du côté externe, une autre série de six taches noires pareilles à celles que je viens de décrire et qui sont en contact, sur les flancs, avec la raie la plus externe, dont la couleur est semblable à celle des précédentes. Cette dernière raie latérale commence derrière l'oeil et à la région gulaire par une bifurcation, qui cesse au niveau de l'épaule, puis elle s'étend jusqu'à la racine de la cuisse, et l'on voit au-dessous d'elle une troisième série de taches noires disposées avec autant de régularité que les deux autres, d'où il résulte que le dos est traversé d'un côté à l'autre dans toute sa longueur, par sept bandes noires interrompues. Sur la queue, il y a trois raies longitudinales. Les membres sont tachetés de noir en dessus. - Toutes les régions inférieures, excepté à la queue, ont une teinte noirâtre sur laquelle se détache en clair l'extrémité libre de chaque écaille, ce qui fait paraître le dessous de l'animal comme moucheté de ver sur un fond sombre. La gorge est moins foncée, car on y voit seulement des lignes noires sinueuses et disposées en chevrons à sommet postérieur.

La description qui précède suffit pour montrer les différences qui distinguent ce Proctotrète de tous ses congénères. Le spécimen type du Musée de Paris est évidemment adulte; on ne peut donc pas supposer avec M. Gray 
(Catal., p. 2 I5) qu'il appartient à l'espèce dite Pr. de King, dont il représenterait, suivant lui, le jeune âge. Cette hypothèse est basée sur la similitude qui se remarque entre ce $\operatorname{Pr}$. de Magellan et le Saurien que M. Bell a fait figurer sous le $\mathrm{n}^{\circ} 2$ de la pl. vi (Voy.du Beagle), mais avec cette note que cet individu diffère assez du $P r$. de King, pour lui faire croire qu'il appartient à une espèce distincte. On ne saurait, en effet, douter que le dessin de ce $P r$. $\mathrm{n}^{\circ} 2$ ne se rapporte à une autre espèce. Or, c'est évidemment à celle dite $P_{r}$. de Magellan qu'il convient, car celui-ci, outre les dissemblances du système de coloration, diffère encore du $P r$. de King par l'écaillure complétement granuleuse de la face postérieure des cuisses, caractère qui manque chez ce dernier. - Notre spécimen unique a une longueur totale de $o^{\text {ma }} \mathbf{I}_{2}$ ainsi répartis : tête et tronc, $o^{\text {nz }} 06$, et queue, $o^{m} 06$.

Comme tous ses congénères, ce Saurien vit dans la partie méridionale de l'Amérique du sud. Il a été rapporté des còtes du détroit de Magellan par M.M. Hombron et Jacquinot.

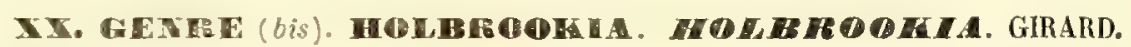

Stansbury's explorat. of the valley of the great salt lake of Utah, Rept., p. 341.

Cophosaurus Troschel, Arch. für Naturgesch, portant la date de 1850 , I, mais publié en 1852 seulement.

Tympans non visibles. Tête couverte de petites plaques polygonales; pas de dents au palais; un pli cutané sous la gorge; des pores fémoraux, mais pas de pores anaux; écailles peliles, légèrement imbriquées.

Ce genre, si remarquable par l'absence de toute indication extérieure des organes de l'audition 1, nous est connu par trois exemplaires reçus de l'Académie de Philadelphie, par l'obligeante entremise de M. le docteur Hallo. well. Nous avons, en outre, de jeunes individus recueillis au Mexique, et donnés par M. Trécul.

On peut, avec M. Girard, comparer jusqu'à un certain point le Saurien dont il s'agit aux Proctotrètes, à cause de son apparence générale; il présente

1. Cette particularité est rare chez les Sauriens, car elle ne se rencontre que chez les Caméléons, chez les Iguaniens appartenant aux genres Otocrypte et Phrynocéphale, chez les Dragons rayé et Spiloptère ou Dragonneaux de Wiegmann, et enfinchez les Glyptodermes ou Amphisbéniens et chez les Orvets, qui malgré ce caractère et malgré l'analogie remarquable de leur conformation exté rieure avec celle des serpents, sont cependant de véritables Sauriens. 
aussi quelque ressemblance avec le Tropidolépide microlépidote; il est inutile, au reste, d'insister sur ces analogies, qui motivent son classement entre les deux genres que je viens de nommer, mais avec lesquels aucune confusion n'est possible, en raison de cette particularité tout à fait exceptionnelle que ses tympans sont cachés.

On connait maintenant quatre espèces. Il y en a trois qui ne sont pas au Musée de Paris : H. texana Baird et Gir. (Cophosaurus texanus Troschel); H. affinis B. et G., H. propinqua B. et G. (Proceed. Acad. Philad., août 1852), mais nous possédons l'espèce type.

XXXI7. - I. Holbrookia tachetée, Holbr. maculata, Gir.

Idem, Gir., Proceed. Amer. assoc. advancem. of sc. Iv (1850) 1851, p. 201; Stansbury's explorat., p. 342, pl. vi, fig. 1-3, et Nat. hist. Red river, p. 326.

Queue à peu près égale en longueur au tronc. Tête sub-circulaire, légèrement.conique en avant; bord libre du pli pectoral garni de grandes écailles.

Les formes sont un peu lourdes et ramassées, surtout chez les femelles; les mâles et les jeunes sont plus élancés. Le tronc est sub-cylindrique, la queue conique et large à sa base. La tête plus large que haute, à museau tronqué, est couverte de plaques irrégulières, dont les moins petites occupent la ligne médiane entre les régions sus-orbitaires. La crête surciliaire est formée par deux ou trois rangs serrés d'écailles peu volumineuses et allongées; au bord sous-orbitaire, elles sont moins nombreuses, mais plus grandes, et la deuxième, en particulier, l'emporte sur toutes les autres par ses dimensions; on en voit enfin de petites et un peu pointues au bord libre des paupières, qui paraissent ainsi comme dentelées. Les plaques sus-labiales, au nombre de 7 de chaque côté de la rostrale, sont allongées et offrent une disposition assez remarquable en ce qu'elles sont obliques et imbriquées; il n'en est pas de même à la lèvre inférieure où l'on compte, en y comprenant la mentonnière, I 7 plaques quadrilatères et verticales, dont les plus grandes sont les plus rapprochées de l'angle de la bouche. Les tempes sont couvertes de squames qui ne laissent apercevoir aucune trace du tympan qu'elles recouvrent, et sont semblables à celles du cou, dont les parties latérales portent chacune un pli qui, se dirigeant en bas, vient se terminer à la région sons-maxillaire; au delà, mais à une fort petite distance, on voit le pli transversal très-prononcé du cou situé immédiatement au-devant des épaules. 
Les écailles du tronc sont très-faiblement imbriquées et carénées sur le dos et lisses sur le ventre. Sur la queue, elles forment des verticilles.

Les membres sont peu développés, mais surtout les antérieurs; les doigts sont allongés, complétement revêtus d'écailles. Sur chaque cuisse, on voit quatorze pores.

La couleur générale, suivant un dessin fait par M. W. H. Tappan, est, dit M. Girard, un brun olive, légèrement violacé sur les côtés de la tête. Sur l'un et l'antre flanc, il y a deux, et quelquefois trois taches foncées. Le dos est orné, de chaque côté de la ligne médiane, d'un rang de taches noires irrégulières. En dehors, i] y a, chez les mâles, une autre série de taches, mais moins apparentes, et chez les femelles, conformément à la pl. vi annexée à la description de M. Girard, ce sont de simples marbrures.

Sur la queue, les taches se continuent et ne tardent pas à se réunir en une série unique. Le système de coloration ne parait pas être différent dans le jeune âge de ce qu'il est à une époque plus avancée de la vie; en outre, la différence de sexe indiquée plus haut et tirée du nombre de rangées longitudinales de taches, est đéjà très-manifeste.

D'après Jes indications de M. Girard, l'animal reste petit, et en effet notre sujet adulte ne mesure en tout que $\mathrm{o}^{\mathrm{m}}$ ogo ainsi répartis : tête et tronc, $\mathrm{O}^{\mathrm{m}} \mathrm{0} 55$, queue, $\mathrm{o}^{\mathrm{m}} \mathrm{o} 35$.

Nos échantillons proviennent, les uns du Texas, et les autres du territoire des Cherokees (Tenessee États-Unis).

- Le $2 \mathrm{r}^{e}$ genre Tropidolépide Tropidolepis, Cuv. (Sceloporas Wiegm.), où ne sont comprises que des espèces de l'Amérique du nord, est un de ceux qui ont reçu le plus d'additions dans ces dernières années par suite des travaux des zoologistes des Étals.Unis sur les reptiles fort nombreux et très-intéressants de leur vaste territoire. Beaucoup de ces animaux manquent dans nos collections, et je dois me barner à en présenter une liste ${ }^{4}$.

1. Ces espèces sont: Sceloporus Poinsetii (Sc.torquatus, var. B. Wiegm.?), S. Clarkii, S. Thayerii, S. dispar, Baird et Gir. (Proceed. Acad. Philad. Août 1852); S. gracilis, occidentalis, froutalis; B. et G. (Id. Oct. 1852); S. graciosus, B. et G. (Stansbury's explorat., p. 346, pl. v, fig. 1-3, 1852); S. marmoralus, $S$. delicatissimus Hallowell (Proceed. Ac. Philad. Oct. 1852, et Sitgreaves expedit., p. 109 et 110 ); S. consobrinus Bi et G. (Nat. hist. of the Red river of Lousiana, 1853, D. 236 , pl. $\mathrm{x}$, fig. nombre des espèces plus anciennement décrites : $S$. horridus Wiegm.; $S$. æneus Id., et $S$. grammicus, Gr. sont encore inconnus au Muséo de Faris. 
Parmi les espèces établies par Wiegmann, il s'en trouve une qui diffère notablement de ses congénères par la petitesse des écailles, et c'est ce caractère remarquable que le savant zoologiste de Berlin a voulu exprimer en la nommant Scelop. microlepidotus. Or, cette particularité se retrouvant chez d'autres Sauriens très-voisins de celui-ci, également originaires de l'Amérique septentrionale, et tous plus petits que les vrais Scélopores, MM. Baird et Girard ont, avec raison, considéré l'espèce dont il s'agit comme type d'un genre spécial caractérisé par le peu de grandeur des écailles du tronc, contrastant avec les dimensions beaucoup plus considérables des écailles de la queue. Ils ont employé le mot Ura comme dénomination générique. Le Scelopore microlép. était inconnu aux auteurs de l'Erpét. génér. à l'époque de la publication du t. IV de cet ouvrage, mais nous l'avons reçu ultérieurement du Mexique par les soins de M. Ghuisbreght, et il a été possible de constater les différences assez notables qu'il présente quand on le compare aux espèces près desquelles il avait été rangé jusqu'à présent. Il devient Uta microlepidota B. et G.; on doit en rapprocher Uta Stansburiana Id. (Stansbury's explorat. great. salt. lake of Utah, p. 345, pl. v, fig. 4, 5 et 6 ), et $U$. ornata Id. (Proceed. Acad. Philad., août r 852), espèces que nos collections n'ont pas encore reçues.

- Relativement aux véritables Tropidolépides du Muséum, je n'ai rien de particulier à en dire, et je me borne à rappeler que j’ai fait connaître, en $185 \mathrm{r}$, les publications récentes qui les concernent (Cat. Rept. Mus. de Par., p. 76 et 77 ).

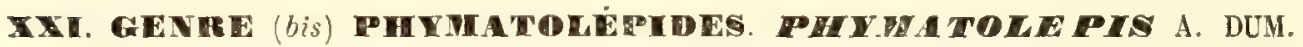

$$
\text { GENRE NOLYEAU. }
$$

Tronc sans crête, cóvvert en dessus de fines granulations juxtaposées, entremêlées de grandes écailles carénées; queue assez forte, et dont les écailles portent une carène; tête courte; plaque occipitale et sus-oculaires grandes; pas de dents palatines; un double pli sous le cou, qui est plissé latéralement; des pores fémoraux; pas de pores anaux.

Le caractère le plus remarquable se tire de l'aspect de l'écaillure des régions supérieures et latérales, qui est composée de petites squames presque circulaires, un peu bombées, non imbriquées et au milieu desquelles les 
ORDRE DES SAURIENS. - IGUANIENS PLEURODONTES.

écailles carénées se montrent comme de petites élévations tuberculeuses; d'où le nom dont je fais usage pour désigner ce nouveau genre, et qui est

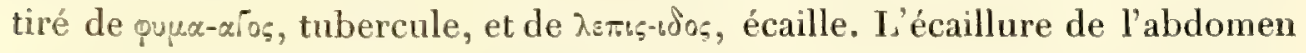
est fort différente, car il y a imbrication des squames assez grandes et lisses, dont elle se compose. - La tête est petite et le museau court; les plaques sus-céphaliques sont grandes. Les narines s'ouvrent en dessus. Le bord antérieur du trou anditif porte quelques dentelures. - Les écailles des membres sont fortement carénées. On voit sous la base de la queue, au delà du cloaque, chez le mâle, deux grandes écailles un peı concaves.

Parmi tous les Iguaniens pleurodontes, les Tropidolépides sont ceux qui ont le plus de rapport avec ce nouveau genre; c'est particulièrement à l'espèce dite $T$. microlépidote, et devenue l'un des types du genre Uta B. et G., qu'il faut le comparer. On voit alors mne grande analogie dans la plupart des caractères antres que ceux qui sont fournis par la disposition remarquable de l'écaillure du Phymatolépide qui, par cette particularité, s'éloigne forcément des divers groupes établis dans la famille des Iguaniens pleurodontes. Une seule espèce de ce genre est conservée au Musée de Paris.

XXXV. - I. Phymatolépide deutx-Carènes, Phymat. bi-carinalus A. Dum. Espèce xouvelle. - PI. xxiIr, fig. 2, $2 a$ et $2 b$ montrant le dessus de la tète et une-portion des téguments amplifiée.

Sur le dos, une double carène peu élevée constituée par deux séries longitudinales de grandes écailles très-rapprochées, longeant, à droite comme à yauche, la ligne médiane du dos, et formant, de chaque cóté de la colonne vertébrale, une ligne saillante qui commence au niveau des épaules, et se prolonge sur la queue; sur un fond brun verdatre, des taches noires irrégulières, plus hautes que larges, representant sur la nuque une porlion de collier, et sur la queue, des demi-anneaux étroits et uniformément espacés.

Le tronc est assez cléprimé; la queue est longue et robuste; les membres sont peu développés, surtout les antérieurs; la tête est courte et le museau obtus. -- En dehors de chacune des séries longitudinales de grandes écailles à carène saillante indiquées dans la diagnose, on en voit d'autres, dont la configuration et la grandeur sont semblables, mais beaucoup plus espacées entre elles, et qui, dans leur ensemble, forment une série parallèle à la précédente; les flancs portent, çà et là, quelques écailles également carénées et disposées sans ordre. Il y a, sous la gorge, un pavé granuliforme séparé des squames plus grandes et imbriquées de l'abdomen par les deux plis transAachives du Muséum. T. VIII. (1856) 
versaux du con, qui sont parallèles entre eux, et dont le bord postérieur porte des écailles terminées en pointe, plus développées que les antres. - I'écaillure sus-céphalique est disposée comme il suit : deux paires de petites plaques inter-nasales suivies de six autres plaques, dont deux médianes placées l'une au devant de l'autre; puis, trois grandes représentant ensemble une sorte de triangle à sommet antérieur et sitnées entre les régions susorbitaires qui, outre quatre grandes plaques transversales, sont recouvertes en avant et en dehors par de petites squames. L'occipitale est grande, presque quadrilatère, bordée sur les côtés et en arrière par sept plaques entourées elles-mêmes par les granulations des tempes et de la nuque. - On compte, de chaque côté de la rostrale, six plaques sus-labiales séparées du cercle orbitaire par une rangée unique de petites écailles; les sous-labiales, arec la mentonnière, sont au nombre de treize. - La quene irrégulièrement verticillée présente, dans toute sa longueur, en dessus et en dessous, des stries formées par les carènes des grandes écailles dont elle est revêtue. - Les cuisses sont granuleuses en arrière, comme les parties supérieures et latérales du tronc. En dedans, les écailles petites, à peine carénées, sont semblables à celles de la face interne des membres antérieurs; les pores fémoraux disposés en une série unique, sont au nombre de douze sur chaque membre. Les écailles de la région externe des quatre pattes, et particulièrement des postérieures, sont grandes et carénées.

I.a teinte générale est un brun verdâtre particulièrement sur la tête et sur la partie antérieure du tronc, dont la moitié postérieure, ainsi que les membres et la queue, est d'un brun tirant sur le rouge. Une raie noire formant collier passe en travers sur le cou; d'autres lignes noires le parcourent d'avant en arrière; la région dorsale et les flancs portent des taches également noires, hautes et étroites; on en voit de semblables sur les membres et sur la queue, où elles forment des demi-anneaux. Les parties inférienres ont une teinte verdâtre légèrement pointillée de noir, et le mâle, déjà caractérisé par la présence de deux grandes écailles concaves situées sous la base de la queue, derrière le cloaque, se distingue, en outre, de la femelle, par une grande tache ventrale bleue, semblable à celle qui se remarque dans le même sexe chez plusieurs Tropidolépides.

Nos deux individus sont à peu près de la même taille; la femelle, qui a

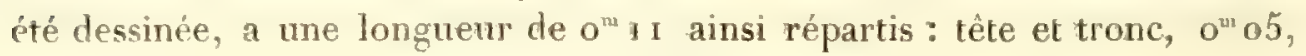


ORDRE DES SAURIENS. - IGUANIENS PLEURODONTES.

queue, $\mathrm{o}^{\mathrm{m}}$ o6. Ils ont été donnés au Muséum par M. Séraphin Braconnier, qui les tenait d'un voyageur revenant du Mexique.

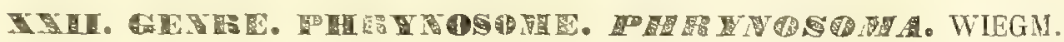

De nombreuses additions ont été faites dans ces dernières années à ce genre remarquable de l'Amérique du nord. Elles sont dues aux travaux des zoologistes des États-Unis, qui ont décrit plusieurs espèces nouvelles, dont l'une mème est assez différente, pour qu'elle ait pu devenir le type d'un genre distinct (Arota Hallowell) 1.

Outre les 3 espèces admises dans l'Erpél. génér. : Phr. de Harlan Wiegm. (Agama cornuta Harl.), Phr. couronné Bl. et Phr. orbiculaire Wiegm, nos collections en ont reçu deux autres, le Phr. téle-plane (planiceps) Hallowell, et le Phr. cle Douglas (Agama Dougl.) Bell, qui sont bien-moins connues, et dont je dois donner la description.

1. Ce genre Anota, fondé par M. Hallowell (Silgreaves expedit. down the Zuni and Culorado rivers, 1833, p. $127, \mathrm{pl} . \mathrm{x}$ ), offre certains caractères essentiels, qui élablissent des différences bien tranchées avec les vrais Phrynosomes, auxquels il ressemble beaucoup par sa conformation générale et par l'armure épineuse do l'occiput. Ces caractères sont les suivants : Tympans cachés; point de piquants sur le dos, qui est lisse; point d'aréte squameuse dentelie sur les flancs. Une seule

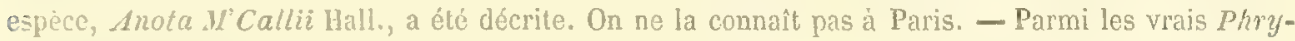
nosomes, dont M. Ch. Girard a donné une interessante monographie (Stansbury's Explor. of the ralley of the great salt lake of Utah, p. 35. et suiv., avec fig.), il y a doux espèces signal'es pour. la premiere fois dans ce travail, et que notre Musée ne possèdo pas: Phrynosoma modestum Girard, p. 365, pl. vi, fig. 4-8, el Phrynos. platyohinos, p. 363, pl. vir, fig. 1-5. Celle dernière se rapproche surtout du Phrynos. de Douglas par le petit développement des épines de l'occiput et par l'uniformité des écailles de la face inférieure de la tête, mais l'examen des figures comparatives de la pl. vir représentant, dans l'une et dans l'autre espèce, le vertex, les écailles épineuses de la région occipitale, les bords de la måchoire inférieure, le profil et la position des narines, montre, comme nous pouvons d'ailleurs nous en assurer sur nos exemplaires du $P$ hr . de Douglas, les différences qui distinguent celui-ci du Phr. platyrhine. Quant au Phr. modeste, pour appprécier les dissemblances qui l'éloignent de ce dernier, avec lequel il a plus d'analogie qu'il ne paraît en avoir avec toul autre, il faut comparer les fig. 4-8 de la pl. vi (Phr. modestum), aux fig. 1-b de la pl. vin (Phr. platyrhinos). - Je dois ajouter qu'il est peut-être permis de supposer avec M. Girard que la figure donnée par M. Holbrook ( N. Amer. herpet., t. II, pl. xus), ne se rapporte pas au $P h r$. orbiculaire décrit dans cet ouvrage. Chez ce dernier, en effet, les épines occipitales et celles du dos sont, en réalité, mains développées qu'elles ne le sont sur le dessin dont il s'agit, et qui n'en montre d'ailleurs que sis à l'occiput, tandis qu'il y en a sur tous nos exemplaires, huit, sans compter le tubercule médian. Enfin, aucune des taches du dos n'a été représentée. 11 est difficile de cire à quel Phrynosome cette planche se rapporterait, mais il y a lieu cependant de supposer que c'est à una espece distincte de celles qui scnt comnez jusqu'i présent. 
XXXVl. - I bis. Phry nosome tête-plane, Phr. planiceps, Hallow.

Idem, Hallow., Proceed. of the Acad. of Philad. Oct. 1852, p. 178. - Id., Id., Sitgreaves Exped. down the Zuni and Colorado rivers, p. 42 i. Rept, pl. vil.

Têle plus déprimée et plus large que celle du Phr. de Harl. ', à épine centrale de l'occiput séparée de celles qui l'croisinent par un large intervalle; écailles ventrales lisses ou à peine carénées; queue plus longue que chez le Phr. de Harl. et moins subitement terminée en pointe; teinte générale jaunâtre et plus claire.

La tête, de volume médiocre, est déprimée, un peu large en arrière; dans sa portion frontale, elle présente, au centre, une dépression assez marquée; le museau est plus obtus que celui de son congénère, chez lequel l'espace compris entre les arcades surciliaires et le bord postérieur de la région du front est plus long et moins large qu'il ne l'est dans l'espèce nouvelle. Celle-ci a une plaque occipitale plus grande et entourée d'un plus grand nombre d'écailles à sommet pointu. L'occiput porte une couronne de neuf épines; elles sont longues, à l'exception de la médiane, qui est un tubercule peu développé. Elles sont disposées de la façon suivante : de chaque còté du tubercule médian, une épine, la plus longue de toutes, puis trois antres se touchant par leur base et moins allongées. Or, tandis que chez le Phr. de Harlan, où la disposition des dents de la conronne est presque la même, c'est la plus postérieure de ces trois épines qui l'emporte sur les deux autres par ses dimensions, c'est au contraire à l'avantage de l'épine médiane de ce petit groupe que celte différence se remarque chez le Phr. téle-plane. Ce dernier a, de plus que le $\mathrm{I}^{\prime} \mathrm{hr}$. de Harl., un rang d'épines pointues, distinctes des écailles sous-orbitaires; elles sont comme la continuation du bord labial inférieur, et sont séparées des épines du bord de la région sous-maxillaire par deux rangs de petites squames. Les écailles épineuses de la mâchoire inférieure sont côtoyées en dedans, sous le menton, et de chaque côk, pai un rang d'écailles plus petites, quoique de même apparence, et dont elles sont à peine éloignées chez le $P$ hr. de Harlan; mais dans la nouvelle espèce,

1. A l'exemple de M. Hallowell, j'emploie pour cette diagnose et pour la description, une forme comparative motivée par l'extrême analogie qui se remarque sous un grand nombre de rapports entre ce $P h r$. et celui de Harlan, dont il diffère cependant, d'une façon très-notable, par certaines particularités, comme le montrent plusieurs des caractères qui lui sont propres, - On trouve dans le travail de M. Hallowell des détails fort complets et très-intéressants sur l'anatomie du Phr. de Harlan. 
l'intervalle est plus considérable et rempli par sept ou huit rangées longitudinales de fines granulations.

Le tronc est couvert, en dessus, d'écailles de forme et de grandeur diverses, ainsi que de tubercules pointus et fortement carénés. La ligne vertébrale est occupée par trois rangs de petites écailles bordées à droite, comme à gauche, par des tubercules peu volumineux et très-serrés. Il y a, sur chaque flanc, une double frange épineuse, et la supérieure est la plus longue.

Après avoir mentionné dans la diagnose les écailles ventrales comme lisses ou comme portant une carène à peine distincte, M. Hallowell se borne à la première de ces deux indications dans les détails descriptifs; aucune saillie, au reste, ne se voit dans celle région sur notre unique spécimen. Chez le Phr. de Harlan, au contraire, ces écailles sont fortement carénées.

Les membres, de longueur médiocre, sont un peu grêles et couverts en dessus d'écailles et de nombreuses épines pointues; celles-ci manquent à leurs faces interne et antérieure où l'on ne voit que des écailles lisses ou carénées; il en est de même à la région inférieure de la queue, dont les côtés et le dessus sont armés de longues épines. Comme M. Hallowell l'a noté pour le mâle qu'il a observé, nous comptons douze pores fémoraux sur l'une des cuisses et onze sur l'autre.

La couleur générale est un jaune clair ou cendré. Les bandes foncées de la g'égion supérieure de la tête sont moins larges que celles qui se voient à la même région chez le $\mu / \varkappa$. de Harlan, dont les grandes taches brunes du. cou sont séparées entre elles par un intervalle plus étroit qu'il ne l'est dans l'espèce dont je présente ici la description; mais pour les taches dorsales, il n'y a aucune différence; on en trouve, au contraire, dans la coloration de l'abdomen, car cette région est à peine maculée dans le Phr. têtte-plane. - Sa taille est semblable à celle de son congénère.

Notre échantillon obtenu de l'Académie de Philadelphie par l'obligeante entremise de M. le docteur Hallowell, a été trouvé près du Rio-Grande dans le Texas occidental où l'espèce paraît ètre assez alıondante. 
XXXVII. - 3 bis, Phry

(Linn. soc. transact., t. XVI, p, 105 , pl. x, sous le nom de Agama Dougl.)

Phr. Dougl., Wagl., Syst. Amph., p. 146. - Id., Wiegm., Herpet. mex., pars I, p. 54. - Ag. Dougl., Harlan, Med. and phys. researches, p. 151.-Phr. Dougl., Holbr., N. Amer. herpet. 1. II, p. 101, pl. xrv. - Id., Gr. Cat. of Lis., p. 227. - Id., Dum., Cat. Rept. Mus. Par., p. 79. - Id., Dum., Répert. Erpét. génér., D. B., t. IX, p. 271.

Écailles ventrales lisses; tẻte courte, triangulaire, pointue, garnie, à sa partie postérieure, de tubercules un peu saillants, mais non de véritables épines; narines ouvertes à l'extrémité antérieure de la créte surciliaire; corps ovale et aplati, couvert en dessus d'écailles et de tubercules peu élevés et peu pointus; dix-huit pores fémoraux de chaque cóté.

Les plaques sus-céphaliques sont polygonales, serrées et imbriquées. I.es tempes et l'occiput sont bordés de neuf petits tubercules peu saillants et dont le médian est tout à fait mousse; il résulte du peu de développement de ces écailles, que le bord postérieur de la tête n’est pas épineux comme dans la plupart des autres espèces. Le Phr. platyrhine Gir. est celui qui lui ressemble le plus sous ce rapport. On compte dix plaques labiales supérieures presque toutes égales entre elles et sept inférieures. Ces plaques sont suivies de quatre tubercules comprimés et pointus, dont le dernier est le plus grand. Le long de chaque branche sous-maxillaire, il règne une série de tubercules petits et lisses en avant, et plus saillants au-dessous de l'angle de la bouche où ils ne rejoignent pas les plaques labiales, dont ils sont séparés par 4 ou 5 rangées de squames granuleuses. - Les écailles des régions supérieures sont lisses, inégales entre elles et rhomboïdales, généralement petites et entremêlées de tubercules triangulaires aigus, moins élevés et moins volumineux que dans les Phryn. de Harlan, couronné et tête-plane; ces tubercules, entourés à leur base par de petites écailles tuberculeuses, forment quatre rangées longitudinales irrégulières, de chaque côté de la ligne médiane, où l'on voit aussi quelques écailles proéminentes. Les flancs ne portent qu'un seul rang de petites épines. En dessous, les écailles sont lisses et polygonales. La queue fort courte, est large et déprimée à sa base, mais elle s'amincit promptement et se termine en pointe; elle ne forme que le tiers environ de la longueur totale.

La teinte générale est, en dessus, un gris clair relevé par des taches foncées transversales. Sur le milieu du dos et dans toute sa longueur, il règne une 
bande d'un blanc jaunâtre; le dessous est d'un blanc d'argent presque uniforme.

Cette espèce n'était pas encore parvenue au Musée de Paris à l'époque de la publication du t. IV de l'Erpét. génér.; mais maintenant, nous en avons deux exemplaires rapportés de Californie par M. Douglas, et donnés par M. le docteur Th. Bell, qui a dit, d'après M. Douglas, que ce Saurien se nourrit d'insectes et de substances végétales, mais les observations de $\mathbf{M}$. Nutall, consignées dans le texte de M. Holbrook, démontrent qu'il est exclusivement herbivore.

- Je ne m'arrêterais pas au $23^{\mathrm{e}}$ genre CaLlisaure fondé par M. de Blainville, d'après un spécimen unique recueilli en Californie par M. Botta, s'il ne paraissait convenable, comme M. Hallowell lui-même l'a supposé, de rapporter à cette division le Saurien qu'il a décrit (Proceed Acad. Philad. Oct. I 852, Captain's Sitgreaves expedit. down the Zuni and Colorado rivers, 1853, p. I16, pl. vi), sous les noms suivants: Homalosaurus ventralis. On ne trouve pas, en effet, dans la description très-soignée du zoologiste américain des caractères suffisants pour motiver une distinction entre ces deux espèces.

- Nul détail n'est à ajouter au $24^{\circ}$ genre Tropidogastre Dum. Bib.

-Dans le ${ }_{2}^{\circ}$ genre, Hicrolophe Dum. Bib., des additions ont été faites. Aucune des espèces nouvellement décrites ne nous est connue, il suffit donc d'en présenter une simple énumération ${ }^{1}$.

- Après les genres Ecphymote Cuv. et Sténocerque D. et B. (26 et 27), les auteurs de l'Erpét. génér. ont placé le genre Strobilure Wiegm. (28) qu'ils ne connaissaient pas. Nous en possédons maintenant trois exemplaires parfaitement semblables à l'espèce unique décrite par Wiegm. (StrobiluTus torquatus). L'un de ces individus encore jeune a été récemment acquis, et les deux autres, qui sont adultes, ont été adressés de Bahia par M. le

1. Steirolepis carinicauda, St. bufonia Fitz. (Syst., p. 73). - St. xanthostigma, St. tigris, St. thoracica, St. quadrivittata Tsch. (Fauna peruana, p. 29 et suiv.). Le genre Microlophe se trouverait ainsi renfermer neuf espèces, si, en outre, avec les denx zoologistes que je viens de citer, on considérait comme distinctes celles qui ont été nommées Tropidurus microlophus Wiegm., Lophyrus araucanus Less. et Garnot, et Stellio peruviana Id., Id., espèces qui, suivant l'opinion émise par Inon père et par Bibron, ne semblent vraiment représenter que des varietés de leur Microlophe de Lessom. Enfin, selon M. Fitz, il faudrait y joindre le Saurien mal connu dont on trouve l'indication dans l'ouvrage de Spix (Lac. bras., p. 13, pl. xvi, fig. 1): Agama semitæniata. 
comte de Castelnau. L'un de ces derniers est très-bien figuré dans la relation de l'Expédit. de ce voyageur dans l'Amérique du sud sous les noms de Doryphorus spinosus Guich.

- Quant au genre Trachycrcle Dum. Bib. (29), nous ne possédons encore que le type unique (Tr. marmoratus) dù à M. D'Orbigny, qui l'a rapporté de Bolivie.

Il n'y a pas d'additions à faire au genre Oplure Cuv. (3o). Des deux espèces qu'il comprend, celle dite $O$. de Maximilien est brésilienne, mais la patrie de l'autre $(O$. de Séba) était restée douteuse pour nous jusqu'à ces dernières années. Nos collections, en effet, ne possédaient qu'un seul spécimen étiqueté, il est vrai, comme provenant du Brésil, mais en l'absence de renseignements positifs à cet égard, on pouvait supposer que cette origine lui avait été attribuée en raison de son analogie parfaite avec le Saurien figuré par Séba (pl. xcvı, fig. 4, t. I) et indiqué ainsi (p. 152 ): Lacerta brasiliensis, Quetz Paleo, cauda annulata et spinosa. On ne peut pas penser cependant que ce reptile vive en Amérique, car nous avons reçu un autre exemplaire de Madagascar par les soins de M. Pervillé ${ }^{1}$.

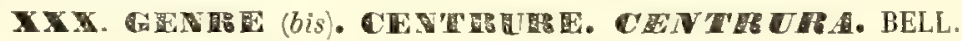

(Foy. of the Beagle, Rept, p. 2o).

Cou et corps sans crête, tronc déprimé, large, à pli longitudinal sur les flancs; queue arrondie, un peu aplatie à sa base, couverte d'écailles grandes, épineuses et verticillées; écailles des régions supérieures très-petites, arrondies, légèrement convexes et lisses; plaque occipitale petite; pas de dents palatines.

Ce genre, comme $\mathrm{M}$ : Bell le fait remarquer avec raison, se rapproche beaucoup des Oplures et des Doryphores de Cuvier, mais surtout des premiers. Les grandes dimensions de la plaque occipitale, l'absence de dents palatines, et principalement la forme plus ou moins aplatie de la queue sont cependant des caractères tout à fait distinctifs du Doryphore. D'autre part les Centrures s'éloignent des Oplures, en ce qu'ils n'ont pas trace de crête sur le

1. Cette anomalie, dans la distribution géographique des Iguaniens pleurodontes, doit être rapprochée de celle que j'ai signalée plus haut en parlant du Brachylophe (p. 527) comme faisant exceplion par son origine australienne au milieu des autres genres de ce groupe, qui sont tous propres au Nouveau-Monde. Il faut, au reste, à ce point de vue, rapprocher du $\mathrm{Br}$. a bandes et de l'Oplure ce Séba, lo Centrure quatre-taches également madécasse, et dont je donne ici la description. 
cou, et que leur écaillure du tronc se compose de squames proportionnellement beaucoup plus petites, et différentes par leur aspect de celles de lopl. de Maximilien, qui sont également sans carènes. La dissemblance est encore plus frappante quand on compare les Centrures à l'Opl. cle Sébr, dont les écailles sont carénées.

\section{XXXviti. - i Centrure flageinetène, Centr. Alagellifer, Bell.}

(Voy. of the Beagle, Rept., p. 25, pl. xir, fig. 2.)

Oplurus Bibronii, Cl. Gay, Hist. de Chile, Rept., décrits par M. Guichenot, p. s̈3, pl. III, fig. 2. Idem, Dum., Cat. Rept. Mus. de Paris, p. 84. - Idem, Id., Répert. Erp. génér., Dun. et Bib., t. IX, p. 274 '.

Pl. xxı, fig. 5, représentant la tète vue en dessus.

Écailles dorsales petites, lisses, convexes et granuleuses; sur toute la téte, un pavé d'écailles granulifornes, presque égales entre elles et semblables à celles du tronc, dont elles ne diffèrent que par leurs climensions plus grandes; celles des tempes, surtout les postérieures, un peu coniques et pointues; bord antérieur de l'oreille faiblement dentelé.

La tête est assez régulièrement triangulaire; la plaque occipitale est trèspeu apparente; le bord antérieur de l'oreille, contrairement à ce qui a été vu par M. Bell, qui n'avait qu'un exemplaire à sa disposition, porte de petites dentelures. La peau du cou forne, en dessous, deux ou trois plis irréguliers, qui remontent sur les côtés de la région cervicale, et le plus inférieur se continue jusqu'aux épaules. 11 y a également un pli le long de chaque

1. Cette espèce est signalée par M. Gray (Cat. of Liz., T. 226) comme synonyme de celle que M. Gravenhorst a nommée Phymaturus palluma (Nova acta Acad. nat. curios., t. XVIII, 2a pars, p. $7 a \mathrm{a} 0, \mathrm{pl} . \mathbf{L V}$, fig. 2). Le zoologiste allemand a cependant fait observer que ce genre Phymature qu'il a établi est une subdivision du genre Urocentron Kaup, ou Doryphore Cuv. C'est ce que démontre d'ailleurs la diagnose de ce nouveau genre, dans laquelle il faut noter l'absence des dents au palais comme dans le Doryphore. Aussi M. Fitzinger, conformément à cette indication, place-t-il dans la synonymie de ce dernier (Syst., ' P. 77) le Phymat., et M. Tschudi, en parlant de l'espèce décrite par M. Gravenhorst, la nomme-t-il Urocentron palluma (Fauna peruana, p. 35). Or, j'ai indiqué plus haut, les caraclères qui distinguent les Centrures, non-seulement des Oplures, mais des Doryphores; je ne puis done pas, arec M. Gray, considérer comme identiques le Centr. flagellif. et le Phymat. palluma. Le Musée de Paris, au reste, ne possède aucun Saurien qui se rapporte à la description de M. Gravenhorst beaucoup plus complète que celle de Molina (Lacerta palluma, Saggio sulla storia nat. del Chili, 1810, p. 189) et que Daudin a reproduite t. IV, p. 46. C'est le vague de ces deux dernières descriptions, qui a motivé le silence des auteurs de l'Erpét. génér., relativement å cet Iguanien à queue épineuse et verticillée. Je dois ajouter que dans une note manuscrite, laissée par Bibron sur son exemplaire de l'ouvrage de Daudin, il a émis la supposition que ce Stellion pelluma du Chili est peut-être le même animal que le Trachycycle marbré Dum. et. Bib.

Archives du Muséum. T. VIII. 
flanc. - Les écailles de la gorge sont semblables à celles du dos, mais sur le ventre, elles sont plus larges et non bombées; elles ne portent pas de carènes.

Les membres sont robustes et couverts d'écailles un peu plus grandes que celles du tronc, légèrement imbriquées, non carénées; celles des faces externe et supérieure des jambes sont coniques.- La queue, dont la longueur dépasse à peine celle du reste du corps, est un peu déprimée à sa base, et cylindrique dans le reste de son étendue; elle est entourée d'écailles verticillées, toutes terminées en une pointe épineuse.

La couleur générale est un brun verdâtre, presque noin sur deux individus. Aucune tache, ni aucune bande ne se remarquent, soit sur le tronc, soit sur la tête; la gorge porte cependant quelques marbrures plus foncées.

Le Muséum possède quatre individus recueillis au Chili par M. Cl. Gay.

Le seul dont la queue soit entière, a une longueur totale de $\mathrm{o}^{\mathrm{m}} 23$ ainsi répartis : tête et tronc, $\mathrm{O}^{\mathrm{ml}} \mathrm{I} \mathrm{O}$, queue, $\mathrm{O}^{\mathrm{m}} 13$.

XXXiX. - 2. Centrure quatre-taches, Centruta quadri-maculatum.

A. Dum. Pl. xxir, fig. $\{$ et $4 a$.

Oplurus quadri-macul. Dum. Bib. N. SS. - Idem, Cat. Rept. Mus. de Par., p. 83. - Id., Répert. Erpét. génér., Dum. et Bib., t. IX, p. 27ł.

Écailles dorsales petites, lisses, légèrement convexes, à peine imbriquées; plaques sus-orbitaires beaucoup moins grandes que les autres plaques sus-céphaliques; écailles temporales à surface très-peu saillante; sur le bord antérieur de l'oreille, cing ou six dentelures assez développées; deux taches rondes d'un noir profond derrière chaque épaule.

La tête forme un triangle, dont le sommet est un peu tronqué, parce que le museau est large et assez obtus. Le contraste entre les petites dimensions des écailles des régions sus-orbitaires et de celles beaucoup plus développées qui recouvrent le reste du crâne, est assez frappant. Il en résulte, ainsi qu'on peut le voir sur les figures $4 a$ et 5 , de notre pl. xxir, une différence importante entre cette espèce et la précédente. Il faut encore noter, comme caractère distinctif, la forme particulière des plaques de la crête surciliaire, qui sont oblongues, un peu obliques de haut en bas et d'arrière en avant, et légèrement imbriquées. - La fig. 4 n'indique pas les plis, mais, outre celui de la région inférieure du cou et dont la prolongation se voit au-dessus de chaque épaule, il y en a un autre le long des flancs, et deux ou trois sur 
les còtés de la région cervicale. - I Les membres sont robustes et couverts d'écailles carénées et imbriquées, plus grandes que celles du tronc.

Isa quene est reproduite dans la plus grande partie de son étendue, sur l'exemplaire du Muséum, mais je trouve dans la description manuscrite de Bibron, faite d'après deux exemplaires observés par lui au Musée de la Sociélé zoologique de Londres, l'indication suivante : "Queue cylindrique, faiblement déprimée à sa base, entourée par des verticilles de grandes écailles quadrilatérales surmontées chacune d'une forte carène, qui les coupe obliquement, de sorte que l'extrémité de cette carène terminée en pointe, aboutit non au milieu du bord postérieur de l'écaille, mais à l'un de ses angles. $)$

En dessus, l'animal est brun; des gonttelettes jaunâtres sont semées sur ce fond, et y forment des lignes interrompues. De chaque côté, derrière l'épaule, on voit deux grandes taches noires, arrondies, placées l'une au devant de l'autre: d'où le nom spécifique de ce Centrure. Le dessus de la tète, des membres et de la queue est d'un brun olivâtre, comme les régions gulaire et sous-maxillaire, qui sont ornées de taches arrondies, jaunâtres; les parties inférieures, dans le reste de leur étendue, ont une teinte claire.

Le spécimen unique de cette espèce a une longueur totale de $0^{\text {nt }} 31$ ainsi répartis : tête et tronc, $\mathrm{O}^{\mathrm{m}} 12$, queue, $\mathrm{O}^{\mathrm{m}} 19$. Il a été rapporté de Madagascar par le colonel Lyoll, et la Société zoologique de Londres en a fait présent à notre Musée.

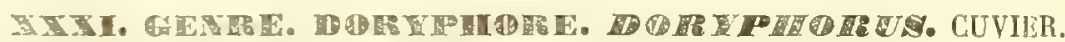

J'ai fait connaître plus haut (note de la page 557 ) les opinions de MM. Fitzinger et Tschudi sur les espèces qu'ils pensent devoir rapporter au genre dont il s'agit. Je n'ai pas à revenir sur ce sujet, je dois seulement dire que nul échantillon nouveau n'ayant été reçu au Musée de Paris depuis 1837 , à l'exception de celui qui est décrit plus Join, la même incertitude nous reste relativement à la possibilité de distinguer les deux espèces signalées par Daudin. Celle que M. Gravenhorst a nommée Phymaturus palluma nous est inconnue. 


\section{XL. - 2. Doryphore tête-Jadne, Doryph. flaviceps, Guichenot.}

Explorat. Amér. mérid., comte de Castelnau, Rept., p. 26, pl. III, fig. 2.

Doryph. azuré (variété noire sans bandes transversales), Dum., Cat. Rept. Mus. de Par., p. 85 '.

Plaques sus-céphaliques bombées et un peu saillantes à leur centre, légèrement rugueuses; quelques plaques sus-orbitaires plus grandes que les squames environnantes; écailles dorsales peu carénées; queue aplatie, large, terminée en pointe, à écailles également carénées, disposées en verticilles; tête d'un brun jaunätre clair, et le reste du corps d'une teinte sombre uniforme.

Le dessus du crâne est plat, et le museau arqué et déclive en avant. Toute l'écaillure sus-céphalique porte des rugosités produites par des inégalités de leur surface, qui donnent à ces squames un aspect analogue à celui des peaux de inaroquin chagriné. La plaque occipitale est assez grande et polygonale. On voit à l'extrémité postérieure de la région sus-orbitaire, trois ou quatre plaques dilatées en travers, et plus grandes que celles qui les précèdent et les entourent. On compte is plaques sus-labiales en y comprenant la rostrale, qui est plus longue que haute, et I 2 sous-labiales avec une grande mentonnière. - Les écailles sur la nuque sont un peu pointues et moins grandes que sur le dos. - Celles de la gorge sont entourées par de très-petits tubercules, qui manquent à la région abdominale, où les écailles présentent une faible saillie médiane.

L'aplatissement de la queue a probablement été angmenté par la dessiccation; elle est comparable par sa forme à une feuille allongée et pointue, comme celle du laurier par exemple. Ses écailles, de médiocre grandeur, sont disposées en bandes transversales, régulières et surmontées de carènes.

Les membres sont assez robustes, et sur leurs régions externe et postérieure, les écailles sont carénées.

1. C'est avec doute que j'inscris ce Saurien parmi les Doryphores; car si, par le plus grand nombre de ses caractères, il doit prendre rang dans ce genre, il présente cependant quelques particularités qui semblent l'en éloigner; ainsi sa queue est plus aplatie et plus allongée, et ses écailles portent de petites carènes formant, par leur réunion, des lignes obliques dirigées d'arrière en avant et de bas en haut vers la ligne médiane du dos. Néanmoins, le mauvais état de conservation de notre unique spécimen, qui a été longtemps desséché avant d'être placé dans l'alcool, ne permettant pas de donner une détermination suffisamment précise, je crois convenable de le laisser auprès des Iguaniens auxquels il ressemble le plus, en attendant la possibilité d'une comparaison ultérieure avec des individus dont les téguments soient moins altérés. 
La couleur générale de cet Iguanien est très-sombre et présente un contraste frappant avec la teinte beaucoup plus claire de la tête, qui est d'un brun jaunâtre, irrégulièrement pointillé de noir. Une bande jaune et étroite traverse la nuque.-L'individu unique, type de cette espèce nouvelle, donné au Muséum par MM. de Castelnau et Deville, provient de la mission de Sarayacu (Pérou). Il est long de $\mathrm{o}^{\mathrm{m}} 20$ (tête et tronc, $\mathrm{o}^{\mathrm{m}} 1 \mathrm{I}$, queue, $\mathrm{o}^{\mathrm{m}} \mathrm{og}$ ).

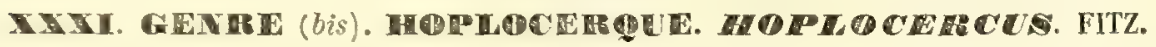

Tête triangulaire, légèrement aplatie, couverte de granulations polygonales et à plaque occipitale très-petite; cou el dos sans crête; écaillure du dos entremêlée de grandes écailles carénées et luberculeuses; des dents au palais; queue épaisse, fort courte, non verlicillée, plate, et dont la région supérieure porte des écailles épineuses, qui sont très-saillantes sur les rangées médiane et latérales ${ }^{1}$.

Les caractères indiqués dans cette diagnose montrent les analogies, et en même temps les différences qui se remarquent entre ce genre et les Oplures, les Centrures on les Doryphares. Chez ces derniers, en effet, la queue n'est ni aussi courte, ni aussi trapne; leur plaque occipitale est plus grande, et ils manquent de dents au palais. Dans les deux autres genres, la queue est cylindrique et nom aplatie. Enfin, chez aucun des Sauriens auxquels je compare $l$ Hoplocerque, les écailles du tronc n'offrent une semblable diversité de forme et de grandenr.

4. Le type de ce genre, reçu depuis deux ans environ au Musée de Paris, a été signalé dans la Revue de $z 00$ l., 18\%\{, p. 239, sous le nom de Pachycerque aiguillonné (Pachycercus aculeatus), qui lui avait élé donné par MA. Alfr. Dugès et $\mathrm{S}$. Braconnier. J’ai, moi-mème, reproduit leur description, à la fin d'un Mémoire publié dans ce mème recueil (1834, p. 544), ayant pour titre : Essai d'applicat. a la classe des Rept. d'une distribut. en séries parallèles, et accompagné d'une planche représentant l'animal entier, ainsi que des détails amplifiés. Dans une note altérieurement insérée dans la

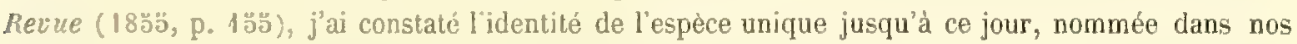
collections Pachycercus aculeatus, et de celle qui est étiquetce à Vienne, en Autriche, par les soins de If. Fitz. : Hoplocercus spinosus. Ce naturaliste la place, dans son Syst, entre les genres Doryphore et Urocentre, parmi les Doryphoriens, dont il forme une famille distincte, (voy. plus haut, p. 507). - Je dois faire remarquer ici que les Jguaniens pleurodontes à écaillure hétérogène sont peu nombreux. Les plus remarquables, sous ce rapport, sont les Phrynosomes, puis l'Anolis (Acantholis) loysiana Coct. Le Phymatolépide A. Dum. que j'ai décrit p. 548, porte des tubercules. Chez les Corytophanes, on voit des écailles carénées plus grandes que celles qui les environnent. Enfin, les Anolis caméléonide (Chamseleolis) Coct. et héléroderme A. Dum, ont de grandes écailles plates enlourées par de petites squames. 


\section{XLI. - I. Hoplocerque Épinedx, Hopl. spinosus. Fitz., Systema, p. 78.}

Pachycercus aculeatus A. Dugès et Sér. Braconnier, Revue de $\approx 00 l ., 1854$, p. 239 ef 544, pl. XII.

Régions supérieures d'une teinte faure grisätre; tête brune; sur le dos, sỉx bandes transversales également brunes; sur les épaules, une bande blanchätre; sur les flancs et sur les membres, des taches d'un brun noirâtre; rentre unicolore.

La tête est aplatie en arrière, jusque sur la partie antérieure des paupières; là, elle s'abaisse vers le museau, en formant une courbe légère. Le tronc assez court et aplati porte, sur chaque flanc, des plis longitudinaux, dont le supérieur commence derrière le tympan et passe au-dessus de l'épaule. La queue presque plane en dessus, convexe sur les bords, et large, forme un triangle à côtés arrondis. De l'ensemble de cette conformation, il résulte que le Pachycerque est un animal peu élancé. Ses membres, sont assez courts, car les postérieurs relevés le long du tronc n'arrivent pas à l'aisselle, et les antérieurs placés dans le même sens ne s'étendent pas au delà de l'oil.

Le dos présente, au milieu, des granulations du fond, une multitude de petites plaques carénées, d'autant plus volumineuses, qu'elles sont plus rapprochées de la queue, et formant des stries longitudinales et transversales irrégulières. Sur la queue, les grandes écailles à carène prennent davantage l'apparence de tubercules, car celles des rangées médiane et latérales sont surmontées d'une sorte de petite protubérance crochue, d'où résultent trois crêtes tranchantes formées chacune par une douzaine de fortes épines courbées en arrière, et les crêtes latérales dépassent en hauteur celle du milieu. - Les écailles situées au-dessus des yeux sont toutes égales entre elles et à celles qui recouvrent les autres points du crâne, à l'exception des plaques disposées en demi-cercle formant la limite en avant, en arrière et en dedans, des régions sus-orbitaires, car elles l'emportent sur toutes les autres par leurs dimensions. On compte 2 I plaques à la lèvre supérieure en y comprenant la rostrale; elles sont plus petites que les inférieures dont il y a onze paires séparées par une mentonnière assez grande.

La longueur totale de notre unique échantillon qui provient de la province de Saint-Paul (Brésil) est de on 098 (jtète et tronc, $o^{\text {m }} 069$, queue, $\mathrm{o}^{\mathrm{m}} 029$ ). 
DEUXIÈME SOUS-FAMILLE DES IGUANIENS : LES ACRODONTES.

Le caractère général et essentiel de ces reptiles est fourni par la disposition du système dentaire. Leurs dents, en effet, au lieu d'être logées dans un sillon creusé sur la face interne des mâchoires, comme celle des espèces appartenant à la sous-famille que je viens de passer en revue, sont solidement fixées sur le bord saillant et plein des mâchoires, dont elles occupent, en quelque sorte, le sommet. Ce sont ces différences que Wagler a voulu rappeler en se servant, pour ces deux groupes, des mots Pleurodontes et Acrodontes. Elles ont, au reste, une grande importance, car elles se lient à d'autres particularités remarquables. Ainsi, tous les Acrodontes sont privés de dents palatines, tandis qu'il y en a chez le plus grand nombre des Pleurodontes, et tous sont originaires de l'Ancien-Monde. - Les Agames proprement dits, constituant un type bien caractérisé de ces Sauriens, on les nomme souvent Agamiens ou Agamides, par opposition aux Iguanides ou 1guaniens. Ces derniers sont plus nombreux, non-seulement comme espèces, mais comme genres: d'où jl résulte que j'ai eu beaucoup plus d'additions à faire, qu'il ne m'en reste maintenant à présenter sur les Acrodontes, qui ne comprennent que I 7 genres, tandis qu'on peut en distinguer au moins $4 \mathrm{I}$ dans l'autre sous-famille, savoir : 3 I genres admis dans l'Erpét. génér. et dix nouveaux, dont j'ai exposé l'histoire dans les pages qui précèdent où j'ai décrit 4 I espèces nouvelles ou peu connues.

- Je n'ai aucun détail nouveau à donner sur le genre Istiure $\left(3_{2}\right)$, mais il n'en est pas de même pour le suivant.

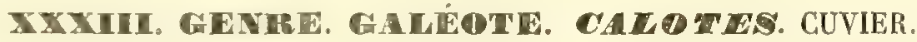

Ce genre, très-naturel, établi par Cuvier, a été avec raison partagé en deux groupes secondaires par M. Kaup, d'après l'arrangement des écailles. Chez certaines espèces, en effet, elles sont disposées de telle façon qu'elles forment des stries obliquement dirigées soit d'arrière en avant et de haut en bas, comme dans les Bronchocèles (voy. notre pl. xxıv, fig. 4), soit également d'arrière en avant, mais de bas en haut : tels sont les Galéotes proprement dits (pl. xxrv, fig. 5). Une troisième disposition, enfin, se présente; elle est propre aux espèces réunies par M. Gray en un genre Salea, auquel il faut réunir le groupe que j’ai nommé Mécolépide (Cat. Rept. Mus. de 
Prr., p. 87, de $\mu$ ñ̃ros, longueur, et $\lambda \varepsilon \pi t \varsigma$, écaille), par opposition à ce qui se voit dans les deux autres sous-genres, car ici, les écailles sont dirigées, sans aucune obliquité, d'avant en arrière ( $\mathrm{pl}$. xxıv, fig. 1, 2, 3 et I $a$ ). - Cette dernière division est la seule dont j'aie à m'occuper, le Musée de Paris n'ayant reçu aucun Galéote ou Bronchocèle qui puisse être rapporté à quelque espèce nouvelle ${ }^{1}$.

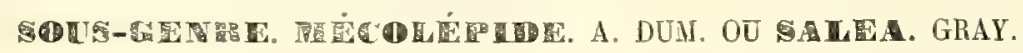

Écailles du tronc formant des bandes longitudinales et parallèles entre elles et à la ligne médiane, de sorte que leur extrémité libre est tournée directement en arrière.

XIII. - i Mécoléplde tri-ÉPineux, Mecolepis trispinosus, A. Dum.

P1. xxip, fig. 4. -Idem, Id., Cat. Rept. Mus. de Paris, p. 88.

Depuis la nuque, jusqu'à la base de la queue, une crête formée par trois rangs parallèles l'épines, dont le médian est beaucoup plus développé que les deux latéraux, très-basse au-dessus des épaules, et se continuant en un seul rang sur le premier tiers de la queue, dont les deux autres tiers ne portent qu'une petite carène; de fines bandes noires longitudinales sur le dos et sur les flancs.

1. Ces espèces, au reste, sont nombreuses. - I. Aux trois Bronchocèles décrits dans l'Erpét. génér., il faut ajouter : $1^{\circ}$ celui que M. Berthold a fait connaitre et figurer (Ueber verschiedene neue oder seltene Amphibienarten in Abhandl. der Konigl. gesellschaft der wissensch. zü Gottingen, I, 1838-41 (1843), p. 59, pl. 1I, fig. 6): Bronchocela intermedia; $2^{\circ}$ une espèce signalée par M. Gray (Cat. of Liz., P. 241), comme étant nommée au Musée de Leyde par M. Schlegel, Br. celebensis.

II. Dans le sous-genre Galéote, on compte maintenant douze espèces, au lieu de quatre seulement que notre Musée possède, et parmi lesquelles il s'en trouve deux, qui ont été décrites pour la première fois par mon père et par Bibron en 1837 : Caloles Rouxii et $C$. mystaceus. Celles-ci ont été de la part Le M. Blyth, l'objet de nouvelles descriptions, ainsi que les G. versicolore et ophiomaque, plus anciennement connus, et que trois espèces nouvelles (Notices and descript. of various Rept. new or litlle known in Journ. of the asiatic soc. of Bengal, 4853, no vir, p. 647), nommées par ce zoologiste: $C$. gigas, platyceps et tricarinatus. Il présente, en outre, des détails sur deux autres Galéotes: C. Emma et C. viridis Gray. Il faut y joindre, d'après le $C a t$. du Musée britannique, C. Maria et C. minor Gr. Enfin, la douzième espèce est C. nemoricola Jerdon (Cat. of Rept. inhabit. the Peninsula of India in Journ. of the Asiatic Soc. of Bengal, 1853, $\mathrm{n}^{\circ} \mathrm{v}, \mathrm{p} .171$ ).

III. Aucun des trois Mécolépides décrits ici ne se rapporte aux Sauriens nommés par M. Gray: Salea Horsfieldii (Cat. of Liz., p. 242), S. Jerdoni (Annals of nat. hist., $2^{\mathrm{e}}$ serie, t. XVIII. p. 239), ni à l'espèce que $\mathbf{H}$. Blyth (Curator. As. Soc.) fait connaitre (loc. cit., p. 473, note): $\therefore$ gularis. 
La tête a beaucoup d'analogie avec celle des Galéotes par sa conformation générale, et par un léger renflement qui se remarque, de chaque côté, derrière l'articulation de la mâchoire. La région sus-céphalique un peu creuse dans son milieu, particulièrement entre les saillies sus-orbitaires, est recouverte dans toute sa portion antérieure, depuis le museau jusqu'au delà des yeux, de plaques légèrement bosselées, et de dimensions médincres. Les sus-oculaires sont moins grandes; celles de la partie postérieure de la tête sont encore plus petites, et l'on distingue à peine la plaque occipitale qui, par sa forme, diffère peu des écailles voisines, mais elle est facilement reconnaissable au caractère habituel tiré de la présence, dans son point central, d'un petit espace circulaire, où l'épiderme offre un autre aspect que partout ailleurs. - Au-dessus des plaques sus-labiales, dont on compte sept de chaque côté, séparées par une rostrale allongée, mais basse, on voit une rangée de grandes écailles; il y a 17 sous-labiales, en comprenant dans ce nombre la mentonnière.

Toutes les écailles du tronc, de la queue et des membres sont grandes et carénées. De chaque côté de la nuque, on en trouve une plus saillante que les autres et située au nivean de l'origine de la crête médiane, dont les trois rangs d'écailles épineuses sont très-rapprochés les uns des autres.

La queue, comprimée à sa base et dans une grande partie de son étendue, devient cylindrique vers la pointe; elle est longue et très-effilés.

La couleur générale est, en dessus, un brun fauve relevé par de fines rayures noires parallèles, tracées, d'avant en arrière, sur le milieu de chaque rangée longitudinale d'écailles. Les plus longues épines de la crête dorsale sont également ornées de petites taches noires. On en voit de semblables sur Ja tête. Du bord inférieur de l'oeil, il part une bande formée par de petites lignes de la même teinte et qui, traversant le tympan, se dirige en bas et en arrière, vers l'épaule, pour se continuer sur la face externe du membre antérieur où, par suite d'interruptions régulières, elle représente une suite de demi-anneaux. Les membres postérieurs offrent le même aspect, et la queue est annelée dans toute sa longueur, mais avec irrégularité. Les régions inférieures ont une teinte plus claire que le dos et les flancs.

La longueur totale de notre unique spécimen est de $\mathrm{o}^{\mathrm{m}} 29$ ainsi répartis : tête et tronc, $\mathrm{o}^{\mathrm{m}}$ og; queue, $\mathrm{o}^{\mathrm{m}} 20$. Il a été pris aux Indes orientales, sur les monts Nilgherry, par M. Perrotet, qui en a fait présent au Muséum. 
XLIII. - MÉCOLÉPIDE HÉRIssÉ Mecolepïs hïrsutus, A. Dum.

Pl. xxry, fig. 2.

Idem, Id., Cat. Rept. Mus. de Paris, p. 88.

Depuis la nuque, jusqu'à la base de la queue, une crête assez élevée, formée par un seul rang d'épines fortes et espacées, mais très-basse au-dessus des épaules; des bandes noires transversales sur le dos.

La disposition de la crête formée par un seul rang d'épines est un impor. tant caractère distinctif de cette espèce, qui diffère, en outre, de la précédente, par la forme un peu plus effilée du museau; la tête, d'ailleurs, a, dans sa conformation générale, les plus grands rapports avec celle du M. triépineux, dont la plaque occipitale est beaucoup plus petite qu'elle ne l'est ici où les plaques du voisinage sont également plus grandes. Au nombre de ces dernières, et parmi les postérieures, il y en a quatre, d'apparence tuber. culeuse, situées par paire, de chaque côté de l'origine de la crête. - Les plaques sus-oculaires, et particulièrement les plus externes, sont moins développées que celles qui recouvrent le reste du crâne; toutes ces squames sont, comme dans l'espèce précédente, un peu rugueuses et faiblement carénées. On compte, à chaque lèvre, I 7 plaques poreuses, en y comprenant la rostrale et la mentonnière; elles ne diffèrent pas de celles de l'autre espèce; il en est de même pour le rang d'écailles placées au-dessus de la lèvre supérieure.

Toute l'écaillure du tronc, des membres et de la queue est carénée; cette dernière cependant l'est à peine à sa base; elle est un peu comprimée dans toute son étendue, à l'exception de sa pointe où elle est cylindrique et effilée.

La teinte générale est brun-fauve, comme chez le Ml. tri-épineux, mais au lieu de raies longitudinales noires, on ne voit ici que des bandes transversales de la même nuance, au nombre de six ou sept, et qui représentent des demi-anneaux sur la queue et sur les pattes. Une tache temporale s'étend, de même que dans l'autre espèce, depuis l'angle postérieur de l'oil, jusque sur l'épaule. Quelques lignes noires descendent obliquement, d'avant en arrière, de la lèvre inférieure sur le cou, dont la peau ne forme qu'un petit fanon très-peu développé. Les régions inférieures sont d'un brun jaunâtre clair et unicolore. 
Le Musée de Paris possède deux individus parfaitement semblables entre eux, donnés par la Société zoologique de Londres qui les avait reçus du Bengale.

La longueur totale du. plus grand est $\mathrm{O}^{\mathrm{m}} 248$ ainsi répartis : tête et tronc, $0^{m}$ o-8; quene, o 170 .

XliV. - 3. Mécolépide sillonns, Mecolepis sulcalus, A. Dum.

Pl. xxiv, fig. 3.

Idem, Id., Cat. Rept. Mus. de Paris, p. 8.9.

Sur la nuque, depuis l'occiput jusqu'au-dessus des épaules, une petite crette peu développée, formée par deux rangs d'épines très-rapprochées; depuis les épaulcs, sur la ligne moyenne du tronc et des deux premiers tiers de la quene, un sillon étroit, très-peu profond, résultant de ce qu'un petit intervalle sépare l'un de l'autre les deux rangs médians d'écailles, dont les carènes sont plus saillantes que partout ailleurs, et forment ainsi une inuble créte peu élevée; sur le dlos, des aches noires disposées en bandes transversales courtes et irrégulières.

Les caractères qui viennent d'être indiqués, et surtout la disposition des écailles de la ligne dorsale, ne permettent aucune confusion avec les deux autres espèces; celle-ci offre, en outre; cette particularité que la tête est proportionnellement plus courte, et le museau plus mousse et plus déclive. Ies écailles des flancs sont manifestement plus grandes que celles du dos. Toutes les pièces de l'écaillure sont carénées. Tues plaques de la tête offrent une grande analogie avec celle des deux Mécolépides précédemment décrits. Elles sont moins rugueuses et moins fortement carénées chez l'un de nos individus adultes que chez l'autre, où ces rugosités d'ailleurs, ne sont pas aussi prononcées que dans les deux espèces précédentes. La plaque occipitale, comme clez le M. hérissé, dépasse, par ses dimensions, celle du M. tri-épineux. - La queue, comprimée à sa base, s'arrondit ensuite et se termine en une pointe effilée.

La conleur générale est un brun plus foncé sur le dos que sur les parties latérales, dont la nuance est verdâtre " excepté dans les points où cette teinte plus sombre se prolonge sous forme de taches irrégulières plus apparentes chez l'individu adulte représenté pl. xxiv, fig. 3, que chez un autre également adulte, qui est aussi conservé dans nos collections. Comme dans les espèces précédentes, la région temporale est parcourue, d'avant en arrière, par une large raie foncée, qui se porte jusque sur l'épanle, 
et les membres, ainsi que la queue, sont irrégulièrement annelés de brun. - Les jeunes sujets sont d'une teinte plus uniforme et plus claire.

Longueur totale du plus grand spécimen, $\mathrm{O}^{\mathrm{m}}{ }_{2} 6$ ainsi répartis : tête et tronc, $\mathrm{O}^{\mathrm{m}} \mathrm{0} 0 \mathrm{0}$; queue, $\mathrm{o}^{\mathrm{m}} 190$. - Cette espèce est due à M. Perrotet; elle habite les monts Nilgherry (Indes-orientales).

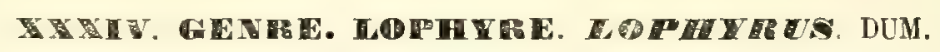

(Zool. analyt., p. 80.)

XLV. - 2 bis. Lophyre spinipède, Loph. spinipes, A. Dum.

(Cat. Rept. Mus. Par., p. 90)'.

Idem, Id., Répert. Erpét. geenér. Dum، Bib., t. IX, p. 276.

Bords surciliaires, à peine anguleux, sans épine à leur extrémité postérieure; pas d'écailles épineuses sur la nuque; une crele cervicale non prolongée sur le dos, qui, comme la première moitié de la queue, ne porte qu'une petite carène dentelée; sur les membres, mais plus partículièrement sur les jambes, des écailles plus grandes que les autres, régulièrement disposées en rangées obligues et munies d'une forte carène.

Par tout l'ensemble de sa conformation, ce Loph. se rapproche beaucoup du Loph. armé, dont il se distingue facilement par la saillie moins prononcée de la carène des squames ventrales, par l'absence d'une écaille épineuse à l'extrémité postérieure de l'arcade surciliaire, et d'un faisceau d'épines de chaque còté de la nuque, où l'on ne voit qu'un petit nombre de tubercules mousses et épars, de même que sur le dos et sur les flancs. Les membres semblent être épineux, tant la carène qui surmonte les grandes

1. Ce genre, que mon fère a établi d'après l'espèce nommée par Séba Lacerta tigrina pectinata, est maintenant divisé par (ifférents zoologistes en plusieurs genres et sous-genres, mais il forme un groupe très-naturel auquel il convient de laisser l'ancienne dénomination. Il comprend aujourd'hui neuf espèces bien distinctes. On peut les partager en deux groupes secondaires suivant la disposition de !a crète: I. Espèces à crête sur le cou seulement. $1^{\circ} \mathrm{L}$. tigré Dum., $2^{\circ} \mathrm{L}$. de Kuhl, Boie, qui gểnéră. lement confondu avec le précédent, en est cependant distinct, comme l'a étubli M. Schlegel dans un intéressant Mémoire sur ces Rept. (Arch. de la Soc. Natura artis magistra, $3^{\mathrm{e}}$ livr. 18ä4, p. 4). Une représentation coloriée du màle et de la femelle est jointe à ce travail, ainsi qu'une figure du $L$. tigré, et ces trois dessins sont faits d'après le vivant; $3^{\circ} L$. armé Dum. Bib. (Agama armata $\mathrm{Gr}$ ); $4^{\circ}$ L. spinipède A. Dum. - II. Espèces à crete nuchale et dorsale. A, interrompue audessus des épaules. $5^{\circ} \mathrm{L}$. dilophe Dum. Bib.; $6^{\circ} \mathrm{L}$. épineu $x$ Hombr. et Jacquinot; B, non interrompue; 7" L. de Bell Dum. Bib.; $8^{\circ}$ L. de Sumatra Schl. (loc. cit.); $9^{\circ}$ L. de Bornéo Id. (Id.); ces deux derniers sont inconnus au Musée de Paris. 
écailles entremêlées aux plus petites est saillante. - Quand on compare cette nouvelle espèce au Loph. tigré qui, avant l'état adulte, porte aussi sur les membres de grandes écailles carénées, on voit des différences si tranchées dans l'aspect, chez ce dernier, de la région postérieure du crâne, par suite de la forme très-manifestement anguleuse des arcades surciliaires et de la disposition des grandes écailles de la base de la crête, que nulle confusion n'est possible. - Il n'y a, sous la gorge, qu'un très-petit fanon. La membrane du tympan est encore plus épaisse que chez le Loph. armé, et ne se distingue des téguments environnants que parce qu'elle ne porte point d'écailles à son centre.

La teinte générale est un brun moins foncé en dessous qu'il ne l'est en dessus. Sur la nuque et sur les épaules, la coloration est plus claire. On ne distingue que confusément, sur la ligne médiane, des taches transversales brunes très-foncées. A la base de la queue, et à la région supérieure, il y a, de chaque côté, une tache noire. La queue, dont les écailles inférieures portent de fortes carènes qui, par leur réunion, forment des stries longitudinales saillantes, est irrégulièrement annelée de brun noirâtre; des bandes semblables ornent les membres.

La longueur totale du spécimen type de cette espèce est de $0^{\mathrm{m}} 34$ ainsi répartis: tète et tronc, $\mathrm{o}^{\mathrm{m}} 1 \mathrm{I}$; queue, $\mathrm{o}^{\mathrm{m}} \mathrm{2}_{2} 3$. 11 a été rapporté de la NouvelleHollande par M. J. Verreaux.

XIVI. - 3 bis. Lophrre Ĺpineux, Lophyrus spinosus, Hombr. et Jacq.

(Toy. au pole sud et dans l'Océanie, sur les corvettes l'Astrolabe et la Zélée, Rept., pl, III, sans texte.)

Idem, Id., Id., Cat. Rept. Hus. Paris, p. 91, où la description a élé donnée pour la première fois. - Id., Id, Répert. Erpél génér., Dum. Bib., t. IX, p. 276. Bronchocela marmorata Gr., Cat. of Liz. of the british Mus., p. 242.

Bord surciliaire curviligne, sans épine à son extrémité postérieure; point de tubercules sur l'occiput, ni de faisceaux d'épines sur la nuque; sur le cou, une créte formée par un seul rang d'écailles épineuses médiocrement longues, mais plus haute que sur le dos où, après une pelite interruption au-dessus des épaules, celte crcle se continue, en diminuant graduellement de hauteur, jusqu'a la base de la queue, dont le bord supérieur porte, dans son premier tiers, une carène dentelée.

D'après la forme un peu allongée de sa tête, mais surtont d'après la disposition de sa crête, ce Loph. se rapproche surtout de l'espèce décrite pour 
la première fois par mon père et par Bibron, et qu'ils ont nommée $L$. dilophe, mais la confusion est impossible, car, contrairement à ce qui s'observe chez ce dernier :- ${ }^{\circ}{ }^{\circ}$ le fanon n'a pas de dentelures à son bord libre, ni de tubercules épars sur ses faces latérales; $2^{\circ} 1^{\prime}$ écaillure du tronc est homogène; $3^{\circ}$ enfin, la crête ne se prolonge pas sur la queue, dont le bord supérieur ne porte qu'une carène fortement dentelée. Cette queue est d'ailleurs fort allongée et très-effilée. - La membrane du tympan est bien visible; elle est placée à la partie antérieure d'un espace elliptique granuleux, long de $\mathrm{o}^{\text {Ш }} \mathrm{O}_{2}$ environ, large de $\mathrm{o}^{\mathrm{n}} \mathrm{OI}$, et bordée par un double rang de grandes écailles.

La teinte générale est un brun jaunâtre en dessous, rougeâtre sur le dos et sur les flancs; les parties latérales de la tête et du cou sont presque d'un rouge brique, de même que les larges anneaux qui entourent la queue en alternant avec d'autres anneaux bruns, irréguliers comme eux. Les doigts et le bord libre du fanon sont nuancés de vert jaunâtre.

L'individu unique, type de cette espèce, a été rapporté de Samboangan (île Mindanao, Archipel des Philippines), par MV. Hombron et Jacquinot.

Sa longueur totale est de $\mathrm{o}^{\mathrm{m}} 565$ ainsi répartis : tête et tronc, $\mathrm{a}^{\mathrm{m}} 14 \mathrm{o}$, quene, $\mathrm{o}^{\mathrm{m}} 425$.

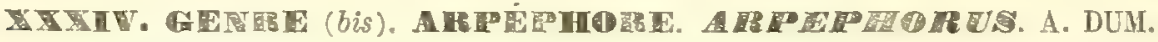

(Cat. Rept. Mus. Par., p. 92, et Revue de $\approx 00 l ., 1851$, p. 243, pl. vir ".)

Museau terminé par un prolongement membraneux, comprimé, mince, plus long que la têle, en forme de sabre ou de faux à deux tranchants: l'un supérieur, légèrement concave, l'autre inférieur convexe; moins large à son extrémité libre, qui se relève en pointe, qu'il ne l'est à sa base, où il est entouré par quelques grandes écailles molles; queue longue et comprimée, surmontée, dans toute son étendue, d'une crête qui est moins haute sur le dos el sur le cou; tympan petil, mais apparent.

De l'extrémité antérieure de la tête, il part un prolongement falciforme, qui est mince, membraneux et non recouvert d'écailles. Sa base est entourée,

1. De «ँ comme exprimant le mieux possible le caractère singulier et tout à fait exceptionnel du Reptile qu'il sert à désigner, sa terminaison masculine le distinguant d'ailleurs, et d'une façon suffsante, du mot 
comme une corolle dans son calice, par quatre écailles : la supérieure et l'inférieure sont pliées sur elles-mêmes et la reçoivent dans l'ścartement de leurs deux lames qui, s'appliquant sur les faces latérales, y rejoignent, par leurs bords, une large écaille située de chaque côté. Derrière l'écaille supérieure, il y en a trois petites également anguleuses, dont le sommet assez aigu est tourné en haut. Elles sont suivies par une grande plaque triangulaire à sommet antérieur, et appliquée par sa base sur la ligne médiane.

XlVII. - 1. Arpéphore trois-bandes, Arpeph.tri-cinctus, A. Dum.

Teinte gènérale brune; sur le dos, trois larges bandes transversales d'un jaune vif.

Par suite de la dessiccation à laquelle ce Reptile avait été soumis avant d'ètre plongé dans l'alcool, car on le trouva piqué dans une boite parmi des insectes, sa conformation générale a été un peu modifiée; on voit cependant qu'elle se rapproche de celle des Lophyres ou des Istiures, auprès desquels Bibron avait provisoirement placé cette espèce. - Les écailles de la tète sont toutes un peu rugueures; il n'y a pas de carènes sur celles du dos, mais on en voit de très-manifestes sur les membres, principalement à leur face interne, sur toute la queue, dont le bord inférieur porte un double rang de petites épines, et enfin sur les régions inférieures du tronc, si ce n'est sous la gorge, qui est recouverte d'écailles un peu tuberculeuses.

Des trois bandes jaunes transversales du tronc, la première, qui occupe la région sus-scapulaire, est la plus étroite et la moins longue; les deux autres ont une largeur de $o^{\text {m }}$ or environ et descendent sur les flancs et sur le ventre où elles se terminent, sans se rejoindre par leurs extrémités.

Le type unique de ce genre nouveau provient de Java. Sa longueur totale,

Harpephora employé par G. Fischer de Waldheim (Index Orthopterorum Sociefati traditorum in Bullet. Soc.impér. natur. Moscou, 1846, t. XIX, ze partie, p. 479), pour un genre nouveau de la fam. des Locustina et comprenant deux espèces. - Dans la note que j'ai consacréo. (Revue de zool.) à la description de ce bizarre Agamien, j'ai rappelé la plupart des exemples connus de reptiles à prolongement membraneux des sourcils ou du museau, mais dont les plus curieux, sous ce rapport, sont les Xiphorhynques ou grands serpents d'arbre de Madagascar dits Langaha, et nommés spécifiquement l’un, ensifera, et l'autre, crista-galli (Erpét. génér., t. VIII, $2^{\circledR}$ partie, p. 802). - Il est à peine nécessaire de rappeler que ce lézard ne peut ètre confondu avec aucun autre, en raison de sa confor mation toute spéciale, qui motive son classement auprès des genres Cératophore et Lyriocéphale. dont la proéminence nasale offre une légère analogie avec celle de l'Arpéphore. 
y compris le prolongement falciforme du museau, est de $0^{\mathrm{m}} \mathrm{I} 68$, ainsi répartis : tête, $\mathrm{o}^{\mathrm{m}}$ or 9 ; son prolongement, $\mathrm{o}^{\mathrm{m}} \mathrm{o} \mathrm{or}$; tronc, $\mathrm{o}^{\mathrm{m}} \mathrm{o} 45$; queue, $\mathrm{o}^{\mathrm{m}} \mathrm{o} 83$.

- Je n'ai aucun détail nouveau à donner sur les genres 35 à 39 , dont deux nous manquent encore (Отосrypte, Wiegm. et Cératophore, Gr.). Le troisième (LyRıcépHALE) n'est, jusqu'à ce jour, représenté que par un échantillon unique au Musée de Paris. - Quant au Sitane, et au Chlamydosadre, je dois rappeler les belles figures qui ont été données du premier dans le $V_{o y}$. clans l'Inde, de Vict. Jacquemont (Rept., pl. x, mâle et fem.), et du second dans le Voy, au pôle sud et en Océanie, par Hombr. et Jacquinot (Rept., pl. vi). - Pour le genre Dragor (40), il faut mentionner les remarques de M. Schlegel sur l'espèce dite Dragon vert, par Daudin, et qui, selon le savant erpétologiste de Leyde, comprend 4 variétés de pays bien distinctes, qu'il désigne, d'après leur origine, comme Var. cle Java, de Sumatra, de Samarang et de Timor. Cette dernière, au reste, représente une espèce particulière : D. de Timor, Péron. - Nos collections n'ont reçu aucun échantillon nouveau du genre Lérolt́plde (4I), depuis l'époque où Cuvier l'a établi pour l'espèce unique dont les types furent des individus adultes et de jeune âge recueillis par Diard, en Cochinchine.

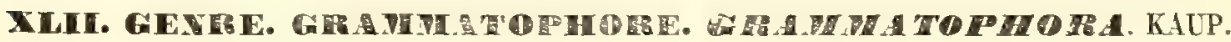

Le genre dont j'ai maintenant à parler offre cette particularité intéressante qu'il ne comprend, jusqu'à ce jour, que des Agamiens originaires de l'Océanie. Aux espèces plus ou moins anciennement connues, M. Gray en a joint une autre que le Muséum possède, et dont je dois présenter ici une courte description.

XLVIII. - 2 bis. Grammatophore orné, Grammatophota ornata, Gray. (Cat. of Liz., p. 253).

Idem, Cat. Rept. Mus. de Paris, p. 99. - Id., Id. Répert. Erpét. génér., Dum. Bib., t. IX, p. 279.

Écaillure dorsale homogène; sur la ligne médiane, après la petite crête très-peu saillante de la nuque, une rangée d'écailles plus grandes que les autres; de chaque coté du cou, 3 ou 4 petits groupes d'écailles tuberculeuses; squames des régions sus-orbitaires et postérieures de la tête plus petites que les antérieures.

Des deux espèces à écaillure du dos non entremêlée de tubercules 
(Gr. de Gaimard et Gr. de Decrès), c'est avec la seconde que celle-ci a le plus d'analogie par l'ensemble de sa conformation et par la disposition des écailles, qui sont petites et carénées sur le dos, mais lisses sur le ventre, ainsi que sur les flancs où elles sont également entremêlées de fort petits tubercules.

Les différences se rapportent surtout à l'écaillure de la tête, qui, au lieu d'être composée de pièces toutes de diamètre à peu près semblable, est formée par des squames très-fines, très-serrées sur les régions sus-orbitaires et occipitale, et beaucoup plus petites que celles de la portion antérieure de la tête. Toutes ces squames portent une petite carène, et particulièrement celles du museau qui sont comme tuberculeuses. En outre, le bord antérieur de l'oreille est armé de deux épines saillantes; puis, le pli cutané, de chaque côté du cou, a une forme angulense et non semi-circulaire. La disposition du système dentaire est la même que dans les deux autres Grammatophores à écaillure homogène.

Le spécimen unique de notre Musée n'a pas un système de coloration tout à fait semblable à celui du type décrit par M. Gray, car l'absence de l'épiderme dans une grande partie du dos laisse du doute sur les teintes dont il pouvait être orné pendant la vie, et en particulier sur les taches jaunes dont M. Gray a tiré la désignation vulgaire de ce Saurien, auquel il a imposé, en l'appelant Gr. ornata, une dénomination destinée à rappeler son aspect élégant. Sans aucun doute cependant, l'espèce signalée ici est identique à celle que le zoologiste anglais a, le premier, fait connaître. - La teinte générale, autant qu'on peut en juger par la tête, la queue et les membres, seules régions encore couvertes d'épiderme, est un brun jaunâtre, relevé sur la tête par des lignes noires, courbes sur le museau, obliques d'avant en arrière et parallèles entre elles sur les régions sus-oculaires. Sur la ligne dorsale, on voit une série de six à sept taches noires, dont le centre complétement décoloré maintenant, devait laisser paraître la couleur du fond ou peut-être une nuance plus claire. Sur la face externe des membres, il y a des bandes transversales noires; enfin, une série très-régulière de demianneaux également noirs, alternes, occupe la première moitié de la queue, dont l'autre portion porte des anneaux parallèles complets. Les parties inférieures sont d'un brun jaunâtre plus clair que les supérieures. Une grande tache noire couvre la poitrine 
Le reptile que je viens de décrire a été donné par M. le Prof. Nat. Guillot, qui l'avait reçu de l'Australie. - Sa longueur totale est de $0^{\mathrm{m}} 24$ (tête et tronc, oo8; quene, o ${ }^{\text {mi }}$ i6).

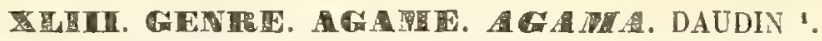

Aux dix espèces décrites par les auteurs en I 837 , on peut en joindre maintenant cinq nouvelles : $1^{\circ}$ Ag. nupta, Filippi, Giornale delle Instit. Lombardo, Milan, 1843, t. VI; $2^{\circ}$ Ag. colaticeps, Smith (Illustr., zool. S. Afr., pl. Lxxiv), et $3^{\circ}$ Ag. atricollis, Id. (1d., Appendix, p. I4); elles nous sont nconnues, mais les deux suivantes sont conservées dans nos collections.

\section{XliX. - Agame de Brbron, Agama Bibronii, A. Dum.}

(Cat. Rept. Mus. de Paris, p. 101.)

Agama.... Bib. MSS.

Quatrième doigt des membres postérieurs presque égal en longutur au troisième, ou mëme un peu plus court; dos à écaillure homogène; une petite créte sur le cou seulement; écailles du dos et des flancs carénées, celles des régions inférieures lisses et très-légèrement échancrées à leur bord postérieur; sur le chanfrein, une ligne longitudinale de quatre ou cinq écailles convexes, mais non carénées, plus grandes que toutes les autres plaques de la tete.

Des différentes espèces comprises dans ce genre, c'est l'Ag. des colons, quii offre le plus de ressemblance avec celle-ci, par la conformation de la tête, par l'aspect général de l'écaillure et par la présence, sur la nuque, d'une petite crête. I] faut néanmoins noter comme caractères importants et propres à l' $A g$. de Bibron: $1^{\circ}$ la forme arrondie, conique et non comprimée de la queue; $2^{\circ}$ la petite échancrure du bord postérieur des écailles gulaires

1. Je n'ai pas à rappeler ici la confusion qui a longtemps régné parmi les zoologistes relativement au sens qu'il convenait d'attacher au mot Agame comme clénomination générique, et relativement aux espèces qu'il devait servir à désigner. Je renvoie, pour ce sujet, aux remarques présentées par mon père et par Bibron, dans les généralités qui précèdent la description des espèces rapportées par eus à ce groupe (Erpét. gén., t. IV, p. 481-484). Il est arrivé, au reste, pour co nom trop vague, ce qui a eu lieu pour plusieurs autres, pour celui de Couleuvre, en particulier. Leur emploi a été restreint peu à peu, par suite des progrès ultérieurs de la zoologie. Ainsi, parmi les Agames, par excmple, un plus grand nombre d'espèces ayant été distingué, on a nécessairement trouvé entre elles des différences assez impurtantes pour qu'elles pussent être considérées, à bon droit, comme de véritables dissemblances génériques. On a donc, de cette façon, séparé les Grammatophores, les Stellions et les Phrynocéphales; et par voie d'exclusion, toutes les espèces qui ne pouvaient rentrer dans aucun c'e ces genres, ont conservé le nom primitif, dont la signification zoologique s'est trouvée alors bien plus

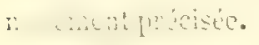




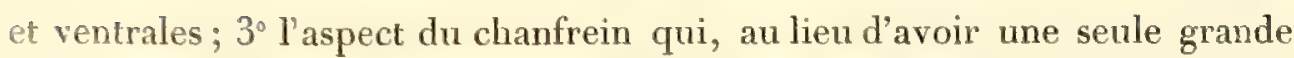
plaque en avant, comme chez l' $\mathrm{Ag}$. des colons, en porte, au contraire, cinq, bombées, mais non carénées, disposées en série linéaire continue depuis le bout du museau, jusqu'au. commencement de l'espace inter-orbitaire, et dont la troisième est la plus grande.

La plaque occipitale est médiocre et non entourée d'écailles pointues ou tuberculeuses. - L'oreille est grande et bien découverte; son bord antérieur est épineux; le supérieur et l'inférieur portent chacun un bouquet d'épines, et un peu en arrière du bord postérieur, il y en a deux. On en voit un de chaque côté de la nuque, au-dessus de l'oreille, et puis au delà, deux paires, l'une à droite et l'autre à gauche. - La peau forme, sous le con, deux plis transversaux, réunis sur la ligne médiane par un petit pli longitudinal. Au niveau de ces saillies cutanées et des enfoncements qui les séparent, les squames sont plus petites que partout ailleurs. Les ćcailles des membres sont carénées, et les sous-digitales sont munies chacune de deux carènes.

Toutes les parties supérieures sont d'un brun verdâtre, avec cinq ou six bandes d'un brun foncé, transversales, anguleuses et peu distinctement marquées, surtout les antérieures. Tel est leur aspect sur notre spécimen, mais chez l'un des deux individus observés à Londres par Bibron, au Musée de la Société zoologique de Londres, les bandes ont une couleur de brique mélée de brun. Des demi-anneaux bruns, pau réguliers, règnent sur toute la longueur de la queue, et les membres sont traversés, de distance en distance, par des bandes dont la teinte est semblable. Les régions inférieures sont d'un brun jaunâtre clair, sans aucune tache. Sur l'un des échantillons de Londres, la gorge est parcourue par sept ou huit bandes. longitudinales brunes.

Les types de cette espèce ont été rapportés du Maroc par M. le docteur Hay; l'on peut supposer que l'exemplaire donné au Musée de Paris par la Soc. zool. de Londres, provient du même pays.

Sa longueur totale est de $\mathrm{O}^{\mathrm{m}} 22$ ainsi répartis : tête et tronc, $\mathrm{o}^{\mathrm{nz}}$ og; queue, $a^{\mathrm{m}} \mathrm{I} 3$. 


\section{L. -5 bis. Agame sanguinolent, Agama sanguinolenta, Dum.}

(Cat. Rept. Mus. de Paris, p. 102.)

Lacerta agama Güldenstaedt (J.-A.) Note M. SS. que Pallas cite en entier. - Lac. sanguinolenta Pall. Zoogr. rosso-asiat., t. III, p. 23, pl. Iv, fig. 2. - Ag. aralensis Licht. Werzeich der doubl. des zool. Mus. zu Berlin, p. 101, no 29. - Ag. oxiana, Ejchwald Zool. spec. Ross., et Pol., 1839-30, t. III, p. 185. - Trapelus aralensis Eversm. Add. ad celeberr. Pall. zoogr. Ross.-asiat. 1835. - Trap. sanguin. Eichw. Fauna caspio-caucas. 1841, p. 89, pl. xiv, fig. 3 et 4.-Id., Berthold, Mor. Wagner Reise nach Kolchis und nach den deutsch. Colon. jenseist des Kaukasus. 1850, p. 330.

Quatrième doigt des membres postérieurs plus long que le troisième; queue conique; point de crête; écailles dorsales égales entre elles, et non semées d'épines ou de tubercules, toutes carénées, ainsi que celles des flancs et du ventre.

Quand on compare cette espèce nouvelle dans le Musée de Paris, avec celles qu'il possédait déjà, on voit que c'est à I'Ag. agile d'Olivier qu'elle ressemble le plus; mais celui-ci ne porte pas, comme l' $\mathrm{Ag}$. sanguinolent, des carènes sur les écailles des flancs et du ventre; en outre, les carènes des régions supérieures sont, chez ce dernier, bien plus prononcées et plus pointues; il résulte même de cette particularité que l'animal, surtout dans la partie antérieure du tronc, semble, en quelque sorte, hérissé. - Les plaques de la partie antérieure de la tête, au delà des narines et au devant des yeux, sont bombées et assez grandes; l'occipitale est fort petite et entourée d'écailles un peu rugueuses. Le tympan est moins grand, et par suite, moins visible que chez d'autres $A g$.; son bord supérieur est épineux; en arrière et à une très-petite distance, on voit un bouquet d'épines.

Il n'y a, sons la gorge, qu'un pli cutané ; il est très-apparent, et forme une courbe fort ouverte, à convexité postérieure, et dont les extrémités se portent vers l'angle de la mâchoire, d'où part le pli qui, comme dans presque toutes les autres espèces, se dirige en arrière au-dessus des épanles: chez celle-ci, il est très-marqué. La quene est conique, longue et effilée. On compte, à la mâchoire supérieure, 2 canines, 4 incisives et 32 molaires.

Les couleurs varient suivant l'âge. L'échantillon unique de la collection est adulte : il a les parties supérieures d'un brun olivâtre, plus clair en dessous. Sur les membres, on voit, mais peu distinctement, des bandes transversales foncées; celles de la queue sont plus apparentes; elles y forment des demi-anneaux. Notre spécienen est en bon état de conservation, et comme il 
ne reste plus aucune trace de couleurs vives, on peut supposer qu'elles caractérisent uniquement la livrée du jeune âge. Voici, d'ailleurs, comment elle est décrite par M. Eichwald, qui a fait figurer un jeune sujet : Régions supérieures d'un brun jaune et les inférieures jaunâtres. Sur le dos, trois séries régulières de taches : celles du milien bleues et bordées de brun, les latérales jaunâtres et également à bords bruns; flancs noirâtres et parsemés, çà et là, de taches jaunes inégales; tête d'un brun clair uniforme; lèvres blenes; gorge violacée, ou parcourue par des lignes longitudinales violettes.

Cet $A g$, dit le même zoologiste, habite la côte orient. de la mer Caspienne, dans le voisinage dı golfe Balkan ou dans les îles baignées par les eaux de ce golfe. On le trouve aussi près du lac Aral et du fleuve Oxus (d'où les noms d'Ag. Aralensis et Oxiana proposés par MM. Lichtenstein et Eichwald). 11 vit également sur les côtes orient. et septentr. de la mer Caspienne. C'est saus doute dans ces parages que notre individu, adressé de Saint-Pétersbourg par M. Ménestriés, aura été recueilli. - Sa longueur totale est de $o^{\mathrm{m}} 285$ (tête et tronc, $\mathrm{o}^{\mathrm{m}} \mathrm{I} 1 \mathrm{0}$; queue, $\mathrm{O}^{\mathrm{m}} 17^{5}$ ).

- Le Muséum n’a pas reçu de nouveaux Phrynocíphales (4/4), et il reste toujours quelques doutes sur plusieurs espèces décrites par M. Eichwald, dont la Faune contient de bonnes figures et des détails intéressants relatifs aux espèces déjà connues.

XLV. GENRE. STELLION. STELEUOD. DAUDIN.

A l'espèce anciennement connue et nommée St.vulgaire, M. Rüppell en a joint une autre qu'il a trouvée en Arabie ( St. cyanogastre). Une espèce trèsvoisine de cette dernière, si mème elle ne lui est identique, est signalée parmi les Reptiles de l'Inde par M. Blyth qui, sans se prononcer positivement à cet égard, propose le nom de St. indicus pour le cas où cette espèce serait vraiment nouvelle. Le St. du Caucase, qui est depuis peu reçu au Musée de Paris, et les St. clu Cap et caréné que j'ai fait connaître pour la première fois dans le Cat. des Rept., doivent être décrits. On peut, pour les distinguer les uns des autres, se servir avec avantage du petit tableau synoptique suivant :

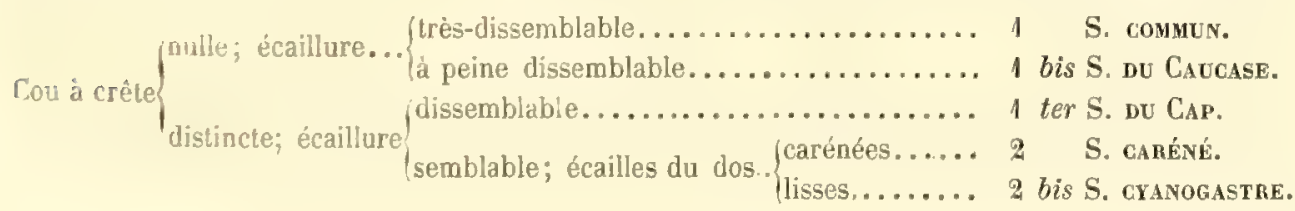




\section{LI. - I bis. Stellon du Caucase, St. caucasius, Eichw.}

(Zool. spec. Ross. et Polon. t. III, p. 187).

Lac, stellio Pall., et Lac. muricata Id. (non Lac. muric. Gm.) Zoogr. Rosso-asiat. t. III, p. 24 et p. 20, pl. rv, fig. 1. - St. vulgaris Ménestriés, Cat. raisonné, p. 64, n²19. - St. vulgaris Eversm. Lac. Imp. Ross. in Mém. Soc, impér. Moscou, t. III. - St. caucasius Eichw. Fauna Caspio-cauc., p. 80, pl. xiIn, fig. 1-8, pour les détails du squelette. - Idem, Gr. Cat. of Liz., p. 2ǒs. - Id., Dum. Cat. Rept. Mus. Par., p. 406. - Id., Id., Répert. Erpét. génér., Dum. Bib., t. 1X, p. 281.

Point de crete sur le cou; lécaillure presque semblable ou homogène, c'est-à-dire entremélée d'un très-petit nombre de tubercules disséminés, cà et là, sans ordre, et mousses pour la plupart; écailles du millieu du dos à peine carénées; celles de la queue fortement épineuses et formant des verticilles disposés comme les degrés d'un escalier.

Par sa conformation générale et surtout par l'aspect des verticilles de la queue, ce St. ressemble plus au $S t$. vulgaire qu'à tout autre; il est cependant facile de les distinguer. Chez le St. du Caucase, en effet, il n'y a, ni sur le dos, ni sur les flancs, des rangées longitudinales régulières de bouquets ou groupes de tubercules épineux; et l'on ne voit, sur ces régions, que des tubercules épars, très-peu proéminents, dont quelques-uns seulement se terminent en pointe. En outre, la tête, en arrière et sur les côtés, est garnie d'épines moins nombreuses et moins pointues. Enfin, les grandes écailles, sur le milieu du dos, sont tantôt lisses, tantôt faiblement carénées, et ne présentent pas la même irrégularité que celles du $S t$. vulgaire, dont les carènes, d'ailleurs, sont très-prononcées, surtout à la région postérieure du tronc.

La teinte générale est un brun olivâtre relevé de taches jaunes sur les flancs, mais particulièrement sur la région médiane du dos, dont les parties latérales portent, ainsi que les côtés du corps, de nombreuses maculatures ou lignes noires bordant quelques-unes des taches jaunes qui viennent d'être indiquées. Les couleurs sont plus claires en dessous; à la gorge, il y a des bandes noires, larges, souvent très-peu apparentes et parfois confondues les unes avec les autres. - La queue est jaunâtre, tachetée de noir et quelquefois de rouge, çà et là, sur sa face inférieure.

Cette espèce, dit M. Eichwald, se rencontre dans la Géorgie, dans l'Albanie, jusqu'à la mer Caspienne, près de Bakou et dans tout le désert du Mogan, ainsi que sur les montagnes de Talyschen où elle est abondamment 
répandue. - L'origine de l'un de nos exemplaires ne nous est pas connue, et nous manquons de renseignements précis sur les lieux où ont été recueillis les individus appartenant à cette espèce, et qui proviennent du voyage dans le Levant, et en particulier en Perse, entrepris par Aucher Éloy.

LII. - I ter. Stellion du Cap, Stellio capensis, A. Dum.

(Cat. Rept. Mus. de Paris, p. 106, et Répert. Erpét. génér., Dum. Bib, t. IX, p. 281.)

Cou surmonté d'une petite crête; écaillure du dos el des flancs hétérogène, c'est-à-dire entremélée de grandes écailles carénées, semblables à celles de la ligne médiane, et disposées en séries transversales, plus ou moins régulières; ćcailles caudales, de moyenne grandeur, formant des verticilles simplement imbriqués.

La tète est très-renflée en arrière des mâchoires, et il résulte de cet élargis sement que le cou, dont les téguments sont profondément plissés en dessous, semble fort rétréci. Elle ne porte pas de petits bouquets d'épines à ses régions postérieure et latérales, où l'on ne voit que de grandes écailles isolées à carène saillante et pointue. Outre les larges squames carénées de la ligne médiane du dos, à pourtour plus fortement dentelé que chez le St. vulgaire, il y en a d'autres sur les flancs, qui leur sont analogues, mais offrent cependant cette différence importante que l'arête en est moins proéminente, et qu'elles sont presque complétement lisses sur les bords. Elles forment des bandes transversales irrégulières, prolongées jusqu'au bas des flancs, et sur aucun point, on ne retrouve ces petits bouquets d'épines composés d'écailles pointues entourant un tubercule plus volumineux.

La petite crête assez apparente, si on la compare à celle des deux autres Sı. qui en ont également une sur le cou, est formée par une série longitudinale d'écailles étroites, mais saillantes et régulièrement disposées; elle s'étend depuis l'occiput jusqu'au delà des épaules._Les écailles de la queue faiblement carénées, et à pourtour dentelé en scie, ne forment pas des verticilles épineux et réguliers, comme chez les deux premières espèces qui, par ce caractère seul, pourraient être facilement distinguées des trois autres. Il faut encore signaler le peu de saillie dẻ la carène des grandes écailles sur les membres, ce qui est une particularité proprèà ce St. du Cap.

Le système de coloration consiste en une teinte brun-verdâtre en dessus, plus claire sur la ligne médiane, avec des nuances d'un vert clair assez marquées chez l'un des deux individus de la collection, et principalement à 
la région sus-céphalique. Ce dernier a la gorge également verte et le dessous du cou noir ; ces particularités se voient à peine sur l'autre individu, quoique tous les deux soient des mâles, car ils portent à la région préanale des écailles crypteuses, qui manquent dans l'autre sexe. Ils ont été rapportés du Cap de Bonne-Espérance par le voyageur Delgorgue. Le plus grand est long de $o^{\mathrm{m}} 3 \mathrm{I}_{7}$ (tête et tronc, $\mathrm{o}^{\mathrm{m}} \mathrm{I} 37$; queue, $\mathrm{o}^{\mathrm{m}} \mathrm{I} 80$ ). L'autre est à peine plus petit.

LiII. - 2 bis. Stellion Caréné, Stellio carinatus, A. Dum.

(Cat. Rept. Mus. de Par., p. 107, et Répert. Erpét. génér., Dum. Bib., t. IX, p. 281.)

Cou surmonté d'une petite crête; écaillure du dos et des flancs homogène, c'est-à-dire non entremélée de tubercules ou d'épines; grandes écailles du milieu du dos disposées de façon que leurs carènes forment des rangées obliques convergeant vers la ligne médiane; écailles de la queue à épines peu saillantes et irrégulièrement verticillées.

L'arrangement très-régulier des stries obliques formées par les carènes des grandes écailles médianes du dos éloigne ce $S t$. de tous ses congénères. Leur bord postérieur est à peine dentelé. - Les écailles caudales ont une carène terminée par une épine peu saillante et leur bord postérieur, de même que chez les St. clu Cap et cyanogastre, n'étant pas rectiligne, il en résulte, leurs dimensions, d'ailleurs, restant moindres que chez les St. vulgaire et du Caucase, qu'elles ne sont pas disposées en grands verticilles parfaitement réguliers comme chez ces deux derniers. Cette différence se remarque principalement dans la première moitié de la queue.

Les membres sont revêlus d'écailles carénées, toutes semblables entre elles et sans épines saillantes.

Autour de l'oreille, on voit cinq groupes isolés d'épines longues et pointues; il y a deux groupes semblables, placés, l'un au devant de l'autre, de chaque côté du cou, et un troisième plus bas et plus en dehors, précisément au-dessous des lèvres. - Deux plis transversaux, réunis au milieu par un troisième pli vertical, occupent le dessous du cou, dont l'écaillure est granuleuse.

Autant qu'on peut en juger, d'après l'un des trois exemplaires du Muséum, sur lequel l'épiderme est le moins détruit, la couleur générale est une teinte olive à reflets jaunâtres, ornée de petites taches noires sur le dessus et les còtés du tronc. La queue, d’un brun jaune assez clair à sa base, devient noire 
dans une partie de son étendue, puis reprend, au delit, son premier aspect.

Un individu, plus grand que les deux autres, et qui offre, malgré quelques légères différences peu importantes, il est vrai, tous les caractères de l'espèce nouvelle, dont il ne semble pas pouvoir être éloigné, a une teinte noirâtre générale. Ils ont tous été rapportés par Aucher-Éloy, au retour de son voyage en Perse et dans le Levant.

La longueur totale du plus complet des deux exemplaires, dont l'identité, du reste, est parfaite, est de $0^{\text {ni }} 395$ (tête et tronc, $o^{\text {nx }} 130$; queue, $o^{\text {nn }} 265$ ).

- Je n'aurais pas à parler du genre Fodette-queue (46), dont l'espèce la plus commune, souvent observée vivante à la Ménagerie, habite les provinces méridionales de l'Algérie et l'Égypte ( $\boldsymbol{F}$. ou Uromastix acanthinure), si, dans ces derniers temps, une addition n'avait été faite à ce groupe. Elle est due à M. le Prof. Valenciennes, qui a décrit sous le nom de Fouettequeue iemporal (Uromastix temporalis), un individu adressé au Muséum par M. le marćchal Vaillant. Il en a donné la diagnose suivante (C. rendus des séances de l'Acactémie des sciences, I854, t. XXXIX, p. 89): Ur. temporalis corpore ex viridescente griseo; dorso maculis parvis, subrufis, consperso; squamis quadratis ad tempora quatuor.

Ce Saurien, désigné par les Arabes sous le nom de Dobb, a été trouvé dans le grand désert de Sahara, entre Aquebly et Djebbel-Hoggar.

XEVH. GENRE. MOLACH. MEROCH. GRAY.

(Grey's travels, W. Aust.)

Tronc, queue et membres revêtus d'écailles granuleuses, entremêlées de longues épines, dont les plus considérables se voient sur les régions sus-oculaires, où elles simulent une paire de cornes; sur le cou, une volumineuse protubérance presque sphérique couverle d'épines.

L'aspect général de ce Saurien est extrêmement bizarre, car il est partout hérissé d'épines longızes et pointues, bien plus nombreuses et plus fortes que celles des Phrynosomes, qui sont, parmi les Iguaniens acrodontes, les analogues du Moloch. Ce dernier diffère plus encore des genres à la suite desquels il est placé, que les Phrynosomes, malgré les singularités de leur écaillure, ne s'éloignent des autres Pleurodontes.

Une seule espèce, jusqu'icí, est décrite par les naturalistes, et les particulaArchives du Muséum. T. Vill. 
rités qui la distinguent, doivent être exposées avec quelques détails, car elles font connaitre l'étrange physionomie de ce Reptile de l'Océanie, d'où l'on avait déjà reçu diverses espèces de Sauriens remarquables par des anomalies de structure ${ }^{1}$.

LIV. - 1. Moloce hérissé, Moloch horridus, Gray (loc. cit.). Idem, Gr., Cat. of Liz., p. 263. - Idem, A. Dum., Cat. Rept. Mus. de Paris, p. 109.

D'un brun tirant sur le ronge, un peu moins foncé en dessous qu'en dessus, où il est orné de grandes taches noires; sur les régions inférieures, des taches rougeâtres à bordure sombre.

L'animal est trapu et ramassé, la tête courte, le museau très-obtus; la queue et les membres sont peu développés. Dans tout l'ensemble de la conformation générale, il y a une certaine analogie avec les Phrynosomes. Le cou, bien distinct du tronc, porte, en dessous, un pli garni à son bord libre, de tubercules faiblement épineux, et en dessus, une volumineuse protubérance surmontée d'écailles pointues.

Le revètement squameux du tronc se compose de pièces fort inégales entre elles, toutes légèrement bombées et granuleuses, car elles sont surmontées de petites aspérités. Sur un très-grand nombre, il y a, dans le point le plus saillant, une pointe épineuse plus ou moins aiguë, mais souvent très-développée au sommet des écailles plus hautes que les autres, qui entourent la base des grandes épines. - Parmi ces épines fortes et à pointe acérée, qui forment une puissante armure défensive au Moloch, les plus longues sont celles des crêtes surciliaires. On en voit une, en effet, au-dessus de chaque oil, longue de $0^{\text {ma }}$ o I 2 à $\mathrm{O}^{\mathrm{m}}$ o 15 dirigée en haut et dehors; sa base est volumineuse et placée au milieu d'écailles pointues plus petites, disposées comme les sépales d'un calice. Sur la nuque, il y a sept épines formant un collier immédiatement suivi de la protubérance irrégulièrement sphérique de la région cervicale, et dont le diamètre est de $o^{\text {na }}$ or environ. Sa cir-

4. Tels sont les Scincoîdiens nommés Trachysaures, et qui ont l'écaillure des régions supérieures composée de pièces osseuses fort épaisses, rugueuses, formant, par leur ensemble, une sorte de carapace. Tel est aussi le Silubosaure, dont la queue courte et déprimée est revêtue de longues et fortes épines, comme l'enveloppe du fruit du châtaignier. L'espèce qu'il faut surtout citer est le grand Iguanien acrodonte à large collerette plissée connu sous le nom de chlamydosaure. On sait, d'ailleurs, combien les Ornithorbynques, les Kangurous et autres marsupiaux, ainsi que l'Aptéryx, originaires de.l'Australie, diffèrent de tous les mammifères et de tous les oiseaux connus. 
conférence est hérissée de petites épines; deux de celles-ci cependant, sont beaucoup plus grandes, et marquent le commencement de deux séries parallèles d'épines étendues, de chaque côté de la ligne médiane, depuis ce point jusqu'à l'extrémité de la queue. En dehors des séries que je viens d'indiquer, et à droite, comme à gauche, il y en a quatre autres également parallèles, de dimensions graduées de telle sorte que les deux rangées les plus externes, qui sont en même temps inférieures, parce qu'elles protégent les flancs, sont composées d'épines plus longues que celles des deux séries latérales du dos; ces dernières ont elles-mêmes plus de hauteur que les deux rangs internes, dont la séparation est indiquée par la ligne médiane. De la régularité parfaite dans la disposition mutuelle des écailles épineuses, il résulte que le tronc est revêtu, en dessus, de bandes longitudinales et transversales d'épines. Celles des flancs ne se voient que sur le tronc, mais les dorsales, deux de chaque côté, se continuent sur les faces supérieure et latérales de la queue. Les membres portent trois ou quatre rangs d'épines semblables aux précédentes. - Toutes les régions inférieures sont moins bien armées. On n'y remarque plus, au milieu des écailles granuleuses, que des écailles à carène saillante et plus ou moins aiguës à leur extrémité postérieure, qui se relère en pointe, mais elles ne sont jamais aussi acérées, ni aussi longues que les aiguillons du dos et des côtés du trone. Les doigts sont courts et couverts en dessus, comme en dessous, d'écailles à forte carène.

On compte, à la màchoire supérieure, 4 incisives, 2 canines et 26 molaires inclinées en dedans.

De chaque côté du cou, une tache étroite, commençant à la nuque, couvre la moitié correspondante de la protubérance cervicale, et se continue un peu au delà de l'épaule. Sur le dos, à une égale distance des membres antérieurs et des postérieurs, de l'un et de l'autre côté de la ligne médiane, on en voit une large et irrégulière, puis deux allongées et beaucoup plus petites, au niveau du bassin, et qui sont suivies de deux autres aussi étroites, mais plus longues. Quoique très-rapprochées vers le milieu du cou et du dos, toutes ces taches, d'un noir profond, sont séparées dans ce point par un très-petit espace où apparaît la teinte brune. Le milieu et les côtés de la queue sont, comme les flancs, irrégulièrement tachetés de noir. Les membres portent de larges bandes obliques et noirâtres. - Sur la poitrine, il y a une tache de teinte rougeâtre et foncée; elle est divisée en arrière et en- 
tourée par une ligne sombre. D'autres taches, de couleur semblable, mais d'une forme différente et irrégulière, couvrent en partie le ventre; l'une située en avant, beaucoup plus large que longue, l'autre en arrière, de forme allongée, s'étend sous la queue où elle en précède deux d'apparence semblable.

Le Muséum possède trois beaux échantillons de cette espèce rare et curieuse. Ils sont parfaitement semblables les uns aux autres. Il y en a deux, en particulier, conservés dans l'alcool, qui ont été recueillis par M. J. Verreaux, dans la province de la Rivière des Cygnes (Nouv.-Hollande).

Le plus grand est long de $o^{\mathrm{mm}} \mathrm{I} 65$ (tête et tronc, $\mathrm{o}^{\mathrm{nn}}$ o8g; quene, $\mathrm{o}^{\mathrm{m}}$ o 76 ).

- Ici, se termine la grande famille des Igunariens, dont j'ai eu à décrire trente-quatre espèces peu connues ou entièrement nouvelles cómprises dans les riches collections du Musée de Paris et dont plusieurs sont devenues les types de treize genres nouveaux, ainsi que d'un sous-genre spécial parmi les Galéotes. Les espèces qui y sont inconnues ont été seulement mentionnées.

En ajoutant à ces additions celles qui se rapportent aux familles des GECкотіеss et des Varaniens, il y a, dans ce travail, 54 espèces nouvelles, dont 25 que j’ai nommées, parce qu'elles n'avaient été indiquées par aucun zoologiste ${ }^{4}$. Parmi ces dernières, il s'en trouve quatre, qui n'ayant pu être rap. portées à aucun des genres déjà établis, ont pris rang sous des dénominations génériques nouvelles : (Ophryessoïde, A. Dum.; Sauromale, Id.; Phymatolépide, Id,, et Arpéphore, Id.).

Le relevé de mon précédent Mémoire (Arch. du Mus., t. VI, p. 209-264, pl. xıv-xxir) montre que pour les Chéloniens, les Crocodiliens et les CaméLÉoxs, 23 espèces nouvelles ou peu connues, dont sept jusqu'alors inédites, jointes aux 54 autres, forment pour la portion encore peu considérable de la classe des Reptiles maintenant passée en revue, dans ces premiers Suppléments de l'Erpét. génér. de mon père et de Bibron, un total de 77 espèces qui sont décrites, et pour la plupart figurées sur i 7 planches, dans ces Archives publiées par les soins de l'administration du Muséum d'histoire naturelle.

Un troisième Mémoire comprendra les additions relatives aux derniéres familles de l'ordre des Sauriens.

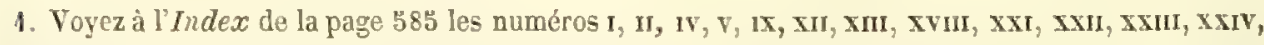

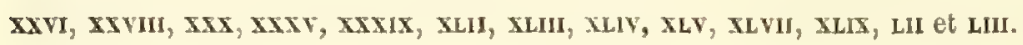




\title{
INDEX
}

\author{
DES
}

\section{GENRES ET ESPÈCES DE REPTILES}

DÉCRITS, FIGURÉS OU INDIQUÉS DANS CE MÉMOIRE'.

ORDRE DES SALRIENS.

III. Gechotiens of Ascalabotes.

I. Platydactyle à venPages. Plinclies.

tre rude, $\mathrm{A}$. Dum. 452 XVII, 1.

II. P. de Boivin, A. D. 4 šl XVIII, 2.

III. $P$. de l'Océan $P \alpha$ -

cifique, D. (Gray). 455

P. de Reeves, D. (Gr.) 457

IV. Hémidact. taches-

rousses, A. Dum.. 461 XVII, 2.

V. H. atèle, A. Dum.. 462 XVIII, 9.

VI. Phyllodact. d'Eu-

rope, Gené....... 465

V11. P.spinigère, D. (Gr.) 467 Sphériod. bisarre,

Cuv, et Variétés. 469 XVII, 3,4 .

VIII. Gymnodact, humé-

ral, Guich...... 474

IX. G. varié, A. Dum., $470 \%$

I. G. brun, A. Dum.

(Stén. Hallow.). . 477

XI.G. élégant, D. (Gr.). 477 XVIII, 14.

III. G.d'Arnoux, A. D. $479 \mathrm{XVII}, 5$.

XIII. G. de Perse,A. Dum. 481

XIT. G. caspien, Eichw. 482

$\mathrm{XV}$. G. coléonyx, A. D.

$$
\text { (Gray)........ } 483 \text { XVII, } 6 .
$$

XVI. Sténodact, mauri-

tanique, Guich... 487
XVII. S.babillard, Smith.. 488

Pages. planches.

XVIII. S. queue-cerclée, $\mathbf{A}$.

$$
\text { Dum......... } 489 \text { XVIII, } 15 .
$$

IV. Varaniens ou Platynotes.

Héloderme hérissé,

Wiegm ......... 491

XIX. Varan ponctué, D.

(Gray)........4 497

XX. $Y$. de Gould, Dum.

(Schl.)........498

$\boldsymbol{V}$. vert et $\boldsymbol{V}$. de $\mathbf{D u}$ -

méril, S. Nüller... 498

V. Iguaniens ou Eunotes.

A. Igdan, pleurodontes.

Laimancte longipède,

Wiegm......... 512 XXI, 4 .

XXI. Anolis à bandes

transv. A. Dum. 515 XIX, 3

XXII. An. hétéroderme,

A. Dum....... $\$ 16$ XIX, 4.

XXIII. Corytoph très-ca-

réné, A. Dum.... $518 \mathrm{XX}_{3} 3$.

XXIV. Basilic à bonnet,

A. Dum......... 522 XXI, I.

B. à bandes, Wiegm. $523 \mathrm{XXI}, 3$.

B. à capuchon, Daud. 524 XXI, 2 .

Amblyrhynque à

crête, Bell....... "̈24

A. de Demarle, D.B. 525

1. Les espèces précédées d'an naméro d'ordre sont décrites; les autres sont senlement indiquées avec plus ou moins de détails. L̇ assez grand nombre d'espèces inconnes an Musée de Paris, et dont la description a été donnée par différents zoologistes, esı signalé dans ce Mémoire, mais elles n'ont pas dú être portées à la table. 
Cyclure pectiné, Pages. Planches.

Wiegm........ 526

Enyale rhombifère,

Wagl......... 527

En. à deux raies,

Dum. Bib........ 528

XXV, E. tête-large, Guich, 529 GENRE OPHRTESSOÏDE. 530

XXVI. O.trois-crétes, A.D. в31 XXII, 1. gente Crotaphyte,

Holbr.......... 532

Léiosaure (Crotaph.)

à collier, A. Dum. 532 XXII, 3. (sous le nom de L. trapu).

L. de Bell, Dum. Bib. 533 XXII, 2. GENRE DiPLOLÈME,

Bell............ 534

XXIII. D. de Bibron, Bell.. 534

D. de Darwin, Bell. Б3อ

gente Sauromale,

A. Dum........ 53

XXiII. S. sombre, A. Dum. 536 XXIII, 3.

XXIX. Holotropide (Léio-

céph.) de Gray, D. 538

XXX. H. téte-rude, A. D. 539 XXIII, 1.

XXXI. Proctotrète mosaïque, Hombr. Jacq. 5 st

XXXII. Pr.grêle, Bell.....542

XXXIII. $P_{r}$. de Magellan,

Hombr. Jacq..... . b43

geNRe Holbrookid,

Girard........... 㢮

XXXIY. H. tachetée, Girard. 546

GENRE UTA, Baird

et Girard.......... . $5 \mathfrak{\xi} 8$

gevre Phthatolé-

PIDE, A. Dum..... 548

$\mathrm{XXXV}, \mathrm{Ph}$. deux-carènes,

A. Dum........ 549 XXIII, ə.

GENRE AKOTA, Hall. 5ö
XXXVI. Phrynosome à tete- Pages. Planches.

plane, Hallowell.. 552

XXXVII. P. de Douglas, Bell. 554

genre Homalosadre,

Hallowell ........ 555

GeNRE CentruRe, Bell. כ็้อ

XXXVIII. C. Alagellifère, Bell. 557 XXII, 5 .

XXXIX. C. quatre-taches,

A. Dum......... 558 XXII, 4.

XL. Doryphore tête.

jaune, Guich..... 560

geNRE Hoplocerque,

Fitz.......... 561

XLI. H. épineux, Fitz... 562

B. Iguan, acrodontes.

S.-GENRE MÉCOLÉPIDE

A.D. (Salea Gray), 564

XLII. M.tri-épineux,A.D. 564 XXIV, 1.

XLIII. M. hérissé, A. Dum. $566 \mathrm{XXIV,} \mathrm{2.}$

XLIV. M. sillonné, A. D.. $567 \mathrm{XXIV,} 3$.

$\mathrm{XLV}$. Lophyre spinipède,

A. Dum.........568

XLVI. L. épineux, Hombr.

Jacq..........5 569

GENRE ARPÉPHORE,

A. Dum....... 570

XLVII. A. à trois-bandes,

A. Dum.........s7t

XLVIII. Grammatophore

orné, Gray...... 5.

XLIX. Agame de Bibron, A. Dum.......... 57t

L. A. sanguinolent, A. Dum. (Pallas)..... 5:6

LI. Stelliondu Caucase, Eichrvald. ........ 578

LII. S. clu Cap, A. Dum. รั79

LIII. S. caréné, A. Dum. s\$o Fouette-quere tem. poral, Valenc... 581 GENRE Moloch, Gray. 581

LIV. Moloch hérissé, Gr. 582 


\section{EXPLICATION DES PLANCHES}

Flasche XVII, - 1. Portion de l'abdomen du Platydactyle ventre-rude, A. Dum., p. 452.

Pages $439-490$. Geckotiens.
- 2. Hémidactylo taches-rousses, A. Dum., p. 461. - 3. Sphériodactyle bizarre, Cuv.; $3 a$ et $3 b$, sa tête vue en dessous et une portion du dos; $3 c$, tète du mème (variété blonde) vue en dessus; 4 , le mème (variété à taches noires); $4 a$, sa tête vue en dessous, p. $468.470 .-5$. Gymnodactyle d'Arnoux, A. Dum., p. $479 ; 5 a$, l'un de ses doigts; $5 b$, portion du dos du même. - 6. Coléonyx élégant, A. Dum. (Gray), p. $483 ; 6 a$, sa main vue en dessous; $6 b$, l'un des doigts; $6 c$, région anale du mème.

Puivene XVIII. - 1, $1 a$, Platydactyle de Duvaucel, D. B. - 2, 2a, 2 b, Platydactyle de Pages $\$ 39-490$. Geckotiens.

(Pieds et doigts 2 et 3 fois la grandeur naturelle.) Boivin, A. Dum., p. 45 4. - 3. Platydactyle théconyx, D. B. $-4,4 \alpha$, Platydactyle des murailles, D. B. - 5. Platydactyle des Seychelles, D. B. - 6, $6 a, 6 b$, Hémydactyle mabouia, Cuv. - 7. Hémidactyle tacheté, D. B. - 8. Hémidactyle Oualien, D. B. - 9. Hémidactyle atèle, A. Dum., p. 462. - 10, $10 a$, Ptyodactyle frangé, Cuv. - 11, $11 a$, Phyllodactyle porphyré, D. B. - 12, $12 a, 42 b$, Sphériodactyle très-petits-points, D. B. - 13, $13 a$, Gymanodactyle gentil, D. B. 14. Gymodactyle élégant, A. Dum. (Gray), p. 47\% = 4 o. Sténodactyle queue-cerclée, A. Dum., p. 489. - 16, $16 a$, Sténodactyle tacheté, Cur.

Prarche XIX. - 1. Main d'Anolis resplendissant vue en dessous, Schl. - 2. Anolis de Iguan. pleurodontes (Anolis). Valenciennes, D. B. - 3. Anolis à bandes transversales, A. Dum. p. 51b. - 4. Anolis hétéroderme, A. Dum, p. $516 ; 4 a$ et $4 b$, tête et portion des téguments du mème.

Puaxche XX. - $1,1 a, 1 b$, Corytophane á crête, Boie; $1 c$, dents du même. - a. CoryIguan. pleurodontes

(Corytophanes

et Basilics).

PLATChe XXI. Iguan. pleurodontes (Basilics

et Laimanctes). tophane caméléopside, D. B. (d'après Gravenhorst: Chamxleopsis Hernandesii). - $3,3 a$, Corytophane très-caréné, A. Dum. p. อ̆18. — 4 \& $a$, Tête et dents du Basilic à bandes, Wiegm.

- 1 . Basilic à bonnet, A. Dum., p. 322; $4 a$, dents du même; $1 b$, tête de la femelle. - 2. Tète du Basilic à capuchon, Daud., femelle. — 3. Tête du Basilic à bandes, Wiegm. - 4 et $4 a$, tête du Laimancte longipède, Wiegm., vue en dessus et de profil. 
Plaxche XXII. Iguan. pleurodontes (ophryessoìde, Léiosaures, Centrures).

Playche XXIII. Jguan. pleurodontes (Holotropide, Phymatolépide, Sauromale).

Plaxche XXIV. Iguan. acrodontes (Mécolépide).
- 1. Ophryessoïde trois-crêles, A. Dum., p. 5331. - 2. Tête du Léiosaure de Bell, D. B. - 3. Tête du Léiosaure å collier (Crotaphyte à collier, Holbrook) nommé, à tort, sur la planche Léiosaure trapu.-4. Centrure quatre-taches, A. Dum., p. 5วั8; $4 a$, tête du même. - 5. Tête du Centrure flagellifère, Bell, p. 557.

- 1. Holotropide tête-rude, A. Dum., p. b39; $1 a$, la tête vue en dessus. - 2. Phymatolépide deux-carènes, A. Dum., p. 5449;2 $a$, la tête vue en dessus; $2 b$, portion des téguments amplifiée. - 3. Sauromale sombre, A. Dum., p. 535; $3 a$, la tête vue en dessus.

- 1. Mécolépide tri-épineux, A. Dum., p. $564 ; 1 a$, portion des téguments. - 2, M. hérissé, A. Dum., p. $566 .-3$, M. sillonné, A. Dum., p. 567. - 4, Portion des téguments du Bronchocèle à crinière, D. B. - 5, Id., du Galéote versicolore, D. B. (Les détails sont du double de la grandeur naturelle. 

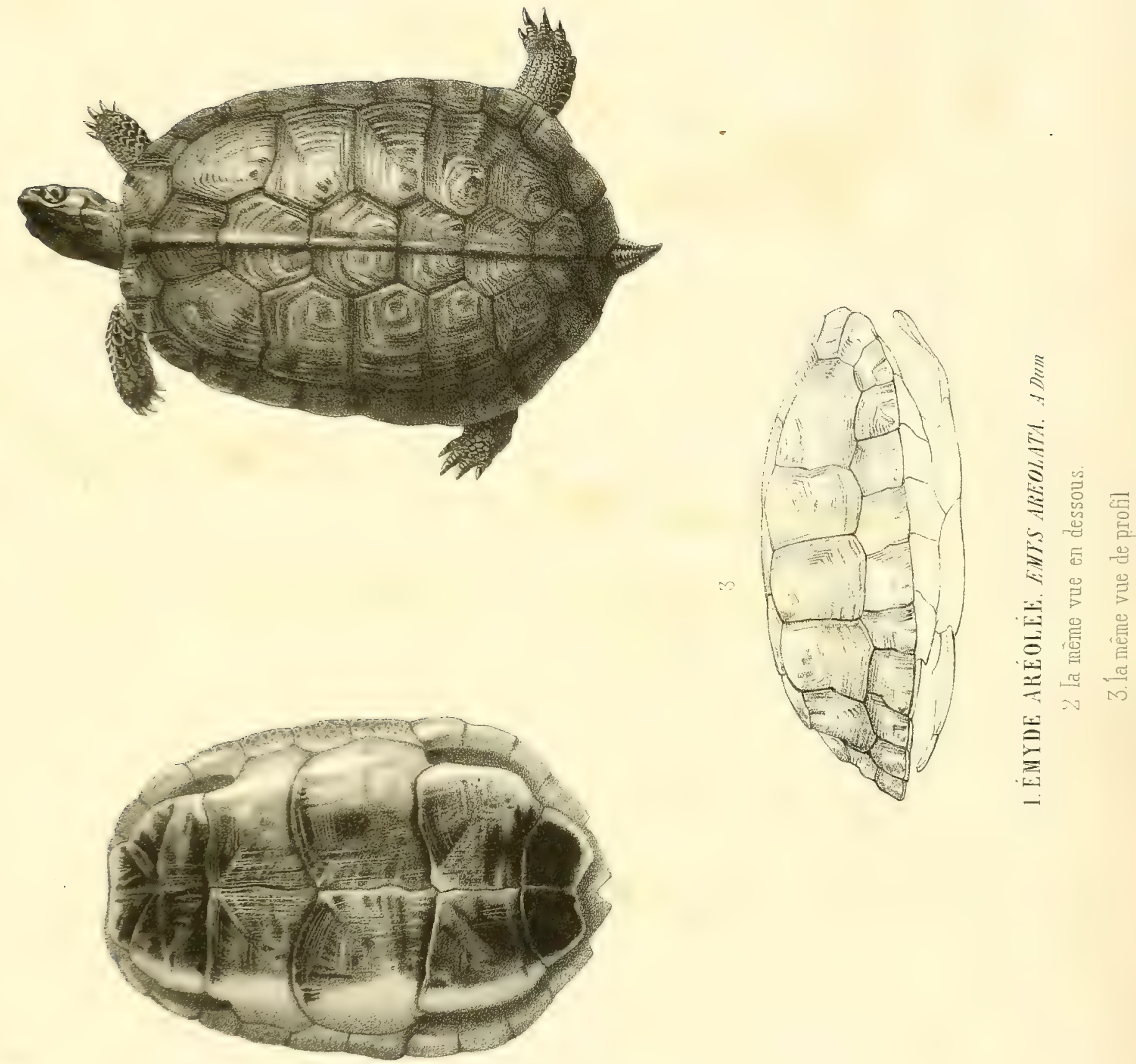



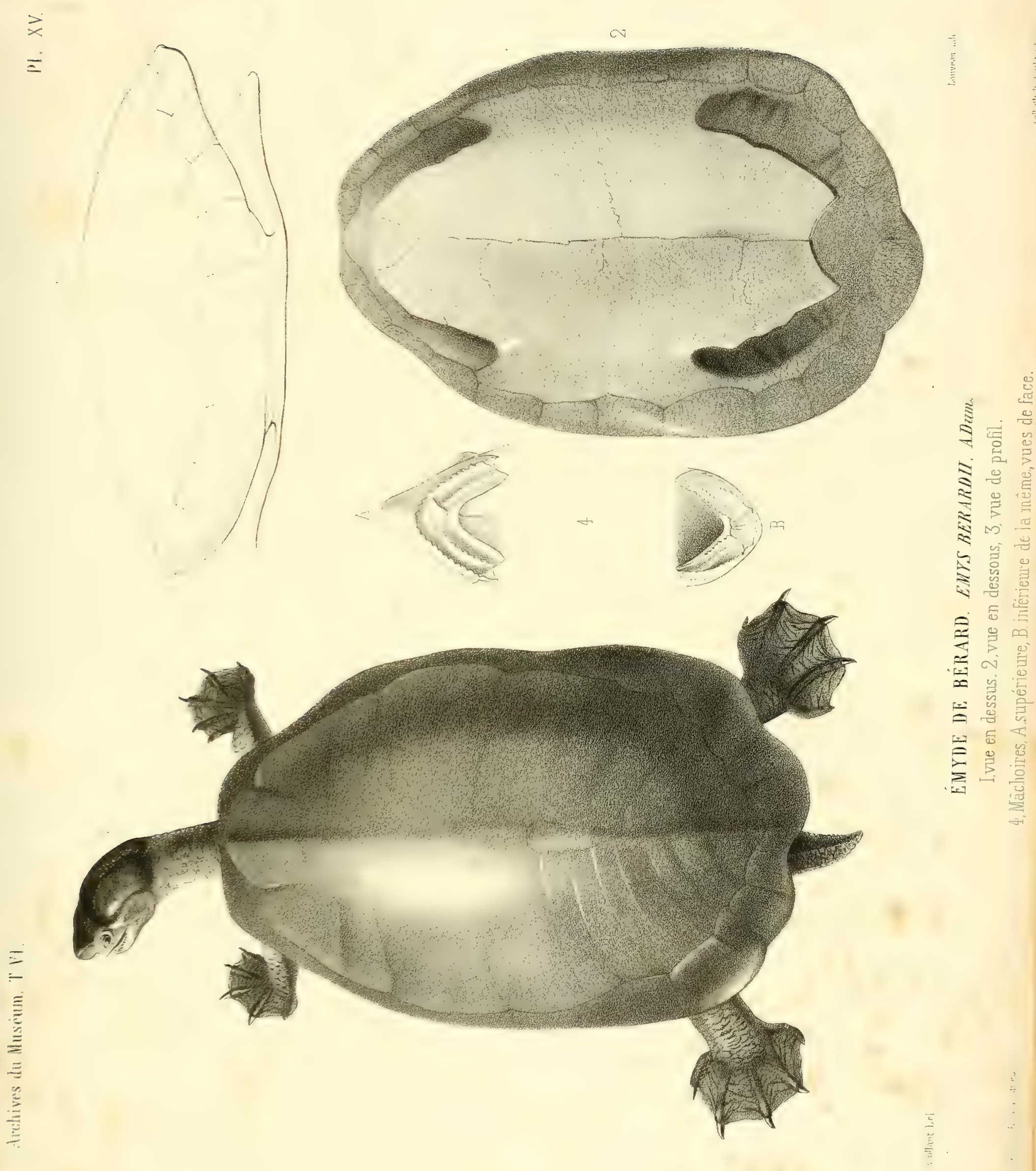




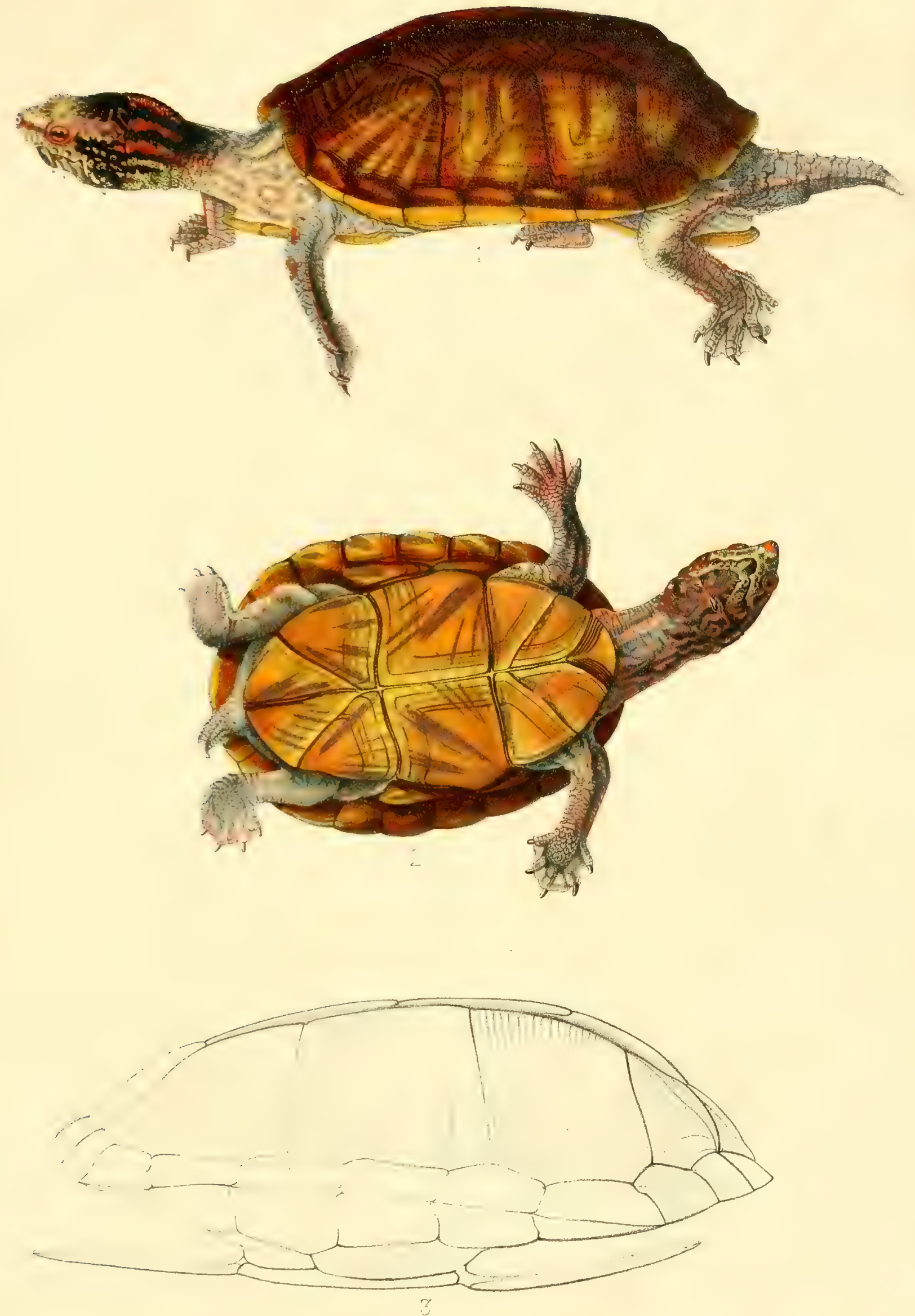

1. CINOSTERNE ENSANGLANTE. CINOSTERNON GRUENATUM. Dum Bit.

Vaillant Lith eIl dessus 



$$
-
$$





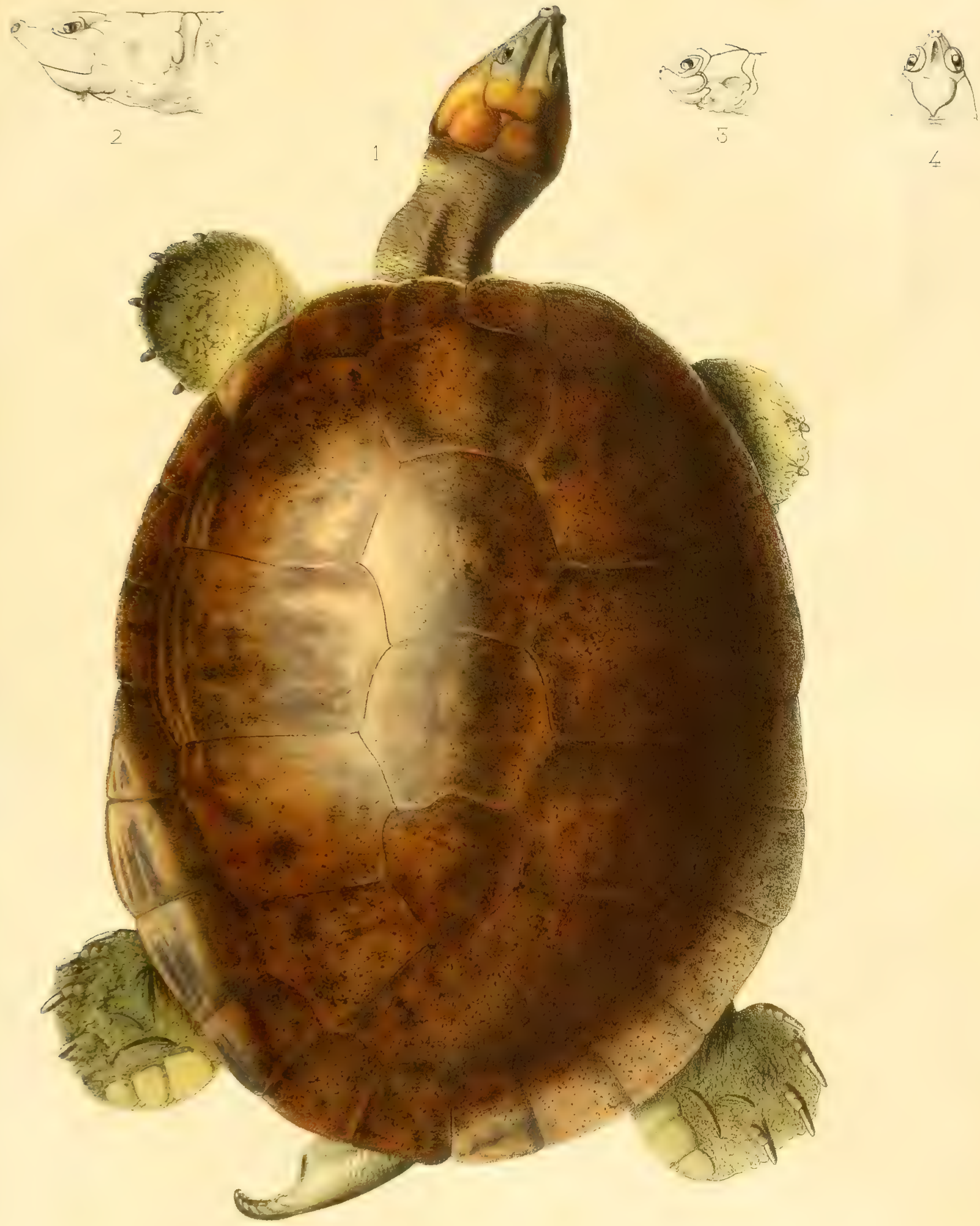

1. PODOCNEMIDE DE LEWY, PODOCNEMIS LEWYANA. A. DUUn

(Vue en dessus.) 


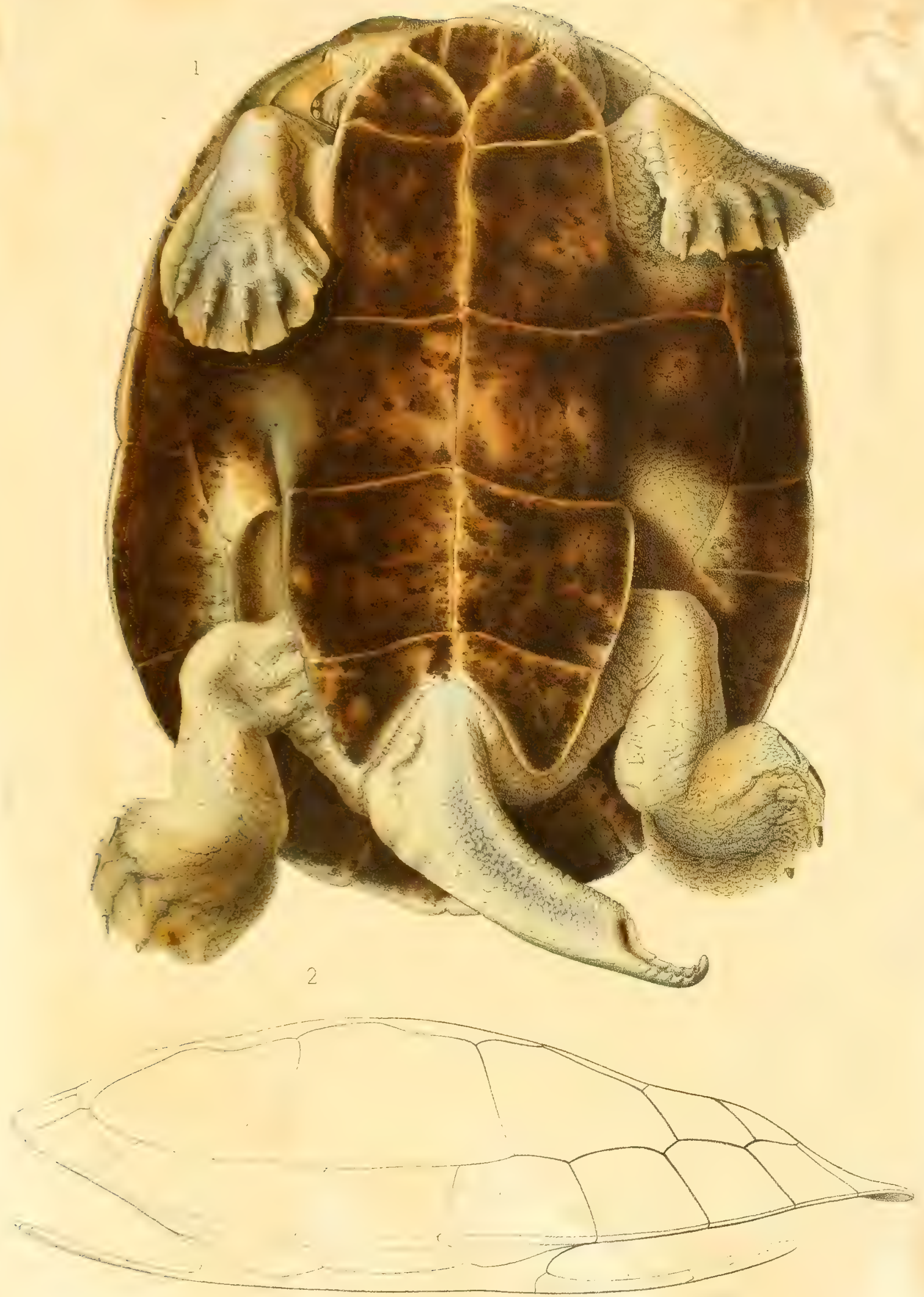

Vollont lith

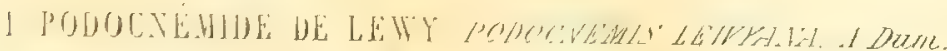

(Vue en dessous, 



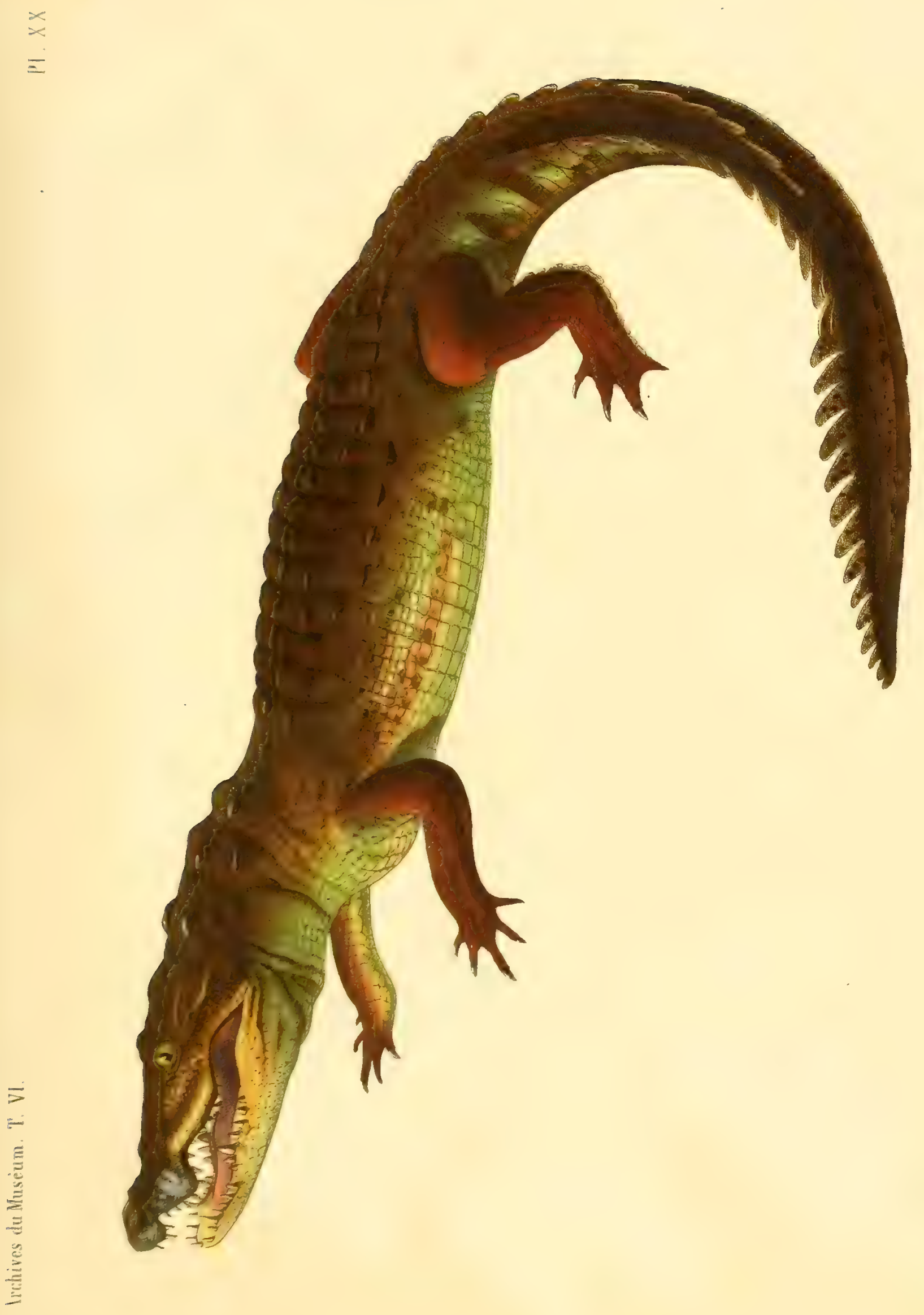

ह 



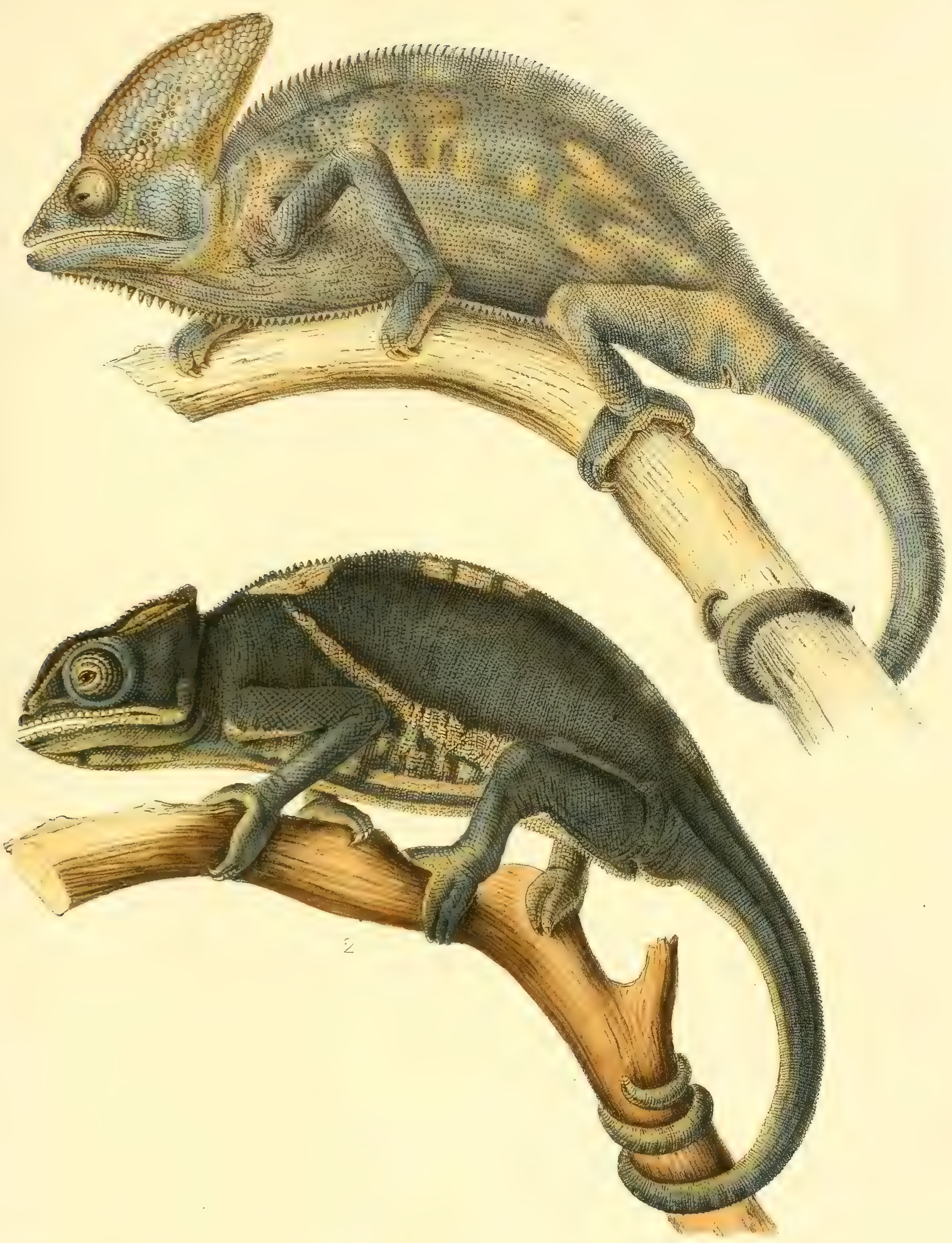




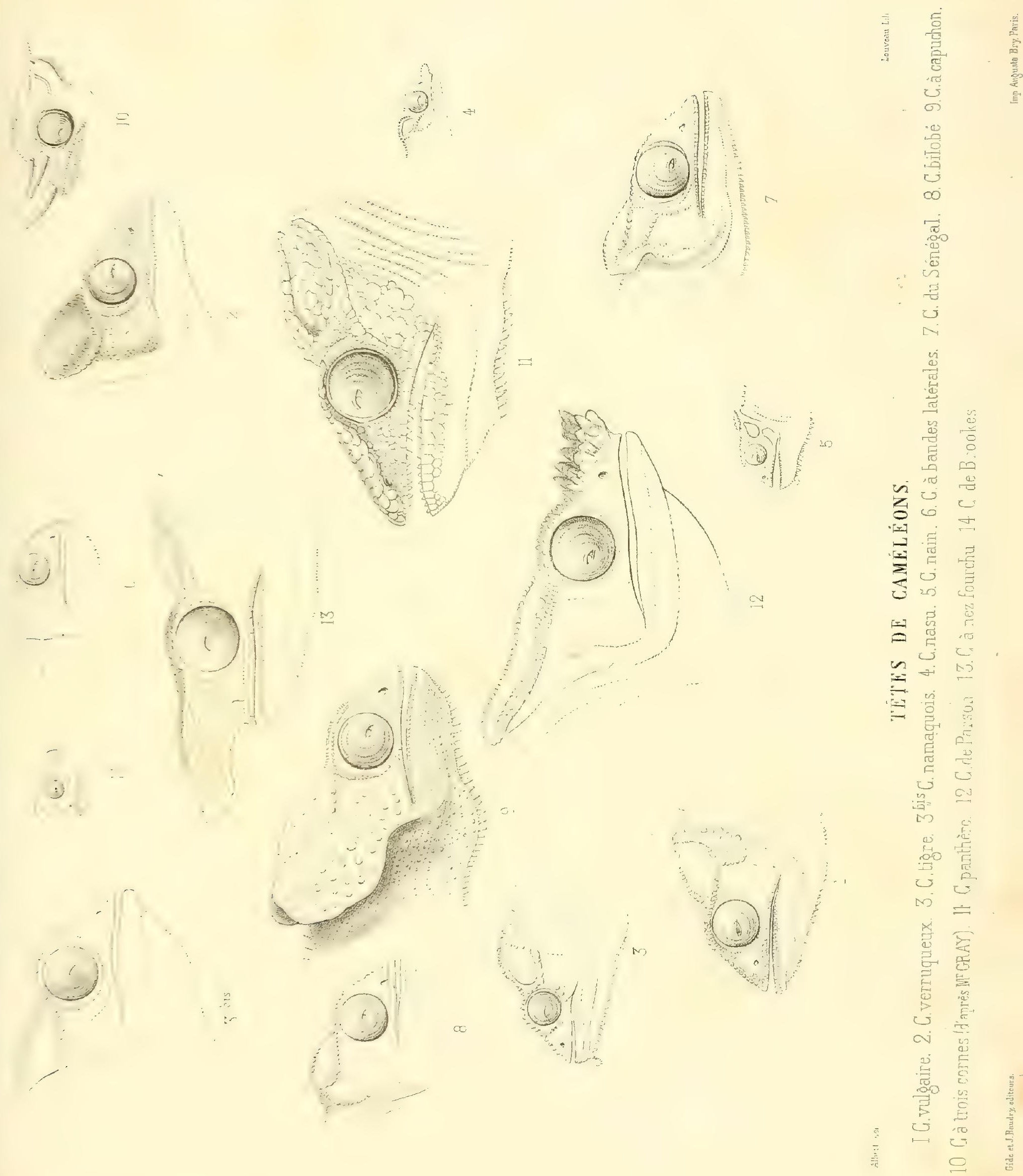


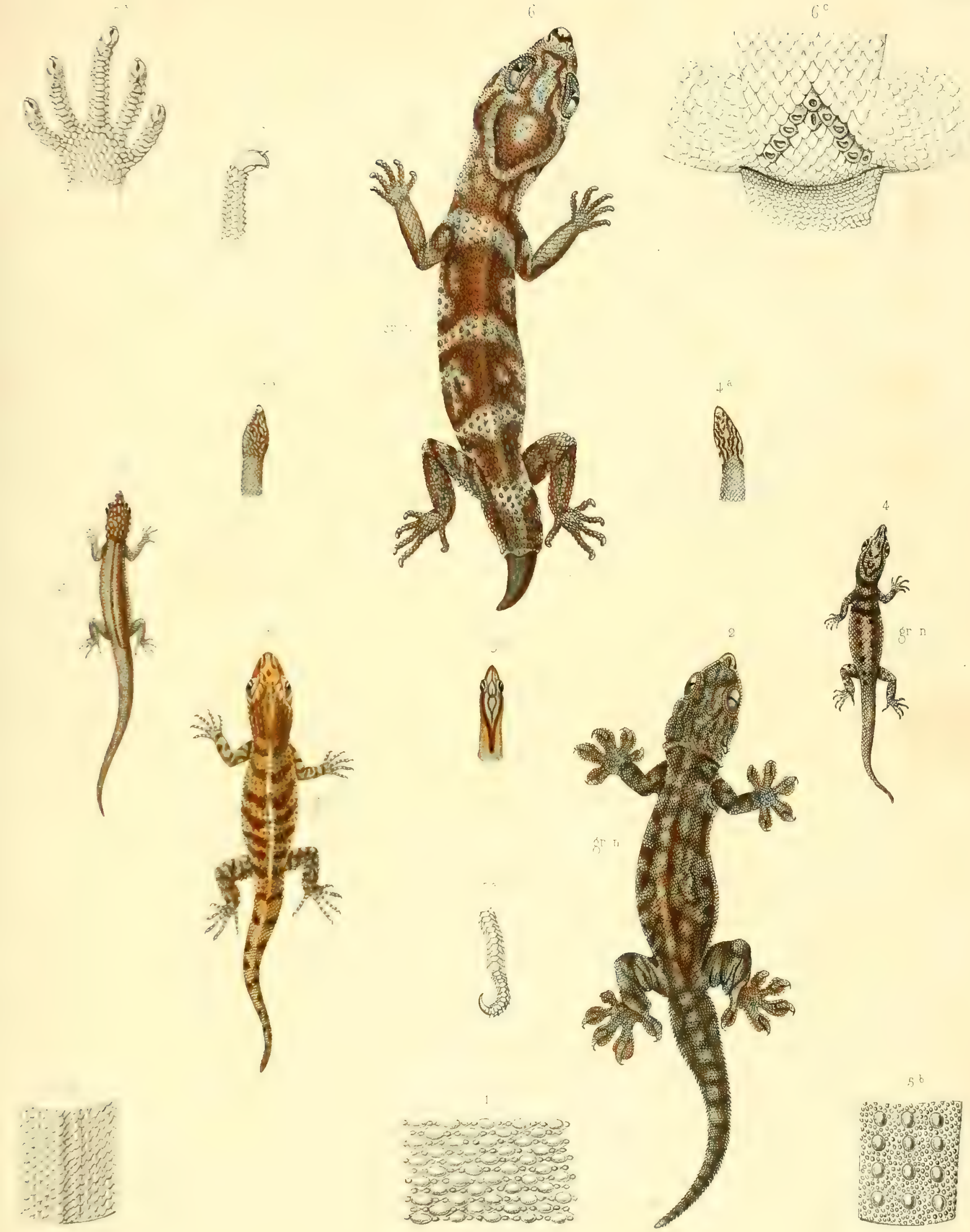

HÉMIDACTYLE TACHES-ROUSSES; 3. SPHÉRIODACTYLE BIZARRE 


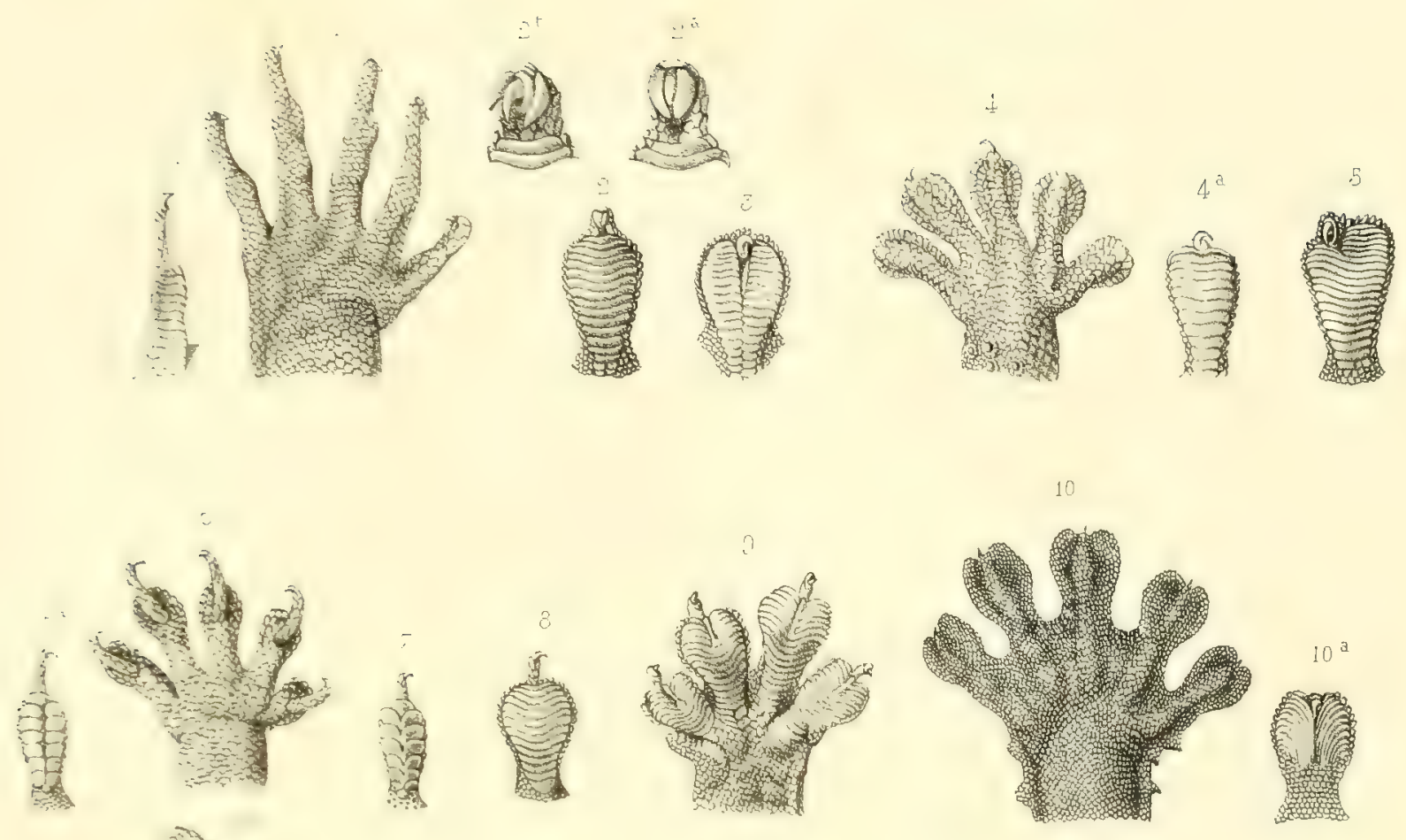

-
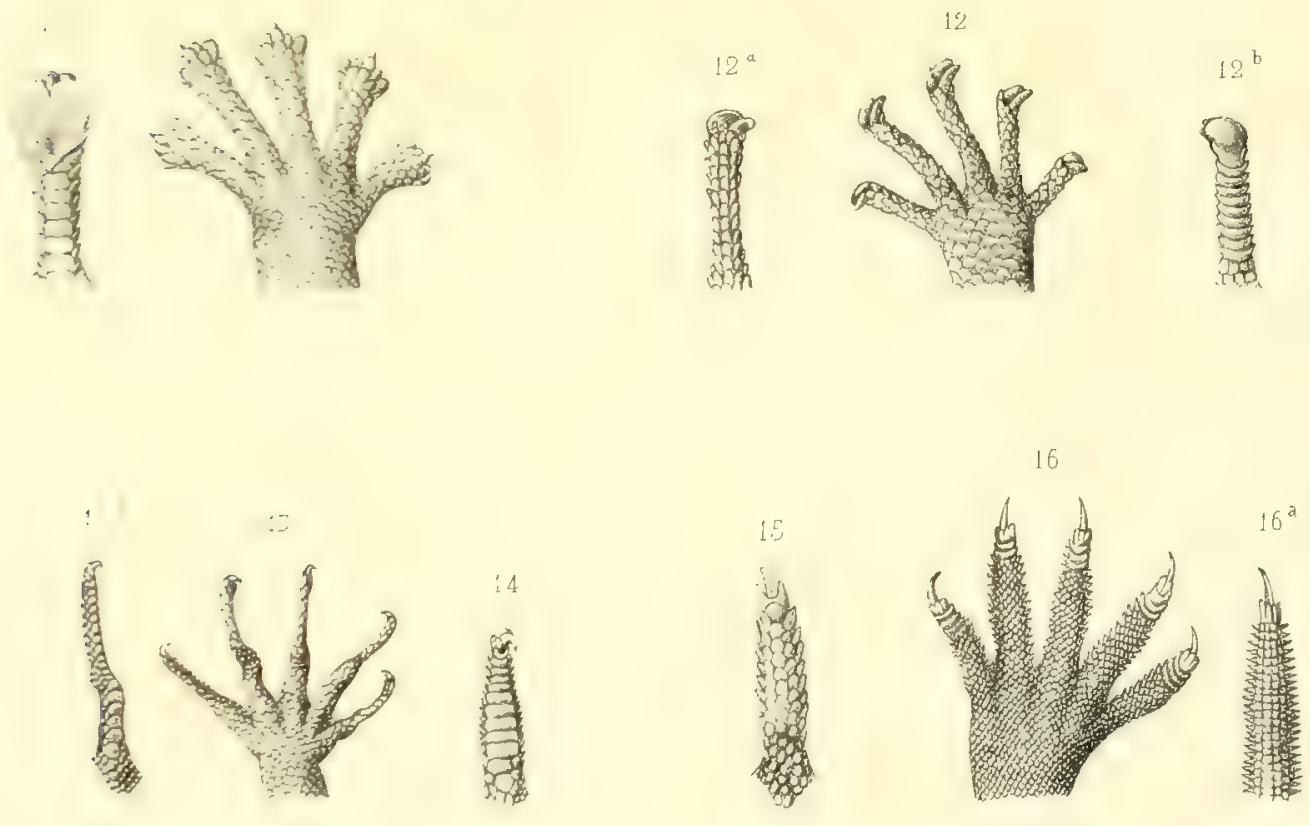

ous

GTS DE GECKOTIENS ( 2 et 3 fois la grand. nat.)

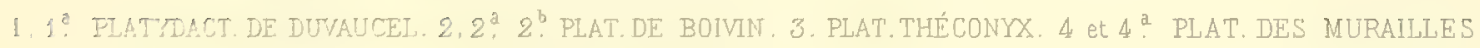

5. FLAT. DES SETCHEIJES. 6, 6, 6? HÉMIDACT. MABOUIA. 7. HÉM. TACHETÉ 8. HÉM. OUALIEN. 9. HÉM. ATELLE.

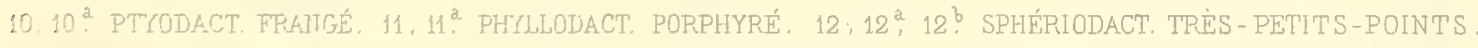

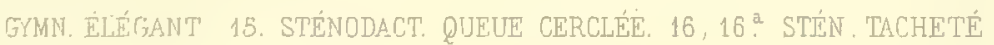





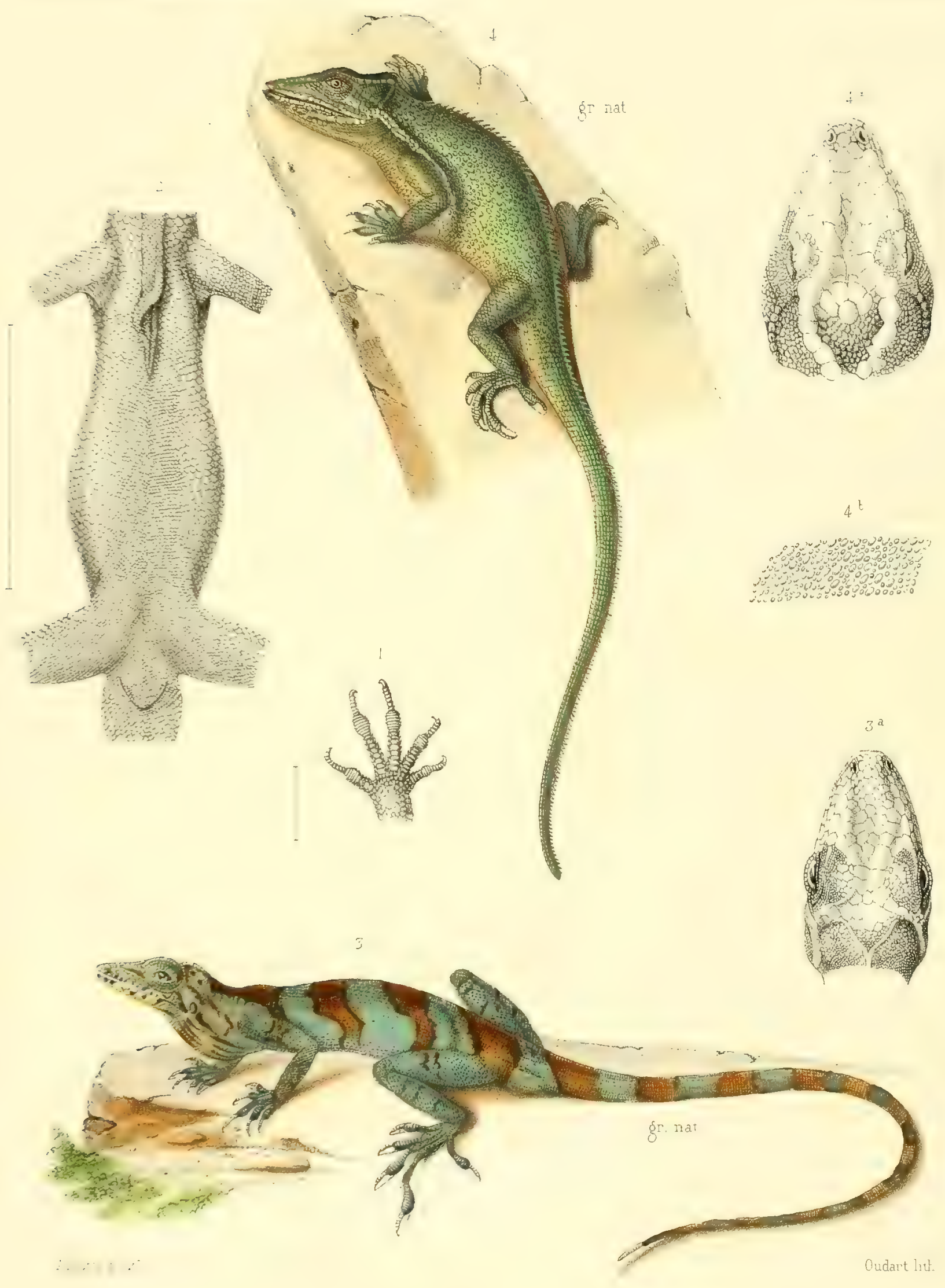





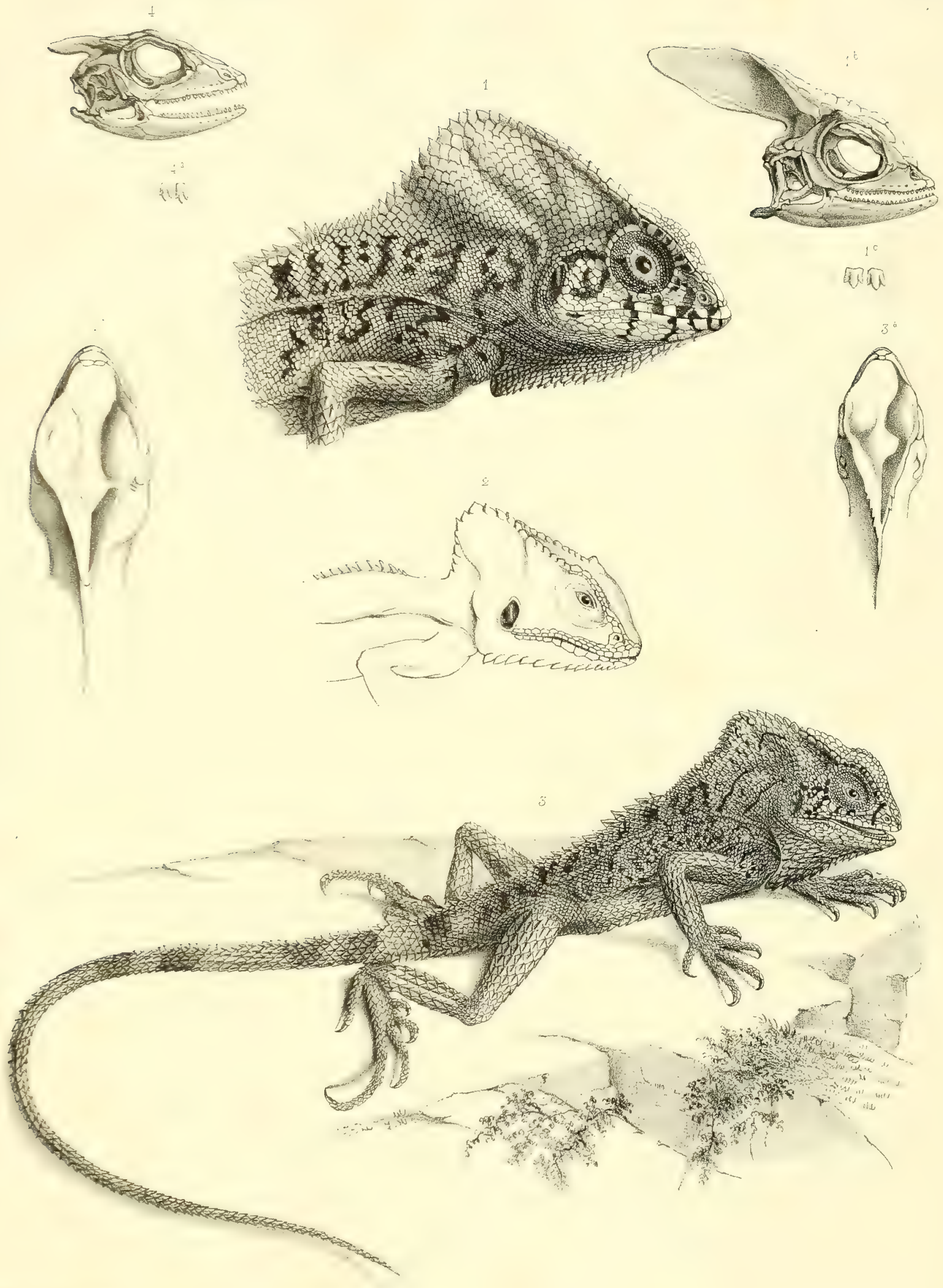





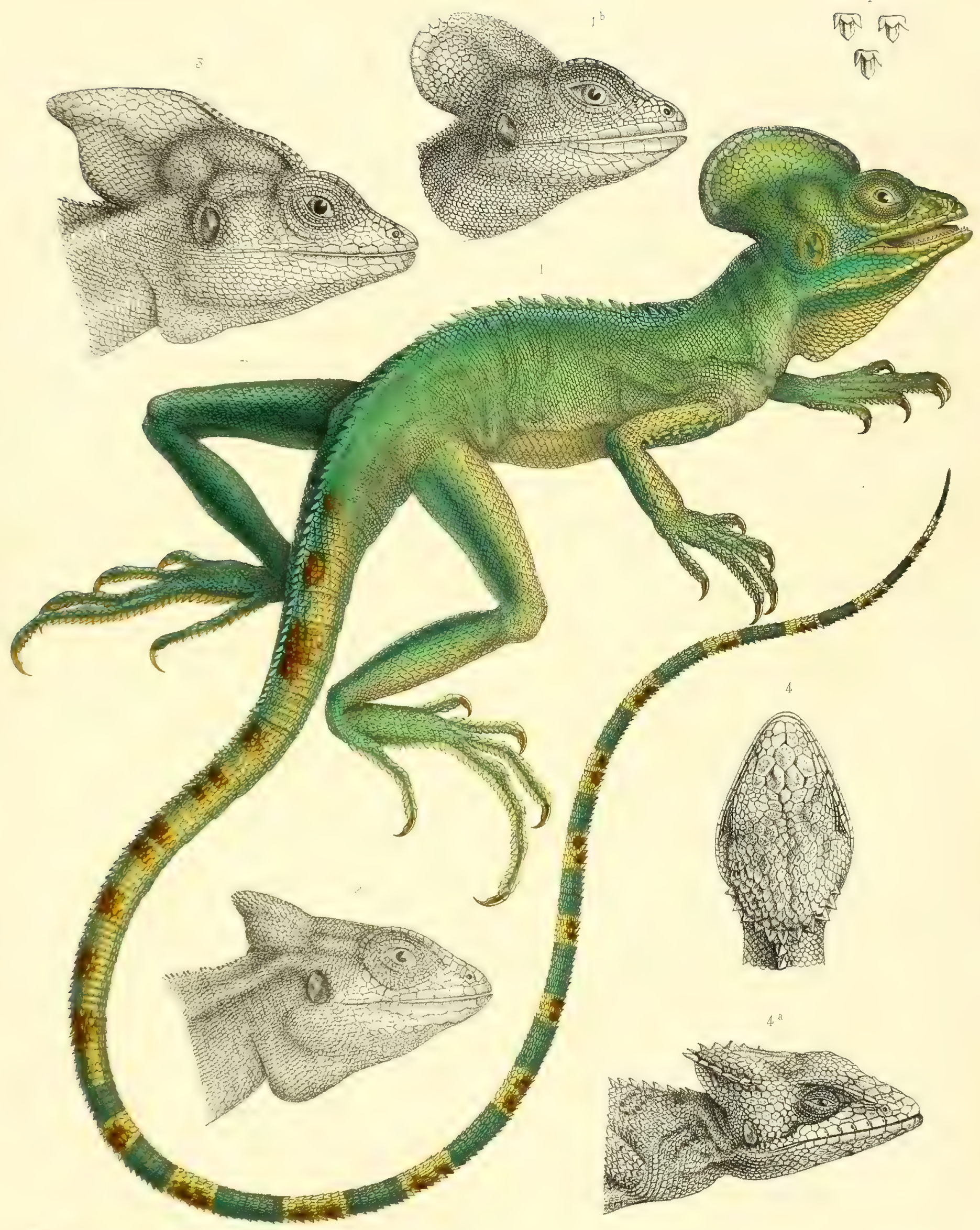



1
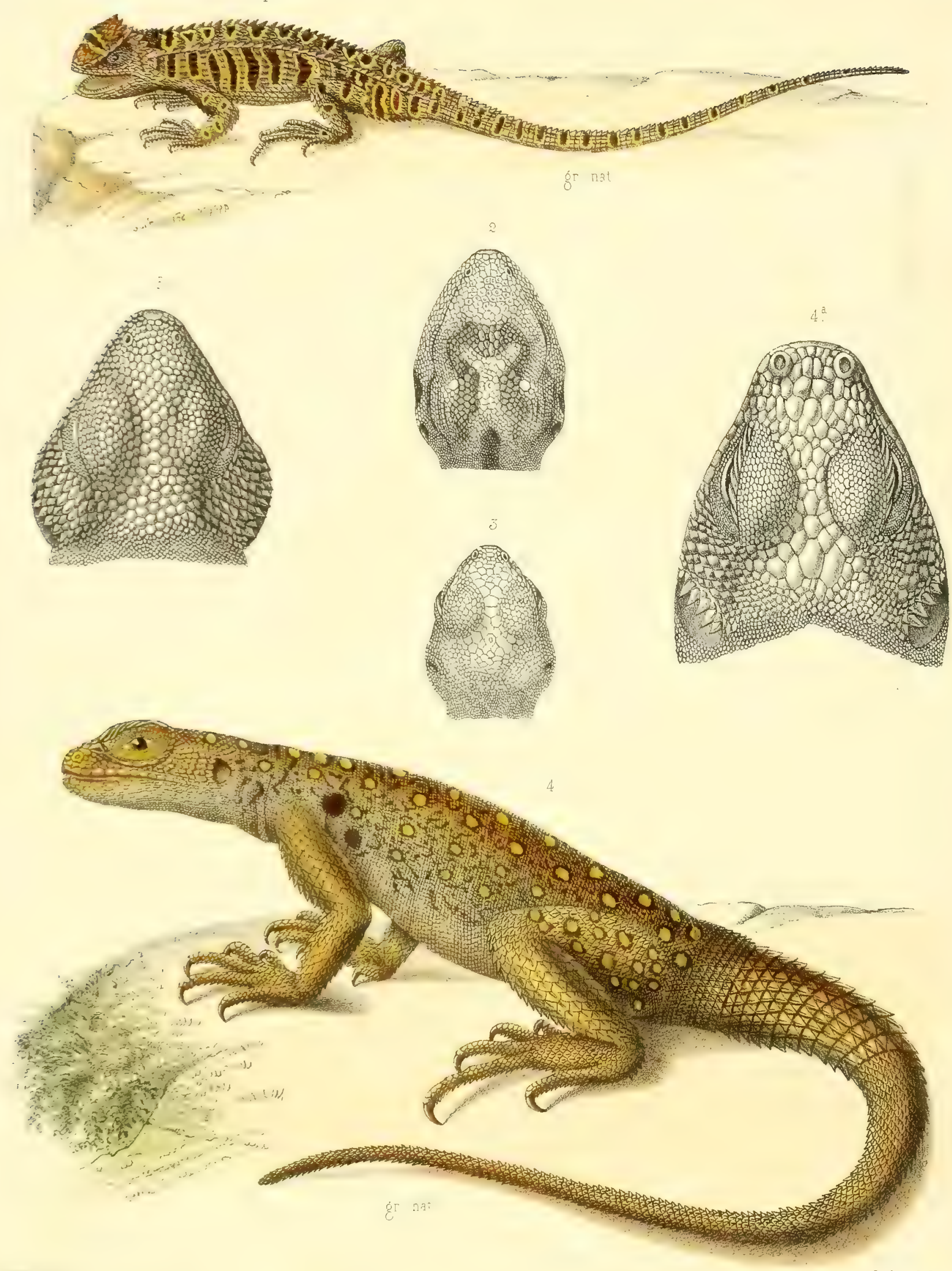

Oudart lith 


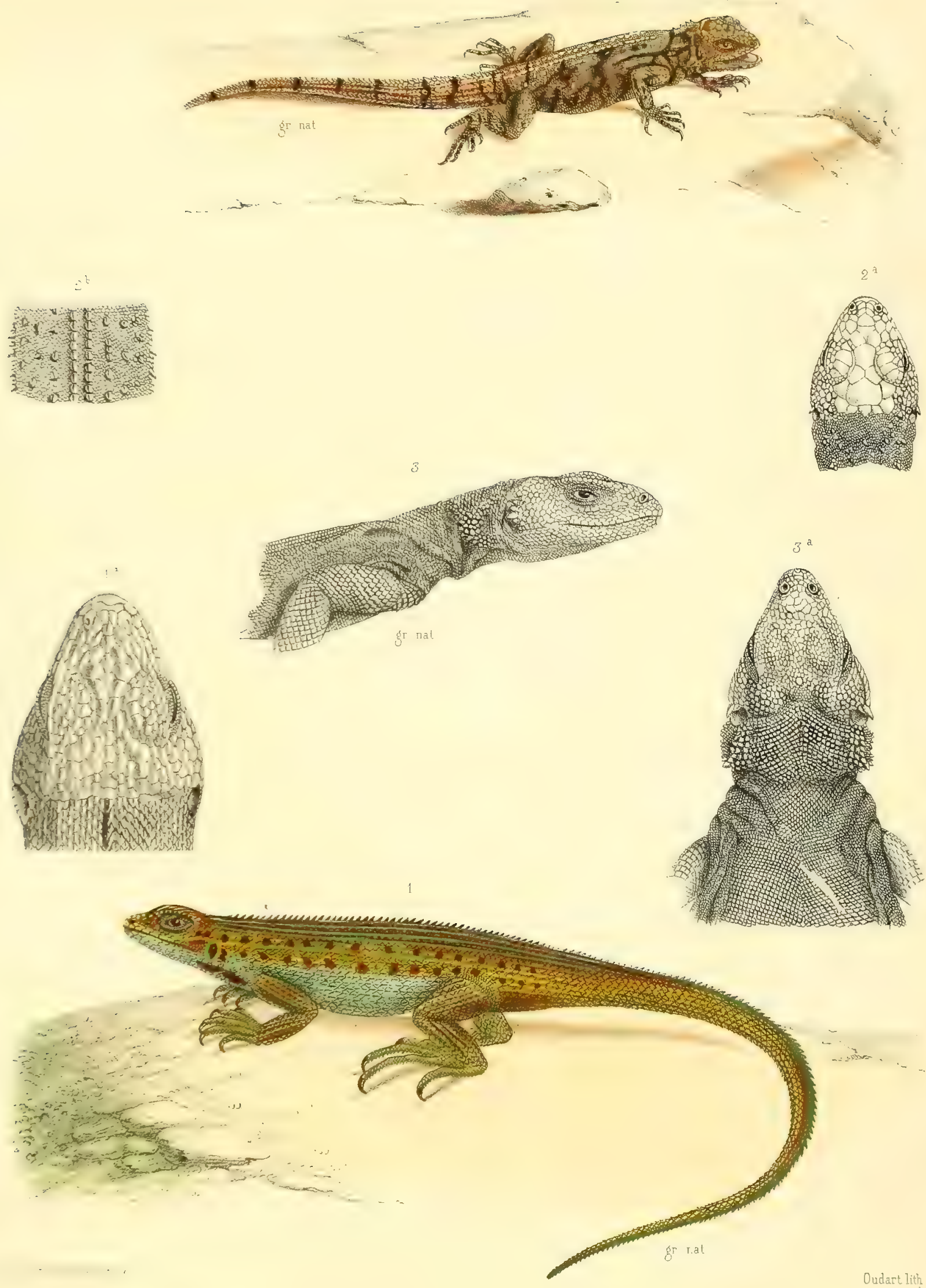


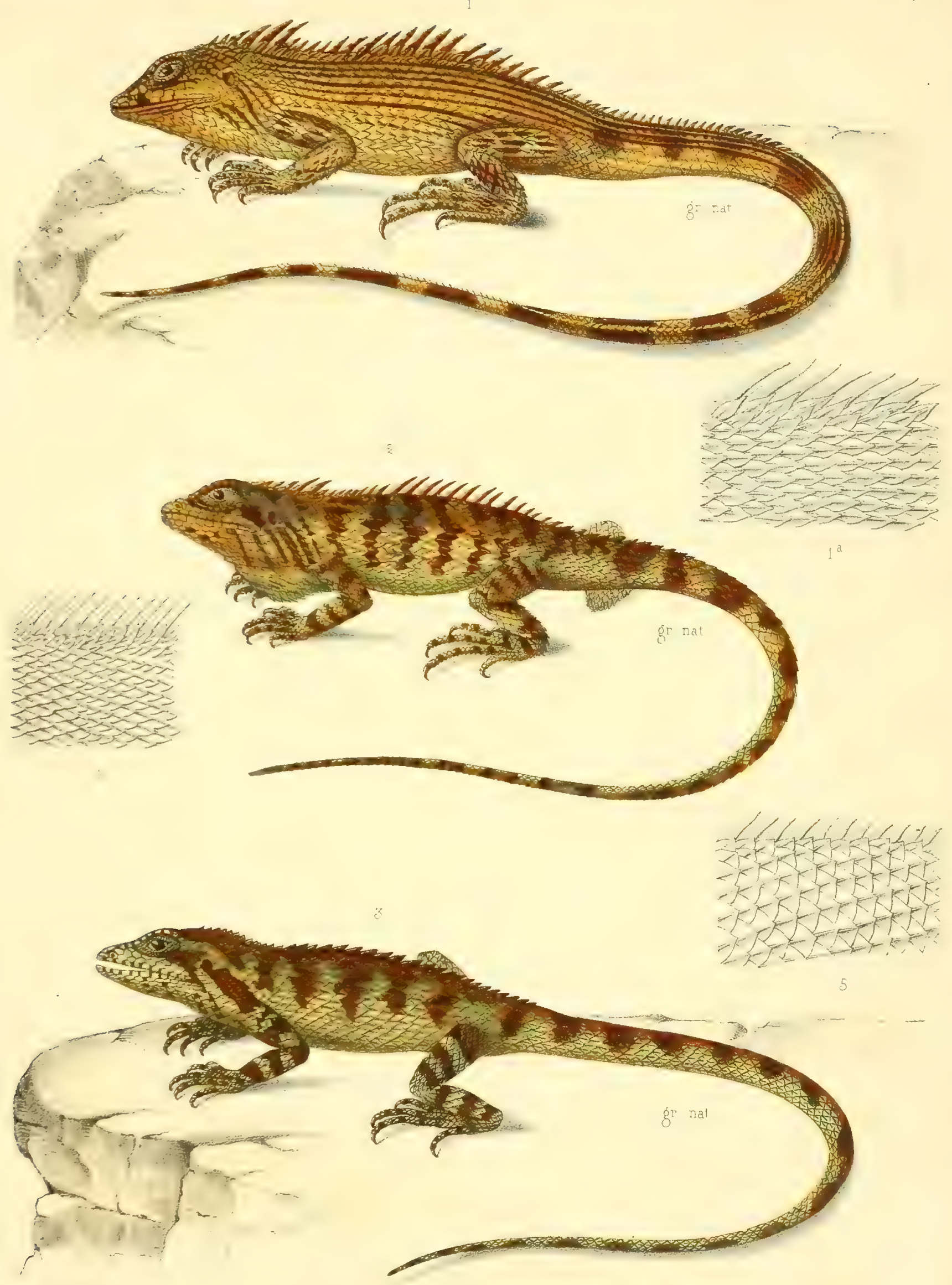

Oudart lith 






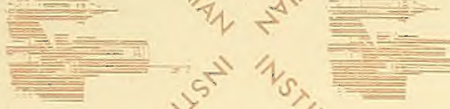

4400,140

(7.8)

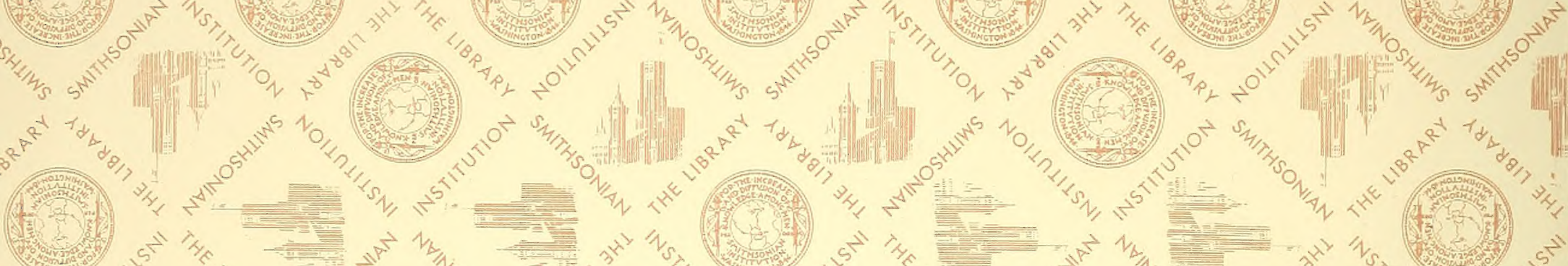

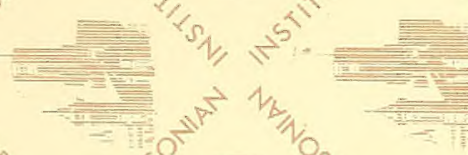

$(69)=$

$\infty$
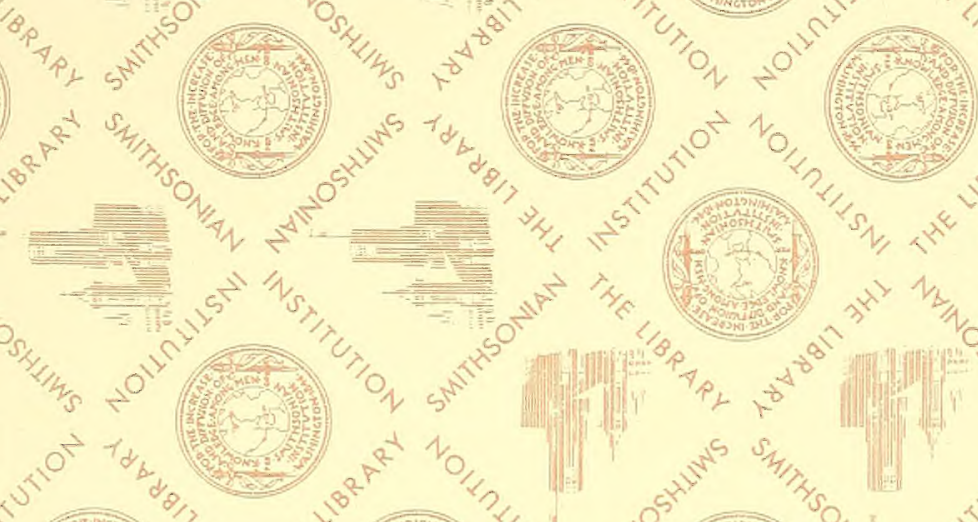

(1)

$={ }^{2} x^{2}=$

(4)

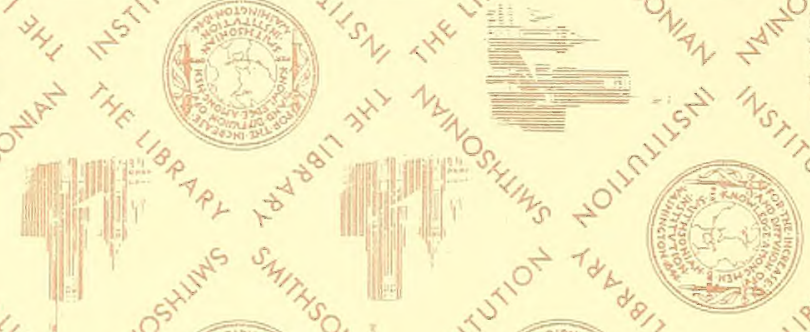

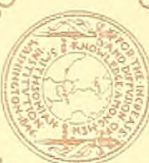

Ev

(2)
the

(c)

(2)

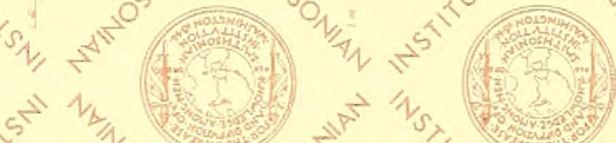

(I)

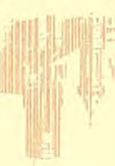

(1)
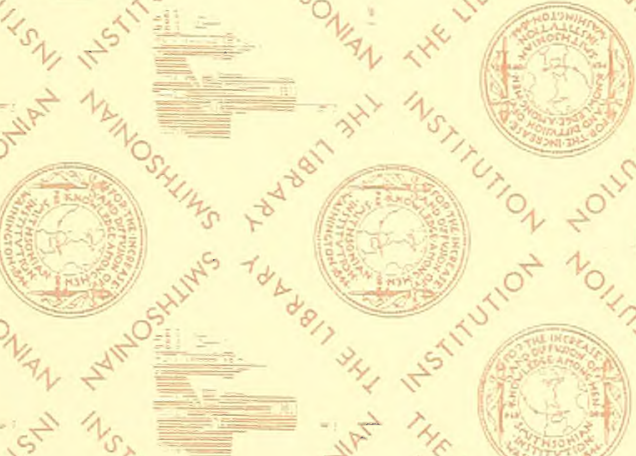

(
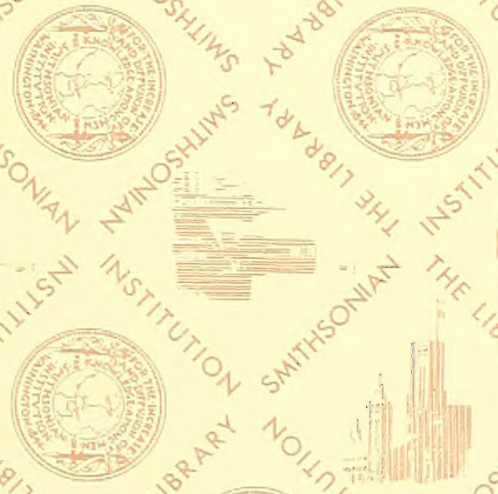

ply
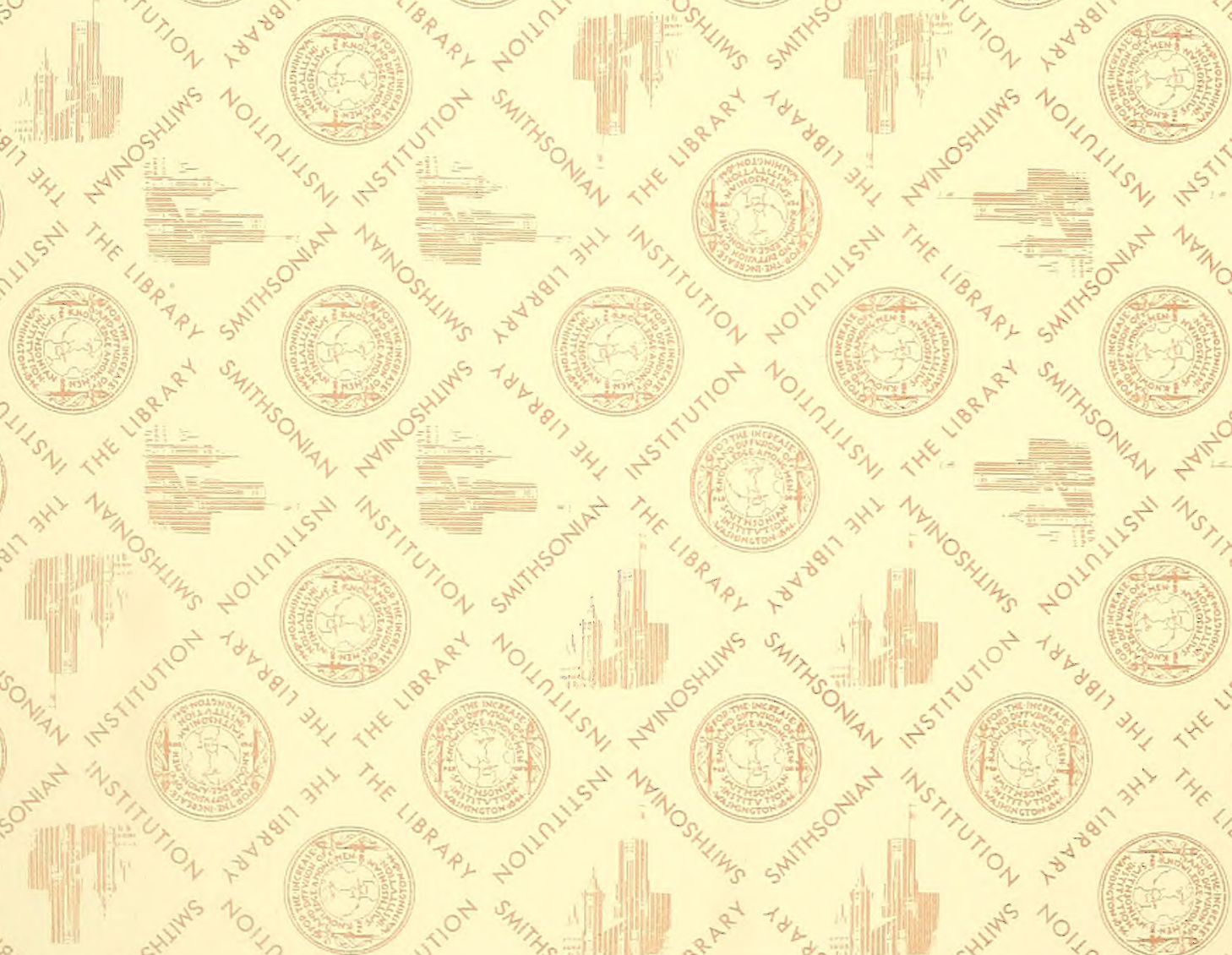

(3)

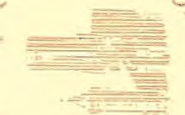

Hid

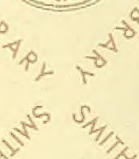



